\title{
Quarter-BPS states, multi-symmetric functions and set partitions
}

\author{
Christopher Lewis-Brown ${ }^{a}$ and Sanjaye Ramgoolam ${ }^{a, b}$ \\ ${ }^{a}$ School of Physics and Astronomy, Centre for Research in String Theory, \\ Queen Mary University of London, \\ London E1 4NS, United Kingdom \\ ${ }^{b}$ School of Physics and Mandelstam Institute for Theoretical Physics, \\ University of Witwatersrand, \\ Wits, 2050, South Africa \\ E-mail: c.h.lewis-brown@qmul.ac.uk, s.ramgoolam@qmul.ac.uk
}

ABSTRACT: We give a construction of general holomorphic quarter BPS operators in $\mathcal{N}=4$ SYM at weak coupling with $\mathrm{U}(N)$ gauge group at finite $N$. The construction employs the Möbius inversion formula for set partitions, applied to multi-symmetric functions, alongside computations in the group algebras of symmetric groups. We present a computational algorithm which produces an orthogonal basis for the physical inner product on the space of holomorphic operators. The basis is labelled by a $\mathrm{U}(2)$ Young diagram, a $\mathrm{U}(N)$ Young diagram and an additional plethystic multiplicity label. We describe precision counting results of quarter BPS states which are expected to be reproducible from dual computations with giant gravitons in the bulk, including a symmetry relating sphere and AdS giants within the quarter BPS sector. In the case $n \leq N$ ( $n$ being the dimension of the composite operator) the construction is analytic, using multi-symmetric functions and $\mathrm{U}(2)$ ClebschGordan coefficients. Counting and correlators of the BPS operators can be encoded in a two-dimensional topological field theory based on permutation algebras and equipped with appropriate defects.

KeYwords: $1 / N$ Expansion, AdS-CFT Correspondence

ARXIV EPRINT: 2007.01734 


\section{Contents}

1 Introduction 1

2 Review of zero coupling: symmetric functions, half-BPS operators and the quarter-BPS covariant basis

2.1 Half-BPS operators and symmetric functions 4

2.1.1 Monomial basis 5

2.1.2 Multi-trace basis 6

2.1.3 Schur basis 6

2.1.4 Correlators 8

$\begin{array}{lll}2.2 & \text { Quarter-BPS covariant operators } & 11\end{array}$

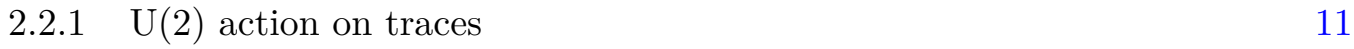

$\begin{array}{lll}2.2 .2 & \mathrm{U}(2) \text { representations } & 12\end{array}$

$\begin{array}{lll}2.2 .3 & \text { Operator definition } & 13\end{array}$

$\begin{array}{lll}2.2 .4 \text { Correlators } & 15\end{array}$

3 Quarter-BPS at weak coupling: key concepts $\quad \mathbf{1 5}$

$\begin{array}{lll}3.1 & \text { Background on construction of quarter BPS operators } & 16\end{array}$

$\begin{array}{lll}3.2 & \text { Steps in the construction of an SEP-compatible orthogonal BPS basis } & 17\end{array}$

4 Finite $\mathrm{N}$ combinatorics from many-boson states: multi-symmetric func$\begin{array}{ll}\text { tions and set partitions } & \mathbf{2 3}\end{array}$

4.1 Multi-symmetric functions as wavefunctions of a harmonic oscillator 23

4.2 Monomial and trace bases for multi-symmetric functions 25

4.3 Basis change for multi-symmetric functions 27

4.4 Möbius function for the poset of set partitions and combinatoric interpretation 30

4.5 More general $C$ and $\widetilde{C}$ matrices for $M$-matrix systems 33

4.6 Relation to other combinatorial quantities 36

5 Counting: $\mathrm{U}(2) \times \mathrm{U}(N)$ Young diagram labels and multiplicities at weak coupling

$5.1 \Lambda, p$ multiplicities and plethysms of $\mathrm{SU}(2)$ characters 41

5.2 Covariant trace bases 43

$\begin{array}{lll}5.3 & \text { General multiplicity formula } & 45\end{array}$

5.4 Hermite reciprocity and $p$-orbits of fixed $\mathcal{M}_{\Lambda, p} \quad 49$

$\begin{array}{ll}5.5 & \text { Calculation of multiplicities for simplest orbits } \\ 5.6\end{array}$

$\begin{array}{ll}5.6 & \text { Partitions with one dominant row or column } \\ 5.7 & 55\end{array}$

$\begin{array}{lll}5.7 & \text { Identifying a multiplicity space basis } & 57\end{array}$

6 Construction of orthogonal $\mathrm{U}(2) \times \mathrm{U}(N)$ Young-diagram-labelled basis 58

$\begin{array}{ll}\text { 6.1 Orthogonalisation and SEP compatibility } & 59\end{array}$

6.2 An example: field content $(2,2) \quad 61$

6.2.1 $\Lambda=[4]$ and $[3,1]$ sectors 62 
6.2.2 $\Lambda=[2,2]$ sector $\quad 63$

6.3 Normalisation conventions for BPS operators 64

$\begin{array}{lll}6.4 & \mathcal{F} \text {-orthogonalisation } & 68\end{array}$

6.4.1 The $\Lambda=[2,2]$ example $\quad 69$

$\begin{array}{lll}6.5 & \text { A shorter algorithm } & 69\end{array}$

6.5.1 General properties of Gram-Schmidt orthogonalisation $\quad 70$

6.5.2 Back to SEP-compatible bases $\quad 70$

$\begin{array}{ll}\text { 6.6 Choice of SEP-compatible basis } & 72\end{array}$

$\begin{array}{lll}6.7 & \text { Physical norms of BPS operators } & 73\end{array}$

$\begin{array}{lll}\text { 6.7.1 Norms of operators with multiplicity } & 74\end{array}$

$\begin{array}{lll}\text { 6.8 } & \text { Longest } p \text { for a given } \Lambda \text { and explicit quarter-BPS operators } & 75\end{array}$

6.9 Orthogonalisation at $\Lambda=[n]$ and $[n-1,1] \quad 76$

$\begin{array}{lll}6.9 .1 & \Lambda=[n] & 77\end{array}$

$\begin{array}{lll}6.9 .2 \Lambda=[n-1,1] & 77\end{array}$

$\begin{array}{ll}6.10 & \text { Alternative algorithm } \\ & 78\end{array}$

7 Vector space geometry in $\mathbb{C}\left(S_{n}\right)$ : BPS states from projectors for the intersection of finite $N$ and symmetrisation constraints in symmetric group algebras

$\begin{array}{lll}7.1 & n \text {-matrix model from permutations } & 82\end{array}$

$\begin{array}{lll}7.1 .1 & \text { Finite } N \text { relations } & 83\end{array}$

$\begin{array}{llr}7.1 .2 & \text { Flavour projection } & 84\end{array}$

7.1.3 Symmetrised traces $\quad 85$

$\begin{array}{lll}7.2 & \text { Multi-symmetric function isomorphism for } n \text { matrices } & 89\end{array}$

$\begin{array}{lll}7.3 & \text { Finite } N \text { symmetrisation operator on } \mathbb{C}\left(S_{n}\right) & 92\end{array}$

8 Hidden 2D topology: permutation TFT2 for the counting and correlators $\begin{array}{ll}\text { at weak coupling } & 97\end{array}$

9 Summary and outlook $\quad 99$

$\begin{array}{ll}\text { A } \Lambda=[3,2] \text { sector } & 101\end{array}$

$\begin{array}{lll}\text { A.1 BPS operators } & 102\end{array}$

$\begin{array}{lll}\text { A.2 Norms of BPS operators } & 103\end{array}$

B $\Lambda=[4,2]$ sector $\quad 103$

$\begin{array}{lll}\text { B.1 Free field covariant basis from traces } & 104\end{array}$

$\begin{array}{lll}\text { B.2 Quarter-BPS basis } & 105\end{array}$

$\begin{array}{lll}\text { B.3 Norms of operators with multiplicity } & 113\end{array}$

$\begin{array}{lll}\text { B.4 Norms of BPS operators } & 115\end{array}$

$\begin{array}{ll}\mathrm{C} \quad \Lambda=[3,3] \text { sector } & 117\end{array}$ 


\section{Introduction}

Half-BPS operators in $\mathcal{N}=4 \mathrm{SYM}$ with $\mathrm{U}(N)$ gauge group are related, via the AdS/CFT correspondence [1-3] to extremely rich physics including Kaluza-Klein gravitons, giant gravitons [4-6] and LLM geometries [7]. These operators are constructed from gauge invariant holomorphic functions of a single complex matrix $X=\phi_{1}+i \phi_{2}$, where $\phi_{1}, \phi_{2}$ are two of the six hermitian scalars in the adjoint of the gauge group. The two-point function of a holomorphic with an anti-holomorphic operator gives an inner product on the space of BPS operators. An orthogonal basis of operators labelled by Young diagrams $R$, with length no greater than $N$ was constructed in [9]. The Young diagram $R$ is identified by the Casimirs of a $\mathrm{U}(N)$ symmetry in the theory. Three-point functions were computed in terms of Littlewood-Richardson coefficients [9]. This allowed a general identification of gauge invariant operators dual to giant gravitons in the AdS spacetime, extending the results in [8]. An important guide to the precise identification of giant graviton geometries dual to particular Young diagrams, was the stringy exclusion principle (SEP), which refers to the fact that certain states disappear from the Hilbert space of the theory as $N$ changes to $N-1$ [10]. States corresponding to the Young diagrams with $N$ rows are precisely all the states which disappear from the Hilbert space as the rank of the gauge group changes from $N$ to $N-1$. Recent computations of correlators of giant gravitons in the AdS space-time have demonstrated agreement between CFT and AdS [11-14]. Despite significant progress on the quarter-BPS and eighth-BPS sectors, both in the construction of CFT operators and in the construction of quantum states in the dual space-time, a comparable level of understanding of AdS/CFT in this sector remains an important challenge.

From consideration of the quantization of moduli spaces of giant gravitons which are large in the $S^{5}$ directions on $A d S_{5} \times S^{5}$, it has been argued that the quantum states arising are those of an $N$-boson system in a harmonic oscillator [15-17]. From consideration of moduli spaces of giants which are large in the $A d S_{5}$ directions, the same Hilbert space has been arrived at [18]. Yet another perspective comes from the strong coupling matrix dynamics leading to commuting $N \times N$ matrices [19]. The counting of quarter and eighth BPS states has been derived by calculating the quarter and eighth-BPS cohomology [20].

The construction of quarter BPS operators from the gauge theory side has been developed in [21-24]. At zero field theory coupling, the quarter BPS states are general holomorphic operators built from two complex matrices $X$ and $Y$. A subspace of these operators is annihilated by the one-loop dilatation operator and forms the weak coupling quarter BPS space. An important outcome of these papers is that the weak coupling quarter BPS operators form the orthogonal subspace, in the free field inner product, of the operators which contain commutators $[X, Y]$ within a trace. This a well-defined characterisation of the quarter-BPS operators at finite $N$.

An orthogonal basis for the free field inner product, in terms of Young diagrams, was found in $[25,26]$, which was covariant under the $\mathrm{U}(2)$ symmetry of the quarter BPS sector or $\mathrm{U}(3)$ for eighth-BPS, and valid at finite $N$. Closely related orthogonal bases were obtained in [27-29] and the relation between the different bases in terms of free field conserved charges was elucidated in [30]. In the free-field $\mathrm{U}(2)$ covariant constructions of 
quarter BPS operators [25, 26] having a total of $n$ copies of $X$ and $Y$, the labels consist of a Young diagram $R$ with $n$ boxes and columns no longer than $N$, a Young diagram $\Lambda$ with $n$ boxes and columns no longer than 2, along with a label $\tau$ which runs over the multiplicity of trivial $S_{n}$ irreps in $R \otimes R \otimes \Lambda$. The Young diagram label $\Lambda$ is also a representation of the global symmetry $U(2)$. The construction of weak-coupling quarter-BPS operators based on this new understanding of the finite $N$ inner product was further developed in $[31,32]$. The finite $N$ construction of quarter BPS operators was given in terms of a projector $\mathcal{P}_{N}$ in $\mathbb{C}\left(S_{n}\right)$, which projects to the intersection of two subspaces of $\mathbb{C}\left(S_{n}\right)$ [32]. One subspace is associated with the symmetrised traces at large $N$, another with the finite $N$ cutoff on the free field basis.

In [33], the eighth-BPS Hilbert space of $N$ bosons in a 3D harmonic oscillator obtained from quantizing the space of holomorphic polynomials in $\mathbb{C}^{3}$ was used to give a labelling of eighth-BPS states by a $\mathrm{U}(3)$ Young diagram, along with a $\mathrm{U}(N)$ Young diagram and an additional multiplicity label constructed from the pair of Young diagrams. We will refer to this multiplicity label as a plethystic multiplicity label, due to the role of plethysm operations in its definition. By restricting to the quarter BPS states there is a labelling using a $\mathrm{U}(2)$ Young diagram, a $\mathrm{U}(N)$ Young diagram and a plethystic multiplicity label. In another development, a special class of quarter BPS operators at weak coupling was found to be related to Brauer algebra constructions [34].

There has not been so far, a general construction of quarter-BPS operators at weak coupling and finite $N$, which includes a $\mathrm{U}(2)$ Young diagram label alongside a $\mathrm{U}(N)$ Young diagram label. In this paper, we will address this open problem and give a basis of operators which are quarter-BPS at weak coupling, orthogonal with respect to the free field inner product, and labelled by a $\mathrm{U}(2)$ Young diagram, a $\mathrm{U}(N)$ Young diagram, alongside an associated multiplicity label depending on these two Young diagrams. The virtue of having a $\mathrm{U}(N)$ Young diagram is that the disappearance of states upon a reduction of $N$ to $N-1$ can be directly expressed in terms of this Young diagram - the disappearing states as $N$ is reduced to $N-1$ are precisely the ones corresponding to Young duagrams with exactly $N$ rows. We may therefore describe our basis as an SEP-compatible (SEP = stringy exclusion principle) basis which is also $\mathrm{U}(2)$ covariant.

The key ingredient which allows us to find a manifestly SEP-compatible U(2) covariant construction of quarter BPS states is the mathematics of multi-symmetric functions [35-37]. When gauge invariant functions of two matrices $X, Y$ are evaluated on diagonal matrices $X=\operatorname{Diag}\left(x_{1}, x_{2}, \cdots, x_{N}\right)$ and $Y=\operatorname{Diag}\left(y_{1}, y_{2}, \cdots, y_{N}\right)$, we get polynomials which are invariant under the $\sigma \in S_{N}$ acting simultaneously as

$$
\begin{aligned}
x_{i} & \rightarrow x_{\sigma(i)} \\
y_{i} & \rightarrow y_{\sigma(i)}
\end{aligned}
$$

These polynomials are called multisymmetric functions. More generally, we can have variables $x_{i}^{a}$ with $a \in\{1,2, \cdots, M\}$ and $i \in\{1,2, \cdots, N\}$. Polynomials invariant under simultaneous $S_{N}$ permutations of all the $M$ vectors are more general multi-symmetric functions. There is a rich mathematics associated with changing between different bases 
of multi-symmetric functions for any $M$ which is relevant in this paper and is controlled by an underlying structure of set partitions.

The paper is organised as follows. Section 2 reviews the connection between half BPS operators and symmetric functions before covering the construction of orthogonal bases of half and quarter BPS states at zero coupling. This is based on the use of permutation algebras and Schur-Weyl duality relations between permutation representation theory and the representation theory of $\mathrm{U}(N)$. Section 3 is an introduction to the key mathematical tools and physical concepts we will use in this paper. In section 4 use the combinatorics of set partitions to derive results on the transformation between two bases for multi-symmetric functions. The first is the trace basis. Elements of this basis set are obtained by specifying a trace structure for matrices $X, Y$, or more generally $X^{1}, \cdots, X^{M}$ and specialising to diagonal matrices. Another basis is the multi-symmetric monomial basis, which allows a simple description of finite $N$ cut-offs. In section 5 , we start from the observation that every vector partition $\mathbf{p}$ defines an associated partition $p$, which is invariant under the action of the $\mathrm{U}(2)$ transformations which interchange $X, Y$. We use results on plethysms of $\mathrm{SU}(2)$ representations to obtain detailed expressions for refined multiplicities depending on a pair of Young diagram $\Lambda, p$, where $\Lambda$ is a $\mathrm{U}(2)$ Young diagram and $p$ is a Young diagram constrained to have no more than $N$ rows, which we refer to as a $\mathrm{U}(N)$ Young diagram. In section 6 we describe an algorithm for producing a basis of operators labelled by the pair of Young diagrams $(\Lambda, p)$ alongside the appropriate multiplicity label. The basis is orthogonal under the free field inner product. In section 7 we elucidate the vector space geometry within $\mathbb{C}\left(S_{n}\right)$, involving the interplay between a projector for the $\mathrm{U}(2)$ flavour symmetry, a projector for the symmetrisation of traces $\mathcal{P}$ and an operator $\mathcal{F}_{N}$ whose kernel implements finite $N$ constraints. This discussion allows us to show that the counting and two-point correlators of quarter-BPS operators at weak coupling can be expressed in terms of observables in two-dimensional topological field theory based on permutation group algebras with appropriate defects.

In appendices $\mathrm{A}-\mathrm{C}$ we give examples of the quarter-BPS operators in the $\Lambda=$ $[3,2],[4,2]$ and $[3,3]$ sectors. These operators were generated using code we have made available on Github [74].

\section{Review of zero coupling: symmetric functions, half-BPS operators and the quarter-BPS covariant basis}

The half-BPS sector is composed of multi-traces of a single complex matrix $X$. We diagonalise $X$ in terms of its eigenvalues

$$
X=\left(\begin{array}{ccccc}
x_{1} & 0 & 0 & \ldots & 0 \\
0 & x_{2} & 0 & \ldots & 0 \\
0 & 0 & x_{3} & \ldots & 0 \\
\vdots & \vdots & \vdots & \ddots & \vdots \\
0 & 0 & 0 & \ldots & x_{N}
\end{array}\right)
$$


Thus any multi-trace of $X$ can instead be written as a function of the eigenvalues $x_{1}, x_{2}, \ldots, x_{N}$. These functions must be completely symmetric in the $N$ variables. The theory of such functions has long been studied in mathematics, and they have many interesting properties [38]. In this section we review the basic definition, three of the commonly used bases and give the connection to the half-BPS sector of $\mathcal{N}=4$ super Yang-Mills.

Since correlators of single matrix multi-traces are not renormalised as we go from zero coupling to weak coupling to strong coupling [39], these bases remain half-BPS in all regimes.

After covering the half-BPS sector, we then review the covariant basis [25, 26] for the free field quarter-BPS sector. This involves two complex matrices $X$ and $Y$, in general non-commuting, and therefore we cannot express generic multi-traces purely in terms of their eigenvalues as we did in the half-BPS sector.

For the quarter-BPS sector, there is a step-change as we transition from zero coupling to weak coupling. After turning on interactions, some linear combinations of multi-traces recombine into long non-BPS multiplets, and therefore the covariant basis introduced here is only a basis for the quarter-BPS sector in the free theory. The remainder of the paper focuses on finding a generalisation of the covariant basis for the quarter-BPS sector at weak coupling.

\subsection{Half-BPS operators and symmetric functions}

Symmetric functions are defined as polynomials in the $N$ variables $x_{1}, x_{2}, x_{3}, \ldots, x_{N}$ that are invariant under all permutations of the $x_{i}$. More explicitly, given a polynomial $f\left(x_{1}, x_{2}, \ldots, x_{N}\right), f$ is a symmetric function if

$$
f\left(x_{1}, x_{2}, \ldots, x_{N}\right)=f\left(x_{\sigma(1)}, x_{\sigma(2)}, \ldots, x_{\sigma(N)}\right)
$$

for all $\sigma \in S_{N}$, the group of permutations of $\{1,2, \ldots, N\}$.

We can take the infinite $N$ limit of this definition by defining symmetric functions as formal power series in infinitely many variables $x_{1}, x_{2}, \ldots$ To return to the finite $N$ case (or to reduce a symmetric function in $M>N$ variables to one in $N$ variables), we can set $x_{N+1}=0, x_{N+2}=0, \ldots$

Physically, symmetric polynomials in $N$ variables are the wavefunctions associated to $N$ bosons moving in one dimension. The variable $x_{i}$ corresponds to the position of the $i$ th boson, and the symmetric nature of the function is exactly the condition on the wavefunction that the state must be symmetric between identical bosons.

There are several different bases for the ring of symmetric functions, of which we will look at three. In each of these bases, each basis element consists of polynomials of a single degree, $n$, and the basis for the degree $n$ subspace is labelled by the partitions of $n$, which we now describe.

A partition $p$ of $n$ is a set of weakly decreasing positive integers $\lambda_{1} \geq \lambda_{2} \geq \cdots \geq \lambda_{k}$ which sum to $n$. We call $\lambda_{j}$ the components of $p, k=l(p)$ the length of $p$ and write $p=\left[\lambda_{1}, \lambda_{2}, \ldots, \lambda_{k}\right]$. We denote partitions of $n$ by $p \vdash n$. 
An alternative way of defining a partition $p$ is to give the number, $p_{1}$, of $\lambda_{j}$ equal to 1 , the number, $p_{2}$, of $\lambda_{j}$ equal to 2 , etc. We write

$$
p=<p_{1}, p_{2}, \cdots>
$$

We call the $p_{i}$ the multiplicities of $p$, and this is the multiplicity notation. The $p_{i}$ satisfy

$$
\sum_{i} i p_{i}=n
$$

A partition $p \vdash n$ can be thought of visually using a Young diagram. This consists of a set of boxes arranged in rows. The first row has length $\lambda_{1}$, the second $\lambda_{2}$, etc. For example for $p=[4,2,1]$ the associated Young diagram is

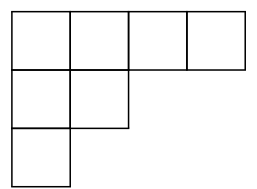

We will in general use the same notation for $p$ and the associated Young diagram. When it is necessary to make a distinction, we will use $Y(p)$ for the Young diagram.

For a partition $p=\left[\lambda_{1}, \lambda_{2}, \ldots\right]$, the conjugate partitions is defined by $p^{c}=\left[\mu_{1}, \mu_{2}, \ldots\right]$ with $\mu_{i}$ defined so that

$$
\begin{array}{ll}
\lambda_{j} \geq i & \text { if } j \leq \mu_{i} \\
\lambda_{j}<i & \text { if } j>\mu_{i}
\end{array}
$$

Intuitively, we transpose the Young diagram of $p$, so that rows of $p$ become columns of $p^{c}$ and vice versa.

\subsubsection{Monomial basis}

We start with the monomial basis. Given a partition $p=\left[\lambda_{1}, \lambda_{2}, \ldots, \lambda_{k}\right]$ of $n$, take the monomial

$$
x_{1}^{\lambda_{1}} x_{2}^{\lambda_{2}} \ldots x_{k}^{\lambda_{k}}
$$

and then add all distinct permutations of it to form a symmetric function. So for example if we take $p=[3,1,1]$ (and use $N=3$ for simplicity), the associated monomial basis element is

$$
m_{[3,1,1]}=x_{1}^{3} x_{2} x_{3}+x_{1} x_{2}^{3} x_{3}+x_{1} x_{2} x_{3}^{3}
$$

For $p=<p_{1}, p_{2}, \cdots>$, we can define the monomial functions more formally by

$$
m_{p}=\left(\prod_{i} \frac{1}{p_{i} !}\right) \sum_{\sigma \in S_{N}} x_{\sigma(1)}^{\lambda_{1}} x_{\sigma(2)}^{\lambda_{2}} \ldots x_{\sigma(l(p))}^{\lambda_{l(p)}}
$$

where $N \geq l(p)$ and the normalisation in front accounts for non-trivial coefficients introduced by redundancies in the components of $p$. 
For future convenience, we also define the rescaled monomial function $M_{p}$ to be (2.9) without the normalisation factor.

$$
M_{p}=\sum_{\sigma \in S_{N}} x_{\sigma(1)}^{\lambda_{1}} x_{\sigma(2)}^{\lambda_{2}} \ldots x_{\sigma(l(p))}^{\lambda_{l(p)}}
$$

Lowering $N$ reduces the degrees of freedom in the system, and therefore we expect the basis to change to account for this. For the monomial basis, this process is particularly simple. For $N \geq n$, which we refer to as large $N$, (2.10) forms a basis of the degree $n$ symmetric functions. For $N<n$, we set $x_{N+1}=x_{N+2}=\cdots=0$, and therefore those functions labelled by $p$ with $l(p)>N$ all vanish identically, while those with $l(p) \leq N$ form a basis for the remaining space.

\subsubsection{Multi-trace basis}

The second basis we consider is the multi-trace basis, normally called the power-sum basis in the mathematics literature. This is constructed from polynomials of the form

$$
T_{k}=\sum_{i=1}^{N} x_{i}^{k}
$$

For a partition $p=\left[\lambda_{1}, \lambda_{2}, \ldots \lambda_{k}\right]$, the symmetric function is

$$
T_{p}=\prod_{i=1}^{k} T_{\lambda_{i}}
$$

Consider a $N \times N$ diagonal matrix $X$ with entries $x_{i}$, as in (2.1). Then (2.11) can be written in terms of $X$ as $T_{k}=\operatorname{Tr} X^{k}$. For the general symmetric function (2.12) we have

$$
T_{p}=\prod_{i}\left(\operatorname{Tr} X^{i}\right)^{p_{i}}
$$

This is the link between symmetric functions and the multi-trace basis for the half-BPS sector of $\mathcal{N}=4$ super Yang-Mills.

When we reduce from large $N$ to $N<n$, non-trivial relationships are induced among the multi-traces of a given degree, which can be understood from the Cayley-Hamilton theorem. As we saw for the monomials, one can take those multi-traces labelled by $p$ with $l(p) \leq N$, and they will form a basis. However, in this case those multi-traces associated to $p$ with $l(p)>N$ do not vanish, instead they are complicated linear sums of the remaining basis elements.

\subsubsection{Schur basis}

Finally, we look at the Schur basis. These are labelled by partitions $R \vdash n$, thought of as representations of the symmetric group $S_{n}$.

$$
s_{R}=\sum_{p \vdash n} \frac{1}{z_{p}} \chi_{R}(p) T_{p}
$$


where $z_{p}$ is the order of the centralizer of a permutation in $S_{n}$ with cycle type $p=<$ $p_{1}, p_{2}, \cdots>$, and is given explicitly by

$$
z_{p}=\prod_{i} i^{p_{i}} p_{i} !
$$

Since the Schur and monomial functions form a basis for the degree $n$ symmetric functions, there is a basis change matrix transforming between them. This is given by the Kostka numbers $K_{R p}$

$$
s_{R}=\sum_{p \vdash n} K_{R p} m_{p}
$$

The Kostka have a combinatoric interpretation in terms of the number of semi-standard Young tableaux of shape $R$ and evaluation $p$. These Young tableaux are defined in section 2.2.2.

Let $V$ be the $N$-dimensional space on which the Yang-Mills scalar field $X$ acts, and consider $V^{\otimes n}$, the $n$-times tensor product of $V$. The permutation group $S_{n}$ acts on $V^{\otimes n}$ by permuting the factors, and we also have the operator $X^{\otimes n}$, which applies $X$ to each tensor factor. Take the trace over $V^{\otimes n}$ of a product between $\sigma \in S_{n}$, a permutation of cycle type $p \vdash n$, and $X^{\otimes n}$. This is

$$
\operatorname{Tr}\left(\sigma X^{\otimes n}\right)=\prod_{i}\left(\operatorname{Tr} X^{i}\right)^{p_{i}}=T_{p}
$$

Using this, we can re-write (2.14) as

$$
s_{R}=\mathcal{O}_{R}=\frac{1}{n !} \sum_{\sigma \in S_{n}} \chi_{R}(\sigma) \operatorname{Tr}\left(\sigma X^{\otimes n}\right)
$$

This is the standard definition of the Schur operators in the half-BPS sector first introduced in $[9]$.

The Schur basis behaves in the same way as monomial basis for $N<n$. Those basis elements with $l(R)>N$ vanish identically, while those with $l(R) \leq N$ form the basis for the reduced space. In the context of the $\mathcal{N}=4 \mathrm{SYM}$ theory, this behaviour with respect to finite $N$ will be called SEP-compatible.

Inverting (2.14) using character orthogonality relations gives

$$
T_{p}=\sum_{R \vdash n} \chi_{R}(p) s_{R}
$$

Combining this with (2.16) leads to a an expression for multi-traces in terms of monomials.

$$
T_{p}=\sum_{R, q \vdash n} \chi_{R}(p) K_{R q} m_{q}=\sum_{R, q \vdash n} \frac{\chi_{R}(p) K_{R q}}{\prod_{i} q_{i} !} M_{q}
$$

We will study this relationship in much greater depth in section 4, including the inverse relation giving monomials in terms of multi-traces. 


\subsubsection{Correlators}

The physical $\mathcal{N}=4$ inner product on multi-traces of the form (2.17) is given by

$$
\begin{aligned}
\left\langle\operatorname{Tr}\left(\tau X^{\otimes n}\right) \mid \operatorname{Tr}\left(\sigma X^{\otimes n}\right)\right\rangle=\left\langle T_{p_{\tau}} \mid T_{p_{\sigma}}\right\rangle & =\sum_{\alpha, \beta \in S_{n}} N^{c(\beta)} \delta\left(\alpha \sigma \alpha^{-1} \tau^{-1} \beta\right) \\
& =\sum_{\alpha \in S_{n}} \delta\left(\alpha \sigma \alpha^{-1} \tau^{-1} \Omega_{N}\right)
\end{aligned}
$$

where $p_{\sigma} \vdash n$ is the cycle type of $\sigma, c(\sigma)=l\left(p_{\sigma}\right)$ is the number of cycles in $\sigma$, and

$$
\Omega_{N}=\sum_{\sigma \in S_{n}} N^{c(\sigma)} \sigma
$$

We also use $\delta(\sigma)$, defined on $S_{n}$ by

$$
\delta(\sigma)= \begin{cases}1 & \sigma=1 \\ 0 & \text { otherwise }\end{cases}
$$

and extended linearly to the group algebra $\mathbb{C}\left(S_{n}\right)$.

Under conjugation of $\sigma$, the number of cycles $c(\sigma)$ is invariant, and therefore $\Omega_{N}$ commutes with any permutation in $S_{n}$

$$
\alpha \Omega_{N} \alpha^{-1}=\sum_{\sigma \in S_{n}} N^{c(\sigma)} \alpha \sigma \alpha^{-1}=\sum_{\sigma \in S_{n}} N^{c\left(\alpha^{-1} \sigma \alpha\right)} \sigma=\sum_{\sigma \in S_{n}} N^{c(\sigma)} \sigma=\Omega_{N}
$$

At large $N$, the leading $N$ behaviour of $\Omega_{N}$ is

$$
\Omega_{N}=N^{n}\left[1+O\left(\frac{1}{N}\right)\right]
$$

and consequently the inner product (2.21) reduces to $N^{n}$ times the $S_{n}$ inner product

$$
\left\langle\operatorname{Tr}\left(\tau X^{\otimes n}\right) \mid \operatorname{Tr}\left(\sigma X^{\otimes n}\right)\right\rangle_{S_{n}}=\left\langle T_{p_{\tau}} \mid T_{p_{\sigma}}\right\rangle_{S_{n}}:=\sum_{\alpha \in S_{n}} \delta\left(\alpha \sigma \alpha^{-1} \tau^{-1}\right)=\delta_{p_{\sigma} p_{\tau}} z_{p_{\sigma}}
$$

where we have used (2.15) for the size of the centraliser of $\sigma$ in order to evaluate the $S_{n}$ inner product.

When we take $S_{n}$ inner products of operators whose coefficients are $N$-independent, this is the same as the planar inner product. Therefore (2.26) shows that different multitraces are orthogonal in the planar limit with norm $z_{p}$. However in section 6 we consider operators whose coefficients depend on $N$. For these cases, (2.26) is distinct from the planar inner product, which would also take the large $N$ limit in coefficients.

Although the definition (2.26) was motivated by large $N$ considerations, it is also a valid definition at finite $N$. For $N<n$, it is useful to introduce the definition

$$
\delta_{N}(\sigma)=\frac{1}{n !} \sum_{\substack{R \vdash n \\ l(R) \leq N}} d_{R} \chi_{R}(\sigma)
$$


where $d_{R}$ is the dimension of the $S_{n}$ irreducible representation $R$. So a finite $N$ version of the $S_{n}$ inner product can be defined as

$$
\left\langle\operatorname{Tr}\left(\tau X^{\otimes n}\right) \mid \operatorname{Tr}\left(\sigma X^{\otimes n}\right)\right\rangle_{S_{n}}:=\sum_{\alpha \in S_{n}} \delta_{N}\left(\alpha \sigma \alpha^{-1} \tau^{-1}\right)
$$

The relation between the physical and $S_{n}$ inner products is determined by the properties of $\Omega_{N}$, which we now go through.

In a representation $R \vdash n, \Omega_{N}$ has representative

$$
D^{R}\left(\Omega_{N}\right)=\frac{n ! \operatorname{Dim}(R)}{d_{R}}=\prod_{b \in R}\left(N+c_{b}\right)=: f_{R}(N)
$$

where $\operatorname{Dim}(R)$ is the dimension of the $\mathrm{U}(N)$ irreducible representation $R$ and for a box $b$ in $R, c_{b}$ is the contents of $b$. If $b$ sits in the $r$ th row and $c$ th column of $R$, then $c_{b}=r-c$. For example if we take $R=[4,4,2]$, the contents of each box are

$$
\begin{array}{|c|c|c|c|}
\hline 0 & 1 & 2 & 3 \\
\hline-1 & 0 & 1 & 2 \\
\hline-2 & -1 & \multicolumn{2}{|c}{} \\
\cline { 1 - 2 } & &
\end{array}
$$

If $l(R)>N$, then $f_{R}=\operatorname{Dim}(R)=0$, and therefore $\Omega_{N}$ imposes the finite $N$ cut-off on Young diagrams.

Define the projector onto an irreducible representation $R$ of $S_{n}$ by

$$
P_{R}=\frac{d_{R}}{n !} \sum_{\sigma \in S_{n}} \chi_{R}(\sigma) \sigma
$$

This projects onto the $R$ representation of $S_{n}$ and commutes with all permutations in $S_{n}$. We can write $\Omega_{N}$ in terms of these projectors

$$
\Omega_{N}=\sum_{R \vdash n} f_{R} P_{R}
$$

Since $f_{R}=0$ for $R$ with $l(R)>N$, it follows that $\Omega_{N}$ is invertible only in those representations $R$ with $l(R) \leq N$. Define

$$
\Omega_{N}^{-1}=\sum_{\substack{R \vdash n \\ l(R) \leq N}} \frac{1}{f_{R}} P_{R}
$$

which has representatives

$$
D^{R}\left(\Omega_{N}^{-1}\right)= \begin{cases}\frac{1}{f_{R}} & l(R) \leq N \\ 0 & l(R)>N\end{cases}
$$

This is inverse to $\Omega_{N}$ in all representations $R$ with $l(R) \leq N$. If $N \geq n$, it is inverse to $\Omega_{N}$ in all irreducible representations of $S_{n}$, and is therefore inverse in the full group algebra $\mathbb{C}\left(S_{n}\right)$. 
Define maps $\mathcal{F}_{N}$ and $\mathcal{G}_{N}$ as versions of $\Omega_{N}$ and $\Omega_{N}^{-1}$ on gauge invariant operators

$$
\begin{aligned}
& \mathcal{F}_{N}\left[\operatorname{Tr}\left(\sigma X^{\otimes n}\right)\right]=\operatorname{Tr}\left(\Omega_{N} \sigma X^{\otimes n}\right) \\
& \mathcal{G}_{N}\left[\operatorname{Tr}\left(\sigma X^{\otimes n}\right)\right]=\operatorname{Tr}\left(\Omega_{N}^{-1} \sigma X^{\otimes n}\right)
\end{aligned}
$$

On operators, $\mathcal{F}_{N}$ and $\mathcal{G}_{N}$ are inverse to each other at all $N$.

It follows from (2.25) that at large $N, \mathcal{F}_{N}$ and $\mathcal{G}_{N}$ are multiplication and division by $N^{n}$ respectively. At finite $N$, they are more complicated operators.

Extending the definition linearly to generic degree $n$ operators $\mathcal{O}_{1}$ and $\mathcal{O}_{2}$, the relation between the physical and $S_{n}$ inner products is

$$
\begin{aligned}
\left\langle\mathcal{O}_{1} \mid \mathcal{O}_{2}\right\rangle & =\left\langle\mathcal{O}_{1} \mid \mathcal{F}_{N} \mathcal{O}_{2}\right\rangle_{S_{n}} \\
\left\langle\mathcal{O}_{1} \mid \mathcal{G}_{N} \mathcal{O}_{2}\right\rangle & =\left\langle\mathcal{O}_{1} \mid \mathcal{O}_{2}\right\rangle_{S_{n}}
\end{aligned}
$$

Motivated by the interaction of $\mathcal{F}_{N}$ and $\mathcal{G}_{N}$ with the different inner products, we call

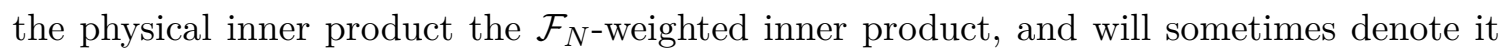
by adding a $\mathcal{F}$ subscript. We introduce a similar $\mathcal{G}$-weighted inner product

$$
\left\langle\mathcal{O}_{1} \mid \mathcal{O}_{2}\right\rangle_{\mathcal{G}}:=\left\langle\mathcal{O}_{1} \mid \mathcal{G}_{N} \mathcal{O}_{2}\right\rangle_{S_{n}}=\left\langle\mathcal{G}_{N} \mathcal{O}_{1} \mid \mathcal{G}_{N} \mathcal{O}_{2}\right\rangle_{\mathcal{F}}=\sum_{\alpha \in S_{n}} \delta\left(\alpha \sigma \alpha^{-1} \tau^{-1} \Omega_{N}^{-1}\right)
$$

The behaviour of multi-traces and monomials under $\mathcal{F}_{N}$ and $\mathcal{G}_{N}$ are in general quite complicated. The Schur operators (2.18) have much simpler properties, stemming from the expressions (2.29) and (2.34) for $\Omega_{N}$ and $\Omega_{N}^{-1}$ in the representation $R$.

$$
\begin{aligned}
\mathcal{F}\left(s_{R}\right) & =f_{R} s_{R} \\
\mathcal{G}_{N}\left(s_{R}\right) & = \begin{cases}\frac{1}{f_{R}} s_{R} & l(R) \leq N \\
0 & l(R)>N\end{cases}
\end{aligned}
$$

It follows these and the orthogonality of characters that

$$
\begin{aligned}
\left\langle s_{R} \mid s_{S}\right\rangle_{\mathcal{F}} & =\delta_{R S} f_{R} \\
\left\langle s_{R} \mid s_{S}\right\rangle_{S_{n}} & = \begin{cases}\delta_{R S} & l(R) \leq N \\
0 & l(R)>N\end{cases} \\
\left\langle s_{R} \mid s_{S}\right\rangle_{\mathcal{G}} & = \begin{cases}\delta_{R S} \frac{1}{f_{R}} & l(R) \leq N \\
0 & l(R)>N\end{cases}
\end{aligned}
$$

From their action on Schur operators, it is clear that $\mathcal{F}_{N}$ and $\mathcal{G}_{N}$ are Hermitian with respect to all three inner products.

The maps $\mathcal{F}_{N}$ and $\mathcal{G}_{N}$ were first defined in [32], where they were used to describe a general method of constructing weak coupling quarter-BPS operators. In section 7 we describe how that construction is related to the approach taken in this paper.

When evaluating correlators, we will often express answers as polynomials or rational functions in $N$. It will often be convenient to think of $N$ as a formal variable for these purposes. This is valid provided $n \leq N$. 
We give explicit examples of the monomial and Schur operators. Using the definitions (2.10) and (2.14) with $n=4$, we have

$$
\begin{aligned}
& M_{[4]}=m_{[4]}=\operatorname{Tr} X^{4} \\
& M_{[3,1]}=m_{[3,1]}=\operatorname{Tr} X \operatorname{Tr} X^{3}-\operatorname{Tr} X^{4} \\
& M_{[2,2]}=2 m_{[2,2]}=\left(\operatorname{Tr} X^{2}\right)^{2}-\operatorname{Tr} X^{4} \\
& M_{[2,1,1]}=2 m_{[2,1,1]}=(\operatorname{Tr} X)^{2} \operatorname{Tr} X^{2}-2 \operatorname{Tr} X \operatorname{Tr} X^{3}-\left(\operatorname{Tr} X^{2}\right)^{2}+2 \operatorname{Tr} X^{4} \\
& M_{[1,1,1,1]}=24 m_{[1,1,1,1]}=(\operatorname{Tr} X)^{4}-6(\operatorname{Tr} X)^{2} \operatorname{Tr} X^{2}+8 \operatorname{Tr} X \operatorname{Tr} X^{3} \\
&+3\left(\operatorname{Tr} X^{2}\right)^{2}-6 \operatorname{Tr} X^{4}
\end{aligned}
$$

and

$$
\begin{aligned}
s_{[4]} & =\frac{1}{24}\left[(\operatorname{Tr} X)^{4}+6(\operatorname{Tr} X)^{2} \operatorname{Tr} X^{2}+8 \operatorname{Tr} X \operatorname{Tr} X^{3}+3\left(\operatorname{Tr} X^{2}\right)^{2}+6 \operatorname{Tr} X^{4}\right] \\
s_{[3,1]} & =\frac{1}{8}\left[(\operatorname{Tr} X)^{4}+2(\operatorname{Tr} X)^{2} \operatorname{Tr} X^{2}-\left(\operatorname{Tr} X^{2}\right)^{2}-2 \operatorname{Tr} X^{4}\right] \\
s_{[2,2]} & =\frac{1}{12}\left[(\operatorname{Tr} X)^{4}-4 \operatorname{Tr} X \operatorname{Tr} X^{3}+3\left(\operatorname{Tr} X^{2}\right)^{2}\right] \\
s_{[2,1,1]} & =\frac{1}{8}\left[(\operatorname{Tr} X)^{4}-2(\operatorname{Tr} X)^{2} \operatorname{Tr} X^{2}-\left(\operatorname{Tr} X^{2}\right)^{2}+2 \operatorname{Tr} X^{4}\right] \\
s_{[1,1,1,1]} & =\frac{1}{24}\left[(\operatorname{Tr} X)^{4}-6(\operatorname{Tr} X)^{2} \operatorname{Tr} X^{2}+8 \operatorname{Tr} X \operatorname{Tr} X^{3}+3\left(\operatorname{Tr} X^{2}\right)^{2}-6 \operatorname{Tr} X^{4}\right]
\end{aligned}
$$

\subsection{Quarter-BPS covariant operators}

The zero coupling quarter-BPS sector is spanned by multi-traces of two complex matrices $X$ and $Y$. There are several natural extensions of the Schur basis (2.18) to the 2-matrix sector [27-29]. We will use the covariant basis, introduced in [25, 26], which is SEPcompatible and orthogonal to all orders in $N$. The basis elements are also referred to as BHR operators after the authors.

\subsection{1 $U(2)$ action on traces}

Let $X_{1}=X$ and $X_{2}=Y$. Then there is an action of $\mathrm{U}(2)$ on the $i$ index in $X_{i}$. By extension this acts on all traces and operators, so we can choose our basis to be $U(2)$ covariant. Since $\mathrm{U}(2)$ turns $X \mathrm{~s}$ into $Y \mathrm{~s}$ and vice versa, this basis mixes states with different numbers of $X \mathrm{~s}$ and $Y \mathrm{~s}$ while keeping the total number of matrices, $n$, constant. We will say an operator has field content $\left(n_{1}, n_{2}\right)$ if it contains $n_{1} X \mathrm{~s}$ and $n_{2} Y \mathrm{~s}$.

The $\mathfrak{u}(2)$ operators on traces are given by

$$
R_{j}^{i}=\left(\begin{array}{cc}
\mathcal{X} & \mathcal{J}_{+} \\
\mathcal{J}_{-} & \mathcal{Y}
\end{array}\right)=\left(\begin{array}{cc}
\operatorname{Tr} X \frac{\partial}{\partial X} & \operatorname{Tr} X \frac{\partial}{\partial Y} \\
\operatorname{Tr} Y \frac{\partial}{\partial X} & \operatorname{Tr} Y \frac{\partial}{\partial Y}
\end{array}\right)=\left(\begin{array}{cc}
X_{j}^{i} \frac{\partial}{\partial X_{j}^{i}} & X_{j}^{i} \frac{\partial}{\partial Y_{j}^{i}} \\
Y_{j}^{i} \frac{\partial}{\partial X_{j}^{i}} & Y_{j}^{i} \frac{\partial}{\partial Y_{j}^{i}}
\end{array}\right)
$$


The operator $\mathcal{X}$ counts the number of $X$ matrices in a trace, similarly for $\mathcal{Y}$. The lowering operator $\mathcal{J}_{-}$'lowers' a trace by turning an $X$ into a $Y$, and the raising operator $\mathcal{J}_{+}$'raises' a trace by turning a $Y$ into an $X$.

Acting on the matrices $X_{i}$ with a $\mathrm{U}(2)$ index

$$
R_{j}^{i} X_{k}=\delta_{k}^{i} X_{j}
$$

Define new operators

$$
\mathcal{J}_{0}=\mathcal{X}+\mathcal{Y} \quad \mathcal{J}_{3}=\mathcal{X}-\mathcal{Y}
$$

Then $\mathcal{J}_{0}$ counts the total number of matrices, while $\mathcal{J}_{3}$ counts the difference between the number of $X$ s and $Y$ s. As the notation suggests, $\mathcal{J}_{3}, \mathcal{J}_{ \pm}$form an $\mathfrak{s u}(2)$ subalgebra of $\mathfrak{u}(2)$, while $\mathcal{J}_{0}$ spans a $\mathfrak{u}(1)$ that commutes with the $\mathfrak{s u}(2)$. This split decomposes $\mathfrak{u}(2)$ into a sum of $\mathfrak{s u}(2)$ and $\mathfrak{u}(1)$.

The operators (2.54) obey standard hermiticity conditions $\left(R_{j}^{i}\right)^{\dagger}=R_{i}^{j}$ for $R$-symmetry generators

$$
\left(\mathcal{J}_{0}\right)^{\dagger}=\mathcal{J}_{0} \quad\left(\mathcal{J}_{3}\right)^{\dagger}=\mathcal{J}_{3} \quad\left(\mathcal{J}_{+}\right)^{\dagger}=\mathcal{J}_{-}
$$

It follows that operators with different $\mathrm{U}(2)$ quantum numbers must be orthogonal.

\subsubsection{U(2) representations}

Semi-standard Young tableaux are defined to be Young tableaux in which the positive integers in the boxes increase weakly along the rows and strictly down the columns. For

\begin{tabular}{|c|c|c|c|c|c|c|c|c|c|c|c|c|c|c|c|}
\hline 1 & 1 & 1 & 1 & 1 & 2 & 1 & 2 & 1 & 3 & 1 & 3 & 2 & 2 & 2 & 3 \\
\hline 2 & & 3 & & 2 & & 3 & & 2 & & 3 & & 3 & & 3 & \\
\hline
\end{tabular}
example if we take $R=[2,1]$ and allow entries of 1,2 and 3 , the possible semi-standard tableaux are:

The evaluation of a semi-standard tableau $r$ is a sequence of numbers $\rho(r)=\left[\rho_{1}, \rho_{2}, \ldots\right]$ where

$$
\rho_{i}=(\# \text { of occurences of the number } i \text { in } r)
$$

So for example the evaluations of the tableaux in (2.58) are respectively

$$
[2,1,0] \quad[2,0,1] \quad[1,2,0] \quad[1,1,1] \quad[1,1,1] \quad[1,0,2] \quad[0,2,1] \quad[0,1,2]
$$

When the evaluation $\rho(r)$ is a partition (i.e. $\rho_{1} \geq \rho_{2} \geq \ldots$ ), these tableaux contribute to the Kostka numbers $K_{R \rho}$ seen in (2.16).

For a representation $\Lambda \vdash n$ of $\mathrm{U}(2)$ with $l(\Lambda) \leq 2$, the basis vectors of $\Lambda$ are labelled by the semi-standard Young tableaux of shape $\Lambda$ containing only 1 s and 2 s. For $\Lambda=$ $\left[\frac{n}{2}+j, \frac{n}{2}-j\right]$, there are $2 j+1$ possible tableaux, where $j$ runs over the non-negative halfintegers up to $\frac{n}{2}$. These possibilities are

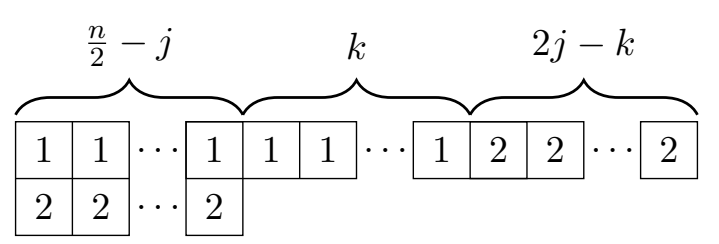


where $0 \leq k \leq 2 j$.

We can understand the representation $\Lambda=\left[\frac{n}{2}+j, \frac{n}{2}-j\right]$ in terms of the $\mathfrak{u}(1)$ spanned by $\mathcal{J}_{0}$ and the $\mathfrak{s u}(2)$ spanned by $\mathcal{J}_{3}, \mathcal{J}_{ \pm}$. All states in $\Lambda$ have weight $n$ under $\mathfrak{u}(1)$, and form a spin $j$ of $\mathfrak{s u}(2)$. The identification of basis vectors is

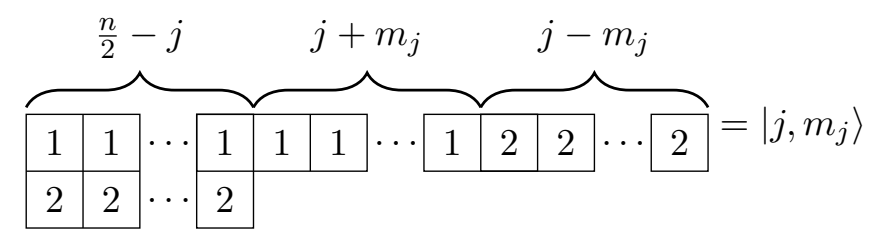

where $\left|j, m_{j}\right\rangle$ is the standard basis spanning the spin $j$ representation of $\mathfrak{s u}(2)$ with $-j \leq$ $m_{j} \leq j$.

\subsubsection{Operator definition}

To write down the covariant basis, consider $V_{2}^{\otimes n}$, where $V_{2}$ is the fundamental of $\mathrm{U}(2)$, and in particular the basis vector $a=e_{a_{1}} \otimes e_{a_{2}} \otimes \cdots \otimes e_{a_{n}}$ of $V_{2}^{\otimes n}$ where $a_{j} \in\{1,2\}$ for each $j$. Then we define $\mathbb{X}_{a}=X_{a_{1}} \otimes X_{a_{2}} \otimes \cdots \otimes X_{a_{n}}$. Combined with a permutation $\sigma \in S_{n}$, we write

$$
\mathcal{O}_{a, \sigma}=\operatorname{Tr}\left(\sigma \mathbb{X}_{a}\right)
$$

These operators are multi-traces, where each single trace factor corresponds to a cycle in $\sigma$.

The labelling set for the covariant basis is: $\Lambda \vdash n$, a partition with at most 2 rows; $M_{\Lambda}$, a semi-standard tableau of shape $\Lambda$ that indexes the basis vectors of the $\Lambda$ representation of $\mathrm{U}(2) ; R \vdash n$, a partition with at most $N$ rows; and $\tau$, a multiplicity index satisfying $1 \leq \tau \leq C(R, R, \Lambda)$, where $C(R, R, \Lambda)$ is the multiplicity of the trivial representation within $R \otimes R \otimes \Lambda$ (for $\Lambda$ as an $S_{n}$ representation), or equivalently the multiplicity of the $\Lambda$ representation within $R \otimes R$.

Using these labels, the BHR operators are defined by

$$
\mathcal{O}_{\Lambda, M_{\Lambda}, R, \tau}=\frac{\sqrt{d_{R}}}{n !} \sum_{\sigma, a, i, j, m} S_{i j m}^{R R \Lambda, \tau} D_{i j}^{R}(\sigma) C_{\Lambda, M_{\Lambda}, m}^{a} \mathcal{O}_{a, \sigma}
$$

where $D_{i j}^{R}(\sigma)$ is the $R$ representation matrix for $\sigma, C_{\Lambda, M_{\Lambda}, m}^{a}$ are the Clebsch-Gordon coefficients for the Schur-Weyl decomposition

$$
V_{2}^{\otimes n}=\bigoplus_{\substack{\Lambda \vdash n \\ l(\Lambda) \leq 2}} V_{\Lambda}^{\mathrm{U}(2)} \otimes V_{\Lambda}^{S_{n}}
$$

and $S_{i j m}^{R R \Lambda \tau}$ are the Clebsch-Gordon coefficients for the $\tau$ th copy of the trivial $S_{n}$ representation inside $R \otimes R \otimes \Lambda$.

The field content $\left(n_{1}, n_{2}\right)$ can be read off from the numbers in the tableau $M_{\Lambda}$. The number of $1 \mathrm{~s}$ in $M_{\Lambda}$ is $n_{1}$, while the number of $2 \mathrm{~s}$ is $n_{2}$.

When $\Lambda=[n]$ and $M_{\Lambda}$ is the tableau consisting only of 1 s, the operators (2.64) reduce to the standard Schur operators (2.18). Since other values of $M_{\Lambda}$ are related to these via 


\begin{tabular}{|c|c|c|c|c|c|c|c|c|c|c|}
\hline$M_{\Lambda}$ & \begin{tabular}{|l|l|l|l|}
1 & 1 & 2 & 2 \\
\end{tabular} & \begin{tabular}{|l|l|l|l|}
1 & 1 & 2 & 2 \\
\end{tabular} & \begin{tabular}{|l|l|l|l|}
1 & 1 & 2 & 2 \\
\end{tabular} & \begin{tabular}{|l|l|l|l|}
1 & 1 & 2 & 2 \\
\end{tabular} & \begin{tabular}{|l|l|l|l|}
1 & 1 & 2 & 2 \\
\end{tabular} & \begin{tabular}{|l|l|l|}
1 & 1 & 2 \\
2 & \\
\end{tabular} & \begin{tabular}{|l|l|l|}
$1 \frac{1}{2}$ & 2 \\
2
\end{tabular} & \begin{tabular}{|l|l|l|}
1 & 1 \\
2 & 2 \\
\end{tabular} & \begin{tabular}{|l|l|}
1 & 1 \\
2 & 2 \\
\end{tabular} & \begin{tabular}{|l|}
$\frac{1}{212}$ \\
22
\end{tabular} \\
\hline $\mathrm{R}$ & $\square \square \square$ & $\square$ & $\square$ & 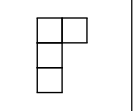 & $\theta$ & $\square$ & 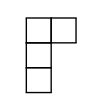 & $\square$ & $\square$ & 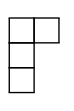 \\
\hline Normalisation & $\sqrt{6}$ & $\sqrt{6}$ & $\sqrt{6}$ & $\sqrt{6}$ & $\sqrt{6}$ & 1 & 1 & $\sqrt{3}$ & $\sqrt{6}$ & $\sqrt{3}$ \\
\hline Coefficient & $\overline{24}$ & $\overline{24}$ & $\overline{12}$ & $\overline{24}$ & $\overline{24}$ & $\overline{4}$ & $\overline{4}$ & $\overline{12}$ & $\overline{12}$ & $\overline{12}$ \\
\hline$(\operatorname{Tr} X)^{2}(\operatorname{Tr} Y)^{2}$ & 1 & 3 & 1 & 3 & 1 & 0 & 0 & 0 & 0 & 0 \\
\hline $\operatorname{Tr} X^{2}(\operatorname{Tr} Y)^{2}$ & 1 & 1 & 0 & -1 & -1 & 1 & -1 & 1 & -1 & -1 \\
\hline $\operatorname{Tr} X Y \operatorname{Tr} X \operatorname{Tr} Y$ & 4 & 4 & 0 & -4 & -4 & 0 & 0 & -2 & 2 & 2 \\
\hline$(\operatorname{Tr} X)^{2} \operatorname{Tr} Y^{2}$ & 1 & 1 & 0 & -1 & -1 & -1 & 1 & 1 & -1 & -1 \\
\hline $\operatorname{Tr} X^{2} Y \operatorname{Tr} Y$ & 4 & 0 & -2 & 0 & 4 & 2 & 2 & 0 & 0 & 0 \\
\hline $\operatorname{Tr} X Y^{2} \operatorname{Tr} X$ & 4 & 0 & -2 & 0 & 4 & -2 & -2 & 0 & 0 & 0 \\
\hline $\operatorname{Tr} X^{2} \operatorname{Tr} Y^{2}$ & 1 & -1 & 1 & -1 & 1 & 0 & 0 & 2 & 0 & 2 \\
\hline$(\operatorname{Tr} X Y)^{2}$ & 2 & -2 & 2 & -2 & 2 & 0 & 0 & -2 & 0 & -2 \\
\hline $\operatorname{Tr} X^{2} Y^{2}$ & 4 & -4 & 0 & 4 & -4 & 0 & 0 & 2 & 2 & -2 \\
\hline $\operatorname{Tr}(X Y)^{2}$ & 2 & -2 & 0 & 2 & -2 & 0 & 0 & -2 & -2 & 2 \\
\hline
\end{tabular}

Table 1. The covariant basis for field content $(2,2)$ in terms of multi-traces. Each element is identified by its $M_{\Lambda}$ and $R$ labels. We give the overall normalisation and the coefficient of each multi-trace within the operator.

$\mathfrak{u}(2)$ operators, which are part of the R-symmetry algebra, the entire $\Lambda=[n]$ sector is part of a half-BPS multiplet. The multiplicity index $\tau$ is trivial since $R \otimes R$ always contains a unique copy of the trivial representation

$$
C(R, R,[n])=1
$$

The $\Lambda=[n-1,1]$ sector also has special properties. In [40] it was proved that these multiplets cannot recombine to form long non-BPS multiplets and therefore must remain quarter-BPS at all values of the coupling. In these cases, the multiplicity $\tau$ in (2.64) runs over the number of corners of $R$ minus 1 .

$$
C(R, R,[n-1,1])=(\# \text { of corners in } R)-1
$$

This is proved most simply by comparing the covariant basis with the combinatorics of the restricted Schur basis defined in [28, 29].

The covariant basis is SEP-compatible, so when $N<n$, those operators with $l(R)>N$ vanish and the reduced set forms a basis for the space.

In table 1, we give explicit expressions for the $\mathrm{U}(2)$ covariant basis vectors with field content $(2,2)$. The equivalent expressions for field content $(4,0)$ were already given in (2.49)-(2.53). It's simple to check that one can travel between these different sectors using the $\mathrm{U}(2)$ operators $\mathcal{J}_{ \pm}$given in (2.54). 


\subsubsection{Correlators}

The two-point function of generic operators of the form (2.63) is given by [32].

$$
\left\langle\mathcal{O}_{b, \tau} \mid \mathcal{O}_{a, \sigma}\right\rangle=\sum_{\alpha \in S_{n}} \delta_{\alpha(a), b} \delta\left(\alpha \sigma \alpha^{-1} \tau^{-1} \Omega_{N}\right)
$$

The $\delta_{\alpha(a), b}$ factor restricts the sum over $\alpha$ to a subgroup $S_{n_{1}} \otimes S_{n_{2}}$ of $S_{n}$ for operators of field content $\left(n_{1}, n_{2}\right)$. Except for this restriction, the inner product looks very similar to the half-BPS case (2.21), and we proceed in an analogous manner. Define $\mathcal{F}_{N}$ and $\mathcal{G}_{N}$ by

$$
\mathcal{F}_{N} \mathcal{O}_{a, \alpha}=\mathcal{O}_{a, \Omega_{N} \alpha} \quad \mathcal{G}_{N} \mathcal{O}_{a, \alpha}=\mathcal{O}_{a, \Omega_{N}^{-1} \alpha}
$$

These are inverse to each other on finite $N$ operators. Define the $S_{n}$ and $\mathcal{G}$-weighted inner products on operators to be

$$
\begin{aligned}
\left\langle\mathcal{O}_{b, \tau} \mid \mathcal{O}_{a, \sigma}\right\rangle_{S_{n}} & :=\sum_{\alpha \in S_{n}} \delta_{\alpha(a), b} \delta_{N}\left(\alpha \sigma \alpha^{-1} \tau^{-1}\right)=\left\langle\mathcal{O}_{b, \tau} \mid \mathcal{G}_{N} \mathcal{O}_{a, \sigma}\right\rangle \\
\left\langle\mathcal{O}_{b, \tau} \mid \mathcal{O}_{a, \sigma}\right\rangle_{\mathcal{G}} & :=\sum_{\alpha \in S_{n}} \delta_{\alpha(a), b} \delta\left(\alpha \sigma \alpha^{-1} \tau^{-1} \Omega_{N}^{-1}\right)=\left\langle\mathcal{G}_{N} \mathcal{O}_{b, \tau} \mid \mathcal{G}_{N} \mathcal{O}_{a, \sigma}\right\rangle
\end{aligned}
$$

Then the three distinct inner products are related by multiplying operators by the appropriate $\mathcal{F}_{N}$ or $\mathcal{G}_{N}$.

The $\mathrm{U}(2)$ acts only on the label $a$ in $\mathcal{O}_{a, \sigma}$, while $\mathcal{F}_{N}$ and $\mathcal{G}_{N}$ act only on $\sigma$, and therefore the two commute. This means the same hermiticity conditions (2.57) apply in each of the physical, $S_{n}$ and $\mathcal{G}$-weighted inner products. Therefore operators with different $\mathrm{U}(2)$ quantum numbers are orthogonal in all three inner products.

The behaviour (2.29) of $\Omega_{N}$ in a representation $R$ of $S_{n}$ implies that

$$
\begin{aligned}
& \mathcal{F}_{N} \mathcal{O}_{\Lambda, M_{\Lambda}, R, \tau}=f_{R} \mathcal{O}_{\Lambda, M_{\Lambda}, R, \tau} \\
& \mathcal{G}_{N} \mathcal{O}_{\Lambda, M_{\Lambda}, R, \tau}= \begin{cases}\frac{1}{f_{R}} \mathcal{O}_{\Lambda, M_{\Lambda}, R, \tau} & l(R) \leq N \\
0 & l(R)>N\end{cases}
\end{aligned}
$$

and it follows [32] that the correlators of the covariant basis (2.64) are

$$
\begin{aligned}
\left\langle\mathcal{O}_{\Lambda, M_{\Lambda}, R, \tau} \mid \mathcal{O}_{\Lambda^{\prime}, M_{\Lambda^{\prime}}, R^{\prime}, \tau^{\prime}}\right\rangle & =\delta_{\Lambda, \Lambda^{\prime}} \delta_{M_{\Lambda}, M_{\Lambda^{\prime}}} \delta_{R, R^{\prime}} \delta_{\tau, \tau^{\prime}} f_{R} \\
\left\langle\mathcal{O}_{\Lambda, M_{\Lambda}, R, \tau} \mid \mathcal{O}_{\Lambda^{\prime}, M_{\Lambda^{\prime}}, R^{\prime}, \tau^{\prime}}\right\rangle_{S_{n}} & = \begin{cases}\delta_{\Lambda, \Lambda^{\prime}} \delta_{M_{\Lambda}, M_{\Lambda^{\prime}}} \delta_{R, R^{\prime}} \delta_{\tau, \tau^{\prime}} & l(R) \leq N \\
0 & l(R)>N\end{cases} \\
\left\langle\mathcal{O}_{\Lambda, M_{\Lambda}, R, \tau} \mid \mathcal{O}_{\Lambda^{\prime}, M_{\Lambda^{\prime}}, R^{\prime}, \tau^{\prime}}\right\rangle_{\mathcal{G}} & = \begin{cases}\frac{\delta_{\Lambda, \Lambda^{\prime}} \delta_{M_{\Lambda}, M_{\Lambda^{\prime}}} \delta_{R, R^{\prime}} \delta_{\tau, \tau^{\prime}}}{f_{R}} & l(R) \leq N \\
0 & l(R)>N\end{cases}
\end{aligned}
$$

\section{Quarter-BPS at weak coupling: key concepts}

In this section, we review some key concepts which form the background and motivation for the counting and construction of quarter-BPS operators at weak coupling developed in sections $4-7$. It is known that while the counting of quarter BPS operators jumps in going 
from zero coupling to first order in coupling, there are no further corrections to the counting in the transition to strong coupling [20]. It is further expected that some class of correlators of quarter-BPS operators are subject to non-renormalization theorems, analogous to those proved in the half-BPS sector (see [39] and references therein). Indeed one such class of correlators involving two half-BPS and one quarter BPS have been proved to be nonrenormalized [42]. We therefore expect that the finite $N$ counting and construction of quarter-BPS operators at weak coupling in SYM will reflect many properties of quarter BPS states arising from geometrical constructions at strong coupling SYM, or the dual weakly coupled gravity. These include quarter BPS giant gravitons and LLM geometries.

\subsection{Background on construction of quarter BPS operators}

When we turn the coupling on in $\mathcal{N}=4 \mathrm{SYM}$, some of the short quarter-BPS multiplets at zero coupling recombine and form long non-BPS multiplets. We give two equivalent ways of characterising which 2-matrix multi-trace operators remain quarter-BPS, and which do not.

Firstly, non-BPS multi-traces of $X, Y$ are SUSY descendants. It was explained in [22] that these are exactly commutator traces. That is, they are multi-traces (or linear combinations thereof) where at least one of the constituent single traces contains a commutator $[X, Y]$.

Secondly, consider the one-loop dilatation operator $[43,44]$

$$
\mathcal{H}_{2}=-\operatorname{Tr}\left([X, Y]\left[\frac{\partial}{\partial X}, \frac{\partial}{\partial Y}\right]\right)
$$

Quarter-BPS operators are annihilated by $\mathcal{H}_{2}$, and as $\mathcal{H}_{2}$ is hermitian in the free field inner product, they are orthogonal to the image. It is clear from the definition (3.1) that states in the image are commutator traces. While it is not immediately obvious that all commutator traces live in the image, our numerical calculations indicate that they are, and consistency with [22] implies they should be.

Therefore the multi-traces that remain quarter-BPS as we move to weak coupling are those that are orthogonal to the commutator traces under the physical free field inner product (2.68). This inner product is difficult to evaluate on multi-trace operators, so [32] took a different approach, using $\mathcal{F}_{N}$ and $\mathcal{G}_{N}$ defined in (2.73) to express the physical inner product in terms of the better understood $S_{n}$ inner product (2.75).

Let $\mathcal{O}^{c}$ be a commutator trace, and $\mathcal{O}^{s}$ be a pre-BPS operator, defined to be orthogonal to commutator traces under the $S_{n}$ inner product. Then using the relation (2.70) between the physical and $S_{n}$ inner products

$$
\left\langle\mathcal{O}^{c} \mid \mathcal{G}_{N} \mathcal{O}^{s}\right\rangle=\left\langle\mathcal{O}^{c} \mid \mathcal{O}^{s}\right\rangle_{S_{n}}=0
$$

So the operator $\mathcal{G}_{N} \mathcal{O}^{s}$ is a quarter-BPS operator at weak coupling.

The natural next step is to determine the form of the operators $\mathcal{O}^{s}$. It was demonstrated in [32] that for $N \geq n$ these are symmetrised traces. For a single trace, the symmetrised version is

$$
\operatorname{Tr}\left(X_{a_{1}} X_{a_{2}} \ldots X_{a_{n}}\right) \rightarrow \frac{1}{n !} \sum_{\sigma \in S_{n}} \operatorname{Tr}\left(X_{a_{\sigma(1)}} X_{a_{\sigma(2)}} \ldots X_{a_{\sigma(n)}}\right)=: \operatorname{Str}\left(X_{a_{1}} X_{a_{2}} \ldots X_{a_{n}}\right)
$$


where $a_{i} \in\{1,2\}$ for each $i$ and $X_{1}=X, X_{2}=Y$. For a multi-trace, this process is applied to each of the constituent single traces. A symmetrised trace is determined by the field content of each single trace factor. For a particular total field content $\left(n_{1}, n_{2}\right)$, the possible symmetrised traces are labelled by vector partitions $\mathbf{p}$. A vector partition is a set of integer 2 -vectors which sum to $\left(n_{1}, n_{2}\right)$, which we denote by $\mathbf{p} \vdash\left(n_{1}, n_{2}\right)$. For a vector partition $\mathbf{p}=\left[\left(k_{1}, l_{1}\right), \ldots\left(k_{m}, l_{m}\right)\right]$ the associated symmetrised trace is

$$
T_{\mathbf{p}}=\operatorname{Str}\left(X^{k_{1}} Y^{l_{1}}\right) \operatorname{Str}\left(X^{k_{2}} Y^{l_{2}}\right) \ldots \operatorname{Str}\left(X^{k_{m}} Y^{l_{m}}\right)
$$

We conclude a generic quarter-BPS operator for $N \geq n$ can simply be written as

$$
\mathcal{O}^{\mathrm{BPS}}=\mathcal{G}_{N} T_{\mathbf{p}}
$$

At finite $N$, non-trivial relations among different multi-traces reduce the dimensionality of the quarter-BPS sector, and correspondingly the pre-BPS operators as well. A finite $N$ relation among traces could have three distinct behaviours with respect to the large $N$ space of symmetrised traces

1. It is internal to the space of symmetrised traces. In this case, under an appropriate choice of basis, a symmetrised trace reduces to the zero operator. Correspondingly, the dimension of the pre-BPS and BPS sectors reduce by 1 .

2. It is internal to the space of commutator traces. This does not affect the pre-BPS or BPS sectors.

3. It is a linear combination of symmetrised traces and commutator traces. In this case, under an appropriate choice of basis, a symmetrised trace reduces to a commutator trace. This means it is no longer pre-BPS, as it is not $S_{n}$ orthogonal to descendants. Correspondingly, the dimension of the pre-BPS and BPS sectors reduce by 1 .

Therefore, SEP-compatibility in the pre-BPS sector has a different interpretation to the BPS equivalent. A basis for pre-BPS operators is SEP-compatible if operators with labels longer than $N$ reduce to either the zero operator or a commutator trace after applying finite $N$ relations. After applying $\mathcal{G}_{N}$ to such a basis, we obtain an SEP-compatible basis for the quarter-BPS sector.

\subsection{Steps in the construction of an SEP-compatible orthogonal BPS basis}

The key ingredient that will allow the construction of an SEP-compatible basis for preBPS operators is an isomorphism proved by Vaccarino [35] and Domokos [36], summarised nicely by Procesi in [37].

From the definition (3.3), the non-commuting matrices $X$ and $Y$ commute within a symmetrised trace, and therefore we naively expect that symmetrised traces of noncommuting matrices correspond to ordinary multi-traces of commuting matrices via

$$
\operatorname{Str}\left(X^{k_{1}} Y^{l_{1}}\right) \ldots \operatorname{Str}\left(X^{k_{m}} Y^{l_{m}}\right) \longleftrightarrow \operatorname{Tr}\left(A^{k_{1}} B^{l_{1}}\right) \ldots \operatorname{Tr}\left(A^{k_{m}} B^{l_{m}}\right)
$$


where $A$ and $B$ are two commuting $N \times N$ matrices. The isomorphism of [35-37] makes this expectation rigorous.

Consider the ring $R(X, Y)$ generated by the matrix elements of two $N \times N$ matrices $X, Y$. This ring is acted on by $\mathrm{U}(N)$ via simultaneous conjugation of the two matrices. Given a $\mathcal{U} \in \mathrm{U}(N)$, we have

$$
(X, Y) \rightarrow\left(\mathcal{U} X \mathcal{U}^{\dagger}, \mathcal{U} Y \mathcal{U}^{\dagger}\right)
$$

Then invariants of $R(X, Y)$ under this action are multi-traces of $X$ and $Y$, and correspond to the quarter-BPS sector at zero coupling. At weak coupling, we consider $R(X, Y)$ modulo the ideal $I$ generated by the commutator $[X, Y]$. We call the quotient ring $R_{s}(X, Y)$. Each $\mathrm{U}(N)$ invariant of the quotient ring corresponds to an equivalence class of multi-trace operators related by addition of a commutator trace. In each class there is a unique pre-BPS representative that is orthogonal to all commutator traces (under the $S_{n}$ inner product). There is also a unique BPS operator orthogonal to commutator traces under the $\mathcal{F}_{N}$ inner product. Conversely, given a pre-BPS or BPS operator, there is a unique equivalence class to which it belongs. Therefore the invariants of $R_{s}(X, Y)$ give the combinatorics of the pre-BPS and BPS sectors, both at large $N$ and finite $N$.

Finding the pre-BPS operator in a given equivalence class is simple when $N \geq n$; as discussed in section 3.1, the representative is a symmetrised trace. When $N<n$, the multitrace expansion of an operator is non-unique, and it is more difficult to identify the pre-BPS operator. In section 6 , we describe how to find the pre-BPS operator by orthogonalisation.

On the other side of the isomorphism are multi-symmetric functions. Take two commuting $N \times N$ matrices $A=\operatorname{Diag}\left(x_{1}, x_{2}, \cdots, x_{N}\right)$ and $B=\operatorname{Diag}\left(y_{1}, y_{2}, \cdots, y_{N}\right)$. Then a multi-trace of $A$ and $B$ will be a polynomial in the $2 N$ variables invariant under permutations

$$
\left(x_{i}, y_{i}\right) \rightarrow\left(x_{\sigma(i)}, y_{\sigma(i)}\right)
$$

for $\sigma \in S_{N}$. These are called multi-symmetric functions, and generalise the symmetric functions of section 2.1 to two families of variables. They are discussed in detail in section 4 .

The theorem in [35-37] tells us that the ring of invariants of $R_{s}(X, Y)$ is isomorphic to the ring of multi-symmetric functions in $2 N$ variables.

This isomorphism is simple to give explicitly. Take a multi-trace of $X$ and $Y$ and restrict the two matrices to be diagonal. This is now a multi-symmetric function in the $2 N$ eigenvalues. Clearly the commutator $[X, Y]$ vanishes for the diagonal $X$ and $Y$, and therefore any multi-traces related by a commutator trace lead to the same multisymmetric function.

Conversely, given a multi-trace of two commuting matrices $A$ and $B$, we use the map (3.6) to pick a representative of the isomorphic equivalence class. At large $N$, this correctly identifies the pre-BPS operator. However, for $N<n$, this map does not associate a unique symmetrised trace with a given multi-symmetric function. Finite $N$ relations mean a multi-symmetric function can be written in multiple ways as the trace of commuting matrices. These different expressions give genuinely different operators in the gauge theory, related by commutator traces. For the multi-symmetric functions we use in this 
paper, we will give a defining representation as a multi-trace of commuting matrices, and then (3.6) defines the equivalent symmetrised trace operator in an unambiguous way.

We will generally use the same notation for either side of the isomorphism. For example we will use $X$ and $Y$ to refer to both the commuting matrices on the right of (3.6) and the non-commuting matrices of the super Yang-Mills theory on the left. Similarly, both a symmetrised trace and its isomorphic multi-symmetric function will be denoted $T_{\mathbf{p}}$. When the distinction is important, we will be clear which is under discussion.

For symmetric functions, we introduced two SEP-compatible bases, the monomial basis of section 2.1.1 and the Schur basis of section 2.1.3. There is no obvious analogue of the Schur basis for multi-symmetric functions, however the monomial basis does generalise, and provides a good finite $N$ description for multi-symmetric functions. We denote these monomials by $M_{\mathbf{p}}$, where the label is a vector partition $\mathbf{p}$, as already seen for symmetrised traces in (3.4). The length of $\mathbf{p}$ determines the finite $N$ behaviour. In section 4 we study the basis change between $T_{\mathbf{p}}$ and $M_{\mathbf{p}}$, both as multi-symmetric functions and their isomorphic image as symmetrised trace operators.

Under the map (3.6), the $M_{\mathbf{p}}$ give a basis for pre-BPS operators for $N \geq n$. When $N<n$, the operators with $l(\mathbf{p})>N$ reduce to commutator traces. As discussed in section 3.1, this is a feature of an SEP-compatible basis of pre-BPS operators. However, the operators with $l(\mathbf{p}) \leq N$ are not $S_{n}$ orthogonal to all commutator traces, and therefore are not pre-BPS. This is because the map (3.6) did not choose the correct pre-BPS operator from the equivalence class of operators related by addition of a commutator trace. We say $M_{\mathbf{p}}$ is SEP-compatible modulo commutators for the pre-BPS sector, and this is a key stepping stone to an SEP-compatible basis.

In section 5 we organise the $M_{\mathbf{p}}$ according to representations of the $\mathrm{U}(2)$ symmetry, replacing the label $\mathbf{p}$ with $\left(\Lambda, M_{\Lambda}, p, \nu\right) . \Lambda$ is a $\mathrm{U}(2)$ Young diagram with $n$ boxes, where $n$ is the total number of $X, Y$ matrices in the operator. $M_{\Lambda}$ labels a basis state in the $\Lambda$ representation of $\mathrm{U}(2) . p$ is an integer partition of $n$ whose components are related to the vector partition $\mathbf{p}$ simply by summing each of the two-vector components of $\mathbf{p}$. We call $p$ the associated partition of $\mathbf{p}$. Since $l(\mathbf{p})=l(p)$, the SEP-compatibility (modulo commutators) of $M_{\mathbf{p}}$ is transferred to the covariant basis $M_{\Lambda, M_{\Lambda}, p, \nu}$. This restricts $l(p) \leq N$, which is the usual constraint associated with a $\mathrm{U}(N)$ Young diagram, and we will therefore refer to $p$ as a $\mathrm{U}(N)$ Young diagram label. The final label $\nu$ runs over a multiplicity space of dimension $\mathcal{M}_{\Lambda, p}$. Much of section 5 is devoted to calculating and understanding $\mathcal{M}_{\Lambda, p}$ as it describes the finite $N$ combinatorics of the weak coupling quarter-BPS sector.

For $N \geq n$, the operators $T_{\mathbf{p}}, M_{\mathbf{p}}$ or $M_{\Lambda, M_{\Lambda}, p, \nu}$ each form bases of the pre-BPS space and their $\mathcal{G}_{N}$-images are BPS bases. These are denoted by $T_{\mathbf{p}}^{\mathrm{BPS}}, M_{\mathbf{p}}^{\mathrm{BPS}}$ and $M_{\Lambda, M_{\Lambda}, p, \nu}^{\mathrm{BPS}}$ respectively (in addition to applying $\mathcal{G}_{N}$, these operators involve normalising with respect to the $S_{n}$ inner product, a process discussed in detail in section 6.3). The properties of these bases, along with others we will intorduce momentarily, are shown in table 2 .

Section 6 starts with the covariant monomials $M_{\Lambda, M_{\Lambda}, p, \nu}$ and explicitly constructs a BPS, SEP-compatible, orthogonal basis $S_{\Lambda, M_{\Lambda}, p, \nu}^{\mathrm{BPS}}$ at any value of $N$. This algorithm varies in complexity depending on $N$. When $N \geq n$ it is fairly simple, while for $N<n$ it is significantly more complicated. 


\begin{tabular}{|c|c|c|c|c|}
\hline & SEP-compatible & $\mathrm{U}(2)$ covariant & BPS at $N<n$ & Orthogonal \\
\hline$T_{\mathbf{p}}^{\text {BPS }}$ & $\times$ & $\times$ & $\times$ & $\times$ \\
\hline$M_{\mathbf{p}}^{\text {BPS }}$ & modulo commutators & $\times$ & $\times$ & $\times$ \\
\hline$M_{\Lambda, M_{\Lambda}, p, \nu}^{\text {BPS }}$ & modulo commutators & $\checkmark$ & $\times$ & $\times$ \\
\hline $\bar{M}_{\Lambda, M_{\Lambda}, p, \nu}^{\text {BPS }}$ & modulo commutators & $\checkmark$ & $\checkmark$ & $\times$ \\
\hline$S_{\Lambda, M_{\Lambda}, p, \nu}^{\text {BPS }}$ & $\checkmark$ & $\checkmark$ & $\checkmark$ & $\checkmark$ \\
\hline
\end{tabular}

Table 2. Properties of the different BPS bases constructed. All are BPS at $N \geq n$.

When $N \geq n, S_{\Lambda, M_{\Lambda}, p, \nu}^{\mathrm{BPS}}$ are obtained by orthogonalising the BPS covariant monomials

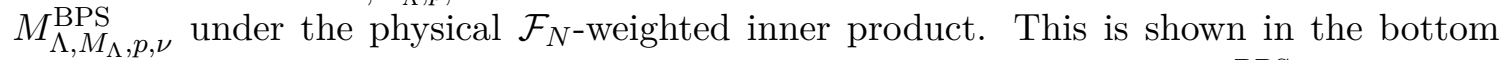
right of figure 1 , which also outlines the processes necessary to derive $S_{\Lambda, M_{\Lambda}, p, \nu}^{\mathrm{BPS}}$ starting from symmetrised trace operators $T_{\mathbf{p}}$. Due to the linear nature of these processes, they commute with the application of $\mathcal{G}_{N}$, and consequently there is flexibility in when this step is performed. We have written an implementation of this algorithm SAGE [74] which proceeds down the left hand side and across the bottom of figure 1. This code is used in section 6 and appendices A-C to generate explicit BPS operators.

When $N<n$, the process is more involved, as the covariant monomial $M_{\Lambda, M_{\Lambda}, p, \nu}$ $(l(p) \leq N)$ differs from a pre-BPS operator by a commutator trace. The process here is illustrated in figure 2 and involves the following three steps.

1. Orthogonalise $M_{\Lambda, M_{\Lambda}, p, \nu}$ against those $M_{\Lambda, M_{\Lambda}, q, \eta}$ with $l(q)>N$ using the $S_{n}$ inner product. This identifies the pre-BPS operator $\bar{M}_{\Lambda, M_{\Lambda}, p, \nu}$ in the equivalence class of operators related by commutator traces.

2. Apply $\mathcal{G}_{N}$ to the pre-BPS operators $\bar{M}_{\Lambda, M_{\Lambda}, p, \nu}$ to produce BPS operators $\bar{M}_{\Lambda, M_{\Lambda}, p, \nu}^{\mathrm{BPS}}$ (up to normalisation under the $S_{n}$ inner product).

3. Orthogonalize the BPS operators $\bar{M}_{\Lambda, M_{\Lambda}, p, \nu}^{\mathrm{BPS}}$ using the physical $\mathcal{F}_{N}$ inner product and normalise the output using the $S_{n}$ inner product.

The first step is explained in detail in section 7 in a more general context where the 2matrix problem is generalized to allow any number of matrices. Section 6 explores each step in detail, puts them together, and proves that the outcome $S_{\Lambda, M_{\Lambda}, p, \nu}^{\mathrm{BPS}}$ indeed form an orthogonal SEP-compatible basis for BPS operators.

In the case where $\Lambda$ is taken to be $[n]$, the $S_{\Lambda, M_{\Lambda}, p, \nu}^{\mathrm{BPS}}$ are half-BPS operators built purely from $X$. In section 6.9 we show that the construction for Young diagram $p$ reproduces the Schur operator (2.18) labelled by Young diagram $R=p$. Further, for $\Lambda=[n-1,1]$, numerical calculations suggest that $S_{\Lambda, M_{\Lambda}, p, \nu}^{\mathrm{BPS}}$ match the free field quarter-BPS operators (2.64) with $R=p$. This justifies the view that $p$ is a $\mathrm{U}(2)$ invariant, quarter-BPS generalization of the $R$-label of the half-BPS sector.

An important perspective on the half-BPS $R$ label comes from the analysis of the asymptotics of LLM geometries. Specifically $\mathrm{U}(N)$ Casimirs of $R$ are measurable from the 


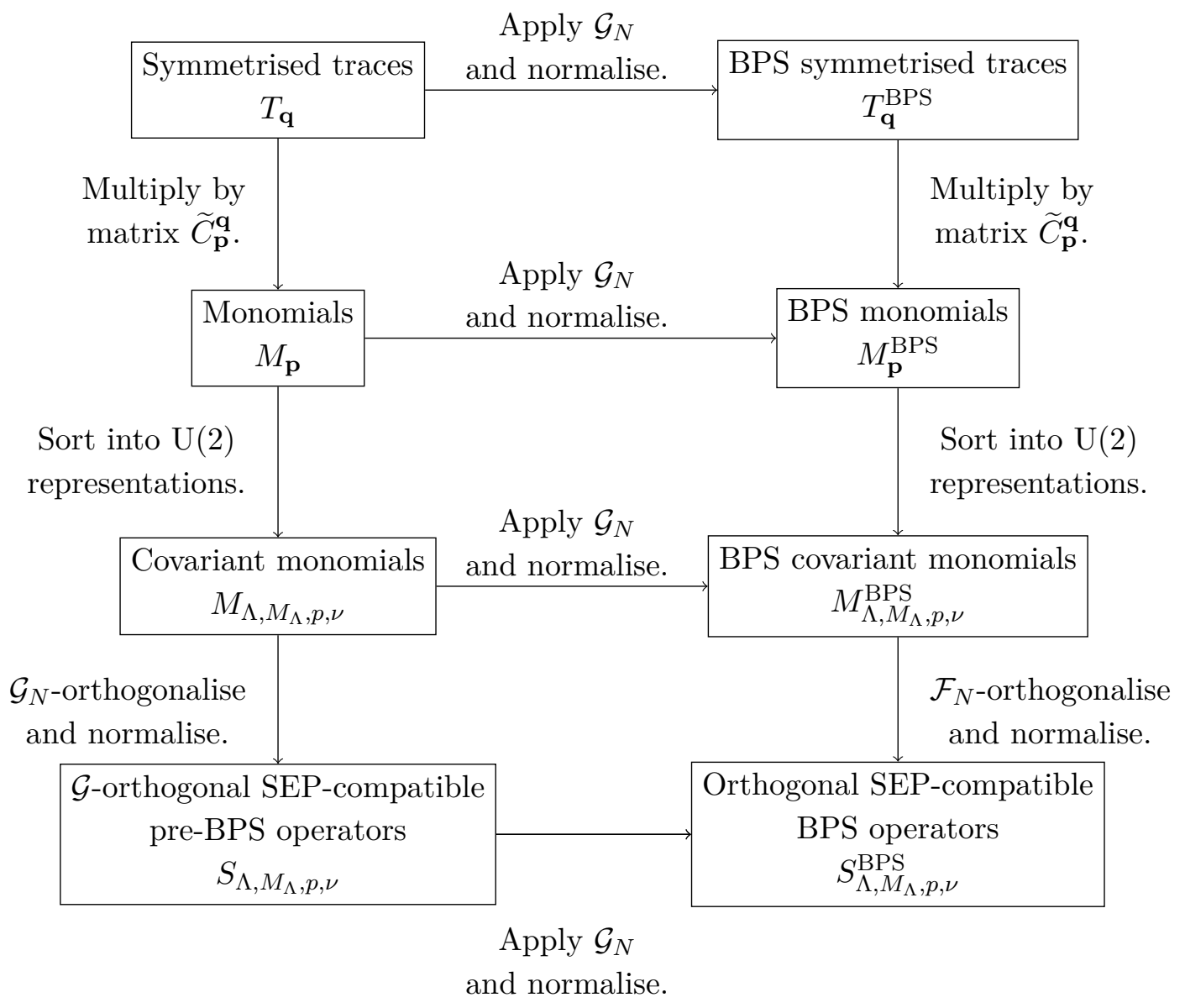

Figure 1. An outline of the algorithm presented in this paper starting with symmetrised trace operators $T_{\mathbf{p}}$ and deriving a U(2) covariant, orthogonal, SEP-compatible basis $S_{\Lambda, M_{\Lambda}, p, \nu}^{\mathrm{BPS}}$ for BPS operators when $N \geq n$. All normalisations are with respect to the $S_{n}$ inner product. Note that any algorithm to derive $S_{\Lambda, M_{\Lambda}, p, \nu}^{\mathrm{BPS}}$ need only involve one normalisation step at the very end. The extra normalisation steps are included in the figure in case the desired output is a different choice of BPS basis such as $M_{\Lambda, M_{\Lambda}, p, \nu}^{\mathrm{BPS}}$.

asymptotics of the supergravity fields [45]. We propose that the $\mathrm{U}(2)$ quadratic Casimir for $\Lambda$ as well as the sequence of Casimirs identifying $p$ should be measurable from the multipole moments that can be read off from the long-distance expansions of the sugra fields of LLM geometries corresponding to quarter-BPS operators at $n \sim N^{2}$. Precision holography of LLM geometries is also developed using correlators of small operators in the LLM background [46] which should provide complementary insights into the labels $\Lambda, p$.

The $R$ Young diagram label of the half BPS sector is related to the free fermions underlying the holomorphic sector of the complex matrix model which describes half-BPS combinatorics $[9,47,48]$. Remarkably, this free fermion description also shows up in the droplet description of half-BPS LLM geometries [7]. The droplet description generalizes to the quarter-BPS LLM geometries, with some significant differences [49-51]. We have colourings of regions in a four-dimensional space instead of a two-dimensional plane. The 


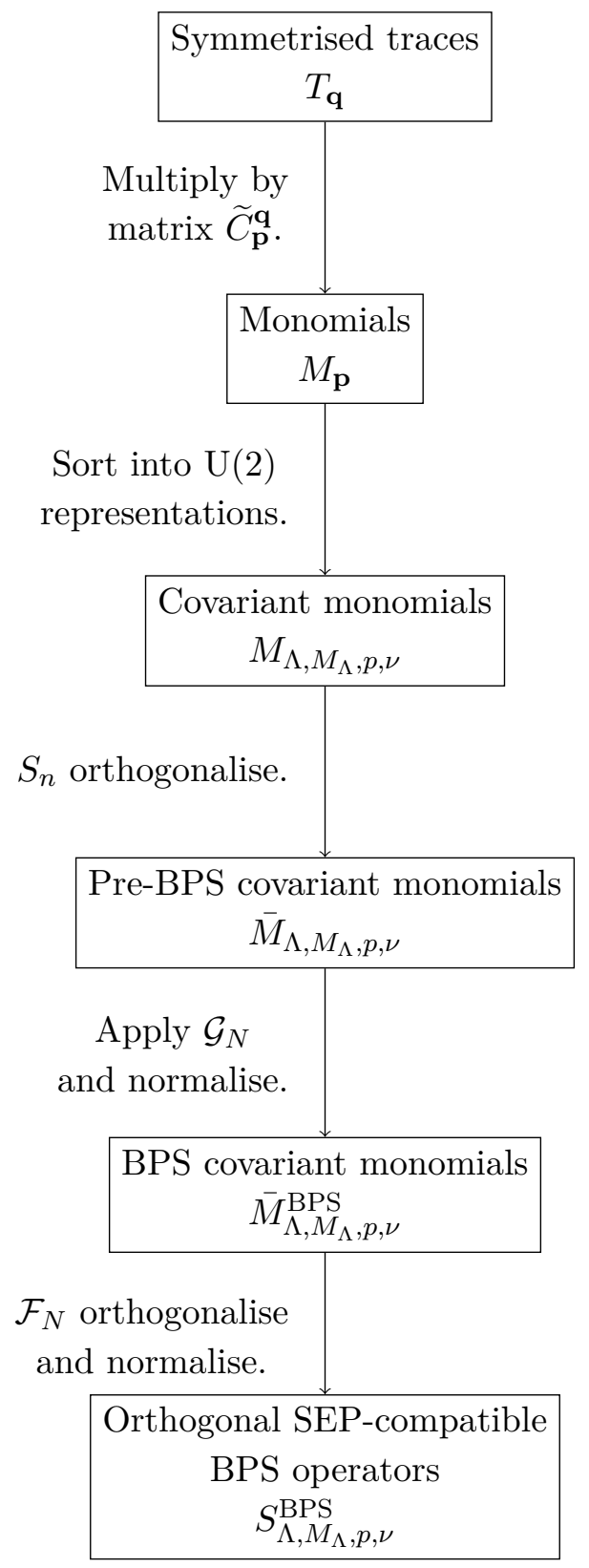

Figure 2. An outline of the algorithm presented in this paper starting with symmetrised trace operators $T_{\mathbf{p}}$ and deriving a U(2) covariant, orthogonal, SEP-compatible basis $S_{\Lambda, M_{\Lambda}, p, \nu}^{\mathrm{BPS}}$ for BPS operators when $N<n$. The first step is studied in detail in section 4 , the second step in section 5 and the last three steps in section 6 . All normalisations are with respect to the $S_{n}$ inner product. Note that any algorithm to derive $S_{\Lambda, M_{\Lambda}, p, \nu}^{\mathrm{BPS}}$ need only involve one normalisation step at the very end. The extra normalisation step is included in the figure in case the desired output is $\bar{M}_{\Lambda, M_{\Lambda}, p, \nu}^{\mathrm{BPS}}$. 
two colours are now associated with the collapse of an $S^{1} \subset S^{5}$ or an $S^{3} \subset S^{5}$. A natural conjecture is that the $p$-label of quarter-BPS operators is analogously associated to colourings of regions in $\mathbb{R}^{4}$ as the $R$-label of the half-BPS operators is associated to colourings of the plane.

\section{Finite $\mathrm{N}$ combinatorics from many-boson states: multi-symmetric functions and set partitions}

As explained in section 3, the key result that will enable us to give an SEP-compatible construction of quarter-BPS operators is an isomorphism of Vaccarino and Domokos [35, 36] between multi-symmetric functions and the ring of gauge invariants of two matrices modulo commutator traces. This section focuses on the multi-symmetric function side of this isomorphism.

An important aspect of multi-symmetric functions, which plays a central role in this paper, is the transformation between two bases for these multi-symmetric functions. The first basis will be referred to as the "monomial multi-symmetric basis" and the second as the "multi-trace basis". The physical importance of these two bases, and their transformations, can be understood using perspectives from many-body quantum mechanics [52]. This draws on an important insight from the AdS/CFT correspondence: the strong coupling limit of the quarter BPS sector in $\mathcal{N}=4 \mathrm{SYM}$ corresponds to a Hilbert space of $N$ bosons in a two-dimensional harmonic oscillator [17-19].

We begin this section by developing the link between multi-symmetric functions and the Hilbert space of $N$ identical bosons in a two-dimensional harmonic oscillator. We then introduce the monomial and multi-trace bases and investigate the combinatorics of the matrix that transforms between the two. This leads to the interesting mathematics of the poset of set partitions and the associated Möbius function.

\subsection{Multi-symmetric functions as wavefunctions of a harmonic oscillator}

The Lagrangian for one particle in a two-dimensional harmonic oscillator is

$$
L=\frac{1}{2}\left(\dot{x}^{2}+\dot{y}^{2}\right)-\frac{1}{2}\left(x^{2}+y^{2}\right)
$$

In terms of creation and annihilation operators, the one-particle Hamiltonian is

$$
H=a_{x}^{\dagger} a_{x}+a_{y}^{\dagger} a_{y}
$$

Define the coherent state

$$
\langle x, y|=\langle 0| e^{x a_{x}+y a_{y}}
$$

We have

$$
\left\langle x, y\left|\left(a_{x}^{\dagger}\right)^{\lambda}\left(a_{y}^{\dagger}\right)^{\mu}\right| 0\right\rangle=x^{\lambda} y^{\mu}
$$


In this coherent state representation, the Hamiltonian acts as the degree operator for the 2 -variable polynomial

$$
\begin{aligned}
\left\langle x, y\left|H\left(a_{x}^{\dagger}\right)^{\lambda}\left(a_{y}^{\dagger}\right)^{\mu}\right| 0\right\rangle & =\left(x \frac{\partial}{\partial x}+y \frac{\partial}{\partial y}\right)\left\langle x, y\left|\left(a_{x}^{\dagger}\right)^{\lambda}\left(a_{y}^{\dagger}\right)^{\mu}\right| 0\right\rangle \\
& =\left(x \frac{\partial}{\partial x}+y \frac{\partial}{\partial y}\right)\left(x^{\lambda} y^{\mu}\right) \\
& =(\lambda+\mu)\left(x^{\lambda} y^{\mu}\right)
\end{aligned}
$$

For the system of $N$-particles in the two-dimensional harmonic oscillator, we have the coherent state

$$
\left\langle x_{1}, y_{1} ; x_{2}, y_{2} ; \ldots ; x_{N}, y_{N}\right|=\langle 0| e^{\sum_{i=1}^{N} x_{i} a_{i ; x}+y_{i} a_{i ; y}}
$$

The energy eigenstates of the Hamiltonian correspond to the product of one-particle wavefunctions

$$
\begin{gathered}
\left\langle x_{1}, y_{1} ; x_{2}, y_{2} ; \cdots ; x_{N}, y_{N}\left|\left(a_{1 ; x}^{\dagger}\right)^{\lambda_{1}}\left(a_{1 ; y}^{\dagger}\right)^{\mu_{1}}\left(a_{2 ; x}^{\dagger}\right)^{\lambda_{2}}\left(a_{2 ; y}^{\dagger}\right)^{\mu_{2}} \ldots\left(a_{k ; x}^{\dagger}\right)^{\lambda_{k}}\left(a_{k ; y}^{\dagger}\right)^{\mu_{k}}\right| 0\right\rangle \\
=x_{1}^{\lambda_{1}} y_{1}^{\mu_{1}} \cdots x_{k}^{\lambda_{k}} y_{k}^{\mu_{k}}
\end{gathered}
$$

It is useful to write

$$
\begin{aligned}
x_{1}^{\lambda_{1}} y_{1}^{\mu_{1}} \ldots x_{k}^{\lambda_{k}} y_{k}^{\mu_{k}} & =\psi_{\lambda_{1}, \mu_{1}}\left(x_{1}, y_{1}\right) \psi_{\lambda_{2}, \mu_{2}}\left(x_{2}, y_{2}\right) \cdots \psi_{\lambda_{k}, \mu_{k}}\left(x_{k}, y_{k}\right) \\
& =\psi_{\lambda_{1}, \mu_{1}}\left(x_{1}, y_{1}\right) \ldots \psi_{\lambda_{k}, \mu_{k}}\left(x_{k}, y_{k}\right) \psi_{0,0}\left(x_{k+1}, y_{k+1}\right) \ldots \psi_{0,0}\left(x_{N}, y_{N}\right)
\end{aligned}
$$

In a system of $N$ identical bosons, we must symmetrise the product of annihilation operators using $S_{N}$ permutations. The product wavefunctions and their symmetrisations are a standard tool in many-body quantum mechanics (see. e.g. [52]). The permutations $\sigma \in S_{N}$ act as

$$
\left(x_{i}, y_{i}\right) \rightarrow\left(x_{\sigma(i)}, y_{\sigma(i)}\right)
$$

These states are polynomials, symmetric under these simultaneous permutations of $x, y$ pairs, which are exactly multi-symmetric functions. In fact these form the monomial multisymmetric functions that we will study presently. They have the nice property that finite $N$ effects are nicely encoded in the fact that, by definition, $k \leq N$.

A quantum state where a single particle is excited, after symmetrisation, has a coherent state representation

$$
\phi_{\lambda_{1}, \mu_{1}}\left(x_{i}, y_{i}\right)=\sum_{i=1}^{N} x_{i}^{\lambda_{1}} y_{i}^{\mu_{1}}
$$

When we have two particles excited, the symmetrisation of the product wavefunction is proportional to

$$
\sum_{i_{1} \neq i_{2}}^{N} x_{i_{1}}^{\lambda_{1}} y_{i_{1}}^{\mu_{1}} x_{i_{2}}^{\lambda_{2}} y_{i_{2}}^{\mu_{2}}
$$

The restriction $i_{1} \neq i_{2}$, when extended to $i_{1} \neq i_{2} \cdots \neq i_{k}$, is closely related to the finite $N$ property, but also has the consequence that the 2-particle wavefunction (4.11) is not equal 
to the product of 1-particle wavefunctions. It is rather a linear combination of the product wavefunction $\phi_{\lambda_{1}, \mu_{1}} \phi_{\lambda_{2}, \mu_{2}}$ along with a 1-particle wavefunction $\phi_{\lambda_{1}+\lambda_{2}, \mu_{1}+\mu_{2}}$.

Defining diagonal matrices $X=\operatorname{Diag}\left(x_{1}, \cdots, x_{N}\right)$ and $Y=\operatorname{Diag}\left(y_{1}, y_{2}, \cdots, y_{N}\right)$, we observe that the 1-particle wavefunction is a trace

$$
\phi_{\lambda_{1}, \mu_{1}}\left(x_{i}, y_{i}\right)=\operatorname{Tr}\left(X^{\lambda_{1}} Y^{\mu_{1}}\right)
$$

We now draw on an idea from collective field theory, where one associates creation operators to invariant traces [53-55] to define a map from traces and products of traces to Fock space states

$$
\begin{aligned}
\operatorname{Tr}\left(X^{\lambda} Y^{\mu}\right) & \rightarrow B_{\lambda, \mu}^{\dagger}|0\rangle \\
\prod_{a=1}^{k} \operatorname{Tr}\left(X^{\lambda_{a}} Y^{\mu_{a}}\right) & \rightarrow \prod_{a=1}^{k} B_{\lambda_{a}, \mu_{a}}^{\dagger}|0\rangle
\end{aligned}
$$

This map is a homomorphism between the product structure on the polynomials and the product structure on oscillators. It can also be obtained from a coherent state construction

$$
\begin{aligned}
\langle X, Y| & =\langle 0| e^{\sum_{\lambda, \mu} B_{\lambda, \mu} \operatorname{Tr}\left(X^{\lambda} Y^{\mu}\right)} \\
\left\langle X, Y\left|\prod_{a=1}^{k} B_{\lambda_{a}, \mu_{a}}^{\dagger}\right| 0\right\rangle & =\prod_{a=1}^{k} \operatorname{Tr}\left(X^{\lambda_{a}} Y^{\mu_{a}}\right)
\end{aligned}
$$

In section 4.3 we will be studying in detail the transformation between the monomial multisymmetric functions and the trace wavefunctions. As a result of a triangular property of this transformation, the finite $N$ cutoff on multi-symmetric functions can also be described by restricting the number of factors in the product of traces to be less than or equal to $N$.

\subsection{Monomial and trace bases for multi-symmetric functions}

We now give a formal definition of multi-symmetric functions in a completely analogous way to the symmetric functions of section 2.1, just with two families of variables $x_{1}, \ldots, x_{N}$ and $y_{1}, \ldots, y_{N}$ instead of one. They are polynomials in these $2 N$ variables that are invariant under all $S_{N}$ permutations on the pairs $\left(x_{i}, y_{i}\right)$. Given a polynomial $f\left(x_{1}, x_{2}, \ldots, x_{N} ; y_{1}, y_{2}, \ldots, y_{N}\right), f$ is a multi-symmetric function if

$$
f\left(x_{1}, x_{2}, \ldots, x_{N} ; y_{1}, y_{2}, \ldots, y_{N}\right)=f\left(x_{\sigma(1)}, x_{\sigma(2)}, \ldots, x_{\sigma(N)} ; y_{\sigma(1)}, y_{\sigma(2)}, \ldots, y_{\sigma(N)}\right)
$$

for all $\sigma \in S_{N}$.

Similarly to section 2.1, we can take a large $N$ limit and work with formal power series rather than polynomials. We return to the finite $N$ case by setting $x_{N+1}=y_{N+1}=x_{N+2}=$ $y_{N+2}=\cdots=0$.

We can also define multi-symmetric functions with $M$ families of variables $x_{i}^{(k)}$, for $1 \leq k \leq M, 1 \leq i \leq N$, invariant under $S_{N}$ permutations of the $i$ index. These would be relevant for systems of $M$ commuting matrices.

For a mathematical overview of multi-symmetric functions and their properties see [56]. 
The monomial and multi-trace (power-sum) bases for symmetric polynomials defined in (2.10) and (2.12) have direct analogues in the multi-symmetric case. As before, they are graded bases, this time graded by both the $x$ degree $n_{1}$ and the $y$ degree $n_{2}$.

A vector partition $\mathbf{p}$ of $\left(n_{1}, n_{2}\right)$ is defined to be a sequence of pairs of non-negative integers (at least one of each pair must be non-zero) summing to $\left(n_{1}, n_{2}\right)$. We use a bold $\mathbf{p}$ to distinguish between vector and integer partitions, and write $\mathbf{p} \vdash\left(n_{1}, n_{2}\right)$ to denote that $\mathbf{p}$ sums to $\left(n_{1}, n_{2}\right)$. The basis elements at degree $\left(n_{1}, n_{2}\right)$ are labelled by $\mathbf{p} \vdash\left(n_{1}, n_{2}\right)$ with length $l(\mathbf{p}) \leq N$.

To construct the monomial basis, take a vector partition $\mathbf{p}=\left[\left(\lambda_{1}, \mu_{1}\right),\left(\lambda_{2}, \mu_{2}\right)\right.$, $\left.\ldots,\left(\lambda_{k}, \mu_{k}\right)\right]$ of $\left(n_{1}, n_{2}\right)$ with $l(\mathbf{p})=k \leq N$ and consider the un-symmetrised monomial

$$
x_{1}^{\lambda_{1}} y_{1}^{\mu_{1}} x_{2}^{\lambda_{2}} y_{2}^{\mu_{2}} \ldots x_{k}^{\lambda_{k}} y_{k}^{\mu_{k}}
$$

After adding all distinct permutations of the lower indices, one arrives at the monomial basis element. Explicitly

$$
m_{\mathbf{p}}=\frac{1}{Z_{\mathbf{p}}} \sum_{\sigma \in S_{N}} x_{\sigma(1)}^{\lambda_{1}} y_{\sigma(1)}^{\mu_{1}} x_{\sigma(2)}^{\lambda_{2}} y_{\sigma(2)}^{\mu_{2}} \ldots x_{\sigma(k)}^{\lambda_{k}} y_{\sigma(k)}^{\mu_{k}}
$$

where the factor in front removes the normalisation introduced by redundancies in the elements of $\mathbf{p}$, so that the coefficient in front of each individual monomial is 1 . Using multiplicity notation analogous to $(2.3)$, let $\mathbf{p}=<\mathbf{p}_{(0,1)}, \mathbf{p}_{(1,0)}, \cdots>$. Then the normalisation is given by

$$
Z_{\mathbf{p}}=\prod_{i, j} \mathbf{p}_{(i, j)} !
$$

As in the symmetric case, we will use a modified version of the monomial basis, obtained by leaving out this normalisation factor

$$
M_{\mathbf{p}}=Z_{\mathbf{p}} m_{\mathbf{p}}=\sum_{\sigma \in S_{N}} x_{\sigma(1)}^{\lambda_{1}} y_{\sigma(1)}^{\mu_{1}} x_{\sigma(2)}^{\lambda_{2}} y_{\sigma(2)}^{\mu_{2}} \ldots x_{\sigma(k)}^{\lambda_{k}} y_{\sigma(k)}^{\mu_{k}}
$$

As discussed below (4.16), we can lower $N$ to $N-1$ by setting $x_{N}=y_{N}=0$, causing a reduction in the size of the space. Starting from $N>n_{1}+n_{2}$ and reducing stepwise, this implies those monomial functions with $l(\mathbf{p})>N$ vanish identically, while the remaining $M_{\mathbf{p}}$ with $l(\mathbf{p}) \leq N$ form a basis for the smaller space. So the monomial basis is SEP-compatible for multi-symmetric functions.

Note that the isomorphism, as described around (3.7), states that multi-symmetric functions are isomorphic to invariants of matrices $X, Y$ modulo commutator traces. Therefore the isomorphic image of $M_{\mathbf{p}}$, also referred to as $M_{\mathbf{p}}$, is not necessarily zero if $l(\mathbf{p})>N$, but could instead be a commutator trace. This is the version of SEP-compatibility for preBPS operators as discussed in section 3.

As multi-symmetric functions, the monomial functions $M_{\mathbf{p}}$ are SEP-compatible. Using the map (3.6) to give the equivalent symmetrised trace operators, they form a basis for pre-BPS operators at $N \geq n$. As we decrease $N<n$, the SEP-compatibility implies any operator with $l(\mathbf{p})>N$ reduces to a commutator trace. However, the operators 
with $l(\mathbf{p}) \leq N$ are not in general $S_{n}$ orthogonal to commutator traces when $N<n$, and therefore they do not form a basis for pre-BPS operators. This is due to (3.6) not selecting the right representative of the equivalence class of operators isomorphic to the multi-symmetric function, as discussed below (3.7). We say the Yang-Mills operators $M_{\mathbf{p}}$ are SEP-compatible modulo commutators, and in section 6, describe how to transform this into a genuinely SEP-compatible basis for pre-BPS operators.

The multi-trace basis for multi-symmetric functions, also called the power-sum basis in the mathematics literature, is built out of

$$
T_{\left(n_{1}, n_{2}\right)}=\sum_{i=1}^{N} x_{i}^{n_{1}} y_{i}^{n_{2}}
$$

Given a vector partition $\mathbf{p}=\left[\left(\lambda_{1}, \mu_{1}\right),\left(\lambda_{2}, \mu_{2}\right), \ldots\left(\lambda_{k}, \mu_{k}\right)\right]$, the associated multi-symmetric function is

$$
T_{\mathbf{p}}=\prod_{i=1}^{k} T_{\left(\lambda_{i}, \mu_{i}\right)}
$$

Introduce two $N \times N$ diagonal matrices $X$ and $Y$ with diagonal elements $x_{1}, x_{2}, \ldots x_{N}$ and $y_{1}, y_{2}, \ldots y_{N}$ respectively. Then $T_{\left(n_{1}, n_{2}\right)}=\operatorname{Tr} X^{n_{1}} Y^{n_{2}}$, and the multi-trace multi-symmetric functions are exactly given by the multi-traces of these two matrices, justifying the name.

The isomorphism of $[35,36]$ identifies the multi-symmetric functions $(4.22)$ with the symmetrised trace operators (3.4), establishing the connection between multi-symmetric functions and the quarter-BPS sector of $\mathcal{N}=4$ super Yang-Mills at weak coupling.

Note that while (4.22) and (3.4) are conceptually different, the isomorphism between the two means we abuse notation slightly and use the same symbol $T_{\mathbf{p}}$ for both.

At finite $N$, non-trivial relationships appear between the different multi-traces leading to a reduction in the dimensionality of the space of multi-symmetric functions. Those multi-traces labelled by $\mathbf{p}$ with $l(\mathbf{p}) \leq N$ form a basis for the reduced space. However, unlike the monomial functions, the remaining multi-traces (labelled by $\mathbf{p}$ with $l(\mathbf{p})>N$ ) do not vanish, but become complicated linear combinations of the reduced basis.

Define a matrix $C_{\mathbf{q}}^{\mathbf{p}}$, indexed by vector partitions $\mathbf{p}$ and $\mathbf{q}$ to be the change of basis matrix from $M_{\mathbf{p}}$ to $T_{\mathbf{q}}$, with inverse $\widetilde{C}$

$$
T_{\mathbf{q}}=\sum_{\mathbf{p}} C_{\mathbf{q}}^{\mathbf{p}} M_{\mathbf{p}} \quad M_{\mathbf{p}}=\sum_{\mathbf{q}} \widetilde{C}_{\mathbf{p}}^{\mathbf{q}} T_{\mathbf{q}}
$$

At finite $N$, the $\mathbf{p}$ label for monomials is SEP-compatible (modulo commutators). Therefore the second of the equations above gives the finite $N$ relations imposed on commuting matrices. On the other side of the isomorphism, this gives the linear combinations of symmetrised traces that reduce to commutator traces at finite $N$.

The $C$ and $\widetilde{C}$ matrices have very interesting combinatorial properties, which we will now investigate in depth.

\subsection{Basis change for multi-symmetric functions}

In this section, we show that the properties of the linear transformations $C$ and $\widetilde{C}$ are illuminated by considering set partitions. Set partitions form a partially ordered set (poset), and the Möbius inversion formula for posets plays an important role. 
To find an expression for $C_{\mathbf{q}}^{\mathbf{p}}$, first expand the product in the definition (4.22) for multi-trace functions. For $\mathbf{p}=\left[\left(\lambda_{1}, \mu_{1}\right), \ldots,\left(\lambda_{k}, \mu_{k}\right)\right]$,

$$
\begin{aligned}
T_{\mathbf{p}} & =\prod_{j=1}^{k}\left(\sum_{i=1}^{N} x_{i}^{\lambda_{j}} y_{i}^{\mu_{j}}\right) \\
& =\sum_{j_{1}, \ldots, j_{k}=1}^{N} x_{j_{1}}^{\lambda_{1}} y_{j_{1}}^{\mu_{1}} x_{j_{2}}^{\lambda_{2}} y_{j_{2}}^{\mu_{2}} \ldots x_{j_{k}}^{\lambda_{k}} y_{j_{k}}^{\mu_{k}}
\end{aligned}
$$

To further sort this sum, note the different ways the $j$ s could coincide. If, for example, $k=3$, we could have

$$
\begin{array}{ll}
j_{1}=j_{2}=j_{3} & j_{1}=j_{2} \neq j_{3} \\
j_{1}=j_{3} \neq j_{2} & j_{1} \neq j_{2}=j_{3}
\end{array}
$$

$j_{1}, j_{2}, j_{3}$ all distinct

These correspond to the 5 different ways of partitioning the set $\{1,2,3\}$ into subsets

$$
\begin{array}{ll}
\pi_{1}=\{\{1,2,3\}\} & \pi_{2}=\{\{1,2\},\{3\}\} \\
\pi_{3}=\{\{1,3\},\{2\}\} & \pi_{4}=\{\{1\},\{2,3\}\} \\
\pi_{5}=\{\{1\},\{2\},\{3\}\} &
\end{array}
$$

Continuing with the example, we can sort the sum (4.25) into the different partitions

$$
\begin{aligned}
T_{\mathbf{p}}= & \sum_{j} x_{j}^{\lambda_{1}+\lambda_{2}+\lambda_{3}} y_{j}^{\mu_{1}+\mu_{2}+\mu_{3}}+\sum_{j_{1}, j_{3} \text { distinct }} x_{j_{1}}^{\lambda_{1}+\lambda_{2}} y_{j_{1}}^{\mu_{1}+\mu_{2}} x_{j_{3}}^{\lambda_{3}} y_{j_{3}}^{\mu_{3}} \\
& +\sum_{j_{1}, j_{2} \text { distinct }} x_{j_{1}}^{\lambda_{1}+\lambda_{3}} y_{j_{1}}^{\mu_{1}+\mu_{3}} x_{j_{2}}^{\lambda_{2}} y_{j_{2}}^{\mu_{2}}+\sum_{j_{1}, j_{2} \text { distinct }} x_{j_{1}}^{\lambda_{1}} y_{j_{1}}^{\mu_{1}} x_{j_{2}}^{\lambda_{2}+\lambda_{3}} y_{j_{2}}^{\mu_{2}+\mu_{3}} \\
& +\sum_{j_{1}, j_{2}, j_{3} \text { distinct }} x_{j_{1}}^{\lambda_{1}} y_{j_{1}}^{\mu_{1}} x_{j_{2}}^{\lambda_{2}} y_{j_{2}}^{\mu_{2}} x_{j_{3}}^{\lambda_{3}} y_{j_{3}}^{\mu_{3}}
\end{aligned}
$$

The first term is just the monomial function associated to the vector partition $\pi_{1}(\mathbf{p})=$ $\left[\left(\lambda_{1}+\lambda_{2}+\lambda_{3}, \mu_{1}+\mu_{2}+\mu_{3}\right)\right]$. Similarly, the second term is related to the monomial function with vector partition $\pi_{2}(\mathbf{p})=\left[\left(\lambda_{1}+\lambda_{2}, \mu_{1}+\mu_{2}\right),\left(\lambda_{3}, \mu_{3}\right)\right]$ via

$$
\sum_{j_{1}, j_{2} \text { distinct }} x_{j_{1}}^{\lambda_{1}+\lambda_{2}} y_{j_{1}}^{\mu_{1}+\mu_{2}} x_{j_{2}}^{\lambda_{3}} y_{j_{2}}^{\mu_{3}}= \begin{cases}2 m_{\pi_{2}(p)} & \left(\lambda_{1}+\lambda_{2}, \mu_{1}+\mu_{2}\right)=\left(\lambda_{3}, \mu_{3}\right) \\ m_{\pi_{2}(p)} & \text { otherwise }\end{cases}
$$

We can simplify this expression by noting that if $\left(\lambda_{1}+\lambda_{2}, \mu_{1}+\mu_{2}\right)=\left(\lambda_{3}, \mu_{3}\right)$ then $Z_{\pi_{2}(\mathbf{p})}=$ 2 , and otherwise $Z_{\pi_{2}(\mathbf{p})}=1$. Therefore

$$
\sum_{j_{1}, j_{2} \text { distinct }} x_{j_{1}}^{\lambda_{1}+\lambda_{2}} y_{j_{1}}^{\mu_{1}+\mu_{2}} x_{j_{2}}^{\lambda_{3}} y_{j_{2}}^{\mu_{3}}=Z_{\pi_{2}(\mathbf{p})} m_{\pi_{2}(\mathbf{p})}=M_{\pi_{2}(\mathbf{p})}
$$

Similarly the third, fourth and fifth terms of (4.32) are just $M_{\pi_{3}(\mathbf{p})}, M_{\pi_{4}(\mathbf{p})}$ and $M_{\pi_{5}(\mathbf{p})}$ respectively, where

$$
\begin{aligned}
& \pi_{3}(\mathbf{p})=\left[\left(\lambda_{1}+\lambda_{3}, \mu_{1}+\mu_{3}\right),\left(\lambda_{2}, \mu_{2}\right)\right] \\
& \pi_{4}(\mathbf{p})=\left[\left(\lambda_{1}, \mu_{1}\right),\left(\lambda_{2}+\lambda_{3}, \mu_{2}+\mu_{3}\right)\right] \\
& \pi_{5}(\mathbf{p})=\mathbf{p}
\end{aligned}
$$


Putting this together, we have

$$
T_{\mathbf{p}}=\sum_{i=1}^{5} M_{\pi_{i}(\mathbf{p})}
$$

Repeating this analysis more generally, let the set of set partitions of $\{1,2,3, \ldots k\}$ be denoted by $\Pi(k)$. Then given a set partition $\pi \in \Pi(k)$ and a vector partition $\mathbf{p}=$ $\left[\left(\lambda_{1}, \mu_{1}\right), \ldots,\left(\lambda_{k}, \mu_{k}\right)\right]$ of length $l(\mathbf{p})=k$, we define the vector partition $\pi(k)$ to be that with components

$$
\left(\sum_{i \in b} \lambda_{i}, \quad \sum_{i \in b} \mu_{i}\right)
$$

where the blocks $b \in \pi$ run over the subsets into which $\{1,2,3, \ldots k\}$ have been partitioned. Conceptually, this should be thought of as summing up $\mathbf{p}$ into a new, shorter vector partition, where the summation structure is given by $\pi$.

Given this notation, we can now write the generalisation of (4.38) to any $k$

$$
T_{\mathbf{p}}=\sum_{\pi \in \Pi(l(\mathbf{p}))} M_{\pi(\mathbf{p})}
$$

Proving this result in the general case is just an exercise in repeating the logic that led from (4.25) to (4.38).

So the coefficient of $M_{\mathbf{p}}$ in $T_{\mathbf{q}}$ is just the number of set partitions $\pi \in \Pi(l(\mathbf{q}))$ that have $\pi(\mathbf{q})=\mathbf{p}$.

$$
C_{\mathbf{q}}^{\mathbf{p}}=\sum_{\pi \in \Pi(l(\mathbf{q}))} \delta_{\mathbf{p} \pi(\mathbf{q})}
$$

We can see that for vector partitions of a particular length $k=l(\mathbf{q})$, it is the set partitions of $\{1,2,3, \ldots, k\}$ that control the behaviour.

The poset (partially ordered set) structure of set of set partitions is well studied [57], and will help further explain the structure of the matrix $C$ and its inverse $\widetilde{C}$. The partial ordering is defined by saying that one set partition, $\pi$, is less than another, $\pi^{\prime}$, if every block $b \in \pi$ is contained within some block $b^{\prime} \in \pi^{\prime}$. We call $\pi$ a refinement of $\pi^{\prime}$ or $\pi^{\prime}$ a coarsening of $\pi$.

Intuitively, if $\pi<\pi^{\prime}$, then the blocks of $\pi$ are smaller in size than those in $\pi^{\prime}$. However, this means that there are more blocks in $\pi$ than in $\pi^{\prime}$, so confusingly $\pi<\pi^{\prime}$ implies that $|\pi|>\left|\pi^{\prime}\right|$.

Now instead of looking at $T_{\mathbf{p}}$, we look at $T_{\pi(\mathbf{p})}$, for some $\pi \in \Pi(l(\mathbf{p}))$. Clearly we can still use the formula (4.40) just by replacing $\mathbf{p}$ with $\pi(\mathbf{p})$. Then summing over $\pi^{\prime} \in$ $\Pi(l(\pi(\mathbf{p})))$ with summand $M_{\pi^{\prime}(\pi(\mathbf{p}))}$ is equivalent to summing over all coarsenings $\pi^{\prime \prime} \geq \pi$ with summand $M_{\pi^{\prime \prime}(\mathbf{p})}$, so we can write

$$
T_{\pi(\mathbf{p})}=\sum_{\pi^{\prime} \geq \pi} M_{\pi^{\prime}(\mathbf{p})}
$$

Considering $T_{\pi}$ and $M_{\pi}$ as functions from vector partitions to multi-symmetric functions, we have

$$
T_{\pi}=\sum_{\pi^{\prime} \geq \pi} M_{\pi^{\prime}}
$$


Equations like (4.43) are standard the theory of posets [57], and can be inverted using the Möbius inversion formula (4.49). We explain this formula in more detai 1 in section 4.4, including a combinatoric interpretation that allows a simple explanation of the inversion property.

In this case, the Möbius inversion formula implies

$$
M_{\pi}=\sum_{\pi^{\prime} \geq \pi} \mu\left(\pi, \pi^{\prime}\right) T_{\pi^{\prime}}
$$

where the Möbius function $\mu\left(\pi, \pi^{\prime}\right)$ is defined in (4.53).

Choosing a vector partition $\mathbf{p}$ on which to act, we have

$$
M_{\pi(\mathbf{p})}=\sum_{\pi^{\prime} \geq \pi} \mu\left(\pi, \pi^{\prime}\right) T_{\pi^{\prime}(\mathbf{p})}
$$

We can now use this to find an explicit expression for $\widetilde{C}_{\mathbf{p}}^{\mathbf{q}}$. Let $k=l(\mathbf{p})$ and $\pi=\pi_{k}$ to be the minimal set partition in $\Pi(k)$, in which every element has its own block so that $\pi_{k}(\mathbf{p})=\mathbf{p}$. Applying (4.45) gives

$$
M_{\mathbf{p}}=\sum_{\pi \in \Pi(k)} \mu\left(\pi_{k}, \pi\right) T_{\pi(\mathbf{p})}
$$

and therefore

$$
\widetilde{C}_{\mathbf{q}}^{\mathbf{p}}=\sum_{\pi \in \Pi(k)} \mu\left(\pi_{k}, \pi\right) \delta_{\mathbf{p} \pi(\mathbf{q})}
$$

\subsection{Möbius function for the poset of set partitions and combinatoric interpre- tation}

In this section we introduce the Möbius function for a general poset, and give its value on the poset of set partitions. There is a combinatoric interpretation for the Möbius function in terms of permutations on the blocks of the set partitions, and this interpretation allows us to simply see why the Möbius inversion formula works in this case.

The Möbius function is defined recursively for a generic poset by

$$
\mu\left(\pi, \pi^{\prime}\right)= \begin{cases}1 & \pi=\pi^{\prime} \\ -\sum_{\pi \leq \pi^{\prime \prime}<\pi^{\prime}} \mu\left(\pi, \pi^{\prime \prime}\right) & \pi<\pi^{\prime} \\ 0 & \text { otherwise }\end{cases}
$$

The key utility of this definition is in the Möbius inversion formula, which states that given two functions $f, g$ from a poset into a vector space, the following two relations are equivalent

$$
\begin{aligned}
& f(\pi)=\sum_{\pi^{\prime} \geq \pi} g\left(\pi^{\prime}\right) \\
& g(\pi)=\sum_{\pi^{\prime} \geq \pi} \mu\left(\pi, \pi^{\prime}\right) f\left(\pi^{\prime}\right)
\end{aligned}
$$


In order to give an explicit expression for the Möbius function on set partitions, consider $\pi=\left\{b_{1}, b_{2}, \ldots, b_{k}\right\}$ for $k=|\pi|$. We then look at the set partitions of $\pi$ itself. For example if $k=3$ the five possible set partitions of $\pi$ are

$$
\begin{array}{ll}
\rho_{1}=\left\{\left\{b_{1}, b_{2}, b_{3}\right\}\right\} & \rho_{2}=\left\{\left\{b_{1}, b_{2}\right\},\left\{b_{3}\right\}\right\} \\
\rho_{3}=\left\{\left\{b_{1}, b_{3}\right\},\left\{b_{2}\right\}\right\} & \rho_{4}=\left\{\left\{b_{1}\right\},\left\{b_{2}, b_{3}\right\}\right\} \\
\rho_{5}=\left\{\left\{b_{1}\right\},\left\{b_{2}\right\},\left\{b_{3}\right\}\right\} &
\end{array}
$$

The set of set partitions of $\pi$ is denoted by $\Pi(\pi)$, and there is an obvious correspondence between this and $\Pi(|\pi|)$. For any particular $\pi \in \Pi(n)$ and $\rho \in \Pi(\pi)$, we define $\rho(\pi)$ to be the following set partition in $\Pi(n)$.

$$
\left\{\bigcup_{b \in B} b: B \in \rho\right\}
$$

So for the examples in (4.50), we have

$$
\begin{array}{ll}
\rho_{1}(\pi)=\left\{b_{1} \cup b_{2} \cup b_{3}\right\} & \rho_{2}(\pi)=\left\{b_{1} \cup b_{2}, b_{3}\right\} \\
\rho_{3}(\pi)=\left\{b_{1} \cup b_{3}, b_{2}\right\} & \rho_{4}(\pi)=\left\{b_{1}, b_{2} \cup b_{3}\right\} \\
\rho_{5}(\pi)=\left\{b_{1}, b_{2}, b_{3}\right\}=\pi &
\end{array}
$$

Given $\pi \leq \pi^{\prime}$, by definition each block $b \in \pi$ is a subset of a block $b^{\prime} \in \pi^{\prime}$. Therefore there is a set partition $\rho \in \Pi(\pi)$ such that $\rho(\pi)=\pi^{\prime}$, we call this set partition $\pi^{\prime} / \pi$.

Using the definition of $\pi^{\prime} / \pi$ for $\pi^{\prime} \geq \pi$, we can now give an expression for $\mu\left(\pi, \pi^{\prime}\right)$, which is a standard result in the field of posets [57]. Firstly, by definition $\mu\left(\pi, \pi^{\prime}\right)$ vanishes unless $\pi^{\prime}<\pi$, so we assume $\pi^{\prime} \geq \pi$. This means $\pi^{\prime} / \pi$ exists, and we can write

$$
\mu\left(\pi, \pi^{\prime}\right)=(-1)^{|\pi|-\left|\pi^{\prime}\right|} \prod_{b \in \pi^{\prime} / \pi}(|b|-1) !
$$

There is a combinatoric interpretation for the magnitude of $\mu\left(\pi, \pi^{\prime}\right)$ in terms of permutations, where the sign of $\mu$ is given by the sign of these permutations. In order to describe this, consider a permutation $\sigma \in S_{n}$ and take an arbitrary subset $A \subseteq\{1,2, \ldots, n\}$. Then $\sigma$ acts on $A$ by permuting the numbers 1 to $n$, leading to a distinct subset $\sigma(A)$. We can then define the subgroup $G(\pi) \leq S_{n}$ by

$$
G(\pi)=\{\sigma: \sigma(b)=b \text { for all blocks } b \in \pi\}
$$

For $\pi$ with block sizes of $\left[\lambda_{1}, \lambda_{2}, \ldots, \lambda_{k}\right] \vdash n$, we have

$$
G(\pi) \cong S_{\lambda_{1}} \times S_{\lambda_{2}} \times \cdots \times S_{\lambda_{k}}
$$

Intuitively, the $S_{\lambda_{i}}$ factor permutes the elements of the corresponding block with size $\lambda_{i}$. The exact embedding of $S_{\lambda_{1}} \times \cdots \times S_{\lambda_{k}}$ into $S_{n}$ depends on the set partition. 
Take a $\sigma \in S_{n}$. The cycle structure of $\sigma$ defines a partition $\pi(\sigma) \in \Pi(n)$. Formally, the set partition $\pi(\sigma)$ is simply the set of orbits of $\{1,2, \ldots, n\}$ under the action of $\sigma$. We also define a set of permutations associated with each $\pi \in \Pi(n)$

$$
\operatorname{Perms}(\pi)=\{\sigma: \pi(\sigma)=\pi\}
$$

For any $\sigma \in S_{n}$ with $\pi(\sigma)=\pi$, Perms $(\pi)$ is just the conjugacy class of $\sigma$ under conjugation by $G(\pi)$.

Clearly $\operatorname{Perms}(\pi)$ are disjoint for different $\pi$, and between them they cover $S_{n}$.

$$
\bigsqcup_{\pi \in \Pi(n)} \operatorname{Perms}(\pi)=S_{n}
$$

We have a similar result for $G(\pi)$, obtained by taking the decomposition (4.55) and applying (4.57) to each factor individually.

$$
\bigsqcup_{\pi^{\prime} \leq \pi} \operatorname{Perms}\left(\pi^{\prime}\right)=G(\pi)
$$

To illustrate the above, we now give some examples. If we fix $\pi=\{\{1,2,3\},\{4,5\},\{6\}\}$ then

$$
\begin{aligned}
G(\pi) & =S_{\{1,2,3\}} \times S_{\{4,5\}} \times S_{\{6\}} \cong S_{3} \times S_{2} \times S_{1} \\
\operatorname{Perms}(\pi) & =\{(1,2,3)(4,5),(1,3,2)(4,5)\}
\end{aligned}
$$

Enumerating the elements of $G(\pi)$, we can see that it splits as specified in (4.58).

$$
\begin{aligned}
G(\pi)=\{e & ,(1,2),(1,3),(2,3),(1,2,3),(1,3,2), \\
& (4,5),(1,2)(4,5),(1,3)(4,5),(2,3)(4,5),(1,2,3)(4,5),(1,3,2)(4,5)\} \\
=\{e\} & \sqcup\{(1,2)\} \sqcup\{(1,3)\} \sqcup\{(2,3)\} \sqcup\{(1,2,3),(1,3,2)\} \\
& \sqcup\{(4,5)\} \sqcup\{(1,2)(4,5)\} \sqcup\{(1,3)(4,5)\} \sqcup\{(2,3)(4,5)\} \\
& \sqcup\{(1,2,3)(4,5),(1,3,2)(4,5)\} \\
= & \operatorname{Perms}(\{\{1\},\{2\},\{3\},\{4\},\{5\},\{6\}\}) \sqcup \operatorname{Perms}(\{\{1,2\},\{3\},\{4\},\{5\},\{6\}\}) \\
& \sqcup \operatorname{Perms}(\{\{1,3\},\{2\},\{4\},\{5\},\{6\}\}) \sqcup \operatorname{Perms}(\{\{1\},\{2,3\},\{4\},\{5\},\{6\}\}) \\
& \sqcup \operatorname{Perms}(\{\{1,2,3\},\{4\},\{5\},\{6\}\}) \sqcup \operatorname{Perms}(\{\{1\},\{2\},\{3\},\{4,5\},\{6\}\}) \\
& \sqcup \operatorname{Perms}(\{\{1,2\},\{3\},\{4,5\},\{6\}\}) \sqcup \operatorname{Perms}(\{\{1,3\},\{2\},\{4,5\},\{6\}\}) \\
& \sqcup \operatorname{Perms}(\{\{1\},\{2,3\},\{4,5\},\{6\}\}) \sqcup \operatorname{Perms}(\{\{1,2,3\},\{4,5\},\{6\}\}) \\
= & \sqcup \operatorname{Perms}\left(\pi^{\prime}\right)
\end{aligned}
$$

Equations (4.54)-(4.62) are based on using permutations $\sigma \in S_{n}$ and set partitions $\pi \in$ $\Pi(n)$. However, if we pick $\pi \in \Pi(n)$, we can use the exact same constructions for permutations of $\pi$ itself — we call this group $S_{\pi}$ - and set partitions $\rho \in \Pi(\pi)$. Then $\operatorname{Perms}\left(\pi^{\prime} / \pi\right)$ provides our combinatoric interpretation for $\mu$

$$
\mu\left(\pi, \pi^{\prime}\right)=\sum_{\sigma \in \operatorname{Perms}\left(\pi^{\prime} / \pi\right)} \operatorname{sgn}(\sigma)=(-1)^{|\pi|-\left|\pi^{\prime}\right|}\left|\operatorname{Perms}\left(\pi^{\prime} / \pi\right)\right|
$$


So the magnitude of $\mu$ is just the number of permutations in a certain conjugacy class, and its sign is just the sign of these permutations.

This permutation interpretation of $\mu$ allows us to easily prove the Möbius inversion formula for set partitions. Fix $\pi$ and $\pi^{\prime \prime}$ with $\pi^{\prime \prime} \geq \pi$ and consider the sum

$$
\sum_{\pi^{\prime \prime} \geq \pi^{\prime} \geq \pi} \mu\left(\pi, \pi^{\prime}\right)
$$

The simplest way to parameterise the sum over $\pi^{\prime}$ is to look at the possible $\pi^{\prime} / \pi \in \Pi(\pi)$. The condition $\pi^{\prime \prime} \geq \pi^{\prime}$ becomes $\left(\pi^{\prime \prime} / \pi\right) \geq\left(\pi^{\prime} / \pi\right)$, so instead of summing over $\pi^{\prime} \in \Pi(n)$, we sum over $\pi^{\prime} / \pi=\rho \in \Pi(\pi)$.

$$
\begin{aligned}
\sum_{\pi^{\prime \prime} \geq \pi^{\prime} \geq \pi} \mu\left(\pi, \pi^{\prime}\right) & =\sum_{\rho \leq\left(\pi^{\prime \prime} / \pi\right)} \sum_{\sigma \in \operatorname{Perms}(\rho)} \operatorname{sgn}(\sigma) \\
& =\sum_{\sigma \in G\left(\pi^{\prime \prime} / \pi\right)} \operatorname{sgn}(\sigma) \\
& = \begin{cases}1 & G\left(\pi^{\prime \prime} / \pi\right) \cong S_{1} \times S_{1} \times \cdots \times S_{1} \\
0 & \text { otherwise }\end{cases}
\end{aligned}
$$

Where we have used (4.58) to change the sum into one over $G\left(\pi^{\prime \prime} / \pi\right)$, and the final line is a simple fact from permutation group theory. Now the only case for which $G\left(\pi^{\prime \prime} / \pi\right) \cong$ $S_{1} \times \cdots \times S_{1}$ is when $\pi^{\prime \prime}=\pi$, so we conclude that

$$
\sum_{\pi^{\prime \prime} \geq \pi^{\prime} \geq \pi} \mu\left(\pi, \pi^{\prime}\right)=\delta_{\pi \pi^{\prime \prime}}
$$

Using this result and substituting (4.43), we have

$$
\begin{aligned}
\sum_{\pi^{\prime} \geq \pi} \mu\left(\pi, \pi^{\prime}\right) T_{\pi^{\prime}} & =\sum_{\pi^{\prime \prime} \geq \pi^{\prime} \geq \pi} \mu\left(\pi, \pi^{\prime}\right) M_{\pi^{\prime \prime}} \\
& =M_{\pi}
\end{aligned}
$$

This proves the Möbius inversion formula for set partitions.

For a more thorough overview of the Möbius function on general posets and for the poset of set partitions see [57].

\subsection{More general $C$ and $\widetilde{C}$ matrices for $M$-matrix systems}

The structures explained in sections 4.3 and 4.4, involving the poset of set partitions and the associated Möbius function, can be used not just for the two matrix system, but for an $M$-matrix system. In (4.43) we introduced $T_{\pi}$ and $M_{\pi}$ as functions from 2-vector partitions of length $|\pi|$ into the space of multi-symmetric functions defined on two families of variables, $x_{i}$ and $y_{i}$. However, we could equally consider them as functions from $M$ vector partitions of length $|\pi|$ into the space of multi-symmetric functions defined on $M$ families of variables, $x_{i}^{(1)}, x_{i}^{(2)}, \ldots, x_{i}^{(M)}$. These multi-symmetric functions have monomial and multi-trace bases defined in direct analogy to the 2-vector versions in section 4.2. For 
each $M$ there are corresponding $C$ and $\widetilde{C}$ matrices, defined in a completely analogous way to (4.41) and (4.47).

To think about these possibilities in a unified way, we define a more general $C$ and $\widetilde{C}$ that transform between $T_{\pi}$ and $M_{\pi}$.

$$
T_{\pi}=\sum_{\pi^{\prime}} C_{\pi}^{\pi^{\prime}} M_{\pi^{\prime}} \quad M_{\pi}=\sum_{\pi^{\prime}} \widetilde{C}_{\pi}^{\pi^{\prime}} T_{\pi^{\prime}}
$$

We already have expressions for these from (4.43) and (4.44), given by

$$
C_{\pi}^{\pi^{\prime}}=\zeta\left(\pi, \pi^{\prime}\right)=\left\{\begin{array}{ll}
1 & \pi^{\prime} \geq \pi \\
0 & \text { otherwise }
\end{array} \quad \widetilde{C}_{\pi}^{\pi^{\prime}}=\mu\left(\pi, \pi^{\prime}\right)\right.
$$

where the first equation defines $\zeta\left(\pi, \pi^{\prime}\right)$. The above also serves as the definition for the $\zeta$ function of a general poset, just with $\pi, \pi^{\prime}$ arbitrary elements of the poset rather than set partitions. We have already seen the Möbius function for a general poset in (4.48). An equivalent way of stating the Möbius inversion formula seen in (4.49) is that the $\zeta$ and $\mu$ are inverses of each other when multiplied as matrices

$$
\sum_{\pi^{\prime}} \zeta\left(\pi, \pi^{\prime}\right) \mu\left(\pi^{\prime}, \pi^{\prime \prime}\right)=\delta_{\pi \pi^{\prime \prime}}
$$

The $C$ and $\widetilde{C}$ for vector partitions (both 2 -vectors and $M$-vectors) can easily be obtained from these more general objects. For $\mathbf{p}, \mathbf{q} \vdash\left(n_{1}, n_{2}\right)$ and some $\pi \in \Pi(n)$ such that $\pi\left(\left[(1,0)^{n_{1}},(0,1)^{n_{2}}\right]\right)=\mathbf{p}$, we have

$$
C_{\mathbf{p}}^{\mathbf{q}}=\sum_{\pi^{\prime} \in \Pi(n)} C_{\pi}^{\pi^{\prime}} \quad \widetilde{C}_{\mathbf{p}}^{\mathbf{q}}=\sum_{\pi^{\prime} \in \Pi(n)} \widetilde{C}_{\pi}^{\pi^{\prime}}
$$

where the sums run over $\pi^{\prime}$ with $\pi^{\prime}\left(\left[(1,0)^{n_{1}},(0,1)^{n_{2}}\right]\right)=\mathbf{q}$. Analogous formulae hold for $M$-vectors.

We can think of the (4.71) as a flavour projection from the general system of $M_{\pi}, T_{\pi}$ to the $M$-flavour system consisting of $M_{\mathbf{p}}$ and $T_{\mathbf{p}}$. Physically, one flavour corresponds to the half-BPS sector, two flavours to the quarter-BPS sector, and three to the eighth-BPS sector. We give an alternative viewpoint on the flavour projection using permutations in section 7.1.2.

As an example, consider $n=4$. There are 15 different set partitions in $\Pi(4)$, so to simplify things we only give the transformations for the five different orbits under $S_{4}$, corresponding to the integer partitions of 4 . The $C$ matrix can be read off from the relationships showing $T_{\pi}$ in terms of $M_{\pi}$.

$$
\begin{aligned}
T_{\{\{1,2,3,4\}\}}= & M_{\{\{1,2,3,4\}\}} \\
T_{\{\{1\},\{2,3,4\}\}}= & M_{\{\{1\},\{2,3,4\}\}}+M_{\{\{1,2,3,4\}\}} \\
T_{\{\{1,2\},\{3,4\}\}}= & M_{\{\{1,2\},\{3,4\}\}}+M_{\{\{1,2,3,4\}\}} \\
T_{\{\{1,2\},\{3\},\{4\}\}}= & M_{\{\{1,2\},\{3\},\{4\}\}}+M_{\{\{1,2,3\},\{4\}\}}+M_{\{\{1,2,4\},\{3\}\}} \\
& +M_{\{\{1,2\},\{3,4\}\}}+M_{\{\{1,2,3,4\}\}}
\end{aligned}
$$




$$
\begin{aligned}
T_{\{\{1\},\{2\},\{3\},\{4\}\}}= & M_{\{\{1\},\{2\},\{3\},\{4\}\}}+M_{\{\{1,2\},\{3\},\{4\}\}}+M_{\{\{1,3\},\{2\},\{4\}\}} \\
& +M_{\{\{1,4\},\{2\},\{3\}\}}+M_{\{\{1\},\{2,3\},\{4\}\}}+M_{\{\{1\},\{2,4\},\{3\}\}} \\
& +M_{\{\{1\},\{2\},\{3,4\}\}}+M_{\{\{1,2\},\{3,4\}\}}+M_{\{\{1,3\},\{2,4\}\}} \\
& +M_{\{\{1,4\},\{2,3\}\}}+M_{\{\{1,2,3\},\{4\}\}}+M_{\{\{1,2,4\},\{3\}\}} \\
& +M_{\{\{1,3,4\},\{2\}\}}+M_{\{\{1\},\{2,3,4\}\}}+M_{\{11,2,3,4\}\}}
\end{aligned}
$$

The $\widetilde{C}$ matrix can be shown in an analogous way by writing $M_{\pi}$ in terms of $T_{\pi}$.

$$
\begin{aligned}
M_{\{\{1,2,3,4\}\}}= & T_{\{\{1,2,3,4\}\}} \\
M_{\{\{1\},\{2,3,4\}\}}= & T_{\{\{1\},\{2,3,4\}\}}-T_{\{\{1,2,3,4\}\}} \\
M_{\{\{1,2\},\{3,4\}\}}= & T_{\{\{1,2\},\{3,4\}\}}-T_{\{\{1,2,3,4\}\}} \\
M_{\{\{1,2\},\{3\},\{4\}\}}= & T_{\{\{1,2\},\{3\},\{4\}\}}-T_{\{\{1,2,3\},\{4\}\}}-T_{\{\{1,2,4\},\{3\}\}} \\
& -T_{\{\{1,2\},\{3,4\}\}}+2 T_{\{\{1,2,3,4\}\}} \\
M_{\{\{1\},\{2\},\{3\},\{4\}\}}= & T_{\{\{1\},\{2\},\{3\},\{4\}\}}-T_{\{\{1,2\},\{3\},\{4\}\}}-T_{\{\{1,3\},\{2\},\{4\}\}} \\
& -T_{\{\{1,4\},\{2\},\{3\}\}}-T_{\{\{1\},\{2,3\},\{4\}\}}-T_{\{\{1\},\{2,4\},\{3\}\}} \\
& -T_{\{\{1\},\{2\},\{3,4\}\}}+T_{\{\{1,2\},\{3,4\}\}}+T_{\{\{1,3\},\{2,4\}\}} \\
& +T_{\{\{1,4\},\{2,3\}\}}+2 T_{\{\{1,2,3\},\{4\}\}}+2 T_{\{\{1,2,4\},\{3\}\}} \\
& +2 T_{\{\{1,3,4\},\{2\}\}}+2 T_{\{\{1\},\{2,3,4\}\}}-6 T_{\{\{1,2,3,4\}\}}
\end{aligned}
$$

We can then apply these to the vector partition $\left[(1,0)^{2},(0,1)^{2}\right]$ to get $C$ and $\widetilde{C}$ for field content $(2,2)$. Again we choose to display them by writing out the relations between $T_{\mathbf{p}}$ and $M_{\mathbf{p}}$, but this time all possibilities are included. The $C$ matrix is

$$
\begin{aligned}
\operatorname{Str} X^{2} Y^{2}= & M_{[(2,2)]} \\
\operatorname{Tr} X^{2} Y \operatorname{Tr} Y= & M_{[(2,1),(0,1)]}+M_{[(2,2)]} \\
\operatorname{Tr} X^{2} \operatorname{Tr} Y^{2}= & M_{[(2,0),(0,2)]}+M_{[(2,2)]} \\
\operatorname{Tr} X Y^{2} \operatorname{Tr} X= & M_{[(1,2),(1,0)]}+M_{[(2,2)]} \\
(\operatorname{Tr} X Y)^{2}= & M_{[(1,1),(1,1)]}+M_{[(2,2)]} \\
\operatorname{Tr} X^{2}(\operatorname{Tr} Y)^{2}= & M_{[(2,0),(0,1),(0,1)]}+M_{[(2,0),(0,2)]}+2 M_{[(2,1),(0,1)]}+M_{[(2,2)]} \\
\operatorname{Tr} X Y \operatorname{Tr} X \operatorname{Tr} Y= & M_{[(1,1),(1,0),(0,1)]}+M_{[(1,1),(1,1)]}+M_{[(1,2),(1,0)]} \\
& +M_{[(2,1),(0,1)]}+M_{[(2,2)]} \\
(\operatorname{Tr} X)^{2} \operatorname{Tr} Y^{2}= & M_{[(1,0),(1,0),(0,2)]}+2 M_{[(1,2),(1,0)]}+M_{[(2,0),(0,2)]}+M_{[(2,2)]} \\
(\operatorname{Tr} X)^{2}(\operatorname{Tr} Y)^{2}= & M_{[(1,0),(1,0),(0,1),(0,1)]}+M_{[(1,0),(1,0),(0,2)]}+4 M_{[(1,1),(1,0),(0,1)]} \\
& +M_{[(2,0),(0,1),(0,1)]}+2 M_{[(1,1),(1,1)]}+2 M_{[(1,2),(1,0)]} \\
& +M_{[(2,0),(0,2)]}+2 M_{[(2,1),(0,1)]}+M_{[(2,2)]}
\end{aligned}
$$

The $\widetilde{C}$ matrix for $(2,2)$ is

$$
\begin{aligned}
M_{[(2,2)]} & =\operatorname{Str} X^{2} Y^{2} \\
M_{[(2,1),(0,1)]} & =\operatorname{Tr} X^{2} Y \operatorname{Tr} Y-\operatorname{Str} X^{2} Y^{2}
\end{aligned}
$$




$$
\begin{aligned}
M_{[(2,0),(0,2)]}= & \operatorname{Tr} X^{2} \operatorname{Tr} Y^{2}-\operatorname{Str} X^{2} Y^{2} \\
M_{[(1,2),(1,0)]}= & \operatorname{Tr} X Y^{2} \operatorname{Tr} X-\operatorname{Str} X^{2} Y^{2} \\
M_{[(1,1),(1,1)]}= & (\operatorname{Tr} X Y)^{2}-\operatorname{Str} X^{2} Y^{2} \\
M_{[(2,0),(0,1),(0,1)]}= & \operatorname{Tr} X^{2}(\operatorname{Tr} Y)^{2}-\operatorname{Tr} X^{2} \operatorname{Tr} Y^{2}-2 \operatorname{Tr} X^{2} Y \operatorname{Tr} Y+2 \operatorname{Str} X^{2} Y^{2} \\
M_{[(1,1),(1,0),(0,1)]}= & \operatorname{Tr} X Y \operatorname{Tr} X \operatorname{Tr} Y-(\operatorname{Tr} X Y)^{2}-\operatorname{Tr} X Y^{2} \operatorname{Tr} X \\
& -\operatorname{Tr} X^{2} Y \operatorname{Tr} Y+2 \operatorname{Str} X^{2} Y^{2} \\
M_{[(1,0),(1,0),(0,2)]}= & (\operatorname{Tr} X)^{2} \operatorname{Tr} Y^{2}-2 \operatorname{Tr} X Y^{2} \operatorname{Tr} X-\operatorname{Tr} X^{2} \operatorname{Tr} Y^{2}+2 \operatorname{Str} X^{2} Y^{2} \\
M_{[(1,0),(1,0),(0,1),(0,1)]}= & (\operatorname{Tr} X)^{2}(\operatorname{Tr} Y)^{2}-(\operatorname{Tr} X)^{2} \operatorname{Tr} Y^{2}-4 \operatorname{Tr} X Y \operatorname{Tr} X \operatorname{Tr} Y \\
& -\operatorname{Tr} X^{2}(\operatorname{Tr} Y)^{2}+2(\operatorname{Tr} X Y)^{2}+4 \operatorname{Tr} X Y^{2} \operatorname{Tr} X \\
& +\operatorname{Tr} X^{2} \operatorname{Tr} Y^{2}+4 \operatorname{Tr} X^{2} Y \operatorname{Tr} Y-6 \operatorname{Str} X^{2} Y^{2}
\end{aligned}
$$

Note that we have used

$$
\operatorname{Str} X^{2} Y^{2}=\frac{2}{3} \operatorname{Tr} X^{2} Y^{2}+\frac{1}{3} \operatorname{Tr}(X Y)^{2}
$$

rather than just $\operatorname{Tr} X^{2} Y^{2}$. This means the expressions (4.82)-(4.99) give the relations between $T_{\mathbf{p}}$ and $M_{\mathbf{p}}$ both as multi-symmetric functions and symmetrised trace operators in $\mathcal{N}=4$ super Yang-Mills.

\subsection{Relation to other combinatorial quantities}

Stirling numbers of the second kind, $S(n, k)$, are defined to be the number of ways of partitioning a set of $n$ objects into $k$ non-empty subsets. Combinatorically, these are a coarsened version of the 2 -vector and set partition $C$ matrices. Starting with the 2-vector version, $S(n, k)$ is given by

$$
S(n, k)=\sum_{\substack{\mathbf{p} \vdash-\left(n_{1}, n_{2}\right) \\ l(\mathbf{p})=k}} C_{\left[(1,0)^{n_{1}},(0,1)^{n_{2}}\right]}^{\mathbf{p}}
$$

where $n_{1}+n_{2}=n$.

Alternatively, consider an arbitrary $m>n$ and $\mathbf{q} \vdash\left(m_{1}, m_{2}\right)$ with $m_{1}+m_{2}=m$ to be a vector partition with $l(\mathbf{q})=n$, then

$$
S(n, k)=\sum_{\substack{\mathbf{p} \vdash\left(m_{1}, m_{2}\right) \\ l(\mathbf{p})=k}} C_{\mathbf{q}}^{\mathbf{p}}
$$

Define $\pi_{n} \in \Pi(n)$ to be the unique set partition of length $n$, meaning each number has its own block. Then in terms of the more general set partition $C$

$$
S(n, k)=\sum_{\substack{\pi \in \Pi(n) \\|\pi|=k}} C_{\pi_{n}}^{\pi}
$$

Or alternatively, taking $\pi \in \Pi(m)$ to be any set partition with $|\pi|=n$, then

$$
S(n, k)=\sum_{\substack{\pi^{\prime} \in \Pi(m) \\\left|\pi^{\prime}\right|=k}} C_{\pi}^{\pi^{\prime}}
$$


Unsigned Stirling numbers of the first kind, $|s(n, k)|$, are defined to be the number of permutations in $S_{n}$ with $k$ cycles. The signed Stirling numbers $s(n, k)$ have the same magnitude, but are multiplied by the sign of the permutations $(-1)^{n-k}$. This is related to the 2-vector and set partition $\widetilde{C}$ matrices in the same way as $S(n, k)$ was related to $C$. Using the same notation as (4.101)-(4.104), we have

$$
\begin{array}{ll}
s(n, k)=\sum_{\substack{\mathbf{p} \vdash\left(n_{1}, n_{2}\right) \\
l(\mathbf{p})=k}} \widetilde{C}_{\left[(1,0)^{n_{1}},(0,1)^{n_{2}}\right]}^{\mathbf{p}} & s(n, k)=\sum_{\substack{\mathbf{p} \vdash\left(m_{1}, m_{2}\right) \\
l(\mathbf{p})=k}} \widetilde{C}_{\mathbf{q}}^{\mathbf{p}} \\
s(n, k)=\sum_{\substack{\pi \in \Pi(n) \\
|\pi|=k}} \widetilde{C}_{\pi_{n}}^{\pi} & s(n, k)=\sum_{\substack{\pi^{\prime} \in \Pi(m) \\
\left|\pi^{\prime}\right|=k}} \widetilde{C}_{\pi}^{\pi^{\prime}}
\end{array}
$$

Bell numbers, $B_{n}$, count the number of set partitions of $n$ objects. In terms of $C$, these are

$$
B_{n}=\sum_{k} S(n, k)=\sum_{\mathbf{p} \vdash\left(n_{1}, n_{2}\right)} C_{\left[(1,0)^{\left.n_{1},(0,1)^{n_{2}}\right]}\right.}^{\mathbf{p}}=\sum_{\mathbf{p} \vdash\left(m_{1}, m_{2}\right)} C_{\mathbf{q}}^{\mathbf{p}}=\sum_{\pi \in \Pi(n)} C_{\pi_{n}}^{\pi}=\sum_{\pi^{\prime} \in \Pi(m)} C_{\pi}^{\pi^{\prime}}
$$

Finally, in (2.20) we gave the 1-matrix basis change from the monomial basis to the multitrace basis in terms of characters of $S_{n}$ representations and the Kostka numbers. Comparing with the (1-matrix equivalent of) definition (4.23), we see

$$
C_{q}^{p}=\frac{1}{\prod_{i} p_{i} !} \sum_{R \vdash n} \chi_{R}(q) K_{R p}
$$

\section{Counting: $\mathrm{U}(2) \times \mathrm{U}(N)$ Young diagram labels and multiplicities at weak coupling}

The space of states spanned by symmetrised traces $T_{\mathbf{p}}$ of general matrices $X, Y$ admits a $\mathrm{U}(2)$ action on the pair $X, Y$ as in section 2.2. These symmetrised traces are representatives of the elements of the ring of gauge invariants modulo commutators. Specialising to diagonal matrices $X=\operatorname{Diag}\left(x_{1}, x_{2}, \cdots, x_{N}\right), Y=\operatorname{Diag}\left(y_{1}, y_{2}, \cdots, y_{N}\right)$ gives the isomorphism [35, 37] to multi-symmetric polynomials in $x_{i}, y_{i}$ discussed in section 3.2. For economy of notation, we are generally using $T_{\mathbf{p}}$ also for the image $\iota\left(T_{\mathbf{p}}\right)$ of the isomorphism. There is an analogous $\mathrm{U}(2)$ action on multi-symmetric functions which transforms the pairs $x_{i}, y_{i}$. Applying the isomorphism and then doing a $\mathrm{U}(2)$ transformation is equivalent to doing a $\mathrm{U}(2)$ transformation on symmetrised traces and then applying the isomorphism. In other words the isomorphism between gauge invariants modulo commutators and multisymmetric polynomials is a $\mathrm{U}(2)$ equivariant isomorphism. The $\mathrm{U}(2)$ transformations $(2.54)$ on the monomial multi-symmetric functions, $M_{\mathbf{p}}$, are obtained either by expressing them in terms of $T_{\mathbf{p}}$ using the $\widetilde{C}$ transformation or equivalently using the $\mathrm{U}(2)$ on the pairs $\left(x_{i}, y_{i}\right)$. 
In this latter picture, the $\mathrm{U}(2)$ generators are

$$
\begin{aligned}
\mathcal{J}_{0} & =\sum_{i=1}^{N}\left(x_{i} \frac{\partial}{\partial x_{i}}+y_{i} \frac{\partial}{\partial y_{i}}\right) & \mathcal{J}_{3} & =\sum_{i=1}^{N}\left(x_{i} \frac{\partial}{\partial x_{i}}-y_{i} \frac{\partial}{\partial y_{i}}\right) \\
\mathcal{J}_{+} & =\sum_{i=1}^{N} x_{i} \frac{\partial}{\partial y_{i}} & \mathcal{J}_{-} & =\sum_{i=1}^{N} y_{i} \frac{\partial}{\partial x_{i}}
\end{aligned}
$$

A U(2) covariant basis will be sorted by $\mathrm{U}(2)$ representations $\Lambda$ and an index $M_{\Lambda}$ labelling the basis states. As in section $2.2, M_{\Lambda}$ runs over the semi-standard tableaux of shape $\Lambda$ and determines the field content. In order to parameterise the space for a specific $\Lambda$, we observe that for each vector partition $\mathbf{p} \vdash\left(n_{1}, n_{2}\right)$, there is an associated integer partition $p(\mathbf{p}) \vdash n_{1}+n_{2}$ obtained by summing the pairs

$$
\mathbf{p}=\left[\left(\lambda_{1}, \mu_{1}\right),\left(\lambda_{2}, \mu_{2}\right), \ldots,\left(\lambda_{k}, \mu_{k}\right)\right] \rightarrow p(\mathbf{p})=\left[\lambda_{1}+\mu_{1}, \lambda_{2}+\mu_{2}, \ldots, \lambda_{k}+\mu_{k}\right]
$$

Consider the action of $\mathrm{U}(2)$ on a simple monomial $x^{\lambda} y^{\mu}$. We have

$$
\begin{array}{ll}
\mathcal{J}_{0} x^{\lambda} y^{\mu}=(\lambda+\mu) x^{\lambda} y^{\mu} & \mathcal{J}_{3} x^{\lambda} y^{\mu}=(\lambda-\mu) x^{\lambda} y^{\mu} \\
\mathcal{J}_{+} x^{\lambda} y^{\mu}=\mu x^{\lambda+1} y^{\mu-1} & \mathcal{J}_{-} x^{\lambda} y^{\mu}=\lambda x^{\lambda-1} y^{\mu+1}
\end{array}
$$

The operators $\mathcal{J}_{ \pm}$send $\lambda \rightarrow \lambda \pm 1, \mu \rightarrow \mu \mp 1$ while $\mathcal{J}_{0}$ and $\mathcal{J}_{3}$ leave $\lambda, \mu$ invariant. For all $\mathrm{U}(2)$ generators, the sum $\lambda+\mu$ is unchanged. More generally, for a monomial $x_{1}^{\lambda_{1}} y_{1}^{\mu_{1}} \ldots x_{k}^{\lambda_{k}} y_{k}^{\mu_{k}}$, the sums $\lambda_{i}+\mu_{i}$ are unchanged in each monomial term arising from the action of the $\mathrm{U}(2)$ generators.

Applying this analysis to each of the monomials in $M_{\mathbf{p}}$, we see that $\mathrm{U}(2)$ preserves the associated partition $p(\mathbf{p})$, and therefore $p$ serves as another label in the $\mathrm{U}(2)$ covariant basis. We denote the multiplicity of a given pair $\Lambda, p$ in the covariant monomial basis by $\mathcal{M}_{\Lambda, p}$.

For a given associated partition $p=<p_{1}, p_{2}, \cdots>$ we have monomial multi-symmetric functions $M_{\mathbf{p}}$ with $p(\mathbf{p})=p$. The constituent monomials in $M_{\mathbf{p}}$ (recall the defining equation (2.10)) contain products of $p_{i}$ factors each with $i$ variables that can be $x$ or $y$ and are transformed between the two using $\mathcal{J}_{ \pm}$. We will show that these fit into the representation

$$
\mathcal{R}_{p}^{\mathrm{U}(2)}=\bigotimes_{i} \operatorname{Sym}^{p_{i}}\left(\operatorname{Sym}^{i}\left(V_{2}\right)\right)
$$

where $V_{2}$ is the 2-dimensional fundamental representation of $\mathrm{U}(2)$. We can decompose $\mathcal{R}_{p}^{\mathrm{U}(2)}$ in terms of irreducible representations $R_{\Lambda}^{\mathrm{U}(2)}$

$$
\mathcal{R}_{p}^{\mathrm{U}(2)}=\bigoplus_{\substack{\Lambda \vdash n \\ l(\Lambda) \leq 2}} R_{\Lambda}^{\mathrm{U}(2)} \otimes V_{\Lambda, p}^{\text {mult }}
$$

for some multiplicity space $V_{\Lambda, p}^{\text {mult }}$. The direct sum is restricted to run only over $\Lambda \vdash n$ since $\mathcal{R}_{p}^{\mathrm{U}(2)}$ is a subspace of $\left(V_{2}\right)^{\otimes n}$, and therefore the $\mathrm{U}(1)$ weight of all sub-representations is $n$. The analogous representation of the global symmetry $\mathrm{U}(3)$ in the case of eighth-BPS 
states is discussed in [32,33]. The multiplicity of $R_{\Lambda}^{\mathrm{U}(2)}$ in $\mathcal{R}_{p}^{\mathrm{U}(2)}$ is just the dimension of the multiplicity space $V_{\Lambda, p}^{\text {mult }}$, and is also the multiplicity of the pair $\Lambda, p$ in the covariant monomial basis

$$
\mathcal{M}_{\Lambda, p}=\operatorname{Mult}\left(\Lambda, \mathcal{R}_{p}^{\mathrm{U}(2)}\right)=\operatorname{Dim}\left(V_{\Lambda, p}^{m u l t}\right)
$$

To find this multiplicity we split $\mathrm{U}(2)$ into its $\mathrm{U}(1)$ and $\mathrm{SU}(2)$ components as discussed in section 2.2.2. As already mentioned, $\mathcal{R}_{p}^{\mathrm{U}(2)}$ is in the weight $n$ representation of $\mathrm{U}(1)$, so

$$
\mathcal{R}_{p}^{\mathrm{U}(2)}=R_{n}^{\mathrm{U}(1)} \otimes \mathcal{R}_{p}^{\mathrm{SU}(2)}
$$

where $\mathcal{R}_{p}^{\mathrm{SU}(2)}$ is

$$
\mathcal{R}_{p}^{\mathrm{SU}(2)}=\bigotimes_{i} \operatorname{Sym}^{p_{i}}\left(\operatorname{Sym}^{i}\left(R_{\frac{1}{2}}\right)\right)=\bigotimes_{i} \operatorname{Sym}^{p_{i}}\left(R_{\frac{i}{2}}\right)
$$

for $R_{j}$ the spin $j$ representation of $\mathrm{SU}(2)$. Then the $\mathrm{U}(2)$ decomposition (5.5) of $\mathcal{R}_{p}^{\mathrm{U}(2)}$ is equivalent to the $\mathrm{SU}(2)$ decomposition

$$
\mathcal{R}_{p}^{\mathrm{SU}(2)}=\bigoplus_{j} R_{j} \otimes V_{\left[\frac{n}{2}+j, \frac{n}{2}-j\right], p}^{m u l t}
$$

where we have used the correspondence, discussed in section 2.2.2, between a U(2) representation $\Lambda=\left[\frac{n}{2}+j, \frac{n}{2}-j\right]$ of $\mathrm{U}(1)$ weight $n$ and an $\mathrm{SU}(2)$ representation of spin $j$. The question of calculating the dimension of the multiplicity space in (5.9) is called an $\mathrm{SU}(2)$ plethysm problem and is addressed in [58]. We will use a formula derived there shortly.

The monomials $M_{\mathbf{p}}$ with $p(\mathbf{p})=p$ define states $|\mathbf{p}\rangle$ in $\mathcal{R}_{p}^{\mathrm{U}(2)}$, whose normalisation is given by the $S_{n}$ inner product on $M_{\mathbf{p}}$

$$
\langle\mathbf{p} \mid \mathbf{q}\rangle=\left\langle M_{\mathbf{p}} \mid M_{\mathbf{q}}\right\rangle
$$

There is a change of basis to $\mathrm{U}(2)$ orthonormal covariant states of the form

$$
\left|\Lambda, M_{\Lambda}, p, \nu\right\rangle
$$

where $\nu$ is a multiplicity index with $1 \leq \nu \leq \mathcal{M}_{\Lambda, p}$. This change of basis is implemented using Clebsch-Gordan coefficients

$$
\left|\Lambda, M_{\Lambda}, p, \nu\right\rangle=\sum_{\mathbf{p}: p(\mathbf{p})=p} B_{\Lambda, M_{\Lambda}, p, \nu}^{\mathbf{p}}|\mathbf{p}\rangle
$$

We define the covariant monomial operators by

$$
M_{\Lambda, M_{\Lambda}, p, \nu}=\sum_{\mathbf{p}: p(\mathbf{p})=p} B_{\Lambda, M_{\Lambda}, p, \nu}^{\mathbf{p}} M_{\mathbf{p}}
$$

As an example, consider the multi-symmetric monomials for field content $(2,2)$, given explicitly in (4.91)-(4.99). We only give the $M_{\Lambda}$ and $p$ labels, as the shape of the Young 
tableau specifies $\Lambda$, and the multiplicity for these operators is trivial. The covariant monomials are

$$
\begin{aligned}
& M_{[1|1| 2 \mid 2,[4]}=\sqrt{\frac{3}{2}} M_{[(2,2)]} \\
& M_{[1|1| 2 \mid 2,[3,1]}=\sqrt{\frac{3}{14}}\left(M_{[(2,1),(0,1)]}+M_{[(1,2),(1,0)]}\right) \\
& M_{[1[12[2],[2,2]}=\frac{1}{3 \sqrt{2}}\left(M_{[(2,0),(0,2)]}+2 M_{[(1,1),(1,1)]}\right) \\
& \left.M_{\left[\frac{1|1| 2 \mid 2,[2,1,1]}{4 \sqrt{15}}\right.}=\frac{1}{4[(2,0),(0,1),(0,1)]}+4 M_{[(1,1),(1,0),(0,1)]}+M_{[(1,0),(1,0),(0,2)]}\right) \\
& M_{[1|1| 2 \mid 2,[1,1,1,1]}=\frac{1}{4 \sqrt{6}} M_{[(1,0),(1,0),(0,1),(0,1)]} \\
& M_{\left[\frac{1}{2}[1 \mid 2,[3,1]\right.}=\frac{1}{\sqrt{2}}\left(M_{[(2,1),(0,1)]}-M_{[(1,2),(1,0)]}\right) \\
& M_{\frac{1 \frac{1}{2}[12],[2,1,1]}{4}}=\frac{1}{4}\left(M_{[(2,0),(0,1),(0,1)]}-M_{[(1,0),(1,0),(0,2)]}\right) \\
& M_{\frac{\frac{11}{2 \frac{1}{2}},[2,2]}{\sqrt{6}}}=\frac{1}{\sqrt{[(2,0),(0,2)]}}\left(M_{[(1,1),(1,1)]}\right) \\
& M_{\frac{\frac{1}{2} \frac{1}{2},[2,1,1]}{6}}=\frac{1}{6}\left(M_{[(2,0),(0,1),(0,1)]}-2 M_{[(1,1),(1,0),(0,1)]}+M_{[(1,0),(1,0),(0,2)]}\right)
\end{aligned}
$$

The associated partition has length $l(p(\mathbf{p}))=l(\mathbf{p})$, and therefore the SEP compatibility (modulo commutators) of the $M_{\mathbf{p}}$ basis is transferred to the new basis.

If $p$ has length $l(p)>N$ then the multi-symmetric function $M_{\Lambda, M_{\Lambda}, p, \nu}$ vanishes identically, while on the other side of the isomorphism, the operator $M_{\Lambda, M_{\Lambda}, p, \nu}$ reduces to a commutator trace and therefore is no longer pre-BPS. Operators with $l(p) \leq N$ are in general not pre-BPS, but differ from such an operator by a commutator trace. In section 6 we show how to remove this commutator trace component to derive a pre-BPS basis. For now, we note that the multiplicity $\mathcal{M}_{\Lambda, p}$ determines the finite $N$ combinatorics of the quarter-BPS sector.

The half-BPS operators $\mathcal{O}_{R}$ defined in (2.18) are dual to giant gravitons. There are two types of giant gravitons: those that have an extended $S^{3} \subset S^{5}$ as part of the world-volume, and those that have an extended $S^{3} \subset A d S_{5}$. We will refer to these as sphere giants and AdS giants respectively: they are also sometimes distinguished in the AdS/CFT literature as giants versus dual-giants respectively. $\mathrm{U}(2)$ rotations of these half-BPS giants produces giant graviton states in the $\Lambda=[n]$ representation, where $n$ is the number of boxes in the Young diagram $R$. The nature of the Young diagram $R$ is related to the type of giant graviton system. As we deform $\Lambda$ to $\Lambda=[n-m, m]$ we move away from the half-BPS sector. The deformations of sphere giant states are described in terms of moduli spaces of polynomials in three complex variables [15] while deformations of AdS giant states are described in terms of a family of solutions with $S^{3} \subset A d S_{5}$ world-volumes orbiting great circles on the $S^{5}[18]$.

In section 6 we will produce a basis $S_{\Lambda, M_{\Lambda}, p, \nu}^{\mathrm{BPS}}$ for the quarter-BPS sector with the same labels as (5.13). For $\Lambda=[n]$, this basis agrees with the half-BPS Schur basis (2.18) by 
identifying $p$ with $R$. This matching between the Young diagrams $p$ labelling the quarterBPS sector and $R$ labelling the half-BPS states suggests that for a particular diagram $p$, we can follow the half-BPS sector states into the quarter-BPS sector by considering $\Lambda=[n-m, m]$ and slowly increasing the length of the second row, $m$. We expect that if we keep $p$ fixed in this half to quarter transition, we qualitatively preserve the physical nature of the giant graviton: Young diagrams with a few long rows of lengths order $N$ correspond to AdS-giants while diagrams with a few long columns of lengths order $N$ correspond to sphere giants. It is reasonable to think of Young diagrams $p$ (for more general $\Lambda$ ) with $k$ rows of length comparable to $N$ as an AdS-giant system formed as some form of composite of $k$ giants. Likewise, in the following discussion, we will think of a Young diagram $p$ with $k$ rows of length order $N$ as some composite involving $k$ sphere giants. There will be interesting differences between sphere giants and AdS giants, so the precise meaning of "composite system of $k$ giants" is something which should be explored through future comparisons between bulk physics and CFT correlators.

The multiplicities (5.6) interpolate from half-BPS in the case $\Lambda=[n]$ to more general quarter-BPS for $\Lambda=\left[n-\Lambda_{2}, \Lambda_{2}\right]$, with small $\Lambda_{2}$ being close to half-BPS. These multiplicities should be reproducible from the stringy physics of $D 3$-branes in $A d S_{5} \times S^{5}$. In each part of this section, we discuss the giant graviton interpretation of the multiplicity results.

\section{$5.1 \Lambda, p$ multiplicities and plethysms of SU(2) characters}

We consider the space of multi-symmetric functions $M_{\mathbf{p}}$ with a given associated partition $p$, and how this can be split into $\mathrm{U}(2)$ representations.

As discussed in section 2.2, $\mathrm{U}(2)$ can be split into a product of $\mathrm{U}(1)$ and $\mathrm{SU}(2)$. The $\mathrm{U}(1)$ weight of a given $p$ is just $n=|p|$, so to derive the $\mathrm{U}(2)$ representation we first study the $\mathrm{SU}(2)$ part, then recombine with the $\mathrm{U}(1)$ piece at the end.

For the sake of simplicity, we will primarily work with non-symmetrised monomials, since such a choice determines the associated multi-symmetric function by adding all permuted monomials. The construction of the multi-symmetric function from the nonsymmetrised monomial can affect the $\mathrm{SU}(2)$ structure, and we will describe this in more detail as it occurs.

Start by considering $p=[n]$. This allows $\mathbf{p}=[(\lambda, \mu)]$ for $\lambda+\mu=n$. The nonsymmetrised monomials corresponding to these are just $x_{1}^{\lambda} y_{1}^{\mu}$, whose action under $\mathrm{U}(2)$ we gave in (5.3). From the action of $\mathcal{J}_{ \pm}, \mathcal{J}_{3}$, they lie in the spin $\frac{n}{2}$ representation of $\mathrm{SU}(2)$. Symmetrising the monomials does not change the $\mathrm{SU}(2)$ structure, so $p=[n]$ produces the $R_{\frac{n}{2}}$ representation of $\mathrm{SU}(2)$.

Next consider $p=\left[k_{1}, k_{2}\right]$. This allows $\mathbf{p}=\left[\left(\lambda_{1}, \mu_{1}\right),\left(\lambda_{2}, \mu_{2}\right)\right]$, with corresponding non-symmetrised monomials $x_{1}^{\lambda_{1}} y_{1}^{\mu_{1}} x_{2}^{\lambda_{2}} y_{2}^{\mu_{2}}$ subject to $\lambda_{i}+\mu_{i}=k_{i}$ for $i=1,2$. There are $(k+1)(l+1)$ different states, living in the tensor product representation $R_{\frac{k_{1}}{2}} \otimes R_{\frac{k_{2}}{2}}$. When $k_{1} \neq k_{2}$, this is the correct $\mathrm{SU}(2)$ representation for the symmetrised version as well. However, if $k_{1}=k_{2}$, then the states $x_{1}^{\lambda_{1}} y_{1}^{\mu_{1}} x_{2}^{\lambda_{2}} y_{2}^{\mu_{2}}$ and $x_{1}^{\lambda_{2}} y_{1}^{\mu_{2}} x_{2}^{\lambda_{1}} y_{2}^{\mu_{1}}$ both lead to the same multi-symmetric function and should be identified with each other. The correct representation here is the symmetric part of the tensor product, written as $\operatorname{Sym}^{2}\left(R_{\frac{k_{1}}{2}}\right)$. 
As our final example, take $p=\left[k_{1}, k_{2}, k_{3}\right]$, allowing $\mathbf{p}=\left[\left(\lambda_{1}, \mu_{1}\right),\left(\lambda_{2}, \mu_{2}\right),\left(\lambda_{3}, \mu_{3}\right)\right]$. By the same considerations as the previous two examples, the non-symmetrised monomials $x_{1}^{\lambda_{1}} y_{1}^{\mu_{1}} x_{2}^{\lambda_{2}} y_{2}^{\mu_{2}} x_{3}^{\lambda_{3}} y_{3}^{\lambda_{3}}$ fit into the $R_{\frac{k_{1}}{2}} \otimes R_{\frac{k_{2}}{2}} \otimes R_{\frac{k_{3}}{2}}$ representation of $\mathrm{SU}(2)$. If all three of the $k \mathrm{~s}$ are distinct, this is the correct representation for the symmetrised monomials. If two of the $k_{\mathrm{s}}$ coincide and the third is distinct, e.g. $k_{1}=k_{2} \neq k_{3}$, then the $M_{\mathbf{p}}$ live in $\operatorname{Sym}^{2}\left(R_{\frac{k_{1}}{2}}\right) \otimes R_{\frac{k_{3}}{2}}$. Finally, if $k_{1}=k_{2}=k_{3}$, then there are 6 permutations of the basic monomial that lead to the same multi-symmetric function and should be identified. These are

$$
\begin{array}{lll}
x_{1}^{\lambda_{1}} y_{1}^{\mu_{1}} x_{2}^{\lambda_{2}} y_{2}^{\mu_{2}} x_{3}^{\lambda_{3}} y_{3}^{\lambda_{3}} & x_{1}^{\lambda_{2}} y_{1}^{\mu_{2}} x_{2}^{\lambda_{3}} y_{2}^{\mu_{3}} x_{3}^{\lambda_{1}} y_{3}^{\lambda_{1}} & x_{1}^{\lambda_{3}} y_{1}^{\mu_{3}} x_{2}^{\lambda_{1}} y_{2}^{\mu_{1}} x_{3}^{\lambda_{2}} y_{3}^{\lambda_{2}} \\
x_{1}^{\lambda_{1}} y_{1}^{\mu_{1}} x_{2}^{\lambda_{3}} y_{2}^{\mu_{3}} x_{3}^{\lambda_{2}} y_{3}^{\lambda_{2}} & x_{1}^{\lambda_{3}} y_{1}^{\mu_{3}} x_{2}^{\lambda_{2}} y_{2}^{\mu_{2}} x_{3}^{\lambda_{1}} y_{3}^{\lambda_{1}} & x_{1}^{\lambda_{2}} y_{1}^{\mu_{2}} x_{2}^{\lambda_{1}} y_{2}^{\mu_{1}} x_{3}^{\lambda_{3}} y_{3}^{\lambda_{3}}
\end{array}
$$

In an analogous way to the single coincidence, this leads to us using the completely symmetric part of the triple tensor product, written $\operatorname{Sym}^{3}\left(R_{\frac{k_{1}}{2}}\right)$. This is the part of $R_{\frac{k_{1}}{2}}^{\otimes 3}$ that is invariant under all $S_{3}$ permutations.

From the principles established in these three examples, we can generalise to a generic integer partition $p$. The multi-symmetric functions with associated partition $p=<p_{1}, p_{2}, \cdots>$ fit into the representation of $\mathrm{SU}(2)$ given by

$$
\mathcal{R}_{p}^{\mathrm{SU}(2)}=R_{p}^{\mathrm{SU}(2)}=\bigotimes_{i} \operatorname{Sym}^{p_{i}}\left(R_{\frac{i}{2}}\right)
$$

Restoring the $\mathrm{U}(1)$ weight, as a $\mathrm{U}(2)$ representation this is

$$
\begin{aligned}
\mathcal{R}_{p}^{\mathrm{U}(2)} & =R_{n}^{\mathrm{U}(1)} \otimes \mathcal{R}_{p}^{\mathrm{SU}(2)} \\
& =\bigotimes_{i} R_{i p_{i}}^{\mathrm{U}(1)} \otimes \operatorname{Sym}^{p_{i}}\left(\operatorname{Sym}^{i}\left(R_{\frac{1}{2}}\right)\right) \\
& =\bigotimes_{i} \operatorname{Sym}^{p_{i}}\left(R_{i}^{\mathrm{U}(1)} \otimes \operatorname{Sym}^{i}\left(R_{\frac{1}{2}}\right)\right) \\
& =\bigotimes_{i} \operatorname{Sym}^{p_{i}}\left(\operatorname{Sym}^{i}\left(R_{1}^{\mathrm{U}(1)} \otimes R_{\frac{1}{2}}\right)\right) \\
& =\bigotimes_{i} \operatorname{Sym}^{p_{i}}\left(\operatorname{Sym}^{i}\left(V_{2}\right)\right)
\end{aligned}
$$

where we have used $R_{\frac{i}{2}}=\operatorname{Sym}^{i}\left(R_{\frac{1}{2}}\right)$ for $\mathrm{SU}(2)$ representations and the fundamental representation of $\mathrm{U}(2)$ is $V_{2}=R_{1}^{\mathrm{U}(1)} \otimes R_{\frac{1}{2}}$.

So the problem of finding $\mathcal{M}_{\Lambda, p}$ reduces to a $\mathrm{U}(2)$ representation theory problem of finding the multiplicity of $R_{\Lambda}^{\mathrm{U}(2)}$ within the representation $\mathcal{R}_{p}^{\mathrm{U}(2)}$, or equivalently the $\mathrm{SU}(2)$ representation theory problem of finding the multiplicity of $R_{j}$ within $\mathcal{R}_{p}^{\mathrm{SU}(2)}$ and using the correspondence $j \leftrightarrow \Lambda=\left[\frac{n}{2}+j, \frac{n}{2}-j\right]$ for $\mathrm{U}(2)$ representations of $\mathrm{U}(1)$ weight $n$.

We will solve the $\mathrm{SU}(2)$ problem. In order to do this, we calculate the character of the representation (5.24) and compare it to the known characters of the spin representations. From standard SU(2) representation theory we know that

$$
\chi_{R_{j}}\left(q^{J_{3}}\right)=q^{j}+q^{j-1}+\cdots+q^{-j}=q^{-j} \frac{\left(1-q^{2 j+1}\right)}{(1-q)}
$$


So the multiplicity of $R_{j}$ inside a direct sum representation $R$ is given by

$$
\operatorname{Mult}\left(R_{j}, R\right)=\operatorname{Coeff}\left[q^{-j},(1-q) \chi_{R}\left(q^{J_{3}}\right)\right]
$$

Taking a single factor of $(5.24)$, the character of $\mathrm{Sym}^{p_{i}}\left(R_{\frac{i}{2}}\right)$ was calculated in [58] and is given by

$$
\begin{aligned}
\chi_{\operatorname{Sym}^{p_{i}}\left(R_{\frac{i}{2}}\right)}\left(q^{J_{3}}\right) & =q^{-\frac{i p_{i}}{2}} \frac{\left(1-q^{p_{i}+1}\right)}{(1-q)} \frac{\left(1-q^{p_{i}+2}\right)}{\left(1-q^{2}\right)} \cdots \frac{\left(1-q^{p_{i}+i}\right)}{\left(1-q^{i}\right)} \\
& =q^{-\frac{i p_{i}}{2}} F_{i, p_{i}}(q)
\end{aligned}
$$

where

$$
F_{i, p_{i}}=\frac{\left(1-q^{p_{i}+1}\right)}{(1-q)} \frac{\left(1-q^{p_{i}+2}\right)}{\left(1-q^{2}\right)} \cdots \frac{\left(1-q^{p_{i}+i}\right)}{\left(1-q^{i}\right)}
$$

So the multiplicity of $R_{j}$ inside $\mathcal{R}_{p}^{\mathrm{SU}(2)}$ is

$$
\begin{aligned}
\operatorname{Mult}\left(R_{j}, \mathcal{R}_{p}^{\mathrm{SU}(2)}\right) & =\operatorname{Coeff}\left(q^{-j},(1-q) \prod_{i} q^{-\frac{i p_{i}}{2}} F_{i, p_{i}}(q)\right) \\
& =\operatorname{Coeff}\left(q^{-j},(1-q) q^{-\frac{n}{2}} \prod_{i} F_{i, p_{i}}(q)\right)
\end{aligned}
$$

Since the $\Lambda=[n-m, m]$ representation of $\mathrm{U}(2)$ corresponds to spin $j=\frac{n}{2}-m$, this means

$$
\begin{aligned}
\operatorname{Mult}\left([n-m, m], \mathcal{R}_{p}^{\mathrm{U}(2)}\right) & =\operatorname{Coeff}\left(q^{m-\frac{n}{2}},(1-q) q^{-\frac{n}{2}} \prod_{i} F_{i, p_{i}}(q)\right) \\
& =\operatorname{Coeff}\left(q^{m},(1-q) \prod_{i} F_{i, p_{i}}(q)\right)
\end{aligned}
$$

Writing

$$
F_{p}(q)=\prod_{i} F_{i, p_{i}}(q)
$$

we can give a simple formula for the multiplicity in terms of the coefficients of $F_{p}$

$$
\mathcal{M}_{[n-m, m], p}=\operatorname{Coeff}\left(q^{m}, F_{p}\right)-\operatorname{Coeff}\left(q^{m-1}, F_{p}\right)
$$

We now take two distinct approaches to studying $F_{p}$. Firstly we derive a generic formula for $\mathcal{M}_{\Lambda, p}$ that allows simple computational calculations of the multiplicity for any $\Lambda, p$ of reasonable size. Secondly, we study sets of $p$ which have identical multiplicities for all $\Lambda$ and give explicit results of $\mathcal{M}_{\Lambda, p}$ for the simplest such sets.

\subsection{Covariant trace bases}

In the previous section we argued from the vector partition structure of the monomial multisymmetric functions that the $M_{\mathbf{p}}$ fit in to the representation $\mathcal{R}_{p}^{\mathrm{U}(2)}$ of $\mathrm{U}(2)$, where $p$ is the 
integer partition associated to p. Performing a similar process on the multi-trace multisymmetric functions $T_{\mathbf{p}}$ (or equivalently symmetrised trace operators), the $\mathrm{U}(2)$ action not only preserves $p(\mathbf{p})$, it has exactly the same form as the action on monomials $M_{\mathbf{p}}$. That is, given $\mathcal{U} \in \mathrm{U}(2)$ with action

$$
\mathcal{U} M_{\mathbf{p}}=\sum_{\mathbf{q}} a_{\mathbf{p}}^{\mathbf{q}} M_{\mathbf{q}}
$$

for some coefficients $a_{\mathbf{p}}^{\mathbf{q}}$, then the action of $\mathcal{U}$ on symmetrised traces is

$$
\mathcal{U} T_{\mathbf{p}}=\sum_{\mathbf{q}} a_{\mathbf{p}}^{\mathbf{q}} T_{\mathbf{q}}
$$

Therefore sorting $M_{\mathbf{p}}$ into a $\mathrm{U}(2)$ covariant basis is mathematically identical to sorting $T_{\mathbf{p}}$ into a $\mathrm{U}(2)$ covariant basis. It follows that the linear maps $C, \widetilde{C}$ relating $M_{\mathbf{p}}$ and $T_{\mathbf{p}}$ are $\mathrm{U}(2)$ equivariant, and we can define a $\mathrm{U}(2)$ covariant symmetrised trace basis

$$
T_{\Lambda, M_{\Lambda}, p, \nu}=\sum_{\mathbf{p}: p(\mathbf{p})=p} B_{\Lambda, M_{\Lambda}, p, \nu}^{\mathbf{p}} T_{\mathbf{p}}
$$

In [32], the authors proved that the multiplicity of $\Lambda, p$ in the symmetrised trace covariant basis is

$$
\mathcal{M}_{\Lambda, p}=\chi_{\Lambda}\left(\mathbb{P}_{p}\right)
$$

where $\chi_{\Lambda}$ is the $S_{n}$ character of $\Lambda$ and $\mathbb{P}_{p}$ is an element of $\mathbb{C}\left(S_{n}\right)$ that projects onto symmetrised traces with cycle type $p$. We discuss this projector in section 5.7.

The formulae (5.33) and (5.37) give $\mathcal{M}_{\Lambda, p}$ from $\mathrm{U}(2)$ and $S_{n}$ representation theory respectively. The former is more amenable to explicit calculations.

As discussed above (4.23), the symmetrised trace operators $T_{\mathbf{p}}$ with $l(\mathbf{p}) \leq N$ form a basis for symmetrised traces (but not pre-BPS operators) at finite $N$. Since the $p$ label in (5.36) has the same length as $\mathbf{p}$, this property also holds for $T_{\Lambda, M_{\Lambda}, p, \nu}$.

In addition to the symmetrised trace covariant basis, there is a corresponding $\mathrm{U}(2)$ covariant basis for commutator traces. It follows from the definitions (2.54) that the $\mathrm{U}(2)$ generators act on a simple commutator as

$$
R_{j}^{i}[X, Y]=\delta_{j}^{i}[X, Y]
$$

Any commutator trace, generically containing a more complicated commutator than $[X, Y]$, can be written as a linear combination of traces containing $[X, Y]$. So (5.38) shows that the space of commutator traces forms a $\mathrm{U}(2)$ representation. By similar considerations to $M_{\mathbf{p}}$ and $T_{\mathbf{p}}$, these can be further sorted by an integer partition $p \vdash n$ that describes the factorisation of a commutator multi-trace into single traces.

In [22], the authors used superspace techniques in the $\mathrm{SU}(N)$ gauge theory to develop candidate quarter-BPS operators and SUSY descendent operators. These are exactly the covariant symmetrised trace and commutator trace bases respectively, though they did not include partitions with components of size 1 , since in the $\mathrm{SU}(N)$ theory, traces of individual matrices vanish.

The covariant bases for symmetrised and commutator traces are used in appendices A, $\mathrm{B}$ and $\mathrm{C}$ to describe the final BPS operators at $n=5,6$. In this section we will focus 
on the covariant monomials and not comment further on the covariant symmetrised or commutator traces.

\subsection{General multiplicity formula}

We now find an expression for

$$
\text { Coeff }\left(q^{m}, F_{p}(q)\right)
$$

This is done explicitly for $m=0,1,2,3$, from which we extrapolate the general result.

The relevant parts of $p$ to describe the coefficients in (5.32) are

$$
c_{j, k}=\left|\left\{i: i>j, p_{i} \geq k\right\}\right| \quad j \geq 0, k \geq 1
$$

Let $Y_{j}(p), j \geq 0$ be the Young diagram of $p$ with the first $j$ columns removed. Then intuitively, $c_{j, k}$ is the number of vertical edges of length $k$ or greater in $Y_{j}(p)$. It follows that $c_{j, 1}$ is the number of corners in $Y_{j}(p)$, and $c_{0,1}$ is the number of corners in the full Young diagram $Y(p)$. Figure 3 shows some examples to illustrate this. The full set of $c_{j, k}$ completely determines the partition $p$.

It will also be useful to define

$$
s_{l}=\sum_{j+k=l} c_{j, k}
$$

We have included examples of the $s_{l}$ in figure 3 . In contrast to the $c_{j, k}$, the $s_{l}$ do not define the partition $p$. For example, $p=[2]$ and $p=[1,1]$ both have $s_{1}=1, s_{2}=1$ and all others zero. The sets of partitions which have identical $s_{l}$ for all $l$ are studied in section 5.4.

To find the coefficients of $q^{0,1,2,3}$ in $F_{p}(q)$, we look at the low order terms from the definitions (5.29) and (5.32). For all $i>0, F_{i, p_{i}}$ contains the factor

$$
\frac{\left(1-q^{p_{i}+1}\right)}{(1-q)}=1+q+q^{2}+\cdots+q^{p_{i}}
$$

For all $i>1, F_{i, p_{i}}$ contains, in addition to the above, the factor

$$
\begin{aligned}
\frac{\left(1-q^{p_{i}+2}\right)}{\left(1-q^{2}\right)} & =\left(1+q^{2}+q^{4}+\cdots\right)\left(1-q^{p_{i}+2}\right) \\
& =1+q^{2}-q^{p_{i}+2}+O\left(q^{4}\right)
\end{aligned}
$$

For all $i>2$, the factor $F_{i, p_{i}}$ contains, in addition to the above,

$$
\begin{aligned}
\frac{\left(1-q^{p_{i}+3}\right)}{\left(1-q^{3}\right)} & =\left(1+q^{3}+\cdots\right)\left(1-q^{p_{i}+3}\right) \\
& =1+q^{3}-q^{p_{i}+3}+O\left(q^{4}\right)
\end{aligned}
$$

All other factors in the definition (5.29) of $F_{i, p_{i}}$ are of the form $1+O\left(q^{4}\right)$ so we can ignore them for our purposes, giving

$$
F_{p}=f_{1} f_{2} f_{3}+O\left(q^{4}\right)
$$




\begin{tabular}{|c|c|c|c|c|c|}
\hline$p$ & Young diagram & \multicolumn{3}{|c|}{ Table of $c_{j, k}$} & Table of $s_{l}$ \\
\hline$[4,3,2,1]$ & & $\begin{array}{l}c_{j, k} \\
j= \\
j= \\
j= \\
j=\end{array}$ & \begin{tabular}{l|l}
$c$ & $k=$ \\
0 & 4 \\
1 & 3 \\
2 & 2 \\
3 & 1
\end{tabular} & & $\begin{array}{l}s_{1}=4 \\
s_{2}=3 \\
s_{3}=2 \\
s_{4}=1\end{array}$ \\
\hline$[3,3,2,1,1]$ & & \begin{tabular}{c|c}
$c_{j, k}$ & $k$ \\
$j=0$ & \\
$j=1$ \\
$j=2$
\end{tabular} & $\begin{array}{l}c=1 k \\
3 \\
2 \\
1\end{array}$ & & $\begin{array}{l}s_{1}=3 \\
s_{2}=4 \\
s_{3}=2 \\
s_{4}=1\end{array}$ \\
\hline$[3,3,3]$ & & \begin{tabular}{c|r}
$c_{j, k}$ & $k=$ \\
$j=0$ & 1 \\
$j=1$ & 1 \\
$j=2$ & 1
\end{tabular} & $\begin{array}{r}1 k= \\
1 \\
1 \\
1\end{array}$ & $\begin{array}{c}k=3 \\
1 \\
1 \\
1\end{array}$ & $\begin{array}{l}s_{1}=1 \\
s_{2}=2 \\
s_{3}=3 \\
s_{4}=2 \\
s_{5}=1\end{array}$ \\
\hline
\end{tabular}

Figure 3. Examples of the non-zero $c_{j, k}$ and $s_{l}$ for various integer partitions $p$.

where

$$
\begin{aligned}
& f_{1}=\prod_{i>0}\left(1+q+q^{2}+\cdots+q^{p_{i}}\right) \\
& f_{2}=\prod_{i>1}\left(1+q^{2}-q^{p_{i}+2}+\cdots\right) \\
& f_{3}=\prod_{i>2}\left(1+q^{3}-q^{p_{i}+3}+\cdots\right)
\end{aligned}
$$

From this we can read off

$$
\operatorname{Coeff}\left(q^{0}, F_{p}\right)=1
$$

All the $q \mathrm{~s}$ in the expansion of $F_{p}$ come from $f_{1}$, with the coefficient given by the number of $p_{i} \geq 1$. From the definitions (5.40) and (5.41), we can express this as

$$
\operatorname{Coeff}\left(q, F_{p}\right)=c_{0,1}=s_{1}
$$

There are three ways to arrive at a $q^{2}$ from the product (5.47).

1. We can take a $q^{2}$ from a factor of $f_{2}$ and 1 from every other factor. Within $f_{2}$, this happens whenever $p_{i} \geq 1$ for $i>1$, so there are $c_{1,1}$ different ways of doing this. Therefore this route contributes $c_{1,1}$ to the coefficient of $q^{2}$.

2. We can take a $q^{2}$ from a factor of $f_{1}$. Each factor contains a $q^{2}$ term only if $p_{i} \geq 2$, so the number of different ways of doing this is $c_{0,2}$. 
3. We can take a $q$ from a pair of the $f_{1}$ factors. There are $\left(\begin{array}{c}c_{0,1} \\ 2\end{array}\right)$ different ways of doing this.

So we arrive at the expression

$$
\operatorname{Coeff}\left(q^{2}, F_{p}\right)=c_{0,2}+c_{1,1}+\left(\begin{array}{c}
c_{0,1} \\
2
\end{array}\right)=s_{2}+\frac{s_{1}\left(s_{1}-1\right)}{2}
$$

Looking at $q^{3}$, there are six distinct ways to arrive at a $q^{3}$ from the product (5.47).

1. We can take a $q^{3}$ from a factor of $f_{3}$. There are $c_{2,1}$ different ways of doing this.

2. We can take a $q^{3}$ from a factor of $f_{2}$. This can only be done if $p_{i}=1$, as it comes from the term $q^{p_{i}+1}$. The number of factors with $p_{i}=1$ is given by $c_{1,1}-c_{1,2}$. Noting that any $q^{3}$ obtained in this manner comes with a minus sign, this contributes $c_{1,2}-c_{1,1}$ to the coefficient.

3. We can take a $q^{3}$ from a factor of $f_{1}$. There are $c_{0,3}$ ways of doing this.

4. We can take a $q^{2}$ from a factor of $f_{2}$ and a $q$ from a factor of $f_{1}$. There are $c_{1,1} c_{0,1}$ ways of doing this.

5. We can take a $q^{2}$ from a factor of $f_{1}$ and a $q$ from a different factor of $f_{1}$. There are $c_{0,2}\left(c_{0,1}-1\right)$ different ways of doing this.

6. We can take a $q$ from three different factors of $f_{1}$. There are $\left(\begin{array}{c}c_{0,1} \\ 3\end{array}\right)$ different ways of doing this.

Collecting everything, we have

$$
\begin{aligned}
\operatorname{Coeff}\left(q^{3}, F_{p}\right) & =c_{2,1}+c_{1,2}-c_{1,1}+c_{0,3}+c_{1,1} c_{0,1}+c_{0,2}\left(c_{0,1}-1\right)+\left(\begin{array}{c}
c_{0,1} \\
3
\end{array}\right) \\
& =c_{2,1}+c_{1,2}+c_{0,3}+\left(c_{1,1}+c_{0,2}\right)\left(c_{0,1}-1\right)+\left(\begin{array}{c}
c_{0,1} \\
3
\end{array}\right) \\
& =s_{3}+s_{2}\left(s_{1}-1\right)+\frac{s_{1}\left(s_{1}-1\right)\left(s_{1}-2\right)}{6}
\end{aligned}
$$

A similar process for the coefficient of $q^{4}$ leads to

$$
\begin{aligned}
\operatorname{Coeff}\left(q^{4}, F_{p}\right)= & c_{0,4}+c_{1,3}+c_{2,2}+c_{3,1}+\left(c_{0,3}+c_{1,2}+c_{2,1}\right)\left(c_{0,1}-1\right) \\
& +\left(\begin{array}{c}
c_{0,2}+c_{1,1} \\
2
\end{array}\right)+\left(c_{0,2}+c_{1,1}\right)\left(\begin{array}{c}
c_{0,1}-1 \\
2
\end{array}\right)+\left(\begin{array}{c}
c_{0,1} \\
4
\end{array}\right) \\
= & s_{4}+s_{3}\left(s_{1}-1\right)+\frac{s_{2}\left(s_{2}-1\right)}{2}+\frac{s_{2}\left(s_{1}-1\right)\left(s_{1}-2\right)}{2} \\
& +\frac{s_{1}\left(s_{1}-1\right)\left(s_{1}-2\right)\left(s_{1}-3\right)}{24}
\end{aligned}
$$

In (5.51), (5.52), (5.53), (5.54) and (5.55) we have expressed the first 5 coefficients in the expansion of $F_{p}$ in terms of the $s_{l}$. The terms in these sums correspond to the partitions of 
the exponent of $q$. For example in (5.55), the terms correspond respectively to the partitions $[4],[3,1],[2,2],[2,1,1]$ and $[1,1,1,1]$. This leads us to suggest the general formula

$$
\operatorname{Coeff}\left(q^{m}, F_{p}\right)=\sum_{\lambda \vdash m}\left[\prod_{k}\left(s_{\lambda_{k}}-k+1\right)\right]\left[\prod_{i} \frac{1}{\mu_{i} !}\right]
$$

where we have used both the component notation $\lambda=\left[\lambda_{1}, \lambda_{2}, \ldots\right]$ and the multiplicity notation $\lambda=<\mu_{1}, \mu_{2}, \cdots>$ for $\lambda$.

In our work for this paper, we have algebraically proved this formula for $m \leq 6$, and have numerically checked it up to $m=20$. A proof for general $m$ and $p$ is a problem for future work.

It is interesting to note that since $F_{p}$ is a palindromic polynomial (arising from the $q \rightarrow q^{-1}$ invariance of $\mathrm{SU}(2)$ characters), these coefficients form a palindromic sequence. Explicitly,

$$
\operatorname{Coeff}\left(q^{m}, F_{p}\right)=\operatorname{Coeff}\left(q^{n-m}, F_{p}\right)
$$

As the sums over $\lambda$ in (5.56) get extremely complicated for large $m$, this is quite surprising, and leads us to suspect there is more hidden structure in the sum (5.56).

Combining (5.33) with (5.56) gives us an explicit formula for $\mathcal{M}_{\Lambda, p}$

$$
\begin{aligned}
\mathcal{M}_{[n-m, m], p}=\sum_{\lambda \vdash m}\left[\prod_{k}\left(s_{\lambda_{k}}-k+1\right)\right]\left[\prod_{i} \frac{1}{\mu_{i}(\lambda) !}\right] \\
\quad-\sum_{\lambda \vdash m-1}\left[\prod_{k}\left(s_{\lambda_{k}}-k+1\right)\right]\left[\prod_{i} \frac{1}{\mu_{i}(\lambda) !}\right]
\end{aligned}
$$

Applying this to $m=0$ to 4 , the formulae are

$$
\begin{aligned}
\mathcal{M}_{[n], p} & =1 \\
\mathcal{M}_{[n-1,1], p} & =s_{1}-1 \\
\mathcal{M}_{[n-2,2], p} & =s_{2}+\frac{s_{1}\left(s_{1}-3\right)}{2} \\
\mathcal{M}_{[n-3,3], p} & =s_{3}+s_{2}\left(s_{1}-2\right)+\frac{s_{1}\left(s_{1}-1\right)\left(s_{1}-5\right)}{6} \\
\mathcal{M}_{[n-4,4], p}= & s_{4}+s_{3}\left(s_{1}-2\right)+s_{2}\left(s_{2}-1\right)+\frac{s_{2}\left(s_{2}-1\right)}{2} \\
& +\frac{s_{2}\left(s_{1}-1\right)\left(s_{1}-4\right)}{2}+\frac{s_{1}\left(s_{1}-1\right)\left(s_{1}-2\right)\left(s_{1}-7\right)}{24}
\end{aligned}
$$

These formulae are independent of $N$, so to get finite $N$ multiplicities we impose the finite $N$ cut-off on $p$. Including this, the general multiplicity formula is

$$
\mathcal{M}_{[n-m, m], p}= \begin{cases}(5.58) & l(p) \leq N \\ 0 & l(p)>N\end{cases}
$$


We can also look at the total $\Lambda$ multiplicity $\mathcal{M}_{\Lambda}$ by summing over all $p \vdash n$

$$
\begin{aligned}
\mathcal{M}_{[n-m, m]}= & \sum_{p \vdash n, l(p) \leq N} \mathcal{M}_{[n-m, m], p} \\
= & \sum_{p \vdash n, l(p) \leq N}\left(\sum_{\lambda \vdash m}\left[\prod_{k}\left(s_{\lambda_{k}}-k+1\right)\right]\left[\prod_{i} \frac{1}{\mu_{i}(\lambda) !}\right]\right. \\
& \left.\quad-\sum_{\lambda \vdash m-1}\left[\prod_{k}\left(s_{\lambda_{k}}-k+1\right)\right]\left[\prod_{i} \frac{1}{\mu_{i}(\lambda) !}\right]\right)
\end{aligned}
$$

From representation theory considerations [40], the sectors with $\Lambda=[n]$ and $[n-1,1]$ do not undergo a step-change as we turn on the coupling constant. Therefore the weak coupling combinatorics of these sectors should match the free field combinatorics of section 2.2. A priori, the combinatorics should agree when considering the entire $\Lambda=[n]$ or $[n-1,1]$ sector. We find a stronger result: the combinatorics of the Young diagram label $R$ in (2.64) matches the partition $p$ of this section.

From (5.63), for $\Lambda=[n]$ the multiplicity of any given $p$ is 1 , while for $\Lambda=[n-1,1]$ recall that $s_{1}=c_{0,1}$ is the number of corners of $p$, so the multiplicity of $p$ is simply the number of corners subtract 1. As expected, these match (2.66) and (2.67) respectively and therefore

$$
C(R, R, \Lambda)=\mathcal{M}_{\Lambda, R}
$$

for $\Lambda=[n]$ and $[n-1,1]$.

\subsection{Hermite reciprocity and $p$-orbits of fixed $\mathcal{M}_{\Lambda, p}$}

There are collections of $p$ which lead to the same multiplicities for all $\Lambda$. To understand these, we look at the definition (5.29) of $F_{i, p_{i}}$. If $i>p_{i}$, the numerator and denominator start cancelling, and we end up with

$$
F_{i, p_{i}}=\frac{\left(1-q^{i+1}\right)}{(1-q)} \frac{\left(1-q^{i+2}\right)}{\left(1-q^{2}\right)} \cdots \frac{\left(1-q^{i+p_{i}}\right)}{\left(1-q^{p_{i}}\right)}=F_{p_{i}, i}
$$

We can rewrite this to be explicitly symmetric in $i \leftrightarrow p_{i}$

$$
F_{i, p_{i}}=F_{p_{i}, i}=\frac{\left(1-q^{\max \left(i, p_{i}\right)+1}\right)}{(1-q)} \frac{\left(1-q^{\max \left(i, p_{i}\right)+2}\right)}{\left(1-q^{2}\right)} \cdots \frac{\left(1-q^{i+p_{i}}\right)}{\left(1-q^{\min \left(i, p_{i}\right)}\right)}
$$

This symmetry is known as Hermite reciprocity [59] and can be viewed as a property of $\mathrm{SU}(2)$ characters.

We can use this $i \leftrightarrow p_{i}$ symmetry to do transformations on partitions that keep the product $F_{p}$ the same, and by extension all the associated $\Lambda$ multiplicities.

As our first example, take $p$ to be rectangular, so $p=\left[i^{p_{i}}\right]$ for some particular choice of $i$. Then the conjugate partition $p^{c}=\left[\left(p_{i}\right)^{i}\right]$ has the same $F$, leading to the same multiplicities for all $\Lambda$. Note that $p^{c}=p$ if $i=p_{i}$. 
Now suppose $p=\left[i^{p_{i}}, j^{p_{j}}\right]$ for $i<j$. Then there are three candidates for partitions with the same $F$, namely

$$
\begin{aligned}
& p^{(1)}=\left[\left(p_{i}\right)^{i}, j^{p_{j}}\right] \\
& p^{(2)}=\left[i^{p_{i}},\left(p_{j}\right)^{j}\right] \\
& p^{(3)}=\left[\left(p_{i}\right)^{i},\left(p_{j}\right)^{j}\right]
\end{aligned}
$$

The partition given by $p^{(1)}$ will only produce the same $F$ if $j \neq p_{i}$. If $j=p_{i}$, then $p^{(1)}$ should be written as $\left[j^{i+p_{j}}\right]$ and the $F$ s no longer match. Similarly $p^{(2)}$ will only match if $i \neq p_{j}$ and $p^{(3)}$ if $p_{i} \neq p_{j}$.

To visualise the transformations taking $p$ to $p^{(1,2,3)}$, split $p$ into two rectangles stacked on top of each other. Then $p^{(1)}$ is obtained by rotating the $i$ rectangle through 90 degrees, reordering the two rectangles if appropriate, and re-stacking them. In the same manner, $p^{(2)}$ is obtained by rotating the $j$ rectangle, and $p^{(3)}$ by rotating both. We take $p=[4,3,3]$ as an example

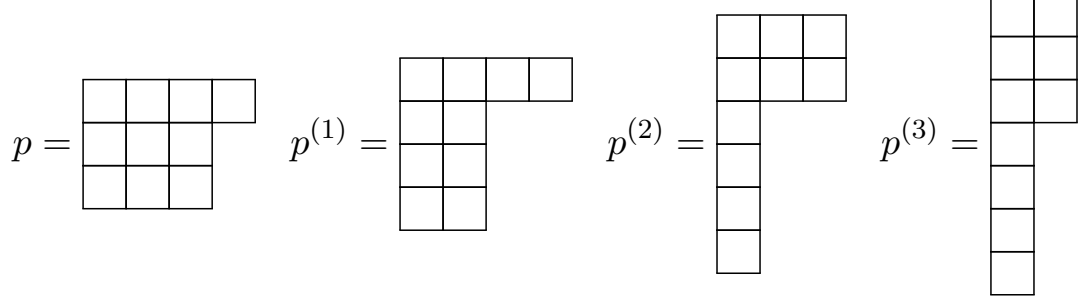

When one of the dimensions of the first rectangle coincides with one of the dimensions of the second rectangle, one or more of these four options will reduce from two distinct rectangles into one larger rectangle, and hence to a different $F$. If there is one coincidence, for example $p=[3,2]$ where we have $p_{2}=p_{3}=1$, we only have three partitions with the same $F$

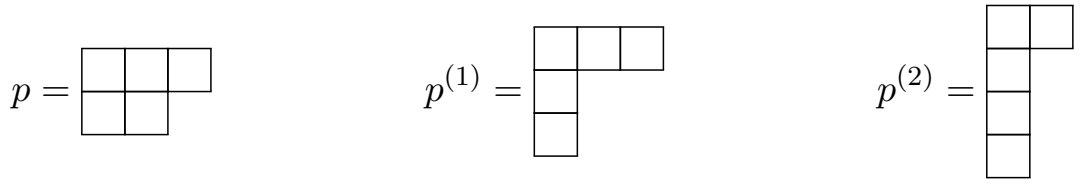

If there are two coincidences, then the partition is not related to any other via these transformations. There are three distinct ways for these two coincidences to occur. Firstly, three of the dimensions could be the same, while the fourth is different, for example $p=$ $[2,2,1,1]$. Secondly, both rectangles are squares, with distinct sizes, for example $p=$ $[2,2,1]$. Finally, the two rectangles are identical, but are non-square, for example $p=$ $[2,1,1]$. These three partitions are shown below
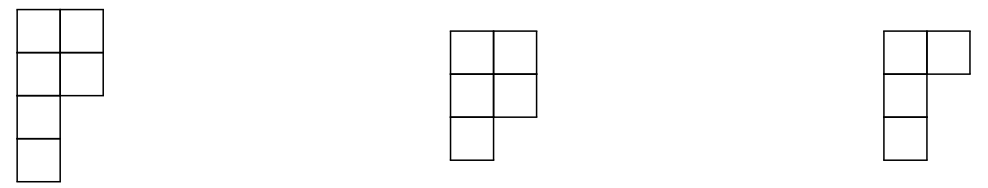

The generalisation to more rectangles is straightforward. A partition made from $k$ rectangles can be related to as many as $2^{k}$ others by rotating a subset of the $k$ rectangles. 
These rotations are only valid if the widths of all the rotated rectangles are distinct. As an example, consider all partitions of 5 . These fall into 4 orbits under these rotations

$$
\begin{array}{ll}
o_{1}=\{\square, \boxminus\} & o_{2}=\{\boxminus \square\} \\
o_{3}=\{\boxminus, \boxminus, \boxminus\} & o_{4}=\{\boxminus\}
\end{array}
$$

The equivalent classification for partitions of 6 is

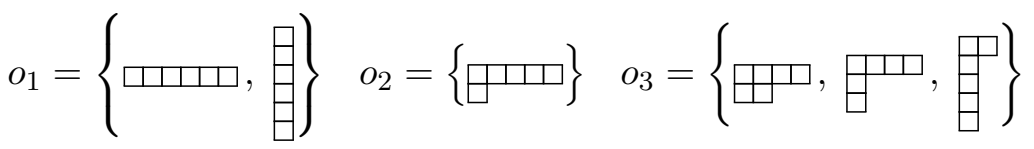

$$
\begin{aligned}
& o_{4}=\{\boxplus, \boxplus\} \quad o_{5}=\{\oplus\} \quad o_{6}=\{\boxminus \square\} \\
& o_{7}=\{\notin\}
\end{aligned}
$$

In appendices $\mathrm{A}, \mathrm{B}$ and $\mathrm{C}$, we give explicit formulae for the $n=5,6$ basis elements. As expected, the families of partitions above have the same multiplicities for all $\Lambda$.

The first orbit constructed from 2 rectangles to have all 4 dimensions distinct, and therefore achieve the maximum size of $4=2^{2}$ is found at $n=10$ and is shown in (5.72). The first orbit constructed from 3 rectangles to have all 6 dimensions distinct and have maximum size $8=2^{3}$ is found at $n=28$, and consists of

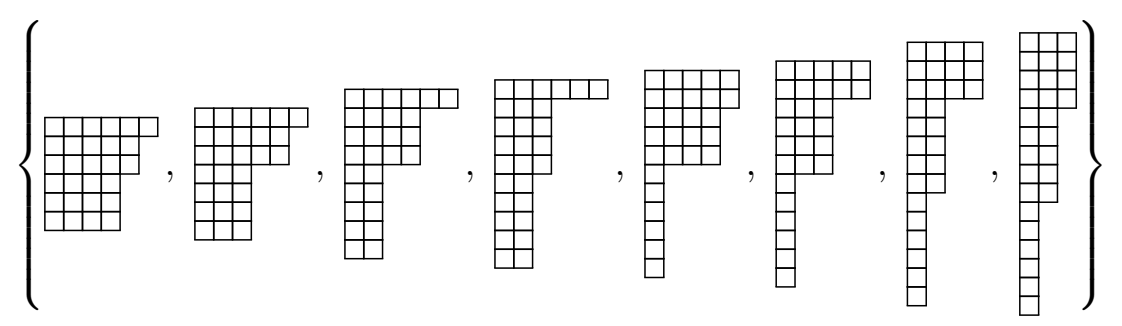

In the half-BPS sector, the Young diagram label of the Schur basis (2.18) gives us the properties of the corresponding giant graviton in the AdS/CFT correspondence. A partition with $k \sim 1$ rows of length $L \sim N$ describes $k$ giant gravitons (or a single giant graviton wrapped $k$ times) extended in $A d S_{5}$ of angular momentum $L$. Similarly, a partition with $k$ columns of length of length $L$ describes $k$ giant gravitons extended in $S^{5}$ of angular momentum $L$.

These orbits of partitions with identical multiplicities at all $\Lambda$ allow us to identify families of different giant graviton states that behave the same under quarter-BPS deformations.

Taking the simplest example of a single rectangle with $p=\left[k^{L}\right]$, this rectangle rotation symmetry means $k$ coincident giant gravitons rotating with angular momentum $L$ in $A d S_{5}$ behave the same way as $k$ coincident giant gravitons rotating with angular momentum $L$ in $S^{5}$. 
A system of two AdS giant gravitons with different angular momenta has $p=\left[k_{1}, k_{2}\right]$ with $k_{1}, k_{2} \sim N$ and $k_{1} \neq k_{2}$. Then we can rotate each of the rows individually to get $p=\left[k_{1}, 1^{k_{2}}\right]$ or $p=\left[k_{2}, 1^{k_{1}}\right]$, however we cannot rotate both at the same time. Therefore the behaviour of two non-coincident sphere giants under quarter-BPS deformations is different to that of two non-coincident AdS giants. This is studied further in the next section.

\subsection{Calculation of multiplicities for simplest orbits}

For some of the simplest orbits of partitions under rectangle rotation, we can describe the $\Lambda$ multiplicities explicitly. These are dual to one or two giant gravitons wrapped around the $A d S_{5}$ or $S^{5}$ factors of $A d S_{5} \times S^{5}$.

Recall from (5.33) that $(1-q) F_{p}$ is the generating function for the $\Lambda$ multiplicities. More specifically, the coefficient of $q^{m}$ is the multiplicity of $\Lambda=[n-m, m]$ for $m \leq\left\lfloor\frac{n}{2}\right\rfloor$.

We start with $p=\left[1^{n}\right]$ or equivalently $p=[n]$.

$$
(1-q) F_{[n]}=(1-q) F_{1, n}=1-q^{n+1}
$$

So $\Lambda=[n]$ appears with multiplicity 1 , and all other $\Lambda$ have multiplicity 0 . In the dual string theory, this means a single half-BPS giant graviton cannot deform into the quarterBPS sector.

We next consider rectangles with side lengths 2 and $k \geq 2$, so $p=\left[2^{k}\right]$ or $[k, k]$.

$$
\begin{aligned}
(1-q) F_{[k, k]} & =(1-q) F_{2, k}=\frac{\left(1-q^{k+1}\right)\left(1-q^{k+2}\right)}{1-q^{2}} \\
& =\left(1+q^{2}+q^{4}+\ldots\right)\left(1-q^{k+1}-q^{k+2}+q^{2 k+3}\right) \\
& =1+q^{2}+q^{4}+\cdots+q^{\lfloor k\rfloor_{2}}+O\left(q^{k+1}\right)
\end{aligned}
$$

where $\lfloor k\rfloor_{2}=2\left\lfloor\frac{k}{2}\right\rfloor$ is $k$ rounded down to the nearest multiple of 2 . Since we are only interested in the terms with exponent $\leq k=\frac{n}{2}$, we can ignore the $O\left(q^{k+1}\right)$ parts of the expression. Then $\Lambda=[n-m, m]$ appears with multiplicity 1 if $m$ is even, and 0 otherwise.

The dual interpretation of $p=[k, k]$ is two coincident AdS giants, while $p=\left[2^{k}\right]$ is two coincident sphere giants. Then (5.79) states that these states can be deformed deep into the quarter-BPS sector. In some sense, the quarter-BPS state 'furthest' from half-BPS is $\Lambda=\left[\frac{n}{2}, \frac{n}{2}\right]$, and this arrangement of giants can be deformed right up to that limit if $\frac{n}{2}$ is even (and only one away if $\frac{n}{2}$ odd). However, not all quarter-BPS deformations are available. In particular the 'smallest' deformation $\Lambda=[n-1,1]$ does not exist, and we must deform by 'twice' as much for each step into the quarter-BPS.

Now look at a combination of two rectangles, both with one dimension of length 1. Let the other dimensions be $k \geq l$. If $k=l$, then the orbit has size 1 , namely $p=\left[k, 1^{k}\right]$. Otherwise, the orbit consists of three partitions, $p=[k, l], p=\left[k, 1^{l}\right], p=\left[l, 1^{k}\right]$. The considerations of the orbit size do not affect the calculation of multiplicities. This 
calculation is

$$
\begin{aligned}
(1-q) F_{[k, l]} & =(1-q) F_{1, k} F_{1, l}=\frac{\left(1-q^{k+1}\right)\left(1-q^{l+1}\right)}{1-q} \\
& =\left(1+q+q^{2}+\ldots\right)\left(1-q^{l+1}-q^{k+1}+q^{k+l+2}\right) \\
& =1+q+q^{2}+\cdots+q^{l}+O\left(q^{k+1}\right)
\end{aligned}
$$

So $\Lambda=[n-m, m]$ appears with multiplicity 1 if $m \leq l$ and 0 otherwise.

For $k>l$, based on the argument that keeping $Y(p)$ fixed and deforming $\Lambda=[n]$ to $\Lambda=\left[n-n_{2}, n_{2}\right]$ preserves the qualitative physics of the giant states, we expect the partition $p=[k, l]$ corresponds to two non-coincident AdS giants when $k, l$ are of order $N$. The multiplicity of $\Lambda$ we are getting above is precisely the multiplicity of $\mathrm{U}(2)$ reps in $(\Lambda=[k]) \otimes(\Lambda=[l])$. Indeed the $\mathrm{U}(2)$ representation for the quantum states constructed from multi-symmetric functions $M_{\mathbf{p}}$ with associated partition $p=[k, l]$ is $\operatorname{Sym}^{k}\left(V_{2}\right) \otimes$ $\operatorname{Sym}^{l}\left(V_{2}\right)=\mathcal{R}_{p=[k]}^{\mathrm{U}(2)} \otimes \mathcal{R}_{p=[l]}^{\mathrm{U}(2)}$. The case $p=[k, k]$ corresponds, by the same argument, to bound state of two AdS giants of angular momentum $k$. In this case the multi-symmetric construction gives the $\mathrm{U}(2)$ representation $\operatorname{Sym}^{2}\left(\operatorname{Sym}^{k}\left(V_{2}\right)\right)=\operatorname{Sym}^{2}(\Lambda=[k])$, which is the symmetric subspace of $\mathcal{R}_{p=[k]}^{\mathrm{U}(2)} \otimes \mathcal{R}_{p=[k]}^{\mathrm{U}(2)}$. The projection to the symmetric part accounts for the missing powers of $q$ in (5.79) compared to the $p=[k, l]$ case (5.80).

To look at two non-coincident sphere giants, we consider $p=[l, l, k-l]$ for $k>l$. After rotating both rectangles, this is equivalent to $p=\left[2^{l}, 1^{k-l}\right]$, corresponding to two sphere giants of momenta $k$ and $l$ respectively. This has

$$
\begin{aligned}
(1-q) F_{[l, l, k-l]}= & (1-q) F_{2, l} F_{1, k-l}=\frac{\left(1-q^{l+1}\right)\left(1-q^{l+2}\right)\left(1-q^{k-l+1}\right)}{(1-q)\left(1-q^{2}\right)} \\
= & \left(1-q^{k-l+1}-q^{l+1}-q^{l+2}+q^{2 l+3}+O\left(q^{k}\right)\right) \\
& \left(1+q+2 q^{2}+2 q^{3}+3 q^{4}+3 q^{5}+\ldots\right)
\end{aligned}
$$

Where we can ignore terms of order $k$ and higher as these exponents are greater than $\frac{n}{2}=\frac{k+l}{2}$.

Let $a_{m}=0$ for $m<0$ and $a_{m}=\left\lfloor\frac{m}{2}\right\rfloor+1$ for $m \geq 0$, the coefficient of $q^{m}$ in the second factor of (5.81). Then the coefficient of $q^{m}$ in (5.81) is

$$
\mathcal{M}_{[n-m, m],[l, l, k-l]}=a_{m}-a_{m-k+l-1}-a_{m-l-1}-a_{m-l-2}+a_{m-2 l-3}
$$

The exact formulae for the multiplicities depend on the relative sizes of $k$ and $l$. If $k \leq 2 l$, then

$$
\mathcal{M}_{[n-m, m],[l, l, k-l]}= \begin{cases}\left\lfloor\frac{m}{2}\right\rfloor+1 & 0 \leq m \leq k-l \\ \left\lfloor\frac{m}{2}\right\rfloor-\left\lfloor\frac{m-k+l-1}{2}\right\rfloor & k-l+1 \leq m \leq l \\ \left\lfloor\frac{m}{2}\right\rfloor-\left\lfloor\frac{m-k+l-1}{2}\right\rfloor-m+l & l+1 \leq m \leq \frac{k+l}{2}\end{cases}
$$

where we have used

$$
\left\lfloor\frac{c-1}{2}\right\rfloor+\left\lfloor\frac{c-2}{2}\right\rfloor=\frac{c-1}{2}+\frac{c-2}{2}-\frac{1}{2}=c-2
$$


for $c=m-l$.

If $2 l \leq k \leq 3 l$, then

$$
\mathcal{M}_{[n-m, m],[l, l, k-l]}= \begin{cases}\left\lfloor\frac{m}{2}\right\rfloor+1 & 0 \leq m \leq l \\ \left\lfloor\frac{m}{2}\right\rfloor-m+l+1 & l+1 \leq m \leq k-l \\ \left\lfloor\frac{m}{2}\right\rfloor-\left\lfloor\frac{m-k+l-1}{2}\right\rfloor-m+l & k-l+1 \leq m \leq \frac{k+l}{2}\end{cases}
$$

Finally, if $k \geq 3 l$

$$
\mathcal{M}_{[n-m, m],[l, l, k-l]}= \begin{cases}\left\lfloor\frac{m}{2}\right\rfloor+1 & 0 \leq m \leq l \\ \left\lfloor\frac{m}{2}\right\rfloor-m+l+1 & l+1 \leq m \leq 2 l \\ 0 & 2 l+1 \leq m \leq \frac{k+l}{2}\end{cases}
$$

For two sphere giants of momenta $k, l \lesssim N$, the multiplicities fall into category (5.83). Roughly speaking, the multiplicity of $\Lambda=[n-m, m]$ increases as $\frac{m}{2}$ until reaching $\frac{k-l}{2}$. It then stays constant until $m$ reaches $l$ before turning around and decreasing for $m \geq l$, reaching 0 at $m=\frac{n}{2}$.

From the construction based on multi-symmetric functions, the states for $p=\left[2^{l}, 1^{k-l}\right]$ form, in all the cases, the $\mathrm{U}(2)$ representation

$$
\begin{aligned}
\operatorname{Sym}^{l}\left(\operatorname{Sym}^{2}\left(V_{2}\right)\right) \otimes \operatorname{Sym}^{k-l}\left(V_{2}\right) & =\operatorname{Sym}^{2}\left(\operatorname{Sym}^{l}\left(V_{2}\right)\right) \otimes \operatorname{Sym}^{k-l}\left(V_{2}\right) \\
& =\mathcal{R}_{p=\left[2^{l}\right]}^{\mathrm{U}(2)} \otimes \mathcal{R}_{p=\left[1^{k-l}\right]}^{\mathrm{U}(2)} \\
& =\mathcal{R}_{p=[l, l]}^{\mathrm{U}(2)} \otimes \mathcal{R}_{p=[k-l]}^{\mathrm{U}(2)}
\end{aligned}
$$

So the construction implies that the 2 -sphere-giant system for $p=\left[2^{k}, 1^{k-l}\right]$ have the same multiplicities as a composite consisting of the 2-sphere-giant bound state $p=\left[2^{l}\right]$ along with a 1 -sphere giant system $\left[1^{(k-l)}\right]$, while Hermite reciprocity further implies that these multiplicities are also the same as those of an AdS 2-giant bound state of angular momentum $l$ composed with a single AdS giant of angular momentum $k-l$.

We can see a marked difference between the behaviour of two non-coincident sphere giants compared to two non-coincident AdS giants. In (5.80) the multiplicity of each $\Lambda$ was at most 1 , so there was a unique way of deforming the arrangement of AdS giants at each stage on their way into the quarter-BPS sector. Furthermore, the furthest possible deformation was $m=l$, the lesser of the two momenta of the gravitons. With (5.83) the multiplicities can be larger than 1 , and are non-zero right up to $m=\frac{n}{2}$. So there are a multitude of ways of deforming sphere giants, and they can be deformed all the way into the quarter-BPS. Interestingly, when the two momenta are more uneven, and $m$ can get as high as $m=2 l$, there is a cut-off on the possible deformations. This is twice the equivalent cut-off for non-coincident AdS giants.

We have interpreted $p=\left[2^{l}, 1^{k-l}\right]$ as corresponding to two non-coincident sphere giants in order to compare with the equivalent system of AdS giants. However, when $l, k-l \sim N$, the rotation $p=[l, l, k-l]$ is exactly the system of two coincident AdS giants of momenta $l$ 
and a third giant of momenta $k-l$. So two separated sphere giants have the same behaviour as a system of three AdS giants.

It is worth remarking that there are important differences in how the same Hilbert spaces of $N$ free bosons in a harmonic oscillator are arrived at in the two problems of quantizing moduli spaces of sphere giants [17] and the moduli space of AdS giants [18]. In [17] quarter-BPS multi-giant systems are described by Fock space oscillators associated with higher order polynomials in $x, y$. In [18] there is a relatively simpler phase space of classical AdS solutions which is $\mathbb{C}^{2}$ (and $\mathbb{C}^{3}$ in the more general eighth-BPS case) and the full Hilbert space is obtained by considering an $N$ particle boson system based on this 1particle system. This serves to explain why the gauge theory construction of BPS operators we are giving here, which is intimately tied to a weak-coupling gauge theory realization of the multi-free boson Hilbert space, leads to simpler compositeness structures for the AdS giants as discussed above.

\subsection{Partitions with one dominant row or column}

There is another family of partitions that have nice properties. Consider $p \vdash n$ in which the first row dominates the partition, i.e. $p=\left[\lambda_{1}, \hat{p}\right]$, where $\lambda_{1} \geq \frac{n}{2}$ and $\hat{p} \vdash \hat{n}=n-\lambda_{1}$.

With one exception, when $\lambda_{1}=\frac{n}{2}$ and $\hat{p}=\left[\lambda_{1}\right]$ (this case has already been considered in $(5.79))$, this leads to

$$
F_{p}=F_{\lambda_{1}, 1} F_{\hat{p}}=\frac{1-q^{\lambda_{1}+1}}{1-q} F_{\hat{p}}
$$

and therefore

$$
(1-q) F_{p}=\left(1-q^{\lambda_{1}+1}\right) F_{\hat{p}}
$$

Using the second equation in (5.33), we have

$$
\begin{aligned}
\mathcal{M}_{[n-m, m],\left[\lambda_{1}, \hat{p}\right]} & =\operatorname{Coeff}\left[q^{m},(1-q) \prod_{i} F_{i, p_{i}}(q)\right] \\
& =\operatorname{Coeff}\left[q^{m},\left(1-q^{\lambda_{1}+1}\right) F_{\hat{p}}\right]
\end{aligned}
$$

Since $m \leq \frac{n}{2}$ for $\Lambda$ to be valid Young diagram and $\lambda_{1}+1>\frac{n}{2}$ by the dominant first row property, it follows that

$$
\operatorname{Coeff}\left(q^{m}, q^{\lambda_{1}+1} F_{\hat{p}}\right)=0
$$

and we can simplify (5.90) to

$$
\mathcal{M}_{[n-m, m],\left[\lambda_{1}, \hat{p}\right]}=\operatorname{Coeff}\left(q^{m}, F_{\hat{p}}\right)
$$

Thus, the generating function for the $\Lambda$ multiplicities is just $F_{\hat{p}}$ and does not depend on $\lambda_{1}$. We can now use our study of the coefficients of $F$ from section 5.3 to give the $\Lambda$ multiplicities. Note that the dominant first row condition has allowed us to obtain a simple formula for $(1-q) F_{p}$ in terms of $F_{\hat{p}}$. As a result the multiplicities are being obtained simply from the coefficients of a known generating function $\left(F_{\hat{p}}\right)$. This is simpler than the procedure of section 5.3 where the $\Lambda$ multiplicities were obtained from the difference of two consecutive coefficients in $F_{p}$. 
Using the formulae (5.59)-(5.63) we can write

$$
\begin{aligned}
F_{\hat{p}}(q)=\sum_{m} q^{m} \mathcal{M}_{[n-m, m],\left[\lambda_{1}, \hat{p}\right]}= & 1+s_{1} q+\left(s_{2}+\frac{s_{1}\left(s_{1}-1\right)}{2}\right) q^{2} \\
& +\left(s_{3}+s_{2}\left(s_{1}-1\right)+\frac{s_{1}\left(s_{1}-1\right)\left(s_{1}-2\right)}{6}\right) q^{3}+\ldots
\end{aligned}
$$

Where the $s_{i}$ refer to $s_{i}(\hat{p})$, not $s_{i}(p)$.

As previously observed in (5.57), the coefficients of $F_{\hat{p}}$ form a palindromic sequence, starting and ending with 1 at $q^{0}$ and $q^{\hat{n}}$. Adding this to (5.93), we have

$$
\begin{aligned}
\sum_{m} q^{m} \mathcal{M}_{[n-m, m],\left[\lambda_{1}, \hat{p}\right]}= & 1+s_{1} q+\left(s_{2}+\frac{s_{1}\left(s_{1}-1\right)}{2}\right) q^{2} \\
& +\left(s_{3}+s_{2}\left(s_{1}-1\right)+\frac{s_{1}\left(s_{1}-1\right)\left(s_{1}-2\right)}{6}\right) q^{3} \\
& +\ldots \\
& +\left(s_{3}+s_{2}\left(s_{1}-1\right)+\frac{s_{1}\left(s_{1}-1\right)\left(s_{1}-2\right)}{6}\right) q^{\hat{n}-3} \\
& +\left(s_{2}+\frac{s_{1}\left(s_{1}-1\right)}{2}\right) q^{\hat{n}-2}+s_{1} q^{\hat{n}-1}+q^{\hat{n}}
\end{aligned}
$$

In summary, for $p$ of the form $p=\left[\lambda_{1}, \hat{p}\right]$, where $\hat{p} \vdash \hat{n}$ and $\lambda_{1} \geq \frac{n}{2}$, the multiplicities of $\Lambda=[n]$ and $\Lambda=[n-\hat{n}, \hat{n}]$ are exactly 1 , the multiplicities of $\Lambda=[n-1,1]$ and $\Lambda=[n-\hat{n}+1, \hat{n}-1]$ are the number of corners in $\hat{p}$, and the general multiplicity of $\Lambda=[n-m, m]$ can be read from (5.56) with $p=\hat{p}$ if $m \leq \hat{n}$ or is 0 if $m>\hat{n}$. Furthermore, when $m \leq \hat{n}$, sending $m \rightarrow \hat{n}-m$ does not affect the multiplicity:

$$
\mathcal{M}_{[n-m, m],\left[\lambda_{1}, \hat{p}\right]}=\mathcal{M}_{[n-\hat{n}+m, \hat{n}-m],\left[\lambda_{1}, \hat{p}\right]}
$$

These properties have interesting implications. For a given $n, \hat{n} \leq \frac{n}{2}$, there are a large class of partitions with a dominant single row of length $n-\hat{n}$ for which the combinatorics of the deep quarter-BPS sector are determined by the combinatorics of the near half-BPS sector. For $\Lambda=[n-\hat{n}, \hat{n}]$, there is a multiplicity of exactly 1 for any of the partitions in this class, which is the same combinatorics as the half-BPS $\Lambda=[n]$. For $\Lambda=[n-\hat{n}+1, \hat{n}-1]$, the multiplicity is the same as the next to half-BPS $\Lambda=[n-1,1]$. For $\Lambda=[n]$ and $[n-1,1]$, there is no change in spectrum as we turn on the coupling constant. Therefore the combinatorics of the $\Lambda=[n-\hat{n}, \hat{n}],[n-\hat{n}+1, \hat{n}-1]$ sectors (for this class of partitions) at weak coupling are determined by free field considerations.

It would be interesting to find out whether this unreasonable effectiveness of the free theory has any connections to arguments in [20,60] that important features of black hole physics in $A d S_{5} \times S^{5}$ are captured by the free theory.

More generally, if $m \ll \hat{n} \lesssim \frac{n}{2}$, then $\Lambda=[n-m, m]$ is a small deviation from half-BPS while $\Lambda=[n-\hat{n}+m, \hat{n}-m]$ is a long way into the quarter-BPS sector, and yet their combinatorics are identical for this class of partitions. An interesting question is whether for these states with dominant first row in $p$ (or dominant first column) have a welldefined semi-classical brane or space-time interpretation which can explain the coincidence 
of multiplicities between near-half-BPS and far-into-quarter-BPS regimes. Near half-BPS states have been studied in the context of the BMN limit of AdS/CFT [61]. In the context of giant gravitons, the physics of perturbations, in some sense small, of well-separated multi-giants has been understood [62-64].

Using the rectangle rotation described in section 5.4, similar properties hold for a single large column. Consider $p$ with a first column of length $\mu_{1}$ and a partition $\bar{p}$ attached to the right. This is denoted by $p=\left[1^{\mu_{1}}\right]+\bar{p}$. In terms of rectangles we can use for the rotation symmetry, this is a partition $\left[1^{l(\bar{p})}\right]+\bar{p}$ with a single column below it of length $\mu_{1}-l(\bar{p})$. So setting $\lambda_{1}=\mu_{1}-l(\bar{p})$ and $\hat{p}=\left[1^{l(\bar{p})}\right]+\bar{p}, p$ is in the same rotation orbit as $\left[\lambda_{1}, \hat{p}\right]$, and we can apply the logic of this section directly to $p$.

As an example, consider $\mu_{1}=8$ and $\bar{p}=[2,1]$, with corresponding $\lambda_{1}=6, \hat{p}=[3,2]$. This is easiest to see visually

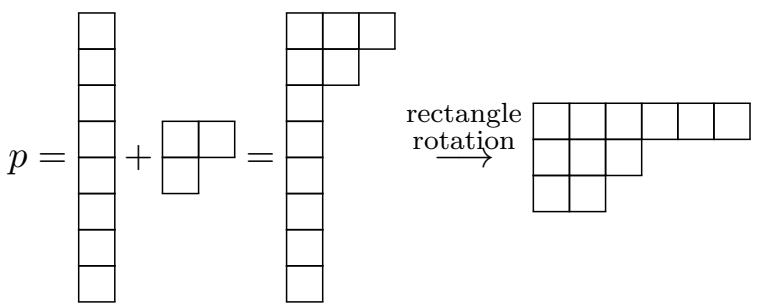

It is clear that the conditions on a single dominant column are more difficult to work with than those for a single dominant row. Let $\mu_{1}$ and $\mu_{2}$ be the length of the first and second columns respectively, then to use the analysis of this section, we require $\mu_{1}-\mu_{2} \geq \frac{n}{2}$. This is a far smaller class of diagrams than given by the analogous condition $\lambda_{1} \geq \frac{n}{2}$ for a diagram with a single dominant row.

\subsection{Identifying a multiplicity space basis}

In discussing the decomposition (5.9) we have not specified a choice of basis for $V_{\Lambda, p}^{\text {mult }}$, instead introducing a multiplicity index $\nu$ in the state (5.11). In this section we outline an algebraic approach to choosing a basis and characterising $\nu$.

As seen in (5.4), $R_{p}^{\mathrm{U}(2)}$ is a subspace of $\left(V_{2}\right)^{\otimes n}$. There is an $S_{n}$ action on $\left(V_{2}\right)^{\otimes n}$ by permutation of the tensor factors, and $R_{p}^{\mathrm{U}(2)}$ is the subspace invariant under the subgroup $G_{p}$ of $S_{n}$. For $p=<p_{1}, p_{2}, \cdots>\vdash n$, this subgroup, which is discussed in [32], is

$$
G_{p}=\underset{i}{\chi} S_{p_{i}}\left[S_{i}\right]
$$

where $S_{p_{i}}\left[S_{i}\right]$ is the wreath product of $S_{p_{i}}$ with $S_{i}$. This is defined as the semi-direct product of $S_{p_{i}}$ with $\left(S_{i}\right)^{p_{i}}$, where $S_{p_{i}}$ acts on $\left(S_{i}\right)^{p_{i}}$ by permutation of factors.

$G_{p}$ contains as a subgroup the group $G(\pi)$ given in (4.55), where $\pi \in \Pi(n)$ is a set partition with block size sizes given by $p$. $G_{p}$ consists of $G(\pi)$ with the addition of the $S_{p_{i}}$ factors.

The projector onto the $G_{p}$-invariant space is

$$
\mathbb{P}_{p}=\frac{1}{\left|G_{p}\right|} \sum_{\sigma \in G_{p}} \sigma
$$


which acts on a permutation $\tau \in S_{n}$ via the adjoint action

$$
\mathbb{P}_{p}(\tau)=\frac{1}{\left|G_{p}\right|} \sum_{\sigma \in G_{p}} \sigma \tau \sigma^{-1}
$$

This projector was used in [32] to derive the formula (5.37) for $\mathcal{M}_{\Lambda, p}$.

On the full space $\left(V_{2}\right)^{\otimes n}$, the $\mathrm{U}(2)$ and $S_{n}$ actions commute, and therefore they still commute on the $R_{p}^{\mathrm{U}(2)}$ subspace. Since $R_{p}^{\mathrm{U}(2)}$ is the $G_{p}$-invariant subspace, we should consider the action of the permutation subalgebra invariant under $G_{p}$-conjugation, rather than the full group algebra $\mathbb{C}\left(S_{n}\right)$. This algebra is

$$
\mathcal{A}_{p}=\mathbb{P}_{p}\left[\mathbb{C}\left(S_{n}\right)\right]=\left\{\alpha \in \mathbb{C}\left(S_{n}\right) \mid \sigma \alpha \sigma^{-1}=\alpha, \forall \sigma \in G_{p}\right\}
$$

Now $\mathcal{A}_{p}$ acts on $R_{p}^{\mathrm{U}(2)}$, but commutes with $\mathrm{U}(2)$, which means in the decomposition (5.9) it acts only on the multiplicity space components. So to choose a basis for $V_{\Lambda, p}^{\text {mult }}$, we can choose a maximally commuting set of operators in $\mathcal{A}_{p}$ and label the multiplicity space basis by the eigenvalues of these operators.

The algebras $\mathcal{A}_{p}$ are in general quite complicated, and finding a maximally commuting set of operators within them is an involved computational problem that we do not attempt to find a general solution for. They are a generalisation of the algebras studied in [30,65].

\section{Construction of orthogonal $\mathrm{U}(2) \times \mathrm{U}(N)$ Young-diagram-labelled basis}

In section 7 we show that for any $n, N$, we can construct BPS operators by applying $\mathcal{G}_{N}$ to the subspace $\overline{\mathcal{M}} \leq \mathbb{N} \subset \mathbb{C}\left(S_{n}\right)$, and using the map (2.63) from permutations to gauge invariant operators built from $N \times N$ matrices. The physical inner product on such operators obtained from 2-point functions uses the element $\mathcal{F}_{N}: \delta\left(\left(\mathcal{G}_{N} \sigma_{1}\right) \mathcal{F}_{N}\left(\mathcal{G}_{N} \sigma_{2}\right)\right)=\delta\left(\sigma_{1} \mathcal{G}_{N} \sigma_{2}\right)$. An orthogonal basis is obtained by choosing an ordering (section 6.1) on the labels of the basis elements of $\overline{\mathcal{M}} \leq$ and Gram-Schmidt orthogonalising. The orthogonal basis elements $S_{\Lambda, M_{\Lambda}, p, \nu}^{\mathrm{BPS}}$ are normalised in the $S_{n}$ inner product given in (2.70) (in section 7 this is the $g_{n, N}$ inner product). This construction algorithm gives a basis of BPS operators which is not only orthogonal but also SEP-compatible.

In this section we explain the construction of this orthogonal SEP-compatible basis of BPS operators from the covariant monomials $M_{\Lambda, M_{\Lambda}, p, \nu}$. We work with $N \times N$ matrices $X$ and $Y$, of which there are $n$ in total, where we can consider $N \geq n$ or $N<n$.

The final output will be a basis of BPS operators of the form

$$
S_{\Lambda, M_{\Lambda}, p, \nu}^{\mathrm{BPS}}=\sum_{\substack{R, \tau \\ l(R) \leq N}} s_{p, \nu}^{R, \tau}(\Lambda ; N) \mathcal{O}_{\Lambda, M_{\Lambda}, R, \tau}
$$

where $\mathcal{O}_{\Lambda, M_{\Lambda}, R, \tau}$ are the free field operators defined in (2.64) and the expansion coefficients $s_{p, \nu}^{R, \tau}(\Lambda ; N)$ are functions of $N$. These will in general consist of a polynomial numerator and a denominator that is the square root of a polynomial.

Let us give a precise statement of SEP-compatibility for these operators. Take some $\widehat{N} \leq N$, and evaluate these operators on matrices $X$ and $Y$ of size $\widehat{N} \times \widehat{N}$ instead of size 
$N \times N$. This means the free field operators with $l(R)>\widehat{N}$ vanish, and the coefficients are evaluated at $\widehat{N}$ rather than $N$. Then the operators with $l(p)>\widehat{N}$ will vanish and the operators with $l(p) \leq \widehat{N}$ will form a basis for the reduced BPS sector. Moreover, these are exactly the operators that would be produced by applying the construction algorithm directly with matrices of size $\widehat{N} \times \widehat{N}$.

Sections 6.1 through 6.4 describe how to construct $S_{\Lambda, M_{\Lambda}, p, \nu}^{\mathrm{BPS}}$ and prove that this basis is indeed BPS and SEP-compatible. In section 6.5 we give an equivalent, shorter construction that is represented in figure 2. The remaining sections investigate various properties of the bases.

\subsection{Orthogonalisation and SEP compatibility}

In order to construct the basis (6.1), we use results from section 7. Define

$$
\begin{aligned}
& \mathcal{M}_{\widehat{N}}^{>}(N)=\operatorname{Span}\left\{M_{\Lambda, M_{\Lambda}, p, \nu}(N): l(p)>\widehat{N}\right\} \\
& \mathcal{M} \underset{\widehat{N}}{\leq}(N)=\operatorname{Span}\left\{M_{\Lambda, M_{\Lambda}, p, \nu}(N): l(p) \leq \widehat{N}\right\}
\end{aligned}
$$

for some $\widehat{N} \leq N$. The operators $M_{\Lambda, M_{\Lambda}, p, \nu}(N)$ are constructed by employing the permu-

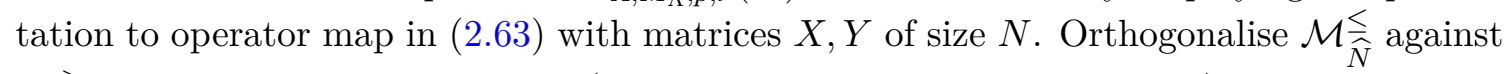
$\mathcal{M}_{\widehat{N}}$ using the $S_{n}$ inner product (the $g_{n, \widehat{N}}$ inner product in section 7 ), and denote the orthogonalised space by $\overline{\mathcal{M}} \leq \widehat{\widehat{N}}$. Note here the distinction between $N$ and $\widehat{N}$. The operators are defined using matrices of size $N \times N$, while $\widehat{N}$ is used to separate the operators into two classes depending on the length of $p$, the partition label for operators.

The result (7.59) and the discussion below it prove several useful facts.

1. Setting $\widehat{N}=N, \overline{\mathcal{M}} \leq$ is the entire pre-BPS sector.

2. The subspace $\overline{\mathcal{M}} \leq \frac{\leq}{\hat{N}}(N)$ is within the span of free field operators with $l(R) \leq \widehat{N}$. In particular, operators within $\overline{\mathcal{M}} \leq \widehat{\widehat{N}}$ do not receive any contribution from free field operators with $l(R)>\widehat{N}$. To see this, note that $\overline{\mathcal{M}} \leq \widehat{\widehat{N}}=\operatorname{Im}(\mathcal{P}) \cap \operatorname{Im}\left(\mathcal{F}_{\widehat{N}}\right)$. The general gauge invariant operators for matrices of size $N$ are constructed using permutation group algebra elements cut-off by $l(R) \leq N$. The definition of $\overline{\mathcal{M}} \leq \widehat{\widehat{N}}(N)$ involves the stronger restriction $l(R) \leq \widehat{N}$.

3. $\overline{\mathcal{M}} \leq \widehat{\widehat{N}}(N)$ gives a subspace of pre-BPS operators for matrices of size $N$. This subspace is such that, when we reduce $N$ to $\widehat{N}$ by lowering the size of the matrices $X$ and $Y$ to $\widehat{N} \times \widehat{N}$, these operators remain pre-BPS, and in fact form the entire pre-BPS sector.

The first of these results tells us the minimum work necessary to create BPS operators. Take an operator $M_{\Lambda, M_{\Lambda}, p, \nu}(N)$ with $l(p) \leq N$, and orthogonalise it against $\mathcal{M}_{N}^{>}$to give a new operator $\bar{M}_{\Lambda, M_{\Lambda}, p, \nu}(N)$. These form a basis for the quarter-BPS sector. If $N \geq n$, then $\mathcal{M}_{N}^{>}$is empty, and no orthogonalisation is necessary.

However, for $\widehat{N}<N$, operators $\bar{M}_{\Lambda, M_{\Lambda}, p, \nu}(N)$ with $l(p) \leq \widehat{N}$ are not necessarily orthogonal to $\mathcal{M}_{\widehat{N}}^{>}$, and therefore upon lowering $N$ to $\widehat{N}<N$, these are no longer preBPS. In other words, this is not an SEP-compatible basis, and more work is needed to find 
one. From the second and third points above, we have a sequence of pre-BPS spaces

$$
\overline{\mathcal{M}}_{1}^{\leq}(N) \subset \cdots \subset \overline{\mathcal{M}}_{N-1}^{\leq}(N) \subset \overline{\mathcal{M}}_{N}^{\leq}(N)
$$

such that for any $\widehat{N} \leq N$, the corresponding subspace $\overline{\mathcal{M}} \leq \widehat{\widehat{N}}$ is the entire pre-BPS sector when we lower $N$ to $\widehat{N}$.

It is now clear how we construct an SEP-compatible basis. Each operator $M_{\Lambda, M_{\Lambda}, p, \nu}(N)$ must be orthogonalised in the $S_{n}$ inner product against all operators $M_{\Lambda, M_{\Lambda}, q, \eta}(N)$ with $l(q)>l(p)$. Then for any $\widehat{N}$, the orthogonalised operators with $l(p) \leq \widehat{N}$ form a basis for

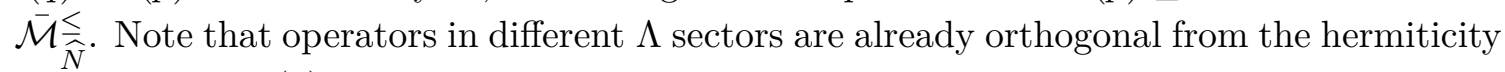
properties of $\mathrm{U}(2)$, so we do not need to consider these. In the subsequent discussion we will describe in more detail the steps involved in the construction of SEP-compatible orthogonal BPS operators starting from $M_{\Lambda, M_{\Lambda}, p, \nu}(N)$. We will henceforth drop the label $N$ and simply write $M_{\Lambda, M_{\Lambda}, p, \nu}$, with the fact that we are describing the construction for matrices of size $N$ being understood. The parameter $\widehat{N}<N$ will come up in discussion of SEP compatibility of the construction.

In section 3.2, we explained that if $N<n$, then $M_{\Lambda, M_{\Lambda}, p, \nu}$ picked an operator that differed from a pre-BPS operator by addition of a commutator trace. Intuitively, the orthogonalisation is removing the commutator trace part to leave only the pre-BPS operator.

Before implementing the orthogonalisation, recall from section 3.1 that for $N \geq n$, applying $\mathcal{G}_{N}$ to any basis of symmetrised traces gives BPS operators, without any complicated orthogonalisation procedure. From the above, we can give a weaker bound on $N$ within a specific $\Lambda$ sector.

Let $p_{\Lambda}^{*}$ be the longest (largest in the ordering (6.6)) partition with $\mathcal{M}_{\Lambda, p_{\Lambda}^{*}}>0$. In section 6.8 we prove that for $\Lambda=\left[\Lambda_{1}, \Lambda_{2}\right]$, we have

$$
l\left(p_{\Lambda}^{*}\right)=n-\left\lceil\frac{\Lambda_{2}}{2}\right\rceil
$$

Then if $N \geq l\left(p_{\Lambda}^{*}\right)$, then $\mathcal{M}_{N}^{>}$has no operators transforming on the $\Lambda$ representation of $\mathrm{U}(2)$ and the operators $M_{\Lambda, M_{\Lambda}, p, \nu}$ do not need to be orthogonalised before applying $\mathcal{G}_{N}$ to get BPS operators.

Returning to the construction, to carry out the procedure we will use Gram-Schmidt orthogonalisation, which requires choosing an ordering on partitions. To ensure the correct properties when we lower $N$ to some $\widehat{N} \leq N$, we must begin with the longest partition, and proceed to the shortest. For those with the same length, any ordering would suffice to create an SEP-compatible basis. A natural choice is to compare the length of the second column and start with the longer. If this is also the same length, the comparisons proceed along the columns until one is longer. If the partitions are the same and the operators occupy a multiplicity space, we do not specify an ordering. Any will suffice.

More formally, this ordering on partitions is the conjugate of the standard lexicographic ordering of partitions, which compares partitions based on the length of the first row, 
followed by the second row, etc. Given $p^{c}=\left[\lambda_{1}, \lambda_{2}, \ldots\right], q^{c}=\left[\mu_{1}, \mu_{2}, \ldots\right]$, then

$$
p>q \Longleftrightarrow \text { there is a } k \geq 1 \text { such that }\left\{\begin{array}{l}
\lambda_{i}=\mu_{i} \quad i<k \\
\lambda_{k}>\mu_{k}
\end{array}\right.
$$

The operators obtained by performing the orthogonalisation are denoted by $\bar{Q}_{\Lambda, M_{\Lambda}, p, \mu}$.

In section 6.8 we prove that $\mathcal{M}_{\Lambda, p_{\Lambda}^{*}}=1$, so we drop the multiplicity. For this partition we have

$$
\bar{Q}_{\Lambda, M_{\Lambda}, p_{\Lambda}^{*}}=M_{\Lambda, M_{\Lambda}, p_{\Lambda}^{*}}
$$

If we are performing the algorithm with $N<l\left(p_{\Lambda}^{*}\right)$, then the associated operator $M_{\Lambda, M_{\Lambda}, p_{\Lambda}^{*}}$ will reduce to a commutator trace or vanish. In the former case, there is no difference to the algorithm, while in the latter, we instead start the orthogonalisation with the largest $p$ such that $\mathcal{M}_{\Lambda, p}>0$ and the associated operator does not vanish. This partition will not necessarily have multiplicity 1 .

For the remaining $p, \nu$ the orthogonalised operators are defined inductively

$$
\bar{Q}_{\Lambda, M_{\Lambda}, p, \nu}=M_{\Lambda, M_{\Lambda}, p, \nu}-\sum_{(q, \eta)>(p, \nu)} \frac{\left\langle M_{\Lambda, M_{\Lambda}, p, \nu}, \bar{Q}_{\Lambda, M_{\Lambda}, q, \eta}\right\rangle_{S_{n}}}{\left\langle\bar{Q}_{\Lambda, M_{\Lambda}, q, \eta}, \bar{Q}_{\Lambda, M_{\Lambda}, q, \eta}\right\rangle_{S_{n}}} \bar{Q}_{\Lambda, M_{\Lambda}, q, \eta}
$$

where by $(q, \eta)>(p, \nu)$ we mean either $q>p$ in the ordering (6.6) or $q=p$ and $\eta>\nu$.

Similarly to the first step, it may occur that $\bar{Q}_{\Lambda, M_{\Lambda}, p, \nu}=0$ for some operators with $l(p)>N$. Such operators are excluded from the rest of the orthogonalisation algorithm. It is implicit that the sum in (6.8) does not run over these values of $q, \eta$.

In order to compare the different $\bar{Q}_{\Lambda, M_{\Lambda}, p, \nu}$, we normalise to have $S_{n}$ norm 1

$$
Q_{\Lambda, M_{\Lambda}, p, \nu}=\frac{\bar{Q}_{\Lambda, M_{\Lambda}, p, \nu}}{\sqrt{\left\langle\bar{Q}_{\Lambda, M_{\Lambda}, p, \nu} \mid \bar{Q}_{\Lambda, M_{\Lambda}, p, \nu}\right\rangle_{S_{n}}}}
$$

The new operators $Q_{\Lambda, M_{\Lambda}, p \nu}$ are an SEP-compatible basis for pre-BPS operators. They are orthonormal under the $S_{n}$ inner product, and form a stepping stone on the way to producing (6.1).

From the arguments above, we know that when expanding $Q_{\Lambda, M_{\Lambda}, p, \nu}$ in terms of the free field BPS operators (2.64), only those operators with $l(R) \leq l(p)$ contribute.

\subsection{An example: field content $(2,2)$}

We now give an explicit example of this construction for the field content $(2,2)$ sector at $N \geq n=4$. While doing so, we will observe various features that generalise to higher orders. These are discussed in later subsections.

We begin with the operators $M_{\Lambda, M_{\Lambda}, p, \nu}$ given in (5.14)-(5.22). The ordering (6.6) for partitions of $n=4$ is

$$
[1,1,1,1]>[2,1,1]>[2,2]>[3,1,1]>[4]
$$




\subsection{1 $\Lambda=[4]$ and $[3,1]$ sectors}

Orthogonalising in the $\Lambda=$ [4] sector, and normalising with respect to the $S_{n}$ inner product, we obtain

$$
\begin{aligned}
& Q_{[1|1| 2 \mid 2,[1,1,1,1]}=\frac{1}{4 \sqrt{6}}\left[-4 \operatorname{Tr} X^{2} Y^{2}-2 \operatorname{Tr}(X Y)^{2}+4 \operatorname{Tr} X^{2} Y \operatorname{Tr} Y+4 \operatorname{Tr} X \operatorname{Tr} X Y^{2}\right. \\
& +2(\operatorname{Tr} X Y)^{2}+\operatorname{Tr} X^{2} \operatorname{Tr} Y^{2}-\operatorname{Tr} X^{2}(\operatorname{Tr} Y)^{2} \\
& \left.-4 \operatorname{Tr} X \operatorname{Tr} X Y \operatorname{Tr} Y-(\operatorname{Tr} X)^{2} \operatorname{Tr} Y^{2}+(\operatorname{Tr} X)^{2}(\operatorname{Tr} Y)^{2}\right] \\
& Q_{[1|1| 2 \mid 2,[2,1,1]}=\frac{1}{4 \sqrt{6}}\left[4 \operatorname{Tr} X^{2} Y^{2}+2 \operatorname{Tr}(X Y)^{2}-2(\operatorname{Tr} X Y)^{2}-\operatorname{Tr} X^{2} \operatorname{Tr} Y^{2}\right. \\
& -\operatorname{Tr} X^{2}(\operatorname{Tr} Y)^{2}-4 \operatorname{Tr} X \operatorname{Tr} X Y \operatorname{Tr} Y-(\operatorname{Tr} X)^{2} \operatorname{Tr} Y^{2} \\
& \left.+3(\operatorname{Tr} X)^{2}(\operatorname{Tr} Y)^{2}\right]
\end{aligned}
$$

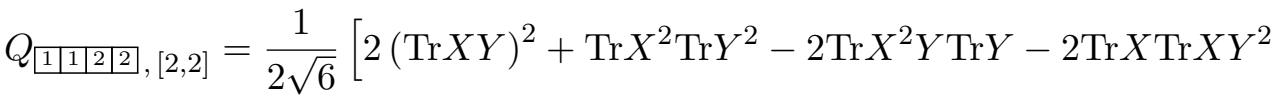

$$
\begin{aligned}
& \left.+(\operatorname{Tr} X)^{2}(\operatorname{Tr} Y)^{2}\right] \\
& Q_{[1|1| 2 \mid 2,[3,1]}=\frac{1}{4 \sqrt{6}}\left[-4 \operatorname{Tr} X^{2} Y^{2}-2 \operatorname{Tr}(X Y)^{2}-2(\operatorname{Tr} X Y)^{2}-\operatorname{Tr} X^{2} \operatorname{Tr} Y^{2}\right. \\
& +\operatorname{Tr} X^{2}(\operatorname{Tr} Y)^{2}+4 \operatorname{Tr} X \operatorname{Tr} X Y \operatorname{Tr} Y+(\operatorname{Tr} X)^{2} \operatorname{Tr} Y^{2} \\
& \left.+3(\operatorname{Tr} X)^{2}(\operatorname{Tr} Y)^{2}\right]
\end{aligned}
$$

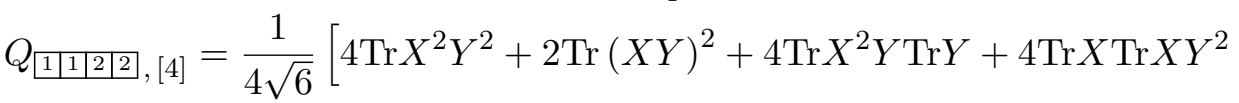

$$
\begin{aligned}
& +2(\operatorname{Tr} X Y)^{2}+\operatorname{Tr} X^{2} \operatorname{Tr} Y^{2}+(\operatorname{Tr} X)^{2}(\operatorname{Tr} Y)^{2} \\
& \left.+\operatorname{Tr} X^{2}(\operatorname{Tr} Y)^{2}+4 \operatorname{Tr} X \operatorname{Tr} X Y \operatorname{Tr} Y+(\operatorname{Tr} X)^{2} \operatorname{Tr} Y^{2}\right]
\end{aligned}
$$

where we have suppressed the trivial multiplicity indices, and omitted $\Lambda$ as this is determined by the shape of the semi-standard tableau $M_{\Lambda}$.

For $\Lambda=[3,1]$, the orthogonalisation process produces

$$
\begin{gathered}
Q_{\frac{11 \mid 2,[2,1,1]}{2}}=\frac{1}{4}\left[-2 \operatorname{Tr} X^{2} Y \operatorname{Tr} Y+2 \operatorname{Tr} X \operatorname{Tr} X Y^{2}+\operatorname{Tr} X^{2}(\operatorname{Tr} Y)^{2}-(\operatorname{Tr} X)^{2} \operatorname{Tr} Y^{2}\right] \\
Q_{\frac{1|1| 2,[3,1]}{2}}=\frac{1}{4}\left[2 \operatorname{Tr} X^{2} Y \operatorname{Tr} Y-2 \operatorname{Tr} X \operatorname{Tr} X Y^{2}+\operatorname{Tr} X^{2}(\operatorname{Tr} Y)^{2}-(\operatorname{Tr} X)^{2} \operatorname{Tr} Y^{2}\right]
\end{gathered}
$$

For both these sectors, the operators obtained are identical to the free field BPS operators, given for field content $(2,2)$ in table 1 , where the weak coupling label $p$ matches the zero coupling label $R$. At $n=4$, this is largely pre-determined by SEP-compatibility of the two bases.

Since the $\Lambda=[4]$ and $[3,1]$ sectors remain unchanged as we turn on interactions, the free field BPS operators are also the weak coupling BPS operators. As they are also eigenoperators of $\mathcal{F}_{N}$, the space of free field BPS operators is the same as the space of pre-BPS operators. Moreover, in these sectors there are no commutator traces, and therefore SEPcompatibility for the pre-BPS operators is the same as that for BPS operators, an operator with $l(p)>N$ vanishes identically. 
Therefore for $\Lambda=[4]$ and $[3,1], Q_{\Lambda, M_{\Lambda}, p, \nu}$ and $\mathcal{O}_{\Lambda, M_{\Lambda}, R, \tau}$ are both SEP-compatible bases for the same space. This means the spaces spanned by partitions of a given length are the same. These spaces are all one-dimensional with the exception of $\Lambda=[4], p=[2,2]$ and $p=[3,1]$. Therefore the matching of all but these two is trivial. A priori, the matching between $p$ and $R$ for $p=[3,1]$ and $[2,2]$ is surprising.

More generally, we find the same behaviour for all $n \leq 7$, which are within reach of numerical calculations. The $p$ label in $Q_{\Lambda, M_{\Lambda}, p, \nu}$ matches the $R$ label in $\mathcal{O}_{\Lambda, M_{\Lambda}, R, \tau}$ for $\Lambda=[n]$ and $[n-1,1]$. Since neither basis specifies exactly how the multiplicities $\nu$ and $\tau$ are chosen, these will not necessarily match, but they do span the same space. We go into this in more detail in section 6.9. For $\Lambda=[n]$, the matching follows from the fact the Kostka numbers converting the monomial basis to the Schur basis (2.16) are upper diagonal in partition indices. For $\Lambda=[n-1,1]$ we leave the matching at general $n$ as a conjecture.

\subsection{2 $\Lambda=[2,2]$ sector}

The orthogonalised basis of pre-BPS operators for $\Lambda=[2,2]$ is

$$
\begin{gathered}
Q_{\frac{11}{2 \frac{1}{2} 2},[2,1,1]}=\frac{1}{6}\left(-2 t_{[2,2]}+t_{[2,1,1]}\right) \\
Q_{\frac{11}{2 \frac{1}{2}},[2,2]}=\frac{1}{3 \sqrt{2}}\left(t_{[2,2]}+t_{[2,1,1]}\right)
\end{gathered}
$$

where the trace combinations are

$$
\begin{aligned}
t_{[2,1,1]} & =\operatorname{Tr} X^{2}(\operatorname{Tr} Y)^{2}-2 \operatorname{Tr} X \operatorname{Tr} X Y \operatorname{Tr} Y+(\operatorname{Tr} X)^{2} \operatorname{Tr} Y^{2} \\
t_{[2,2]} & =\operatorname{Tr} X^{2} \operatorname{Tr} Y^{2}-(\operatorname{Tr} X Y)^{2}
\end{aligned}
$$

These trace combinations are the $\Lambda=[2,2], M_{\Lambda}=\frac{1}{\frac{1}{2}} \frac{1}{2}$ parts of the symmetrised trace covariant basis defined in (5.36).

In order to check the SEP-compatibility of (6.18) and (6.19), we express them as a linear combination of the zero coupling basis given in table 1 . We have

$$
\begin{aligned}
& Q_{\frac{111}{22},[2,1,1]}=-\frac{1}{2 \sqrt{3}}\left(\mathcal{O}_{\frac{111}{22}}, \square \square+\sqrt{2} \mathcal{O}_{\frac{111}{222}}, \square+3 \mathcal{O}_{\frac{111}{22},}, \square\right)
\end{aligned}
$$

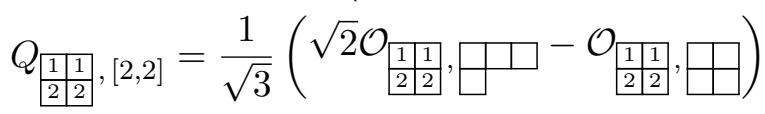

The only commutator trace at field content $(2,2)$ is

$$
\begin{aligned}
& \operatorname{Tr} X[X, Y] Y=\operatorname{Tr} X^{2} Y^{2}-\operatorname{Tr}(X Y)^{2} \\
& =\frac{\sqrt{3}}{2}\left(\mathcal{O}_{\frac{111}{22},}, \square \square+\sqrt{2} \mathcal{O}_{\frac{111}{22}}, \square-\mathcal{O}_{\frac{11}{2 \frac{1}{2}},}, \square\right)
\end{aligned}
$$

It is simple to check that for $N \geq 3$

$$
\left\langle Q_{\frac{1 \frac{1}{2 \frac{1}{2}}}{2},[2,1,1]} \mid \operatorname{Tr} X[X, Y] Y\right\rangle_{S_{n}}=\left\langle Q_{\frac{\sqrt{\frac{1}{2 \sqrt{2}}}}{2},[2,2]} \mid \operatorname{Tr} X[X, Y] Y\right\rangle_{S_{n}}=0
$$


At $N=2$, comparing (6.22) with (6.24) and recalling that $\mathcal{O}_{\frac{11}{22},}, \square$ vanishes, we have

$$
Q_{\frac{11 \frac{1}{22},[2,1,1]}{3}=-\frac{1}{3} \operatorname{Tr} X[X, Y] Y}
$$

Therefore (6.22) is no longer pre-BPS. The other operator (6.23) is still orthogonal to the commutator.

At $N=1$, both (6.22) and (6.23) vanish identically. Combined with the behaviour at $N=2$, this demonstrates that these two operators form an SEP-compatible basis for pre-BPS operators in the $\Lambda=[2,2]$ sector.

At $N=2$, the finite $N$ relations mean that (6.22) can be written as both a symmetrised trace and a commutator trace. This is discussed in more generality in section 7 , where we develop the finite $N$ vector space geometry responsible for transforming between the two types of traces.

\subsection{Normalisation conventions for BPS operators}

The final step to obtain an SEP-compatible basis for weakly coupled BPS operators is to apply $\mathcal{G}_{N}$ to $Q_{\Lambda, M_{\Lambda}, p, \nu}$, where $l(p) \leq N$. However, the operators $\mathcal{G}_{N} Q_{\Lambda, M_{\Lambda}, p, \nu}$ contain denominators of the form $(N-i)$ for $i \leq l(p)$ which make it difficult to see how they should behave when we lower $N$ to $\widehat{N}=i$.

In this section we prove that by normalising $\mathcal{G}_{N} Q_{\Lambda, M_{\Lambda}, p, \nu}$ under the $S_{n}$ inner product, we remove these denominators and obtain an SEP-compatible basis of BPS operators. We start by continuing the example of $\Lambda=[2,2]$ from the previous subsection to show some of the behaviour that occurs. Working at $N \geq 3$, we have

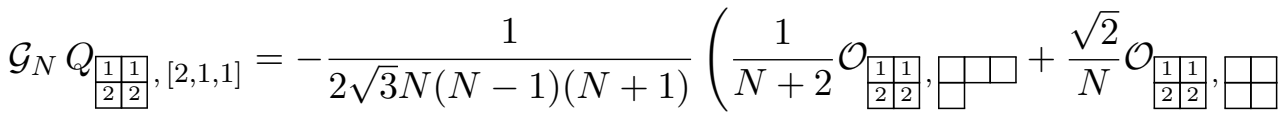

$$
\begin{aligned}
& \left.+\frac{3}{N-2} \mathcal{O}_{\frac{11}{2 \frac{1}{2}},}, \square\right)
\end{aligned}
$$

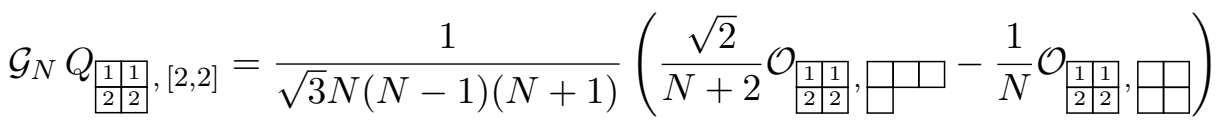

Now consider lower $N$ to $\widehat{N}=2$ and imposing the finite $\widehat{N}$ cut-off. It is unclear how we

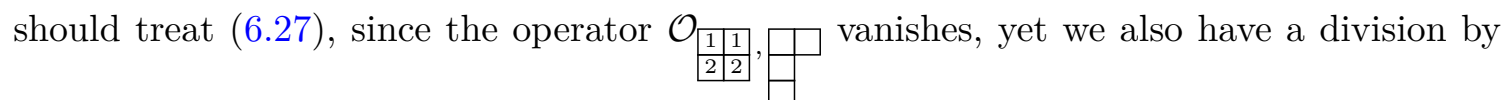
zero. Let us resolve the ambiguity by declaring that this term should indeed vanish. Then at $\widehat{N}=2$ we have

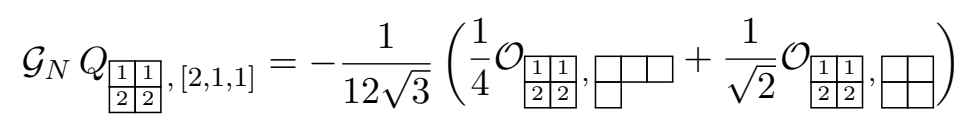

This is a perfectly well defined operator, yet $\mathcal{G}_{N} Q_{\Lambda, M_{\Lambda}, p, \nu}$ was meant to be an SEPcompatible basis. For BPS operators, this means (6.27) should vanish after reducing $N$ to $\widehat{N}=2$. 
The resolution of this problem is to normalise $\mathcal{G}_{N} Q_{\Lambda, M_{\Lambda}, p, \nu}$ in the $S_{n}$ inner product. Define

$$
Q_{\Lambda, M_{\Lambda}, p, \nu}^{\mathrm{BPS}}=\frac{\mathcal{G}_{N} Q_{\Lambda, M_{\Lambda}, p, \nu}}{\sqrt{\left\langle\mathcal{G}_{N} Q_{\Lambda, M_{\Lambda}, p, \nu} \mid \mathcal{G}_{N} Q_{\Lambda, M_{\Lambda}, p, \nu}\right\rangle_{S_{n}}}}
$$

For $\Lambda=[2,2]$ we have

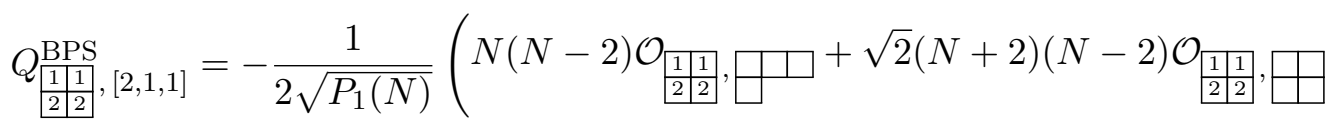

$$
\begin{aligned}
& \left.+3 N(N+2) \mathcal{O}_{\left.\frac{111}{2 \frac{1}{2}}, \square\right)}, \square\right)
\end{aligned}
$$

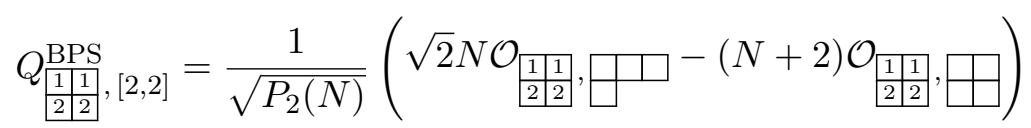

where the normalisation polynomials are

$$
\begin{aligned}
& P_{1}(N)=3 N^{4}+8 N^{3}+6 N^{2}+8 \\
& P_{2}(N)=3 N^{2}+4 N+4
\end{aligned}
$$

There is now no ambiguity in the definitions of the operators after lowering $N$ to $\widehat{N}=1,2$, and they vanish identically for $\widehat{N}<l(p)$, thereby forming an SEP-compatible basis for BPS operators.

We now generalise to arbitrary $\Lambda$. Take some operator $Q_{\Lambda, M_{\Lambda}, p, \nu}$ with $l(p) \leq N$ and expand it in terms of free field operators

$$
Q_{\Lambda, M_{\Lambda}, p, \nu}=\sum_{\substack{R, \tau \\ l(R) \leq l(p)}} q_{p, \nu}^{R, \tau} \mathcal{O}_{\Lambda, M_{\Lambda}, R, \tau}
$$

for some coefficients $q_{p, \nu}^{R, \tau}$. The limit $l(R) \leq l(p)$ on the sum was discussed below (6.9). Define the set

$$
Y_{p, \nu}=\left\{R: q_{p, \nu}^{R, \tau} \neq 0 \text { for some } \tau\right\}
$$

Intuitively, $Y_{p, \nu}$ is the set of Young diagrams $R$ that contribute to $Q_{\Lambda, M_{\lambda}, p, \nu}$. Define $R_{p, \nu}^{\max }$ to be the minimal Young diagram that contains every $R$ in $Y_{p, \nu}$ and $R_{p, \nu}^{\min }$ to be the maximal Young diagram that is contained within every $R$ in $Y_{p, \nu}$. Define

$$
f_{p, \nu}^{\max }(N)=f_{R_{p, \nu}^{\max }}(N) \quad f_{p, \nu}^{\min }(N)=f_{R_{p, \nu}^{\min }}(N)
$$

where $f_{R}(N)$ is a polynomial in $N$ depending on the shape of $R$. It is defined in (2.29) as a product of linear factors. Intuitively, $f_{p, \nu}^{\max }(N)$ is the lowest common multiple of the different $f_{R}$ for $R \in Y_{p, \nu}$, while $f_{p, \nu}^{\min }(N)$ is the highest common factor.

As an example, consider the previous example with $\Lambda=[2,2]$ and $p=[2,1,1]$. We have

$$
Y_{[2,1,1]}=\{\square, \square, \square\}
$$


and

$$
\begin{array}{ll}
R_{[2,1,1]}^{\max }=\square & f_{[2,1,1]}^{\max }(N)=(N+2)(N+1) N^{2}(N-1)(N-2) \\
R_{[2,1,1]}^{\min }=\square & f_{[2,1,1]}^{\min }(N)=(N+1) N(N-1)
\end{array}
$$

Using $f_{p, \nu}^{\max }(N)$, we can factor out the denominators in $\mathcal{G}_{N} Q_{\Lambda, M_{\Lambda}, p, \nu}$. We have

$$
\mathcal{G}_{N} Q_{\Lambda, M_{\Lambda}, p, \nu}=\sum_{\substack{R, \tau \\ l(R) \leq l(p)}} \frac{q_{p, \nu}^{R, \tau}}{f_{R}(N)} \mathcal{O}_{\Lambda, M_{\Lambda}, R, \tau}=\frac{1}{f_{p, \nu}^{\max }(N)} \sum_{\substack{R, \tau \\ l(R) \leq l(p)}} q_{p, \nu}^{R, \tau} \frac{f_{p, \nu}^{\max }(N)}{f_{R}(N)} \mathcal{O}_{\Lambda, M_{\Lambda}, R, \tau}
$$

where the coefficients $\frac{f_{p, \nu}^{\max }(N)}{f_{R}(N)}$ are simple polynomials in $N$ made up of products of linear factors. Therefore the $S_{n}$ norm of $\mathcal{G}_{N} Q_{\Lambda, M_{\Lambda}, p, \nu}$ is

$$
\left|\mathcal{G}_{N} Q_{\Lambda, M_{\Lambda}, p, \nu}\right|_{S_{n}}^{2}=\left\langle\mathcal{G}_{N} Q_{\Lambda, M_{\Lambda}, p, \nu} \mid \mathcal{G}_{N} Q_{\Lambda, M_{\Lambda}, p, \nu}\right\rangle_{S_{n}}=\frac{P_{p, \nu}(N)}{\left[f_{p, \nu}^{\max }(N)\right]^{2}}
$$

where the numerator polynomial is

$$
P_{p, \nu}(N)=\sum_{\substack{R, \tau \\ l(R) \leq l(p)}}\left(q_{p, \nu}^{R, \tau} \frac{f_{p, \nu}^{\max }(N)}{f_{R}(N)}\right)^{2}
$$

This polynomial is a sum of squares, and therefore can only vanish if all terms are zero. Since $\frac{f_{p, \nu}^{\max }(N)}{f_{R}(N)}$ is a product of simple linear factors in $N$, this in turn can only occur if there is linear factor common to every term. From the definition of $f_{p, \nu}^{\max }$ as the lowest common multiple of the $f_{R}$ that appear in the sum, this does not happen. Therefore $P_{p, \nu}(N)$ is positive for any $N$. In particular, it remains positive when we evaluate it on $\widehat{N} \leq N$. We have

$$
Q_{\Lambda, M_{\Lambda}, p, \nu}^{\mathrm{BPS}}=\frac{1}{\sqrt{P_{p, \nu}(N)}} \sum_{\substack{R, \tau \\ l(R) \leq l(p)}} q_{p, \nu}^{R, \tau} \frac{f_{p, \nu}^{\max }(N)}{f_{R}(N)} \mathcal{O}_{\Lambda, M_{\Lambda}, R, \tau}
$$

We now prove this vanishes when we lower $N$ to some $\widehat{N}<l(p)$. The properties of $P_{p, \nu}(N)$ mean there are no divisions by zero to concern us, and we focus on the coefficients $\frac{f_{p, \nu}^{\max }(N)}{f_{R}(N)}$.

By construction, $Q_{\Lambda, M_{\Lambda}, p, \nu}$ reduces to a commutator trace when we lower $N$ to $\widehat{N}<$ $l(p)$. Therefore it must contain at least one $\mathcal{O}_{\Lambda, M_{\Lambda}, R, \tau}$ with $l(R)=l(p)$, otherwise it would remain $S_{n}$ orthogonal to commutator traces if we lowered $N$ to $\widehat{N}=l(p)-1$. Therefore

$$
f_{p, \nu}^{\max }(N) \supset \prod_{i=0}^{l(p)-1}(N-i)
$$

where $\supset$ means $f_{p, \nu}^{\max }$ contains these as factors. By definition

$$
f_{R}(N) \supset \prod_{i=0}^{l(R)-1}(N-i)
$$


it follows that if $R \in Y_{p, \nu}$, the coefficient in front of $\mathcal{O}_{\Lambda, M_{\Lambda}, R, \tau}$ contains

$$
\frac{f_{p, \nu}^{\max }(N)}{f_{R}(N)} \supset \prod_{i=l(R)}^{l(p)-1}(N-i)
$$

This ensures that if $l(p)>\widehat{N}$, all terms in the expansion (6.44) vanish when we lower $N$ to $\widehat{N}$. If $l(R)>\widehat{N}, \mathcal{O}_{\Lambda, M_{\Lambda}, R, \tau}$ vanishes by definition, while if $l(R) \leq \widehat{N}$, the factors in (6.47) set it to zero.

Therefore $Q_{\Lambda, M_{\Lambda}, p, \nu}^{\mathrm{BPS}}$ vanishes identically when we lower $N$ to $\widehat{N}<l(p)$, and hence this is an SEP-compatible basis for weakly coupled BPS operators. This justifies the statement made in section 3.1 that applying $\mathcal{G}_{N}$ to an SEP-compatible basis of pre-BPS operators gives an SEP-compatible basis of BPS operators.

An alternative viewpoint is to look at the physical $\mathcal{F}$-weighted inner product. We have

$$
\left|Q_{\Lambda, M_{\Lambda}, p, \nu}^{\mathrm{BPS}}\right|_{\mathcal{F}}^{2}=\left\langle Q_{\Lambda, M_{\Lambda}, p, \nu} \mid Q_{\Lambda, M_{\Lambda}, p, \nu}\right\rangle_{\mathcal{F}}=\frac{f_{p, \nu}^{\max }(N) P_{p, \nu}^{(F)}(N)}{P_{p, \nu}(N)}
$$

where the new polynomial in the numerator is

$$
P_{p, \nu}^{(F)}(N)=\sum_{\substack{R, \tau \\ l(R) \leq l(p)}}\left(q_{p, \nu}^{R, \tau}\right)^{2} \frac{f_{p, \nu}^{\max }(N)}{f_{R}(N)}
$$

The overall factor of $f_{p, \nu}^{\max }(N)$ in (6.48) ensures that it vanishes when we lower $N$ to some $\widehat{N}<l(p)$. As a consistency check, we also prove that it is non-vanishing when $\widehat{N} \geq l(p)$.

This relies on noticing that the linear factors in $\frac{f_{p, \nu}^{\max }(N)}{f_{R}(N)}$ are all of the form $(N-i)$ for $-n \leq i \leq l(p)-1$. Therefore $\frac{f_{p, \nu}^{\max }(\widehat{N})}{f_{R}(\widehat{N})}>0$ when $\widehat{N} \geq l(p)$. It follows that $P_{p, \nu}^{(F)}(\widehat{N})>0$, and the result follows.

In the planar $(N \rightarrow \infty)$ limit, applying $\mathcal{G}_{N}$ reduces to division by $N^{n}$, and therefore after $S_{n}$-normalising we have

$$
\left.Q_{\Lambda, M_{\Lambda}, p, \nu}^{\mathrm{BPS}}\right|_{N \rightarrow \infty}=Q_{\Lambda, M_{\Lambda}, p, \nu}
$$

Since $Q_{\Lambda, M_{\Lambda}, p, \nu}$ is a symmetrised trace at large $N$, this means commutator traces are sub-leading in the large $N$ multi-trace expansion of $Q_{\Lambda, M_{\Lambda}, p, \nu}^{\mathrm{BPS}}$.

Having constructed an SEP-compatible basis of BPS operators, the natural next question to ask is whether we can find a formula for their correlators. This uses the physical $\mathcal{F}$-weighted inner product. From the hermiticity of $\mathrm{U}(2)$, the $Q_{\Lambda, M_{\Lambda}, p, \nu}^{\mathrm{BPS}}$ are $\mathcal{F}$-orthogonal in the $\Lambda, M_{\Lambda}$ labels, but in general are not in $p, \nu$. Therefore studying correlators involves calculating a matrix of inner products. In the next section we $\mathcal{F}$-orthogonalise the $Q_{\Lambda, M_{\Lambda}, p, \nu}^{\mathrm{BPS}}$ in order to produce an $\mathcal{F}$-orthogonal SEP-compatible basis in which it is easier to study properties of correlators.

In this section we have normalised operators using the $S_{n}$ inner product. This is in some sense un-natural, as the $Q_{\Lambda, M_{\Lambda}, p, \nu}^{\mathrm{BPS}}$ are not orthogonal in this inner product. There is another, alternative normalisation we could consider. In (6.44) we have a complicated 
normalisation factor of $\left(P_{p, \nu}(N)\right)^{-1 / 2}$, which if removed, would mean the coefficients of the free field operators (and multi-traces) would be expressible purely as polynomials in $N$. This is a natural normalisation to consider, and is given simply by

$$
f_{p, \nu}^{\max } \mathcal{G}_{N} Q_{\Lambda, M_{\Lambda}, p, \nu}
$$

However, this is more difficult than the $S_{n}$ normalisation, since it involves knowing which free field operators appear in the expansion of $Q_{\Lambda, M_{\Lambda}, p, \nu}$, and the free field operators are computationally expensive to construct. In contrast, the $S_{n}$ normalisation can be deduced purely from an expression in terms of multi-traces, which is obtainable explicitly from the construction of $Q_{\Lambda, M_{\Lambda}, p, \nu}$.

In section 6.9 we prove that for $\Lambda=[n]$, the $Q_{\Lambda, M_{\Lambda}, p, \nu}$ exactly reproduce the free field basis $\mathcal{O}_{\Lambda, M_{\Lambda}, R, \tau}$ up to a choice of multiplicity basis, and conjecture that the same happens for $\Lambda=[n-1,1]$. If this is true, then for these $\Lambda$, applying $\mathcal{G}$ and $S_{n}$ normalising leaves the operators unchanged and we have

$$
Q_{\Lambda, M_{\Lambda}, p, \nu}^{\mathrm{BPS}}=Q_{\Lambda, M_{\Lambda}, p, \nu}=\mathcal{O}_{\Lambda, M_{\Lambda}, R=p, \tau=\nu}
$$

\section{4 $\mathcal{F}$-orthogonalisation}

To define an orthogonal SEP-compatible basis, we Gram-Schmidt orthogonalise the $Q_{\Lambda, M_{\Lambda}, p, \nu}^{\mathrm{BPS}}$ operators using the physical $\mathcal{F}$-weighted inner product and the same ordering defined in (6.6). We denote the orthogonalised operators by $\bar{S}_{\Lambda, M_{\Lambda}, p, \nu}^{\mathrm{BPS}}$, and after normalising them to have $S_{n}$ norm $1, S_{\Lambda, M_{\Lambda}, p, \nu}^{\mathrm{BPS}}$.

Let $p_{\Lambda ; N}^{*}$ be the largest partition with $\mathcal{M}_{\Lambda, p_{\Lambda ; N}^{*}}>0$ and $l\left(p_{\Lambda ; N}^{*}\right) \leq N$. Then in analogy to $(6.7),(6.8)$ and $(6.9)$, we have

$$
\begin{aligned}
\bar{S}_{\Lambda, M_{\Lambda}, p_{\Lambda, N}^{*}}^{\mathrm{BPS}} & =Q_{\Lambda, M_{\Lambda}, p_{\Lambda ; N}^{*}}^{\mathrm{BPS}} \\
\bar{S}_{\Lambda, M_{\Lambda}, p, \nu}^{\mathrm{BPS}} & =Q_{\Lambda, M_{\Lambda}, p, \nu}^{\mathrm{BPS}}-\sum_{\substack{(q, \eta)>(p, \nu) \\
l(q) \leq N}} \frac{\left\langle Q_{\Lambda, M_{\Lambda}, p, \nu}^{\mathrm{BPS}}, \bar{S}_{\Lambda, M_{\Lambda}, q, \eta}^{\mathrm{BPS}}\right\rangle_{\mathcal{F}}}{\left\langle\bar{S}_{\Lambda, M_{\Lambda}, q, \eta}^{\mathrm{BPS}}, \bar{S}_{\Lambda, M_{\Lambda}, q, \eta}^{\mathrm{BPS}}\right\rangle_{\mathcal{F}}} \bar{S}_{\Lambda, M_{\Lambda}, q, \eta}^{\mathrm{BPS}} \\
S_{\Lambda, M_{\Lambda}, p, \nu}^{\mathrm{BPS}} & =\frac{\bar{S}_{\Lambda, M_{\Lambda}, p, \nu}^{\mathrm{BPS}}}{\sqrt{\left\langle\bar{S}_{\Lambda, M_{\Lambda}, p, \nu}^{\mathrm{BPS}} \mid \bar{S}_{\Lambda, M_{\Lambda}, p, \nu}^{\mathrm{BPS}}\right\rangle_{S_{n}}}}
\end{aligned}
$$

where $l(p) \leq N$.

Note the difference in the starting point of the orthogonalisation compared to (6.7). When $S_{n}$ orthogonalising the pre-BPS operators, we began with $p_{\Lambda}^{*}=p_{\Lambda ; \infty}^{*}$ even if $l\left(p_{\Lambda}^{*}\right)>$ $N$, whereas this time we apply the finite $N$ cut-off to the partitions being orthogonalised.

From the construction it follows that $Q_{\Lambda, M_{\Lambda}, q, \eta}^{\mathrm{BPS}}$ only contributes to $S_{\Lambda, M_{\Lambda}, p, \nu}^{\mathrm{BPS}}$ if $q \geq p$. Upon lowering $N$ to $\widehat{N}$, since the $Q^{\text {BPS }}$ operators with $l(q)>\widehat{N}$ vanish identically, the $S^{\mathrm{BPS}}$ will also vanish for $l(p)>\widehat{N}$, and therefore this is an SEP-compatible basis.

Note this relies on $Q_{\Lambda, M_{\Lambda}, q, \eta}^{\mathrm{BPS}}$ not appearing with a coefficient of $\frac{1}{N-i}$ for $i \leq l(q)$, as this would upset the SEP-compatibility in a way similar to that described in (6.29). As in the previous section, the $S_{n}$ normalisation ensures this does not occur. 
In the planar $(N \rightarrow \infty)$ limit, the physical $\mathcal{F}$-weighted inner product reduces to $N^{n}$ times the $S_{n}$ inner product, therefore $\mathcal{F}$-orthogonalising is equivalent to $S_{n}$-orthogonalising. From (6.50), the $Q_{\Lambda, M_{\Lambda}, p, \nu}^{\mathrm{BPS}}$ operators reduce to the pre-BPS operators $Q_{\Lambda, M_{\Lambda}, p, \nu}$ in the planar limit, which are already $S_{n}$ orthonormal. Therefore

$$
\left.S_{\Lambda, M_{\Lambda}, p, \nu}^{\mathrm{BPS}}\right|_{N \rightarrow \infty}=Q_{\Lambda, M_{\Lambda}, p, \nu}
$$

Below (6.9) we explained that only those free field operators with $l(R) \leq l(p)$ contribute to $Q_{\Lambda, M_{\Lambda}, p, \nu}$. After orthogonalisation, the $S_{\Lambda, M_{\Lambda}, p, \nu}^{\mathrm{BPS}}$ can admit operators with $l(R)>l(p)$, but (6.56) proves that these are sub-leading at large $N$.

Furthermore, just as discussed for $Q_{\Lambda, M_{\Lambda}, p, \nu}^{\mathrm{BPS}}$ below (6.50), commutator traces are subleading in the large $N$ multi-trace expansion of $S_{\Lambda, M_{\Lambda}, p, \nu}^{\mathrm{BPS}}$.

\subsubsection{The $\Lambda=[2,2]$ example}

We begin with the operators (6.31) and (6.32). After $\mathcal{F}$-orthogonalising and normalising with respect to the $S_{n}$ inner product, we obtain

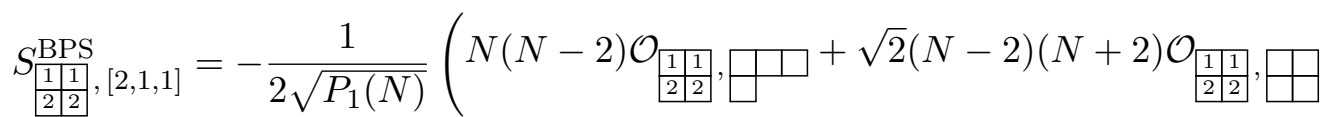

$$
\begin{aligned}
& \left.+3 N(N+2) \mathcal{O}_{\frac{11}{212},}, \boxminus\right)
\end{aligned}
$$

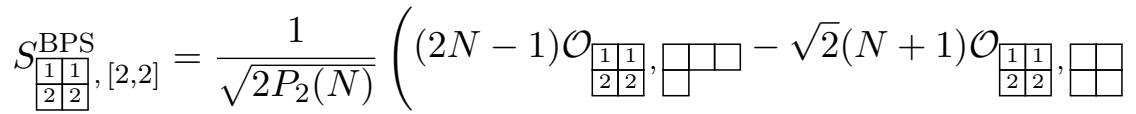

$$
\begin{aligned}
& \left.+\mathcal{O}_{\frac{1}{\frac{1}{2} 2}, \frac{1}{2},}, \square\right)
\end{aligned}
$$

where the normalisation polynomials $P_{1}$ and $P_{2}$ are given by

$$
\begin{aligned}
& P_{1}(N)=3 N^{4}+8 N^{3}+6 N^{2}+8 \\
& P_{2}(N)=3 N^{2}+2
\end{aligned}
$$

Written in terms of traces, these operators are

$$
\begin{gathered}
S_{\frac{111}{2 \frac{1}{2}},[2,1,1]}^{\mathrm{BPS}}=\frac{1}{2 \sqrt{3 P_{1}(N)}}\left[\left(N^{2}+2 N-2\right) t_{[2,1,1]}-2 N(N+1) t_{[2,2]}\right. \\
+4(N+1) \operatorname{Tr} X[X, Y] Y] \\
S_{\frac{211}{2 \frac{1}{2},},[2,2]}^{\mathrm{BPS}}=\frac{1}{\sqrt{6 P_{2}(N)}}\left[N\left(t_{[2,1,1]}+t_{[2,2]}\right)-2 \operatorname{Tr} X[X, Y] Y\right]
\end{gathered}
$$

\subsection{A shorter algorithm}

In the previous sections we have given a method to derive an orthogonal SEP-compatible basis $S_{\Lambda, M_{\Lambda}, p, \nu}^{\mathrm{BPS}}$. This method goes through two orthogonalisation procedures, applying $\mathcal{G}_{N}$ inbetween. The first step used the $S_{n}$ inner product and involved all the partitions, 
the second used the $\mathcal{F}$-weighted inner product and only included those partitions with $l(p) \leq N$. This means the partitions with $l(p) \leq N$ are orthogonalised among each other twice. We now prove that one may simplify the first orthogonalisation procedure to only orthogonalise against those $p$ with $l(p)>N$ and still obtain the same final output. This simplifies the computational requirements, and is also conceptually simpler, for reasons that will be outlined below.

To prove this streamlined procedure produces the same BPS operators, we first recall some useful facts.

\subsubsection{General properties of Gram-Schmidt orthogonalisation}

Consider two bases $\left\{v_{i}\right\}$ and $\left\{e_{i}\right\}$ of a vector space $V$, where the second is orthonormal. Then orthogonalising $v_{i}$ results in the basis $e_{i}$ (up to normalisation constants) if and only if the matrix connecting the two is lower diagonal.

$$
\left\{v_{i}\right\} \stackrel{\mathrm{GS}}{\longrightarrow}\left\{e_{i}\right\} \quad \Longleftrightarrow \quad e_{i}=\sum_{j \geq i} A_{i}^{j} v_{j}
$$

Note that the orthogonalisation process here is the opposite way round to the standard Gram-Schmidt orthogonalisation one would find in a textbook. Ordinarily, one starts with minimal $i$ and proceeds to larger $i$, and therefore obtains an upper diagonal $A$ matrix. In (6.63), we start with maximal $i$ and decrease, since this is the approach taken in (6.8).

Now introduce another basis $\left\{u_{i}\right\}$ for $V$, not necessarily orthogonal. This is related to $\left\{v_{i}\right\}$ by a matrix $B_{i}^{j}$

$$
u_{i}=\sum_{i} B_{i}^{j} v_{j}
$$

Then it follows from (6.63) that if $B_{i}^{j}$ is lower diagonal, $\left\{u_{i}\right\}$ and $\left\{v_{i}\right\}$ orthogonalise to the same orthonormal basis $\left\{e_{i}\right\}$ (up to normalisation constants).

\subsubsection{Back to SEP-compatible bases}

As explained below (6.3), the $S_{n}$ orthogonalisation of $\mathcal{M}_{N}^{\leq}$against $\mathcal{M}_{N}^{>}$gives us pre-BPS operators. In (6.8), the continued $S_{n}$ orthogonalisation among partitions with $l(p) \leq N$ gives us SEP-compatibility, but is not required for the operators to be pre-BPS.

To split these two steps us, define the operators $\bar{M}_{\Lambda, M_{\Lambda}, p, \nu}$ by

$$
\begin{aligned}
& \bar{M}_{\Lambda, M_{\Lambda}, p_{\Lambda}^{*}}=M_{\Lambda, M_{\Lambda}, p_{\Lambda}^{*}} \\
& \bar{M}_{\Lambda, M_{\Lambda}, p, \nu}=M_{\Lambda, M_{\Lambda}, p, \nu}-\sum_{\substack{(q, \eta)>(p, \nu) \\
l(q)>N}} \frac{\left\langle M_{\Lambda, M_{\Lambda}, p, \nu}, \bar{M}_{\Lambda, M_{\Lambda}, q, \eta}\right\rangle_{S_{n}}}{\left\langle\bar{M}_{\Lambda, M_{\Lambda}, q, \eta}, \bar{M}_{\Lambda, M_{\Lambda}, q, \eta}\right\rangle_{S_{n}}} \bar{M}_{\Lambda, M_{\Lambda}, q, \eta}
\end{aligned}
$$

where for $p$ with $l(p)>N$, we have the same caveats as mentioned around (6.8) regarding vanishing of operators. Namely, some of the $\bar{M}_{\Lambda, M_{\Lambda}, p, \nu}$ with $l(p)>N$ may vanish as the algorithm proceeds. These operators are excluded from the rest of the orthogonalisation; it is implicit that the sum in (6.66) does not run over these values of $q, \eta$. 
The operators $\bar{M}_{\Lambda, M_{\Lambda}, p, \nu}$ for $l(p) \leq N$ form a basis of pre-BPS operators (the space $\left.\overline{\mathcal{M}}_{N}^{\leq}\right)$, while the operators with $l(p)>N$ are merely a computational tool in order to find this basis, and serve no further purpose here. From now on, when we refer to the operators $\bar{M}_{\Lambda, M_{\Lambda}, p, \nu}$, we will implicitly mean only those with $l(p) \leq N$.

The equations (6.65) and (6.66) constitute an implementation of the orthogonalisation process described in (7.70).

The $\bar{M}_{\Lambda, M_{\Lambda}, p, \nu}$ operators were briefly mentioned above (6.4). The corresponding BPS operators, normalised in the $S_{n}$ inner product, are

$$
\bar{M}_{\Lambda, M_{\Lambda}, p, \nu}^{\mathrm{BPS}}=\frac{\mathcal{G}_{N} \bar{M}_{\Lambda, M_{\Lambda}, p, \nu}}{\left|\mathcal{G}_{N} \bar{M}_{\Lambda, M_{\Lambda}, p, \nu}\right|_{S_{n}}}
$$

Compare the construction (6.66) of the $\bar{M}_{\Lambda, M_{\Lambda}, p, \nu}$ operators with the construction (6.8) of the $S_{n}$ orthogonal basis $Q_{\Lambda, M_{\Lambda}, p, \nu}$. The algorithms are the same for those $p$ with $l(p)>N$, while for those $p$ with $l(p) \leq N$, the former stops the orthogonalisation at $q$ with $l(q)=N$ and the latter continues the orthogonalisation over all $q>p$. Consequently, performing a Gram-Schmidt orthogonalisation process on the $\bar{M}_{\Lambda, M_{\Lambda}, p, \nu}$ leads to the $Q_{\Lambda, M_{\Lambda}, p, \nu}$ operators. Stated more explicitly, if we replace the $M_{\Lambda, M_{\Lambda}, p, \nu}$ operators in (6.8) with $\bar{M}_{\Lambda, M_{\Lambda}, p, \nu}$ (and restrict $p$ and $q$ to have $l(p), l(q) \leq N)$, this would not change the output.

Since $Q_{\Lambda, M_{\Lambda}, p, \nu}$ can be obtained from $\bar{M}_{\Lambda, M_{\Lambda}, p, \nu}$ by Gram-Schmidt orthogonalisation, it follows from (6.63) that the two bases are related by a lower diagonal matrix

$$
Q_{\Lambda, M_{\Lambda}, p, \nu}=\sum_{\substack{(q, \eta) \geq(p, \nu) \\ l(q), l(p) \leq N}} a_{(p, \nu)}^{(q, \eta)} \bar{M}_{\Lambda, M_{\Lambda}, q, \eta}
$$

After applying $\mathcal{G}$ and normalising under the $S_{n}$ inner product, the equivalent BPS bases $Q_{\Lambda, M_{\Lambda}, p, \nu}^{\mathrm{BPS}}$ and $\bar{M}_{\Lambda, M_{\Lambda}, p, \nu}^{\mathrm{BPS}}$ are related by a rescaled lower diagonal matrix. Then from the discussion below (6.64), it follows that performing Gram-Schmidt orthogonalisation on either of the two bases in the physical $\mathcal{F}$-weighted inner product will result in the same final basis $S_{\Lambda, M_{\Lambda}, p, \nu}^{\mathrm{BPS}}$.

Therefore one may obtain the $S_{\Lambda, M_{\Lambda}, p, \nu}^{\mathrm{BPS}}$ basis in a simpler manner by $\mathcal{F}$-orthogonalising the $\bar{M}_{\Lambda, M_{\Lambda}, p, \nu}$ basis, much as we did in (6.53)-(6.55)

$$
\begin{aligned}
\bar{S}_{\Lambda, M_{\Lambda}, p_{\Lambda, N}^{*}}^{\mathrm{BPS}} & =\bar{M}_{\Lambda, M_{\Lambda}, p_{\Lambda, N}^{*}}^{\mathrm{BPS}} \\
\bar{S}_{\Lambda, M_{\Lambda}, p, \nu}^{\mathrm{BPS}} & =\bar{M}_{\Lambda, M_{\Lambda}, p, \nu}^{\mathrm{BPS}}-\sum_{\substack{(q, \eta)>(p, \nu) \\
l(q) \leq N}} \frac{\left\langle\bar{M}_{\Lambda, M_{\Lambda}, p, \nu}^{\mathrm{BPS}}, \bar{S}_{\Lambda, M_{\Lambda}, q, \eta}^{\mathrm{BPS}}\right\rangle_{\mathcal{F}}}{\left\langle\bar{S}_{\Lambda, M_{\Lambda}, q, \eta}^{\mathrm{BPS}}, \bar{S}_{\Lambda, M_{\Lambda}, q, \eta}^{\mathrm{BPS}}\right\rangle_{\mathcal{F}}} \bar{S}_{\Lambda, M_{\Lambda}, q, \eta}^{\mathrm{BPS}} \\
S_{\Lambda, M_{\Lambda}, p, \nu}^{\mathrm{BPS}} & =\frac{\bar{S}_{\Lambda, M_{\Lambda}, p, \nu}^{\mathrm{BPS}}}{\sqrt{\left\langle\bar{S}_{\Lambda, M_{\Lambda}, p, \nu}^{\mathrm{BPS}} \bar{S}_{\Lambda, M_{\Lambda}, p, \nu}^{\mathrm{BPS}}\right\rangle_{S_{n}}}}
\end{aligned}
$$

where $l(p) \leq N$.

This approach to producing the $S_{\Lambda, M_{\Lambda}, p, \nu}^{\mathrm{BPS}}$ operators still involves two orthogonalisation steps, but the first is now computationally less demanding, and can be completely skipped if 
$N \geq l\left(p_{\Lambda}^{*}\right)$. Moreover, there is a clearer conceptual separation between the two steps. The first one obtains pre-BPS operators, while the second one finds an SEP-compatible basis.

This process skips the $Q_{\Lambda, M_{\Lambda}, p, \nu}$ operators, but they still have physical relevance as the planar limit of $S_{\Lambda, M_{\Lambda}, p, \nu}^{\mathrm{BPS}}$, and were mathematically useful in proving the SEP-compatibility of these operators.

\subsection{Choice of SEP-compatible basis}

In the section 6.4.1 we derived an orthogonal SEP-compatible basis of operators for the $\Lambda=[2,2]$ sector. This sector was also investigated in appendix $\mathrm{C}$ of [32], and a different orthogonal SEP-compatible basis was found. One can check that the two bases span the same two dimensional space for any $N \geq 3$, and when $N=2$ we find exact agreement of operators.

The SEP-compatibility determines the behaviour of such a basis for $N=1,2$, but for higher $N$ there is a large degree of freedom. Define the $\mathcal{F}$-normalised operators $\widehat{S}_{\Lambda, M_{\Lambda}, p, \nu}^{\mathrm{BPS}}$ for a generic $\Lambda$ by

$$
\widehat{S}_{\Lambda, M_{\Lambda}, p, \nu}^{\mathrm{BPS}}=\frac{S_{\Lambda, M_{\Lambda}, p, \nu}^{\mathrm{BPS}}}{\left\langle S_{\Lambda, M_{\Lambda}, p, \nu}^{\mathrm{BPS}} \mid S_{\Lambda, M_{\Lambda}, p, \nu}^{\mathrm{BPS}}\right\rangle_{\mathcal{F}}}
$$

Let $c(N)$ and $s(N)$ be two functions of $N$ satisfying

$$
c(2)=1 \quad s(2)=0 \quad c(N)^{2}+s(N)^{2}=1
$$

We can use $c$ and $s$ to rotate the $\Lambda=[2,2] \mathcal{F}$-normalised basis operators to a new configuration

$$
\begin{aligned}
& \mathcal{O}_{1}=c(N) \widehat{S}_{\frac{\widehat{S}_{\frac{1}{2} 1}}{\mathrm{BPS}},[2,1,1]}+s(N) \widehat{S}_{\frac{1}{2 \frac{1}{2}}},[2,2] \\
& \mathcal{O}_{2}=-s(N) \widehat{S}_{\frac{1}{\frac{1}{2} \frac{1}{2}},[2,1,1]}^{\mathrm{BPS}}+c(N) \widehat{S}_{\frac{1}{\left.\frac{1}{2}\right|_{2}},[2,2]}
\end{aligned}
$$

To avoid problems with vanishing denominators at $N=2$, we normalise $\mathcal{O}_{1}, \mathcal{O}_{2}$ to have norm 1 in the $S_{n}$ inner product. These then define an alternative orthogonal, SEPcompatible basis for weak coupling quarter-BPS operators in the $\Lambda=[2,2]$ sector.

As $s(N)$ is determined by $c(N)$, there is effectively a function's worth of freedom in defining an orthogonal SEP-compatible basis. Clearly the vast majority of these will have definitions with far more complicated coefficients than those in (6.57) and (6.58) (or equivalently (6.61) and (6.62)). An interesting question is whether we can uniquely characterise a basis by having the 'nicest' coefficients. For example, the coefficients in the basis of [32] are of the form

$$
N+1 \pm \sqrt{2 N^{2}+1}
$$

These involve a sum of polynomial and surd terms, whereas the basis (6.57) and (6.58) has coefficients that are polynomial in $N$ up to an overall normalisation. One possible criterion would be to demand a basis with polynomial coefficients (up to overall normalisation) whose polynomials have minimal degree. If unique, these operators would in some sense 
be the 'simplest' orthogonal, SEP-compatible basis. It is reasonable to conjecture that $S_{\Lambda, M_{\Lambda}, p, \nu}^{\mathrm{BPS}}$ form this basis.

For more general $\Lambda$, take an $N$-dependent orthogonal rotation matrix $R(\Lambda ; N)$ of size $\mathcal{M}_{\Lambda} \times \mathcal{M}_{\Lambda}$, where

$$
\mathcal{M}_{\Lambda}=\sum_{\substack{p \vdash n \\ l(p) \leq N}} \mathcal{M}_{\Lambda, p}
$$

When evaluated at $\widehat{N} \leq N$, the matrix should split into diagonal blocks

$$
R(\Lambda ; \widehat{N})=\left(\begin{array}{cc}
R \leq(\Lambda ; \widehat{N}) & 0 \\
0 & R^{>}(\Lambda ; \widehat{N})
\end{array}\right)
$$

where $R^{>}(\Lambda ; \widehat{N})$ rotates those partitions with length $l(p)>\widehat{N}$ among themselves and $R \leq(\Lambda ; \widehat{N})$ rotates those partitions with length $l(p) \leq \widehat{N}$ among themselves.

Then consider the $S_{n}$ normalised versions of the operators

$$
\sum_{\substack{q, \eta \\ l(q) \leq N}} R(\Lambda ; N)_{q, \eta}^{p, \nu} \widehat{S}_{\Lambda, M_{\Lambda}, p, \nu}^{\mathrm{BPS}}
$$

These form an alternative orthogonal, SEP-compatible basis for the weakly coupled quarter-BPS sector.

\subsection{Physical norms of BPS operators}

For the free field operators (2.64), the physical norm $f_{R}$ is a polynomial in $N$ that is closely related to the corresponding Young diagram $R$. It has mathematical significance as the numerator of the Weyl dimension formula for $\mathrm{U}(N)$ representations. We now investigate the physical norms of the weak coupling BPS operators $S_{\Lambda, M_{\Lambda}, p, \nu}^{\mathrm{BPS}}$, beginning with an example at $\Lambda=[2,2]$. These operators are given in (6.57) and (6.58), and have physical $\mathcal{F}$-weighted norms

$$
\begin{aligned}
& \left|S_{\frac{1}{\frac{\mathrm{BPS}}{2} 2},[2,1,1]}^{\mathrm{B}}\right|^{2}=\frac{\left(3 N^{2}+4 N-2\right)(N+2)(N+1) N^{2}(N-1)(N-2)}{P_{1}} \\
& \left|S \frac{S_{\frac{11}{12}}^{\mathrm{BPS}},[2,2]}{2 \frac{2}{2}}\right|^{2}=\frac{\left(3 N^{2}+4 N-2\right)(N+1) N^{2}(N-1)}{P_{2}}
\end{aligned}
$$

This has two key features that will generalise. Firstly, the liner factors in the norms reflect the SEP-compatibility, enforcing that the first operator vanish for when we lower $N$ to $\widehat{N}=1,2$ and the second operator vanish when we lower $N$ to $\widehat{N}=1$. However, both norms have more linear factors than just those required by SEP-compatibility. For these two $p$, the numerators contain $f_{p}$ as a factor. This does not generalise to all $p$; in (B.175) and (B.179) we see that the numerators in the norms of $p=[2,2,2]$ and $[3,3]$ operators are one linear factor short of containing $f_{p}$. It is unclear whether these are exceptions, or whether at large $n$, very few operators contain $f_{p}$ in the numerator of the norm. It would be interesting to understand the linear factors that appear in the numerator and whether these have a physical interpretation. 
Secondly, the numerators share a factor of $\left(3 N^{2}+2 N-2\right)$. In appendices A, B and C we see that consecutive partitions (in the ordering (6.6)) share a complicated polynomial factor in the numerators. We believe this generalises to larger $n$, though it may be an artefact of the orthogonalisation process. This is discussed further in appendix B.4.

While the numerators of BPS norms have interesting properties, we have not found any structure in the denominators. They arise by dividing through by the square root of the $S_{n}$ norm, and from our numerical calculations do not seem to factorise into smaller units.

\subsubsection{Norms of operators with multiplicity}

The norms (6.80) and (6.81) can be considered as characteristic functions of $\Lambda$ along with the partitions $[2,1,1]$ and $[2,2]$ respectively, just as the norms of the free field basis are characteristic polynomials of the Young diagrams.

For the free field covariant basis (2.64), the physical norms depended only on $R$ and not the $\mathrm{U}(2)$ Young diagram $\Lambda$ or the multiplicity $\tau$. For the weak coupling basis, the norms can now depend on $\Lambda, p$ and $\nu$. The dependence on $\Lambda$ and $p$ is completely determined by the construction, while the dependence on $\nu$ is dictated by the choice of multiplicity space basis. We now outline a way of extracting functions of $N$ that do not depend on this choice, and are therefore associated to the pair $p, \Lambda$.

In (6.72), a rescaled BPS basis $\widehat{S}_{\Lambda, M_{\Lambda}, p, \nu}^{\mathrm{BPS}}$ was defined, orthonormal in the physical $\mathcal{F}$-weighted inner product. A different choice of multiplicity basis would result in an orthogonal rotation of these operators, and any trace over the multiplicity index is therefore independent of the this choice.

In particular, consider the matrix of $S_{n}$ inner products. For a trivial multiplicity space, this would be a $1 \times 1$ matrix containing the reciprocal of the norm $\left|S_{\Lambda, M_{\Lambda}, p, \nu}^{\mathrm{BPS}}\right|^{2}$. The appropriate generalisation to non-trivial multiplicity should therefore be to take the reciprocal of the trace, and we should also divide by the dimension of the multiplicity space. So the invariant function is

$$
f_{\Lambda, p}=\left(\frac{1}{\mathcal{M}_{\Lambda, p}} \sum_{\nu=1}^{\mathcal{M}_{\Lambda, p}}\left\langle\widehat{S}_{\Lambda, M_{\Lambda}, p, \nu}^{\mathrm{BPS}} \mid \widehat{S}_{\Lambda, M_{\Lambda}, p, \nu}^{\mathrm{BPS}}\right\rangle\right)^{-1}
$$

Note that the hermiticity properties of $\mathrm{U}(2)$ imply that we can choose any semi-standard tableau $M_{\Lambda}$ and it will not affect the calculation.

We can also use the square/cube/... of the $S_{n}$ inner product matrix to extract further basis-invariant functions of $N$. Let $A$ be the $S_{n}$ inner product matrix. Then we have

$$
f_{\Lambda, p}^{(k)}=\left(\frac{1}{\mathcal{M}_{\Lambda, p}} \operatorname{Tr} A^{k}\right)^{-\frac{1}{k}}
$$

Where we have taken the $k$ th root in order to have functions of the same degree in $N$. This stack of powers only goes up to $k \leq \mathcal{M}_{\Lambda, p}$ before the invariants are no longer independent.

In appendix $\mathrm{B}$, we see two examples of non-trivial multiplicity spaces in the $\Lambda=[4,2]$ sector, both of dimension two. In section B.3 we show the calculation for $p=[2,2,1,1]$ in some detail, while we are more schematic for $p=[3,2,1]$. 
In both examples, the numerator of $f_{\Lambda, p}^{(2)}$ is the same as $f_{\Lambda, p}^{(1)}$, though the denominator is not. As discussed below (6.81), this is further evidence that we should only look at the numerators of the BPS norms, giving a single characteristic function for a given $\Lambda, p$.

\subsection{Longest $p$ for a given $\Lambda$ and explicit quarter-BPS operators}

Due to the computational nature of the orthogonalisation process to derive SEP-compatible BPS operators, it is difficult to give explicit formulae for many of the $S_{\Lambda, M_{\Lambda}, p, \nu}^{\mathrm{BPS}}$ operators. The exception is for $p_{\Lambda}^{*}$, the longest partition with $\mathcal{M}_{\Lambda, p}>0$. Shortly, we will prove that this has $\mathcal{M}_{\Lambda, p_{\Lambda}^{*}}=1$, so we drop the multiplicity index. Provided $N \geq l\left(p_{\Lambda}^{*}\right)$, the operators with partition $p_{\Lambda}^{*}$ do not get orthogonalised, so we have the formula

$$
S_{\Lambda, M_{\Lambda}, p_{\Lambda}^{*}}^{\mathrm{BPS}}=\frac{\mathcal{G}_{N} M_{\Lambda, M_{\Lambda}, p_{\Lambda}^{*}}}{\left|\mathcal{G}_{N} M_{\Lambda, M_{\Lambda}, p_{\Lambda}^{*}}\right|_{S_{n}}}
$$

where

$$
\begin{aligned}
& M_{\Lambda, M_{\Lambda}, p_{\Lambda}^{*}}=\sum_{\mathbf{p} \vdash\left(n_{1}, n_{2}\right): p(\mathbf{p})=p_{\Lambda}^{*}} B_{\Lambda, M_{\Lambda}, p_{\Lambda}^{*}}^{\mathbf{p}} M_{\mathbf{p}} \\
& =\sum_{\substack{\mathbf{p} \vdash\left(n_{1}, n_{2}\right): p(\mathbf{p})=p_{\Lambda}^{*} \\
\mathbf{q} \vdash\left(n_{1}, n_{2}\right)}} B_{\Lambda, M_{\Lambda}, p_{\Lambda}^{*}}^{\mathbf{p}} \widetilde{C}_{\mathbf{p}}^{\mathbf{q}} T_{\mathbf{q}}
\end{aligned}
$$

and we have used (5.13) to express $M_{\Lambda, M_{\Lambda}, p_{\Lambda}^{*}}$ in terms of $M_{\mathbf{p}},(4.23)$ to write $M_{\mathbf{p}}$ in terms of $T_{\mathbf{q}}$, and $T_{\mathbf{q}}$ is the symmetrised trace operator (3.4).

Explicit formulae for other $S_{\Lambda, M_{\Lambda}, p, \nu}^{\mathrm{BPS}}$ operators in each $\Lambda$ sector are much more difficult to write down as they involve first orthogonalising down the partitions. Of course one may find non-orthogonal BPS operators by applying $\mathcal{G}_{N}$ to the covariant monomials $M_{\Lambda, M_{\Lambda}, p, \nu}$ (provided $N \geq l\left(p_{\Lambda}^{*}\right)$ ).

We can use the results of sections 5.5 and 5.6 to find $p_{\Lambda}^{*}$ explicitly. As discussed at the end of section 5.6, we consider a partition $p=\left[1^{\mu_{1}}\right]+\bar{p}$ with a single dominant column of length $\mu_{1}$ attached to a smaller partition $\bar{p} \vdash \bar{n}=n-\mu_{1}$. By rectangle rotations, this has the same multiplicities as a single dominant row partition with first row of length $\lambda_{1}=\mu_{1}-l(\bar{p})$ above a smaller partition $\hat{p}=\left[1^{l(\bar{p})}\right]+\bar{p}$, where $\hat{p} \vdash \hat{n}=l(\bar{p})+\bar{n}$ and we are using the notation of section 5.6. We give an example of these relations between single dominant column and single dominant row partitions in (5.96).

Applying the general formula (5.94) for a partition with a single dominant row, we see that $\Lambda=\left[\Lambda_{1}, \Lambda_{2}\right]$ only has non-trivial multiplicity with $p$ if

$$
\Lambda_{2} \leq n-\mu_{1}+l(\bar{p})
$$

Rearranging to constrain $\mu_{1}$ in terms of $\Lambda$ and $\bar{p}$

$$
\mu_{1} \leq \Lambda_{1}+l(\bar{p})
$$

Not only does (5.94) give this constraint on $\mu_{1}$, it also tells us that the multiplicity is 1 if the inequality is saturated. If it is not quite saturated, and instead $\mu_{1}=\Lambda_{1}+l(\bar{p})-1$, 
then the multiplicity is the number of corners in $p$ minus 1 . Since $p$ has a dominant first column, this is just the number of corners in $\bar{p}$.

Therefore the maximum possible $\mu_{1}$ is obtained when $l(\bar{p})$ is at its largest. This occurs when $\bar{p}=\left[1^{\bar{n}}\right]$ and $l(\bar{p})=\bar{n}=n-\mu_{1}$. Plugging this in, we have

$$
\mu_{1} \leq n-\frac{\Lambda_{2}}{2}
$$

Therefore the maximal $\mu_{1}$ is $n-\left\lceil\frac{\Lambda_{2}}{2}\right\rceil$, with associated $\bar{p}=\left[1^{\left\lceil\frac{\Lambda_{2}}{2}\right\rceil}\right]$. If $\Lambda_{2}$ is even then (6.88) is saturated and the multiplicity is 1 . If $\Lambda_{2}$ is odd, then the multiplicity is the number of corners in $\bar{p}$, which is also 1 . These multiplicities agree with the explicit calculation for two column partitions in (5.82).

Stated fully, for $\Lambda=\left[\Lambda_{1}, \Lambda_{2}\right] \vdash n$, the longest $p$ with non-trivial multiplicity is

$$
p_{\Lambda}^{*}=\left[n-\left\lceil\frac{\Lambda_{2}}{2}\right\rceil,\left\lceil\frac{\Lambda_{2}}{2}\right\rceil\right]^{c}=\left[2^{\left\lceil\frac{\Lambda_{2}}{2}\right\rceil}, 1^{n-2\left\lceil\frac{\Lambda_{2}}{2}\right\rceil}\right]
$$

and this multiplicity is 1 .

\subsection{Orthogonalisation at $\Lambda=[n]$ and $[n-1,1]$}

In section 6.2.1, we observed that Gram-Schmidt orthogonalising the $M_{\Lambda, M_{\Lambda}, p, \nu}$ with $\Lambda=[4]$ or $[3,1]$ in the $S_{n}$ inner product led to the free field operators $\mathcal{O}_{\Lambda, M_{\Lambda}, R, \tau}$. We now prove that this behaviour is general for $\Lambda=[n]$, and motivate a conjecture that this also happens for $\Lambda=[n-1,1]$.

For this subsection, when we use $\Lambda$, we will be referring specifically to $\Lambda=[n]$ or $[n-1,1]$, and stated results will apply only to those $\Lambda$.

Recall that for these $\Lambda$, a free field BPS operator is also a weak coupling pre-BPS operators. Therefore we have expansions of the form

$$
\mathcal{O}_{\Lambda, M_{\Lambda}, R, \tau}=\sum_{p, \nu} b_{R, \tau}^{p, \nu} M_{\Lambda, M_{\Lambda}, p, \nu} \quad M_{\Lambda, M_{\Lambda}, p, \nu}=\sum_{R, \tau}\left(b^{-1}\right)_{p, \nu}^{R, \tau} \mathcal{O}_{\Lambda, M_{\Lambda}, R, \tau}
$$

Next, recall from (2.74)-(2.76) that the free field operators are orthogonal in the $S_{n}$ inner product. Therefore from the property (6.63) of Gram-Schmidt orthogonalisation, the $M_{\Lambda, M_{\Lambda}, p, \nu}$ will orthogonalise to $\mathcal{O}_{\Lambda, M_{\Lambda}, R, \tau}$ if and only if $b_{R, \tau}^{p, \nu}$ is lower diagonal. That is, if the coefficients satisfy

$$
b_{R, \tau}^{p, \nu}=0 \quad \text { if } \quad(p, \nu)<(R, \tau)
$$

where the comparison between multiplicities makes sense since the size of the multiplicity space for a given $p=R$ is the same for the two bases. This is proved in (5.66).

More generally, since neither basis specifies a choice of multiplicity space basis, it is sufficient to prove that

$$
b_{R, \tau}^{p, \nu}=0 \quad \text { if } \quad p<R
$$

then after choosing the multiplicity space bases appropriately, (6.92) and the result will follow. 


\subsection{1 $\Lambda=[n]$}

In the $\Lambda=[n]$ sector at field content $(n, 0)$, the covariant monomials $M_{\Lambda, M_{\Lambda}, p, \nu}$ reduce to the monomial symmetric functions $M_{p}$ defined in (2.10), while the free field operators $\mathcal{O}_{\Lambda, M_{\Lambda}, R, \tau}$ reduce to the Schur operators $s_{R}$ defined in terms of monomials in (2.16). From these definitions, the two bases are related by the (rescaled) Kostka numbers

$$
s_{R}=\sum_{p \vdash n} \frac{K_{R p}}{\prod_{i} p_{i} !} M_{p}
$$

The Kostka numbers are the number of semi-standard Young tableaux of shape $R$ and evaluation $p$, where these terms are defined in section 2.2.2. To prove (6.93) in this case we need

$$
K_{R p}=0 \quad \text { for } \quad R>p
$$

Consider $R>p$ with column lengths $R^{c}=\left[\lambda_{1}, \lambda_{2}, \ldots\right]$ and $p^{c}=\left[\rho_{1}, \rho_{2}, \ldots\right]$. By definition there is some $l$ for which $\lambda_{i}=\rho_{i}$ for all $i<l$ and $\lambda_{l}>\rho_{l}$. Now take a semi-standard Young tableaux of shape $R$ and evaluation $p$. The entries in the first column must strictly increase, so the entry at the bottom of the first column is $\geq \lambda_{1}$. Since the evaluation is $p$, the available numbers to use are $1,2, \ldots, \rho_{1}=\lambda_{1}$, so we must fill this column with exactly the numbers 1 to $\lambda_{1}$. Similarly the second column must be filled with the numbers 1 to $\lambda_{2}$ and so on until we reach the $l$ th column. At this point, the entry at the bottom of the lth column must be $\geq \lambda_{l}$, while the maximum available number to use is $\rho_{l}<\lambda_{l}$. So the Young tableaux cannot have evaluation $p$, and therefore $K_{R p}=0$.

This proves (6.93) for $\Lambda=[n]$ and the highest weight state $M_{\Lambda}$. Applying the $\mathrm{U}(2)$ lowering operator $\mathcal{J}_{-}$, the same will happen for any $M_{\Lambda}$ within the $\Lambda=[n]$ sector, and this gives the result.

\subsection{2 $\Lambda=[n-1,1]$}

For $\Lambda=[n-1,1]$, there are two principal reasons we might expect $Q_{\Lambda, M_{\Lambda}, p, \nu}$ to agree with $\mathcal{O}_{\Lambda, M_{\Lambda}, R, \tau}$. Firstly, we know both bases are SEP-compatible. Since the space of operators that vanish when $N \rightarrow N-1$ is well-defined, it follows that for a fixed length $k=l(p)=l(R)$, the two bases must have the same span

$$
\operatorname{Span}\left\{S_{\Lambda, M_{\Lambda}, p, \nu}: l(p)=k\right\}=\operatorname{Span}\left\{\mathcal{O}_{\Lambda, M_{\Lambda}, R, \tau}: l(R)=k\right\}
$$

Secondly, from (6.92) the multiplicity for a given $p$ matches the multiplicity for $R=p$. Mathematically

$$
\begin{aligned}
\operatorname{Dim}\left(\operatorname { S p a n } \left\{S_{\Lambda, M_{\Lambda}, p, \nu}: 1 \leq\right.\right. & \left.\left.\nu \leq \mathcal{M}_{\Lambda, p}\right\}\right) \\
& =\operatorname{Dim}\left(\operatorname{Span}\left\{\mathcal{O}_{\Lambda, M_{\Lambda}, R, \tau}: 1 \leq \tau \leq C(R, R, \Lambda)\right\}\right)
\end{aligned}
$$

A rigorous proof that the $Q_{\Lambda, M_{\Lambda}, p, \nu}$ and $\mathcal{O}_{\Lambda, M_{\Lambda}, R, \tau}$ operators match is more difficult. Numerical calculations for this paper indicate it holds true up to at least $n=7$, and we leave the general case as a conjecture. 


\subsection{Alternative algorithm}

There is an alternative approach to capturing the finite $N$ behaviour of the pre-BPS sector starting from the free field operators. Following a similar process to that given in sections 6.1-6.5, one may use this to derive an orthogonal SEP-compatible basis of BPS operators. This alternative algorithm is outlined in figure 4. At first glance, there is no reason to expect agreement between this and the $S_{\Lambda, M_{\Lambda}, p, \nu}^{\mathrm{BPS}}$ basis defined in (6.69). However our numerical calculations show that they do agree up to $n=6$, and we conjecture that this is a general result.

Start by considering the free field basis $\mathcal{O}_{\Lambda, M_{\Lambda}, R, \tau}$. This has a symmetrised trace component and a commutator trace component. As discussed around (5.38), there is a $\mathrm{U}(2)$-covariant basis for commutator traces that we will denote by $c_{\Lambda, M_{\Lambda}, p, \xi}$, where $p \vdash n$ is a partition that describes the trace structure of the commutator trace and $\xi$ is a multiplicity index. For an example of these operators see (B.26)-(B.30), where we give the highest weight states in the $\Lambda=[4,2]$ covariant commutator trace basis.

While this alternative algorithm is useful, in particular providing an independent computational check of the main algorithm developed in sections $6.1-6.5$, it does not provide a constructive method to produce the multiplicities investigated in section 5 .

Then $\mathcal{O}_{\Lambda, M_{\Lambda}, R, \tau}$ can be written

$$
\mathcal{O}_{\Lambda, M_{\Lambda}, R, \tau}=\sum_{p, \nu} b_{R, \tau}^{p, \nu} M_{\Lambda, M_{\Lambda}, p, \nu}+\sum_{p, \xi} d_{R, \tau}^{p, \xi} c_{\Lambda, M_{\Lambda}, p, \xi}
$$

The coefficients $b_{R, \tau}^{p, \nu}$ are a generalisation of those seen in (6.91) to generic $\Lambda$.

The expansion coefficients in (6.98) are only defined uniquely at $N \geq n$. For $N<n$, finite $N$ relations make the choice non-unique. We will choose to use the large $N$ coefficient, even when working at $N<n$. These coefficients are valid for all $N$ and are independent of $N$.

After removing the commutator trace component, we are left with

$$
\mathcal{O}_{\Lambda, M_{\Lambda}, R, \tau}^{\mathrm{symm}}=\mathcal{O}_{\Lambda, M_{\Lambda}, R, \tau}-\sum_{p, \xi} d_{R, \tau}^{p, \xi} c_{\Lambda, M_{\Lambda}, p, \xi}=\sum_{p, \nu} b_{R, \tau}^{p, \nu} M_{\Lambda, M_{\Lambda}, p, \nu}
$$

which is a redundant spanning set for symmetrised traces. These operators were considered in [32], where they were referred to as $\mathcal{O}_{\Lambda, M_{\Lambda}, R, \tau}^{S}$.

Since $\mathcal{O}_{\Lambda, M_{\Lambda}, R, \tau}^{\mathrm{symm}}$ are symmetrised traces, they form pre-BPS operators for $N \geq n$. We also know from the construction that if $N<l(R)$, the operator $\mathcal{O}_{\Lambda, M_{\Lambda}, R, \tau}^{\text {symm }}$ reduces to a commutator trace. However, if $l(R) \leq N<n$, it is not necessarily true that $\mathcal{O}^{\text {symm }}$ is orthogonal to all commutator traces. This is the same situation as the monomial basis, discussed in section 3.2.

We may therefore use the same processes described in sections 6.1-6.5 in order to find an orthogonal SEP-compatible basis for BPS operators. In this section, we sill use the route given in sections 6.1-6.4 rather than the shorter one from section 6.5.

In particular, we produce a basis $\mathcal{O}_{\Lambda, M_{\Lambda}, R, \rho}^{\text {orth }}$ by following the $S_{n}$-orthogonalisation procedure (6.7)-(6.9). We then apply $\mathcal{G}_{N}, \mathcal{F}$-orthogonalise the resulting operators and $S_{n^{-}}$ normalise. The final basis is then denoted by $\mathcal{O}_{\Lambda, M_{\Lambda}, R, \rho}^{\mathrm{BPS}}$. This algorithm is outlined in figure 4 . 
As the operators $\mathcal{O}_{\Lambda, M_{\Lambda}, R, \tau}^{\mathrm{symm}}$ are linearly dependent, some of them will vanish during the orthogonalisation process. Unlike the orthogonalisation of monomials, this can occur for both $l(R)>N$ and $l(R) \leq N$. At such a point, remove that operator and continue with the orthogonalisation. This means the multiplicities for each pair $\Lambda, R$ with $l(R) \leq N$ could reduce, and in some cases will reduce to zero. Denote the reduced multiplicity for a pair by $\mathcal{M}_{\Lambda, R}^{\text {orth }}$. To indicate this reduction, we use a multiplicity index $\rho$ for the orthogonalised operators rather than $\tau$.

The $\mathcal{O}_{\Lambda, M_{\Lambda}, R, \rho}^{\text {orth }}$ with $l(R) \leq N$ form a $S_{n}$-orthogonal SEP-compatible basis for pre-BPS operators. The $\Lambda, M_{\Lambda}$ labels match the equivalents in $Q_{\Lambda, M_{\Lambda}, p, \nu}$, and by similar reasoning to (6.96), the length of $R$ must match the length of $p$. However, a priori, there is no reason to suspect that the $R$ label should match the $p$ label, or even that the multiplicities should be the same for any given partition.

From our numerical calculations up to $n=6$, we find that for each pair $\Lambda, R$ the multiplicities match, and the span of the operators with those labels is the same. Mathematically,

$$
\begin{aligned}
\mathcal{M}_{\Lambda, R}^{\text {orth }} & =\mathcal{M}_{\Lambda, p=R} \\
\operatorname{Span}\left\{\mathcal{O}_{\Lambda, M_{\Lambda}, R, \rho}^{\text {orth }}: 1 \leq \rho \leq \mathcal{M}_{\Lambda, R}^{\text {orth }}\right\} & =\operatorname{Span}\left\{Q_{\Lambda, M_{\Lambda}, p=R, \nu}: 1 \leq \nu \leq \mathcal{M}_{\Lambda, p}\right\}
\end{aligned}
$$

We conjecture that this is a general result for all $\Lambda, R$.

From this, it clearly follows that the BPS bases $\mathcal{O}_{\Lambda, M_{\Lambda}, R, \rho}^{\mathrm{BPS}}$ and $S_{\Lambda, M_{\Lambda}, p, \nu}^{\mathrm{BPS}}$ also match.

We can consider (6.100) and (6.101) as a generalisation to all $\Lambda$ of the orthogonalisation results discussed in section 6.9 for $\Lambda=[n]$ and $[n-1,1]$. In that case, we showed that to prove the results, it was sufficient for the coefficients in (6.91) to be lower diagonal in partition indices. We now prove a proposition that generalises this lower diagonality to arbitrary $\Lambda$.

The proposition involves the coefficients $b_{R, \tau}^{p, \nu}$ in (6.99). Consider the sub-matrix of $b_{R, \tau}^{p, \nu}$ with $p, R \geq q$ for some partition $q$ and denote this by $\left(b_{q}\right)_{R, \tau}^{p, \nu}$. Then $b_{q}$ is an $m_{q ; 1} \times m_{q ; 2}$ matrix where

$$
m_{q ; 1}=\sum_{p \geq q} \mathcal{M}_{\Lambda, p} \quad m_{q ; 2}=\sum_{R \geq q} C(R, R, \Lambda)
$$

Denote the rank of $b_{q}$ by $r_{q}$. This satisfies $r_{q} \leq m_{q ; 1}$.

Proposition. Suppose the coefficients $b_{R, \tau}^{p, \nu}$ are lower diagonal in the partition indices, so that

$$
\mathcal{O}_{\Lambda, M_{\Lambda}, R, \tau}^{\mathrm{symm}}=\sum_{\substack{p, \nu \\ p \geq R}} b_{R, \tau}^{p, \nu} M_{\Lambda, M_{\Lambda}, p, \nu}
$$

In addition, suppose that $b_{q}$ has maximal rank $r_{q}=m_{q ; 1}$ for each $q$. Then (6.100) and (6.101) hold.

Proof. Define

$$
\begin{array}{r}
V_{R}=\operatorname{Span}\left\{\mathcal{O}_{\Lambda, M_{\Lambda}, S, \tau}^{\text {symm }}: S \geq R, 1 \leq \tau \leq C(S, S, \Lambda)\right\} \\
\widetilde{V}_{R}=\operatorname{Span}\left\{M_{\Lambda, M_{\Lambda}, p, \nu}: p \geq R, 1 \leq \nu \leq \mathcal{M}_{\Lambda, p}\right\}
\end{array}
$$




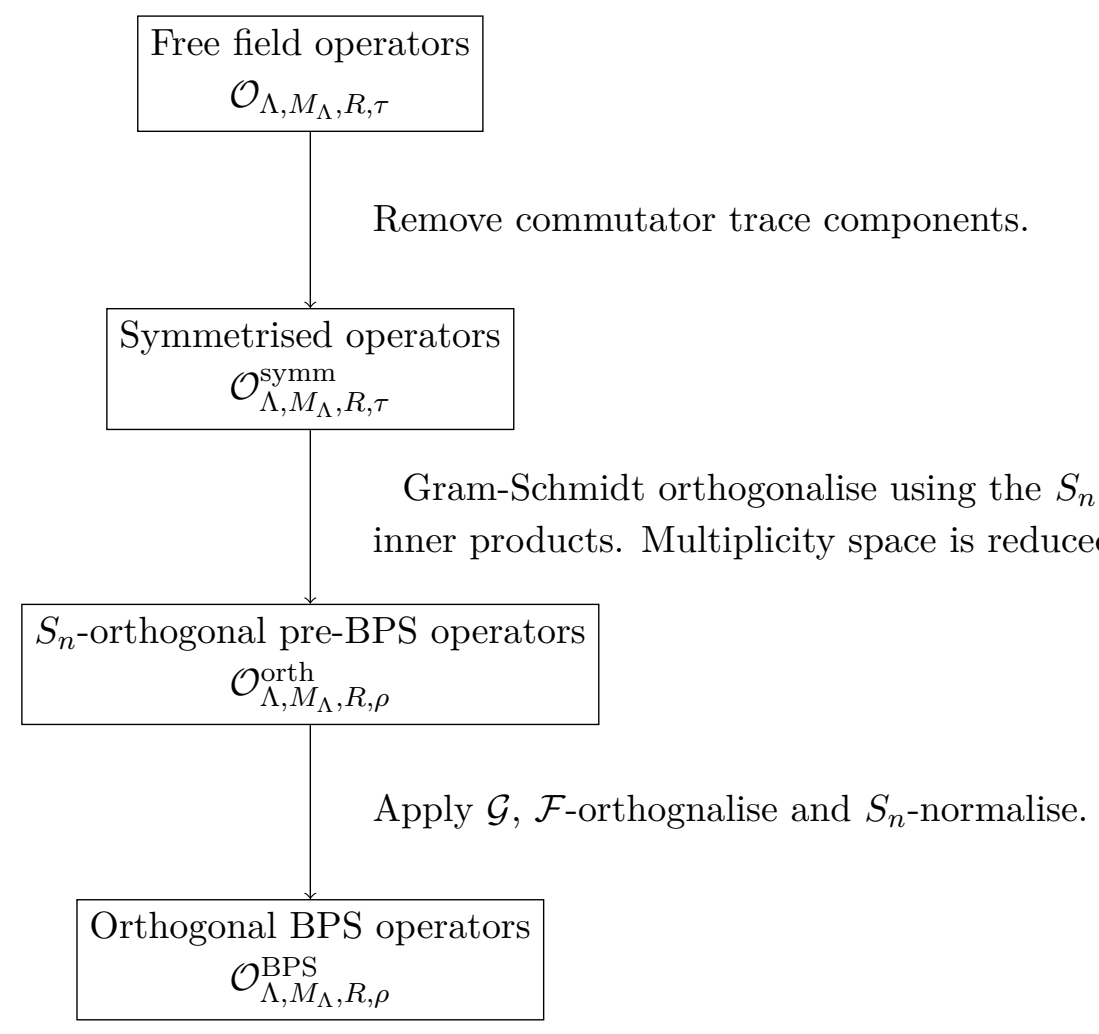

Figure 4. Outline of the alternative algorithm of this section. Our numerical calculations suggest that $\mathcal{O}_{\Lambda, M_{\Lambda}, R, \rho}^{\mathrm{BPS}}$ agrees (up to a choice of multiplicity basis) with the operators $S_{\Lambda, M_{\Lambda}, p, \nu}^{\mathrm{BPS}}$ derived from the algorithm in figure 2 .

It follows from (6.103) and the maximal rank condition for $b_{R}$ that $V_{R}=\widetilde{V}_{R}$.

Denote the partition immediately higher than $R$ by $R+1$. By construction, for $R$ with $l(R) \leq N$, the additional monomials included in $\widetilde{V}_{R}$ by lowering $R+1$ to $R$ are linearly independent, and therefore the dimension increases by $\mathcal{M}_{\Lambda, p=R}$.

For $V_{R}$, we have $C(R, R, \Lambda)$ new operators included by lowering $R+1$ to $R$, but the dimension only increases by $\mathcal{M}_{\Lambda, R}^{\text {orth }}$. Then since $V_{R}=\widetilde{V}_{R}$, we have

$$
\mathcal{M}_{\Lambda, R}^{\text {orth }}=\mathcal{M}_{\Lambda, p=R}
$$

Therefore for $R$ with $l(R) \leq N$ we may choose the multiplicity space basis such that $\mathcal{O}_{\Lambda, M_{\Lambda}, R, \tau}^{\text {symm }}$ is linearly independent of $V_{R+1}$ if $1 \leq \tau \leq \mathcal{M}_{\Lambda, p=R}$, and is linearly dependent on $V_{R+1}$ if $\tau>\mathcal{M}_{\Lambda, p=R}$. Under the orthogonalisation procedure, those operators with $\tau>\mathcal{M}_{\Lambda, p=R}$ will vanish. We can therefore equivalently start the procedure with a reduced set of operators $\mathcal{O}_{\Lambda, M_{\Lambda}, R, \rho}^{\text {symm }}$ where we only consider $1 \leq \rho \leq \mathcal{M}_{\Lambda, p=R}$. The coefficients relating these with $M_{\Lambda, M_{\Lambda}, p, \nu}$ are still lower diagonal in partition indices.

Now split the orthogonalisation into two steps, first orthogonalising against the $p$ or $R$ with $l(p), l(R)>N$ using the $S_{n}$ inner product. For the monomials, this results in the $\bar{M} \Lambda, M_{\Lambda}, p, \nu$ operators given in (6.66). Let the equivalent operators for the $\mathcal{O}^{\text {symm }}$ orthogonalisation be $\bar{O}_{\Lambda, M_{\Lambda}, R, \rho}^{\mathrm{symm}}$. These are both basis for the set of pre-BPS operators, and 
are still related by coefficients that are lower diagonal in partition indices. Therefore, as discussed below (6.64), they must orthogonalise to the same basis, in particular

$$
\mathcal{O}_{\Lambda, M_{\Lambda}, R, \rho}^{\text {orth }}=Q_{\Lambda, M_{\Lambda}, p=R, \nu=\rho}
$$

We have proved that (6.103), along with the maximal rank condition, is sufficient for (6.100) and (6.101). By a similar argument, it is also a necessary condition, though we will not prove this here.

The maximal rank condition $r_{q}=m_{q ; 1}$ is equivalent to saying that we can choose bases for the free field and covariant monomial multiplicity spaces such that

$$
\begin{array}{ll}
b_{R=p, \tau}^{p, \nu}=0 & \text { if } \tau>\nu \\
b_{R=p, \tau}^{p, \nu} \neq 0 & \text { if } \tau=\nu
\end{array}
$$

where $1 \leq \nu \leq \mathcal{M}_{\Lambda, p}$ and $1 \leq \tau \leq C(p, p, \Lambda)$. Intuitively, this says that $b$ is lower diagonal with non-zero elements on the diagonal.

The coefficients $b_{R, \tau}^{p, \nu}$ are in some sense a covariant generalisation of the Kostka numbers, which have a nice combinatoric interpretation. It would be interesting to investigate whether there is a choice of normalisation for $\mathcal{O}_{\Lambda, M_{\Lambda}, p, \tau}^{\text {symm }}$ and $M_{\Lambda, M_{\Lambda}, p, \nu}$ such that these coefficients are integers, and whether they have any combinatoric interpretation.

\section{Vector space geometry in $\mathbb{C}\left(S_{n}\right)$ : BPS states from projectors for the intersection of finite $N$ and symmetrisation constraints in symmetric group algebras}

The construction algorithm for quarter BPS states in section 6 involves a $U(2)$ global symmetry which provides labels for the states constructed. Alongside the $\mathrm{U}(2)$ state labels, there is a $\mathrm{U}(N)$ Young diagram $Y(p)$ which emerges from the combinatorics of multisymmetric functions and their relation to the space of gauge invariant 2-matrix operators modulo commutators $[X, Y]$. We have observed in section 4.5 that the combinatorics of multi-symmetric functions admits a generalization to the multi-matrix case where we have $M$ different matrices $X^{1}, X^{2}, \cdots, X^{M}$. In this section we take a different viewpoint on the $M$-matrix system using permutations in analogy to the 1 and 2-matrix constructions in section 2.

This is used to investigate the vector space geometry in $\mathbb{C}\left(S_{n}\right)$ that lies behind the constructions of BPS bases in the previous sections, involving the interplay between a projector $\mathcal{P}_{H}$ for the $\mathrm{U}(M)$ flavour symmetry, a projector for the symmetrisation of traces $\mathcal{P}$ and an operator $\mathcal{F}_{N}$ whose kernel implements finite $N$ constraints. Restricting to the image of $\mathcal{F}_{N}$, there is a well-defined inverse $\mathcal{G}_{N}$. These operators are $M$-matrix analogues of $\mathcal{F}_{N}$ and $\mathcal{G}_{N}$ discussed in section 2. It was proved in [32] that BPS states are in

$$
\operatorname{Im}\left(\mathcal{G}_{N} \mathcal{P}_{N}\right)
$$


where $\mathcal{P}_{N}$ is an orthogonal (with respect to the $S_{n}$ inner product) projector acting on $\mathbb{C}\left(S_{n}\right)$ with

$$
\operatorname{Im} \mathcal{P}_{N} \equiv \operatorname{Im} \mathcal{P} \cap \operatorname{Im} \mathcal{F}_{N}
$$

The isomorphism between multi-symmetric functions and the ring of gauge invariants modulo commutators and the associated combinatorics of set partitions explained in section 4 allows us to give a general explicit construction of $\mathcal{P}_{N}$. This general discussion also serves to explain why the construction algorithm in section 6 is able to handle the finite $N$ constraints on BPS operators systematically. The flavour projection $\mathcal{P}_{H}$, for any chosen flavour group $H$, commutes with $\mathcal{P}, \mathcal{F}_{N}$ and can be done at the end.

\section{1 n-matrix model from permutations}

Let $V$ be the carrier space for the $N$-dimensional fundamental representation of $\mathrm{U}(N)$, and consider the $n$-fold tensor product $V^{\otimes n}$. $S_{n}$ acts on this space by permutation of the factors. Let

$$
\mathbb{Z}=Z_{1} \otimes Z_{2} \otimes \cdots \otimes Z_{n}
$$

where the $Z_{a}, a=1, \ldots, n$ are $n$ different $\mathrm{U}(N)$ matrices. Conjugating $\mathbb{Z}$ by $\sigma$ leads to

$$
\begin{aligned}
\sigma \mathbb{Z} \sigma^{-1} & =\sigma\left(Z_{1} \otimes Z_{2} \otimes \cdots \otimes Z_{n}\right) \sigma^{-1} \\
& =Z_{\sigma(1)} \otimes Z_{\sigma(2)} \otimes \cdots \otimes Z_{\sigma(n)} \\
& =\sigma(\mathbb{Z})
\end{aligned}
$$

where the last line defines $\sigma(\mathbb{Z})$.

Taking the trace over $V^{\otimes n}$ of $\sigma \mathbb{Z}$ gives a multi-trace featuring each of the $n$ matrices exactly once. A single cycle $\left(a_{1}, a_{2}, \ldots, a_{k}\right)$ in $\sigma$ leads to the single trace $\operatorname{Tr} Z_{a_{1}} Z_{a_{2}} \ldots Z_{a_{k}}$, while a permutation with several cycles leads to a multi-trace. Conversely, given a multitrace of $Z_{1}, Z_{2}, \ldots, Z_{n}$ in which each matrix only appears once, we can identify the permutation $\sigma \in S_{n}$ which produces this trace. Therefore we have an invertible map from $\mathbb{C}\left(S_{n}\right)$ into the space of $n$-matrix multi-traces of degree $(1,1, \ldots, 1)$

$$
\sigma \leftrightarrow \operatorname{Tr}(\sigma \mathbb{Z})
$$

Provided $N \geq n$, this is a vector space isomorphism between $\mathbb{C}\left(S_{n}\right)$ and the degree $(1,1, \ldots, 1)$ subspace of the ring of $\mathrm{U}(N)$ invariants of an $n$-matrix system.

There is a natural inner product on $\mathbb{C}\left(S_{n}\right)$. On permutations $\sigma, \tau \in S_{n}$ it is defined by

$$
g_{n}(\sigma, \tau)=\delta\left(\sigma \tau^{-1}\right)
$$

where $\delta$ is 1 on the identity and 0 on all other permutations. The inner product extends linearly to the rest of $\mathbb{C}\left(S_{n}\right)$. Using the isomorphism to $n$-matrix traces, this is the $n$ matrix equivalent of the $S_{n}$ inner product (at large $N$ ) defined for 1 and 2-matrix systems in (2.26) and (2.70).

We now look at three specialisations of this isomorphism. Firstly, to include the finite $N$ relations among traces. Secondly, to the degree $\left(n_{1}, n_{2}, \ldots, n_{M}\right)$ subspace of an $M$ matrix system. Thirdly, to symmetrised traces of the $n$-matrices. 


\subsubsection{Finite $N$ relations}

If $N<n$, there are finite $N$ relations among the traces to consider. These can be captured using the Fourier basis for $\mathbb{C}\left(S_{n}\right)$

$$
Q_{I J}^{R}=\frac{d_{R}}{n !} \sum_{\sigma \in S_{n}} D_{I J}^{R}(\sigma) \sigma
$$

where $R \vdash n$ labels a representation of $S_{n}$ and $I, J$ are basis indices within $R$. The finite $N$ cut-off on traces corresponds to removing those $Q_{I J}^{R}$ with $l(R)>N$.

We impose the finite $N$ cut-off using an operator $\mathcal{F}_{N}$

$$
\mathcal{F}_{N} \sigma=\Omega_{N} \sigma
$$

where $\Omega_{N}$ is an $N$ dependent element in the centre of $\mathbb{C}\left(S_{n}\right)$ defined in (2.22). On the Fourier basis it acts as

$$
\mathcal{F}_{N} Q_{I J}^{R}=\Omega_{N} Q_{I J}^{R}=f_{R}(N) Q_{I J}^{R}
$$

where the polynomial $f_{R}(N)$ is given in (2.29). Since $f_{R}=0$ when $l(R)>N$, this imposes the finite $N$ cut-off on the permutation space

$$
\begin{aligned}
\operatorname{Ker} \mathcal{F}_{N} & =\operatorname{Span}\left\{Q_{I J}^{R}: l(R)>N\right\} \\
\operatorname{Im} \mathcal{F}_{N} & =\operatorname{Span}\left\{Q_{I J}^{R}: l(R) \leq N\right\}
\end{aligned}
$$

For $\alpha \in \operatorname{Im} \mathcal{F}_{N}$, the inverse operator to $\mathcal{F}_{N}$ is

$$
\mathcal{G}_{N} \alpha=\Omega_{N}^{-1} \alpha
$$

where $\Omega_{N}^{-1}$ is defined in (2.33). On the Fourier basis it acts as

$$
\mathcal{G}_{N} Q_{I J}^{R}=\Omega_{N}^{-1} Q_{I J}^{R}= \begin{cases}\frac{1}{f_{R}} Q_{I J}^{R} & l(R) \leq N \\ 0 & l(R)>N\end{cases}
$$

Using standard character orthogonality relations, the Fourier basis for $\mathbb{C}\left(S_{n}\right)$ is orthogonal under the $g_{n}$ inner product

$$
g_{n}\left(Q_{I J}^{R}, Q_{K L}^{S}\right)=\frac{d_{R}}{n !} \delta^{R S} \delta_{I K} \delta_{J L}
$$

therefore $\operatorname{Im} \mathcal{F}_{N}$ and $\operatorname{Ker} \mathcal{F}_{N}$ are orthogonal subspaces of $\mathbb{C}\left(S_{n}\right)$

$$
\left(\operatorname{Im} \mathcal{F}_{N}\right)^{\perp}=\operatorname{Ker} \mathcal{F}_{N}
$$

where $(.)^{\perp}$ denotes the orthogonal complement of a vector space.

For $N<n$, define a second inner product $g_{n, N}$ on $\mathbb{C}\left(S_{n}\right)$ by

$$
g_{n, N}(\sigma, \tau)=\delta_{N}\left(\sigma \tau^{-1}\right)
$$


where $\delta_{N}$ is given in (2.27). Taken across to operators using the map (7.5), this is the $S_{n}$ inner product for $N<n$. It vanishes on $\operatorname{Ker} \mathcal{F}_{N}$, and is identical to $g_{n}$ on $\operatorname{Im} \mathcal{F}_{N}$. $\operatorname{In}$ particular, for an element $\alpha \in \operatorname{Im} \mathcal{F}_{N}$, we have

$$
g_{n, N}(\alpha, \tau)=g_{n}(\alpha, \tau)
$$

for any $\tau \in S_{n}$.

There are two ways of consider the map (7.5) as an isomorphism in the finite $N$ setting. Firstly, we could consider the quotient space. For $\alpha \in \operatorname{Ker} \mathcal{F}_{N}$, the corresponding trace $\operatorname{Tr} \alpha \mathbb{X}$ vanishes, and therefore any two elements of $\mathbb{C}\left(S_{n}\right)$ related by Ker $\mathcal{F}_{N}$ produce the same multi-trace. Therefore this is a map from the quotient space

$$
\mathbb{C}\left(S_{n}\right) / \operatorname{Ker} \mathcal{F}_{N}
$$

into the space of multi-traces of $n N \times N$ matrices of degree $(1,1, \ldots, 1)$, where now we can allow $N<n$.

Alternatively, we can take a representative of each equivalence class in the quotient space. The most natural way to do this is to take the element orthogonal to $\operatorname{Ker} \mathcal{F}_{N}$, which by (7.15) is in $\operatorname{Im} \mathcal{F}_{N}$. Therefore (7.5) is a vector space isomorphism between $\operatorname{Im} \mathcal{F}_{N}$ and the appropriate space of multi-traces.

\subsubsection{Flavour projection}

To study an $M$-matrix system $X_{1}, X_{2}, \ldots, X_{M}$, we set each of the $Z_{i}$ to be equal to one of the $X_{j}$. To study the degree $\left(n_{1}, n_{2}, \ldots, n_{M}\right)$ subspace of the matrix system, where $\sum_{i} n_{i}=n$, we set $Z_{1}, Z_{2}, \ldots, Z_{n_{1}}$ equal to $X_{1} ; Z_{n_{1}+1}, \ldots, Z_{n_{1}+n_{2}}$ equal to $X_{2}$ and so on. Then $\mathbb{Z}$ becomes

$$
\mathbb{X}=X_{1}^{\otimes n_{1}} \otimes X_{2}^{\otimes n_{2}} \otimes \cdots \otimes X_{M}^{\otimes n_{M}}
$$

Tracing $\sigma \mathbb{X}$ over $V^{\otimes n}$ leads to multi-traces of degree $\left(n_{1}, n_{2}, \ldots, n_{M}\right)$.

Let $H=S_{n_{1}} \times S_{n_{2}} \times \cdots \times S_{n_{M}}$. From (7.4) we see that for any $\tau \in H$ we have $\tau(\mathbb{X})=\mathbb{X}$. Therefore

$$
\operatorname{Tr}(\sigma \mathbb{X})=\operatorname{Tr}\left(\tau \sigma \tau^{-1} \mathbb{X}\right)
$$

Define a projector

$$
\mathcal{P}_{H}(\sigma)=\frac{1}{|H|} \sum_{\tau \in H} \tau \sigma \tau^{-1}
$$

To justify calling $\mathcal{P}_{H}$ a projector, we prove that it has the two properties

$$
\left(\mathcal{P}_{H}\right)^{2}=\mathcal{P}_{H} \quad\left(\mathcal{P}_{H}\right)^{\dagger}=\mathcal{P}_{H}
$$


where the Hermitian conjugate is with respect to the $g_{n}$ inner product. These are both simple consequences of the definition. We have

$$
\begin{aligned}
\left(\mathcal{P}_{H}\right)^{2}(\alpha) & =\mathcal{P}_{H}\left(\mathcal{P}_{H}(\alpha)\right) \\
& =\frac{1}{|H|^{2}} \sum_{\sigma, \tau \in H} \sigma \tau \alpha \tau^{-1} \sigma^{-1} \\
& =\frac{1}{|H|^{2}} \sum_{\sigma, \tau \in H} \sigma \alpha \sigma^{-1} \\
& =\frac{1}{|H|} \sum_{\mu \in H} \sigma \alpha \sigma^{-1} \\
& =\mathcal{P}_{H}(\alpha)
\end{aligned}
$$

and

$$
\begin{aligned}
g\left(\alpha, \mathcal{P}_{H}(\beta)\right) & =\frac{1}{|H|} \sum_{\sigma \in H} \delta\left(\alpha \sigma \beta^{-1} \sigma^{-1}\right) \\
& =\frac{1}{|H|} \sum_{\sigma \in H} \delta\left(\sigma^{-1} \alpha \sigma \beta^{-1}\right) \\
& =\frac{1}{|H|} \sum_{\sigma \in H} \delta\left(\sigma \alpha \sigma^{-1} \beta^{-1}\right) \\
& =g\left(\mathcal{P}_{H}(\alpha), \beta\right)
\end{aligned}
$$

The image of $\mathcal{P}_{H}$ is the sub-algebra of $\mathbb{C}\left(S_{n}\right)$ invariant under conjugation by $\mathcal{H}$, which we denote by $\mathcal{A}_{H}$. It follows from (7.20) that

$$
\operatorname{Tr}(\sigma \mathbb{X})=\operatorname{Tr}\left(\mathcal{P}_{H}(\sigma) \mathbb{X}\right)
$$

Therefore $\mathcal{A}_{H}$ is the algebra of permutations responsible for constructing multi-traces of degree $\left(n_{1}, \ldots, n_{M}\right)$. We call $\mathcal{P}_{H}$ the flavour projector, as it projects from the $n$-matrix system to one with $M$ flavours.

As discussed for the finite $N$ relations around (7.18), the map (7.5) gives an isomorphism between $\mathcal{A}_{H}$ or $\mathbb{C}\left(S_{n}\right) / \operatorname{Ker} \mathcal{P}_{H}$ and the space of $M$-matrix multi-traces of degree $\left(n_{1}, \ldots, n_{M}\right)$.

In section 2 we discussed the half-BPS sector, corresponding to $M=1$, and the free field quarter-BPS sector, corresponding to $M=2$. In the latter case we have $H=S_{n_{1}} \times S_{n_{2}}$, and the matching permutation centraliser algebra $\mathcal{A}_{H}$ was studied in [65]. The free-field eighth BPS sector is larger than just multi-traces of three matrices, since it also includes fermion contractions. However, the sub-sector consisting only of the scalar fields can be found by setting $M=3$. This subsector is dual to the class of eighth-BPS giant gravitons considered in [15].

\subsubsection{Symmetrised traces}

A symmetrised trace of $Z_{1}, Z_{2}, \ldots, Z_{n}$ is defined in a completely analogous manner to the 2 -matrix version in (3.3), allowing $a_{i} \in\{1,2, \ldots, n\}$ instead of $\{1,2\}$. Degree $(1, \ldots, 1)$ 
symmetrised traces are labelled by set partitions $\pi \in \Pi(n)$. These naturally correspond to $n$-vector partitions of weight $(1, \ldots, 1)$.

Take $b \subseteq\{1,2, \ldots, n\}$. Then there is an associated symmetrised single trace

$$
T_{b}=\operatorname{Str}\left(\prod_{i \in b} Z_{i}\right)
$$

where the symmetrisation implicit in Str means the ordering of the product is irrelevant. For a set partition $\pi \in \Pi(n)$ we have

$$
T_{\pi}=\prod_{b \in \pi} T_{b}
$$

where $b$ runs over the blocks of $\pi$.

The equivalent permutation picture comes from the set of permutations $\operatorname{Perms}(\pi)$, defined in (4.56). We have

$$
T_{\pi}=\frac{1}{|\operatorname{Perms}(\pi)|} \sum_{\sigma \in \operatorname{Perms}(\pi)} \sigma
$$

where we use the same notation $T_{\pi}$ for both the sum over permutations and the associated symmetrised trace operator. For the remainder of the section we only work with the permutation sum, so this ambiguity will not be an issue.

More generally, one can define a symmetrisation projector $\mathcal{P}$ which projects a permutation onto the space isomorphic to symmetrised traces. This is

$$
\mathcal{P}(\sigma)=\frac{1}{|G(\pi(\sigma))|} \sum_{\tau \in G(\pi(\sigma))} \tau \sigma \tau^{-1}
$$

where the set partition $\pi(\sigma)$ is defined naturally from the cycle structure of $\sigma$ and is discussed above (4.56). The subgroup $G(\pi)$ for a given set partition is defined in (4.54) and permutes each block of $\pi$ within itself but does not mix the different blocks. As mentioned below (4.56), the set Perms $(\pi(\sigma))$ is just the conjugacy class of $\sigma$ under $G(\pi(\sigma))$, and therefore

$$
\mathcal{P}(\sigma)=T_{\pi(\sigma)}
$$

As with the flavour projection, we now prove

$$
\mathcal{P}^{2}=\mathcal{P} \quad \mathcal{P}^{\dagger}=\mathcal{P}
$$


These follow immediately from the definition. We have

$$
\begin{aligned}
\mathcal{P}(\mathcal{P}(\sigma)) & =\frac{1}{|G(\pi(\sigma))|^{2}} \sum_{\tau \in G(\pi(\sigma))} \sum_{\mu \in G\left(\pi\left(\tau \sigma \tau^{-1}\right)\right)} \mu \tau \sigma \tau^{-1} \mu^{-1} \\
& =\frac{1}{|G(\pi(\sigma))|^{2}} \sum_{\tau \in G(\pi(\sigma))} \sum_{\mu \in G\left(\pi\left(\tau \sigma \tau^{-1}\right)\right)} \tau \tau^{-1} \mu \tau \sigma \tau^{-1} \mu^{-1} \tau \tau^{-1} \\
& =\frac{1}{|G(\pi(\sigma))|^{2}} \sum_{\tau, \tilde{\mu} \in G(\pi(\sigma))} \tau \tilde{\mu} \sigma \tilde{\mu}^{-1} \tau^{-1} \\
& =\frac{1}{|G(\pi(\sigma))|^{2}} \sum_{\tau \in G(\pi(\sigma))}|G(\pi(\sigma))| \tau \alpha \tau^{-1} \\
& =\mathcal{P}(\alpha)
\end{aligned}
$$

where in the third line, we have defined $\tilde{\mu}=\tau^{-1} \mu \tau$. The conjugation by $\tau$ takes $\mu \in$ $G\left(\pi\left(\tau \sigma \tau^{-1}\right)\right)$ to $\tilde{\mu} \in G(\pi(\sigma))$. To prove $\mathcal{P}$ is Hermitian, we note that

$$
g(\sigma, \mathcal{P}(\tau))=\frac{1}{|G(\pi(\tau))|} \sum_{\mu \in G(\pi(\tau))} \delta\left(\sigma \mu \tau^{-1} \mu^{-1}\right)
$$

is only non-zero if $\sigma, \tau$ belong to the same $\operatorname{Perms}(\pi)$. In particular, they have $\pi(\sigma)=\pi(\tau)$. Therefore

$$
\begin{aligned}
g(\sigma, \mathcal{P}(\tau)) & =\frac{1}{|G(\pi(\sigma))|} \sum_{\mu \in G(\pi(\sigma))} \delta\left(\mu^{-1} \sigma \mu \tau^{-1}\right) \\
& =\frac{1}{|G(\pi(\sigma))|} \sum_{\mu \in G(\pi(\sigma))} \delta\left(\mu \sigma \mu^{-1} \tau^{-1}\right) \\
& =g(\mathcal{P}(\sigma), \tau)
\end{aligned}
$$

Therefore the map (7.5) gives an isomorphism between the symmetrised traces of $n$ matrices with degree $(1, \ldots, 1)$ and either $\operatorname{Im} \mathcal{P}$ or $\mathbb{C}\left(S_{n}\right) / \operatorname{Ker} \mathcal{P}$. This is true when $N \geq n$. To deal with $N<n$ we have to include the finite $N$ relations as well, which is discussed later.

As $\mathcal{P}$ is satisfies (7.31), it is expressible in the standard projector form

$$
\mathcal{P}=\sum_{i}|i\rangle\langle i|
$$

for orthonormal basis states $|i\rangle$ for $\operatorname{Im}(\mathcal{P})$. These states $|i\rangle=\alpha_{i}$ belong to $\mathbb{C}\left(S_{n}\right)$, so to avoid doubling of notation, we will write this as

$$
\mathcal{P}=\sum_{i} \alpha_{i} \otimes \alpha_{i}
$$

which acts on $\sigma \in S_{n}$ via

$$
\mathcal{P}(\sigma)=\sum_{i} g\left(\alpha_{i}, \sigma\right) \alpha_{i}
$$


It is clear from (7.30) that $T_{\pi}$ spans $\operatorname{Im} \mathcal{P}$. For $\pi \neq \pi^{\prime}, \operatorname{Perms}(\pi)$ is disjoint from $\operatorname{Perms}\left(\pi^{\prime}\right)$, and therefore

$$
\begin{aligned}
g\left(T_{\pi}, T_{\pi^{\prime}}\right) & =\frac{1}{|\operatorname{Perms}(\pi)|\left|\operatorname{Perms}\left(\pi^{\prime}\right)\right|} \sum_{\substack{\sigma \in \operatorname{Perms}(\pi) \\
\tau \in \operatorname{Perms}\left(\pi^{\prime}\right)}} \delta\left(\sigma \tau^{-1}\right) \\
& =\frac{1}{|\operatorname{Perms}(\pi)|} \delta_{\pi, \pi^{\prime}}
\end{aligned}
$$

So an orthonormal basis for $\operatorname{Im} \mathcal{P}$ is given by

$$
\alpha_{\pi}=\sqrt{|\operatorname{Perms}(\pi)|} T_{\pi}
$$

and the corresponding expression for $\mathcal{P}$ is

$$
\mathcal{P}=\sum_{\pi \in \Pi(n)}|\operatorname{Perms}(\pi)| T_{\pi} \otimes T_{\pi}
$$

In section 4.3 we defined another $T_{\pi}$ as a map from 2-vector partitions to multi-symmetric functions. Composing these with the map (3.6) between multi-symmetric functions and symmetrised trace operators, we can identify $T_{\pi} \in \mathbb{C}\left(S_{n}\right)$ with these using the flavour projector $\mathcal{P}_{H}$ with $H=S_{n_{1}} \times S_{n_{2}}$.

Let $\mathbf{p} \vdash\left(n_{1}, n_{2}\right)$ be a vector partition, and $\pi$ a set partition such that $\pi\left(\left[(1,0)^{n_{1}},(0,1)^{n_{2}}\right]\right)=\mathbf{p}$, where the action of a set partition on a vector partition was given in (4.39). Then

$$
T_{\mathbf{p}}=\operatorname{Tr}\left[\mathcal{P}_{H}\left(T_{\pi}\right) \mathbb{X}\right]
$$

where $T_{\mathbf{p}}$ is the 2-matrix symmetrised trace operator given in (3.4).

Intuitively, the flavour projection and symmetrisation projectors should commute, since symmetrising a trace and renaming matrices from $Z_{i}$ to $X_{j}$ are commuting operations. Indeed

$$
\begin{aligned}
\mathcal{P} P_{H}(\sigma) & =\frac{1}{\left|G\left(\pi\left(\tau \sigma \tau^{-1}\right)\right)\right|} \frac{1}{|H|} \sum_{\substack{\tau \in H \\
\mu \in G\left(\pi\left(\tau \sigma \tau^{-1}\right)\right)}} \mu \tau \sigma \tau^{-1} \mu^{-1} \\
& =\frac{1}{|G(\pi(\sigma))||H|} \sum_{\substack{\tau \in H \\
\mu \in G\left(\pi\left(\tau \sigma \tau^{-1}\right)\right)}} \tau\left(\tau^{-1} \mu \tau\right) \sigma\left(\tau^{-1} \mu \tau\right)^{-1} \tau^{-1} \\
& =\frac{1}{|G(\pi(\sigma))||H|} \sum_{\substack{\tau \in H \\
\tilde{\mu} \in G(\pi(\sigma))}} \tau \tilde{\mu} \sigma \tilde{\mu}^{-1} \tau^{-1} \\
& =P_{H} \mathcal{P}(\sigma)
\end{aligned}
$$

$\mathcal{P}$ was first considered in [32], though a slightly different group $G(\pi)$ was used in the definition. This involves wreath products and is given in section 5.7 for an integer partition. The difference in the defining group does not affect the action of the projector. 


\subsection{Multi-symmetric function isomorphism for $n$ matrices}

In section 3.2 we described the isomorphism of $[35,36]$ between $\mathrm{U}(N)$ gauge invariant of 2 complex matrices $X_{1}$ and $X_{2}$, modulo the ideal generated by commutators, and the ring of multi-symmetric functions in 2 families of variables. This was then used to construct a basis for 2-matrix symmetrised traces that respected the finite $N$ behaviour. We now generalise this to the $n$-matrix case, and use the previous section to identify the space of multi-symmetric functions with sub-algebras of $\mathbb{C}\left(S_{n}\right)$. This will in turn allow a construction of the projector $\mathcal{P}_{N}$ that describes the interaction of the finite $N$ cut-off with the symmetrisation projector $\mathcal{P}$.

Consider the $M$ matrix variables $X_{1}, X_{2}, \cdots, X_{M}$. For each $a \in\{1, \cdots, M\}$, we have $N^{2}$ variables

$$
\left(X_{a}\right)_{i j}
$$

where $i, j \in\{1, \cdots, N\}$. Consider the ring of polynomials in these $M N^{2}$ variables. In this ring, there is an ideal generated by the elements of the commutators

$$
\left[X_{a}, X_{b}\right]_{i k}=\sum_{j}\left(X_{a}\right)_{i j}\left(X_{b}\right)_{j k}-\left(X_{b}\right)_{i j}\left(X_{a}\right)_{j k}
$$

where $a \neq b \in\{1,2, \cdots, M\}$. We can form a quotient ring from this ideal. The ring of polynomial functions in the $M$ matrix variables admits an action by $\mathcal{U} \in \mathrm{U}(N)$ (or $\operatorname{GL}(N, \mathbb{C}))$ :

$$
X_{a} \rightarrow \mathcal{U} X_{a} \mathcal{U}^{-1}
$$

The ideal generated by the commutators is invariant under the action of $\mathrm{U}(N)$, so there is a quotient ring of $\mathrm{U}(N)$ invariant polynomials. This is the ring of gauge invariants modulo commutator traces. This quotient ring of gauge invariants consists of multi-traces where any two traces differing by commutator traces define the same element of the ring. This is denoted by $A_{P}^{G}$ in Theorem 3 of [35].

There is a polynomial ring $D$ generated by $x_{i}^{a}$ for $a \in\{1, \cdots, M\}$ and $i \in\{1, \cdots, N\}$. These polynomials have an $S_{N}$ action given by

$$
x_{i}^{a} \rightarrow x_{\sigma(i)}^{a}
$$

The $S_{N}$ invariant polynomials form multi-symmetric functions in $M$ families of variables, and the ring of these functions is denoted $D^{S_{N}}$. Theorem 3 of [35] states that these two rings $D^{S_{N}}$ and $A_{P}^{G}$ are isomorphic.

To summarise, we have an isomorphism between gauge invariant polynomial functions of $M$ matrices, modulo commutator traces, and permutation invariant polynomial functions of the $M$ diagonal matrices. The map from the ring of $\mathrm{U}(N)$ gauge invariant polynomial functions of matrices, modulo the commutator trace, to the space of $S_{N}$ invariant polynomials is obtained by evaluating the gauge invariant functions on diagonal matrices. This map, denoted by $\iota$, is proved to be an isomorphism in [35, 36].

In the following, we will use a special case of this isomorphism where we have $M=n$ matrices and we consider gauge invariants containing exactly one of each matrix. 
The space of matrix invariants appearing in this special case is important for the construction of BPS states. As discussed in section 3, the construction of quarter-BPS states is based on finding the orthogonal complement to the operators which are expressible as commutator traces at finite $N$. This orthogonalisation admits a generalization to the present case of $M=n$ matrices and gauge invariants containing one matrix of each type. Using the permutation description of $n$-matrix traces given in the previous section, it can be expressed as a problem in $\mathbb{C}\left(S_{n}\right)$ or constructing the orthogonal complement of $\operatorname{Ker} \mathcal{P}+\operatorname{Ker} \mathcal{F}_{N}$.

To see this, recall that permutations in $\operatorname{Ker} \mathcal{P}$ correspond to commutator traces via (7.5), while those in $\operatorname{Ker} \mathcal{F}_{N}$ correspond to the zero operator. Therefore any permutation in $\operatorname{Ker} \mathcal{P}+\operatorname{Ker} \mathcal{F}_{N}$ is a commutator trace. It follows that

\section{Lemma 1.}

$$
\begin{gathered}
\left.\left(\mathbb{C}\left[X_{1}, X_{2}, \cdots, X_{n}\right] /\left\langle\left\{\left[X_{a}, X_{b}\right]: 1 \leq a<b \leq n\right\}\right\rangle\right)^{\mathrm{U}(N)}\right|_{(1,1, \cdots, 1)} \\
=\mathbb{C}\left(S_{n}\right) /\left(\operatorname{Ker} \mathcal{P}+\operatorname{Ker} \mathcal{F}_{N}\right)
\end{gathered}
$$

Composing these two isomorphisms gives an identification between multi-symmetric functions and the quotient space of permutations.

The ring of multi-symmetric functions in $n$ families of variables is spanned by multitraces of $n$ commuting matrices or monomials functions, denoted by $T_{\pi}$ and $M_{\pi}$ respectively. As discussed in section 7.1.3, these are labelled by set partitions $\pi \in \Pi(n)$ when the degree of each family of variables is 1 , since this is equivalent to a $n$-vector partition of $(1, \ldots, 1)$. The size $N$ of each family of variables limit the number of subsets in the $\pi$ to be less than $N$. This is denoted by $|\pi| \leq N$ and follows immediately from the definition of the $M_{\pi}$, given for 2 families of variables in (4.20).

We use the same notation $T_{\pi}$ and $M_{\pi}$ for the multi-symmetric functions and the equivalent permutations. For $T_{\pi}$, this is given in (7.28). The $M_{\pi}$ and $T_{\pi}$ are related by

$$
M_{\pi}=\sum_{\pi^{\prime}} \widetilde{C}_{\pi}^{\pi^{\prime}} T_{\pi^{\prime}} \quad T_{\pi}=\sum_{\pi^{\prime}} C_{\pi}^{\pi^{\prime}} M_{\pi^{\prime}}
$$

where the $C$ and $\widetilde{C}$ matrices are described in section 4.5 .

We now investigate the decomposition of $\mathbb{C}\left(S_{n}\right)$ in terms of the images and kernels of $\mathcal{P}$ and $\mathcal{F}_{N}$.

Lemma 2. Consider two subspaces $S_{1}, S_{2}$ of a vector space, equipped with an inner product. Let $S_{1}^{\perp}, S_{2}^{\perp}$ be the orthogonal complements to $S_{1}, S_{2}$ respectively. Let $S_{1}+S_{2}$ be the set of vectors of the form $v_{1}+v_{2}$, where $v_{1} \in S_{1}, v_{2} \in S_{2}$. It is a standard result that

$$
\left(S_{1}+S_{2}\right)^{\perp}=S_{1}^{\perp} \cap S_{2}^{\perp}
$$

which is stated as "The orthogonal complement of a sum of vector spaces is the intersection of orthogonal complements". 
Proof. Suppose $w \in S_{1}^{\perp} \cap S_{2}^{\perp}$, then

$$
v_{1} \cdot w=v_{2} \cdot w=0
$$

for all $v_{1} \in S_{1}, v_{2} \in S_{2}$. It follows that $w \cdot\left(v_{1}+v_{2}\right)=w \cdot v_{1}+w \cdot v_{2}=0$. So we conclude that $w \in\left(S_{1}+S_{2}\right)^{\perp}$.

Conversely, suppose $w \notin S_{1}^{\perp} \cap S_{2}^{\perp}$, then $w \notin S_{i}^{\perp}$ for $i=1$ or 2 . This means there is some $v \in S_{i}$, such that $w \cdot v \neq 0$. But $v \in S_{1}+S_{2}$, so $w \notin\left(S_{1}+S_{2}\right)^{\perp}$.

Taking $S_{1}=\operatorname{Im} \mathcal{P}$ and $S_{2}=\operatorname{Im} \mathcal{F}_{N}$, we have an orthogonal decomposition for $\mathbb{C}\left(S_{n}\right)$ with respect to $g_{n}$

\section{Lemma 2.}

$$
\mathbb{C}\left(S_{n}\right)=\left(\operatorname{Im} \mathcal{P} \cap \operatorname{Im} \mathcal{F}_{N}\right) \oplus_{g_{n}}\left(\operatorname{Ker} \mathcal{P}+\operatorname{Ker} \mathcal{F}_{N}\right)
$$

Using the fact that the monomial multi-symmetric functions form a basis, we have

$$
\operatorname{Im} \mathcal{P}=\mathcal{M}=\operatorname{Span}\left\{M_{\pi}: \pi \in \Pi(n)\right\}
$$

We will also define

$$
\begin{aligned}
& \mathcal{M}_{N}^{\leq}=\operatorname{Span}\left\{M_{\pi}: \pi \in \Pi(n),|\pi| \leq N\right\} \\
& \mathcal{M}_{N}^{>}=\operatorname{Span}\left\{M_{\pi}: \pi \in \Pi(n),|\pi|>N\right\}
\end{aligned}
$$

For $n<N$, we have to consider both operators $\mathcal{F}_{N}$ and $\mathcal{P}$. They are both hermitian operators w.r.t. the $g_{n}$ inner product, but they do not commute. The space $\operatorname{Ker} \mathcal{P}+\operatorname{Ker} \mathcal{F}_{N}$, spanned by sums of vectors in $\operatorname{Ker} \mathcal{P}$ and $\operatorname{Ker} \mathcal{F}_{N}$ is in general bigger than $\operatorname{Ker} \mathcal{P}$. There is non-trivial intersection

$$
\operatorname{Im} \mathcal{P} \cap\left(\operatorname{Ker} \mathcal{P}+\operatorname{Ker} \mathcal{F}_{N}\right)
$$

The non-triviality of this intersection is reflected in the fact that some symmetrised traces can also be written as a commutator trace at finite $N$. An example of this is given in (6.26).

Since $\mathcal{P}$ is a Hermitian projector, we have the orthogonal decomposition

$$
\mathbb{C}\left(S_{n}\right)=\operatorname{Im} \mathcal{P} \oplus_{g_{n}} \operatorname{Ker} \mathcal{P}
$$

It follows that we have an orthogonal decomposition of $\operatorname{Ker} \mathcal{P}+\operatorname{Ker} \mathcal{F}_{N}$

\section{Lemma 3.}

$$
\operatorname{Ker} \mathcal{P}+\operatorname{Ker} \mathcal{F}_{N}=\left(\left(\operatorname{Ker} \mathcal{P}+\operatorname{Ker} \mathcal{F}_{N}\right) \cap \operatorname{Im} \mathcal{P}\right) \oplus_{g_{n}} \operatorname{Ker} \mathcal{P}
$$

\section{Lemma 4.}

$$
\left(\operatorname{Ker} \mathcal{P}+\operatorname{Ker} \mathcal{F}_{N}\right) \cap \operatorname{Im} \mathcal{P}=\mathcal{M}_{N}^{>}=\operatorname{Span}\left\{M_{\pi},|\pi|>N\right\}
$$

Proof. $\mathcal{M}_{N}^{>}$is exactly the subspace of $\mathcal{M}$ which is not in the image of the isomorphism $\iota$. Therefore

$$
\mathcal{M}_{N}^{>} \subset \operatorname{Ker} \mathcal{P}+\operatorname{Ker} \mathcal{F}_{N} \subset \mathbb{C}\left(S_{n}\right)
$$

Additionally $\mathcal{M}_{N}^{>} \subset \operatorname{Im} \mathcal{P}=(\operatorname{Ker} \mathcal{P})^{\perp}$. Then using Lemma 4, the result follows. 
Using Lemmas 2, 3 and 4 we have the result

\section{Theorem.}

$$
\mathbb{C}\left(S_{n}\right)=\left(\operatorname{Im} P \cap \operatorname{Im} \mathcal{F}_{N}\right) \oplus_{g_{n}} \mathcal{M}_{N}^{>} \oplus_{g_{n}} \operatorname{Ker} \mathcal{P}
$$

Using the definition of $\mathcal{P}_{N}$ in (7.2) we can also write this as

$$
\mathbb{C}\left(S_{n}\right)=\operatorname{Im} \mathcal{P}_{N} \oplus_{g_{n}} \mathcal{M}_{N}^{>} \oplus_{g_{n}} \operatorname{Ker} \mathcal{P}
$$

This gives a procedure, based on the combinatorics of multi-symmetric functions, for constructing the projector $\mathcal{P}_{N}$. This projector is built by constructing the projector for the susbspace of $\mathcal{M}$ orthogonal, with respect to the inner product $g_{n}$ on $\mathbb{C}\left(S_{n}\right)$, to $\mathcal{M}_{N}^{>}$. Since $\mathcal{M}_{N}^{\leq}+\mathcal{M}_{N}^{>}=\operatorname{Im} \mathcal{P}$, this can be built by taking the vectors in $\mathcal{M}_{N}^{\leq}$and subtracting vectors in $\mathcal{M}_{N}^{>}$to ensure the orthogonality. The construction of $\operatorname{Im} \mathcal{P} \cap \operatorname{Im} \mathcal{F}_{N}$ uses the following elements:

- Vectors $M_{\pi}$ in $\mathbb{C}\left(S_{n}\right)$ labelled by set partitions, spanning $\operatorname{Im} \mathcal{P}$.

- Finite $N$ cut-off implemented using the set partition labels: the condition $|\pi| \leq N$ which defines $\mathcal{M} \leq$

- Orthogonalization of $\mathcal{M}_{N}^{\leq}$to $\mathcal{M}_{N}^{>}$with respect to the inner product $g_{n}$.

This procedure is used in section 6 to construct quarter-BPS bases.

The result of this procedure is a vector subspace of $\operatorname{Im} \mathcal{F}_{N}$, and therefore the orthogonalisation can equivalently be done using the inner product $g_{n, \widehat{N}}$ for any $\widehat{N} \geq N$, as on these permutations the $g_{n}$ and $g_{n, \widehat{N}}$ inner products are the same.

We now give a construction of the projector $\mathcal{P}_{N}$ that captures this process. The formula for this is given in (7.73).

\subsection{Finite $N$ symmetrisation operator on $\mathbb{C}\left(S_{n}\right)$}

We now construct the projector $\mathcal{P}_{N}$ onto $\operatorname{Im} \mathcal{P} \cap \operatorname{Im} \mathcal{F}_{N}$ and prove it has the projector properties

$$
\left(\mathcal{P}_{N}\right)^{2}=\mathcal{P}_{N} \quad\left(\mathcal{P}_{N}\right)^{\dagger}=\mathcal{P}_{N}
$$

and commutes with the flavour projector

$$
\mathcal{P}_{N} \mathcal{P}_{H}=\mathcal{P}_{H} \mathcal{P}_{N}
$$

Before producing $\mathcal{P}_{N}$, we give an alternative formula for $\mathcal{P}$, the large $N$ symmetrisation projector. Using (7.40) and substituting using (7.48), we have

$$
\mathcal{P}=\sum_{\pi, \pi^{\prime}, \pi^{\prime \prime} \in \Pi(n)}|\operatorname{Perms}(\pi)| C_{\pi}^{\pi^{\prime}} C_{\pi}^{\pi^{\prime \prime}} M_{\pi^{\prime}} \otimes M_{\pi^{\prime \prime}}=\sum_{\pi, \pi^{\prime} \in \Pi(n)}\left(C D C^{T}\right)_{\pi^{\prime}}^{\pi} M_{\pi} \otimes M_{\pi^{\prime}}
$$

where $D$ is the diagonal matrix

$$
D_{\pi^{\prime}}^{\pi}=|\operatorname{Perms}(\pi)| \delta_{\pi, \pi^{\prime}}
$$


To understand the appearance of $C D C^{T}$, note that this is the inverse metric on the subspace $\operatorname{Im}(\mathcal{P})$ of $\mathbb{C}\left(S_{n}\right)$.

We know $g$ is a positive definite inner product on the entirety of $\mathbb{C}\left(S_{n}\right)$. It is therefore also a positive definite inner product on the subspace $\operatorname{Im} \mathcal{P}$. Hence there is an inverse metric on this subspace, which we call $G$. Using (7.48) we have

$$
\begin{aligned}
g\left(M_{\pi}, M_{\pi^{\prime}}\right) & =\sum_{\pi_{1}, \pi_{2} \in \Pi(n)} \widetilde{C}_{\pi}^{\pi_{1}} \widetilde{C}_{\pi^{\prime}}^{\pi_{2}} g\left(T_{\pi_{1}}, T_{\pi_{2}}\right) \\
& =\sum_{\pi_{1} \in \Pi(n)} \frac{\widetilde{C}_{\pi}^{\pi_{1}} \widetilde{C}_{\pi^{\prime}}^{\pi_{1}}}{\left|\operatorname{Perms}\left(\pi_{1}\right)\right|} \\
& =\left(\widetilde{C}^{T} D^{-1} \widetilde{C}\right)_{\pi^{\prime}}^{\pi}
\end{aligned}
$$

Since $C$ and $\widetilde{C}$ are inverses of each other, this implies

$$
G\left(M_{\pi}, M_{\pi^{\prime}}\right)=\left(C D C^{T}\right)_{\pi^{\prime}}^{\pi}
$$

We can therefore write

$$
\mathcal{P}=\sum_{\pi, \pi^{\prime} \in \Pi(n)}\left(C D C^{T}\right)_{\pi^{\prime}}^{\pi} M_{\pi} \otimes M_{\pi^{\prime}}=\sum_{\pi, \pi^{\prime} \in \Pi(n)} G\left(M_{\pi}, M_{\pi^{\prime}}\right) M_{\pi} \otimes M_{\pi^{\prime}}
$$

This form for a projector is a generalisation of (7.35) to a basis of the image that is not orthonormal. We now find a basis for $\operatorname{Im} \mathcal{P}_{N}$, and can use the form above to write down $\mathcal{P}_{N}$.

At finite $N$, we want to project onto the orthogonal complement of $\mathcal{M}_{N}^{>}$within $\operatorname{Im} \mathcal{P}$. The $M_{\pi}$ with $|\pi| \leq N$ do not suffice for this as they are not orthogonal to $M_{\pi}$ with $|\pi|>N$; we need to orthogonalise them first.

As already noted, $g$ is an inner product on any subspace of $\mathbb{C}\left(S_{n}\right)$. This time the relevant subspace is $\mathcal{M}_{N}^{>}$. This means that the matrix of inner products $g\left(M_{\pi}, M_{\pi^{\prime}}\right)$ for $|\pi|,\left|\pi^{\prime}\right|>N$ is invertible and has an inverse metric that we call $G^{>}$. Note that $G^{>}$is distinct to $G$, which is the inverse inner product on $\operatorname{Im} \mathcal{P}=\mathcal{M}$. Practically, the difference is

$$
\begin{array}{lr}
\left.\sum_{\substack{\pi^{\prime} \in \Pi(n) \\
\pi^{\prime} \in \Pi(n) \\
\left|\pi^{\prime}\right|>N}} g\left(M_{\pi}, M_{\pi^{\prime}}\right) G\left(M_{\pi^{\prime}}, M_{\pi^{\prime \prime}}\right)=\delta_{\pi \pi^{\prime \prime}} \quad \pi, M_{\pi^{\prime}}\right) G^{>}\left(M_{\pi^{\prime}}, M_{\pi^{\prime \prime}}\right)=\delta_{\pi \pi^{\prime \prime}} \text { unrestricted } \\
& |\pi|,\left|\pi^{\prime \prime}\right|>N
\end{array}
$$

We can use $G^{>}$to construct a basis for $\mathcal{M}_{N}^{\leq}$, labelled by those set partitions with $|\pi| \leq N$.

$$
\bar{M}_{\pi}=M_{\pi}-\sum_{\substack{\pi_{1}, \pi_{2} \in \Pi(n) \\\left|\pi_{1}\right|,\left|\pi_{2}\right|>N}} G^{>}\left(M_{\pi_{1}}, M_{\pi_{2}}\right) g\left(M_{\pi}, M_{\pi_{1}}\right) M_{\pi_{2}}
$$

The simplest way of looking at this is to notice that the second term is using a projector of the form (7.67) applied to $M_{\pi}$. This is the projector

$$
\mathcal{P}^{>}=\sum_{\substack{\pi_{1}, \pi_{2} \in \Pi(n) \\\left|\pi_{1}\right|,\left|\pi_{2}\right|>N}} G^{>}\left(M_{\pi_{1}}, M_{\pi_{2}}\right)\left(M_{\pi_{1}} \otimes M_{\pi_{2}}\right)
$$


that orthogonally projects onto $\mathcal{M}_{N}^{>}$. The construction of $\bar{M}_{\pi}$ just applies $1-\mathcal{P}^{>}$to $M_{\pi}$ to produce something orthogonal to $\mathcal{M}_{N}^{>}$while remaining in $\operatorname{Im} \mathcal{P}$. We can now use the $\bar{M}_{\pi}$ to define the finite $N$ symmetrisation projector. Again, we need to produce a new inverse metric $G \leq$ on the space spanned by $\bar{M}_{\pi}$. This satisfies

$$
\sum_{\substack{\pi^{\prime} \in \Pi(n) \\\left|\pi^{\prime}\right| \leq N}} g\left(\bar{M}_{\pi}, \bar{M}_{\pi^{\prime}}\right) G^{\leq}\left(\bar{M}_{\pi^{\prime}}, \bar{M}_{\pi^{\prime \prime}}\right)=\delta_{\pi \pi^{\prime \prime}} \quad|\pi|,\left|\pi^{\prime \prime}\right| \leq N
$$

Using this, we construct the finite $N$ symmetrisation projector

$$
\mathcal{P}_{N}=\sum_{\substack{\pi, \pi^{\prime} \in \Pi(n) \\|\pi|,\left|\pi^{\prime}\right| \leq N}} G^{\leq}\left(\bar{M}_{\pi}, \bar{M}_{\pi^{\prime}}\right)\left(\bar{M}_{\pi} \otimes \bar{M}_{\pi^{\prime}}\right)
$$

We now prove the properties (7.61) and (7.62) for $\mathcal{P}_{N}$.

$\mathcal{P}_{N}$ is a projector. To prove this, we act with the square of the projector

$$
\begin{aligned}
\mathcal{P}_{N} \mathcal{P}_{N}(\alpha)= & \mathcal{P}_{N} \sum_{\substack{\pi_{1}, \pi_{2} \in \Pi(n) \\
\left|\pi_{1}\right|,\left|\pi_{2}\right| \leq N}} G^{\leq}\left(\bar{M}_{\pi_{1}}, \bar{M}_{\pi_{2}}\right) \bar{M}_{\pi_{1}} g\left(\bar{M}_{\pi_{2}}, \alpha\right) \\
= & \sum_{\substack{\pi_{1}, \pi_{2}, \pi_{3}, \pi_{4} \in \Pi(n) \\
\left|\pi_{1}\right|,\left|\pi_{2}\right|,\left|\pi_{3}\right|,\left|\pi_{4}\right| \leq N}}^{\leq} G^{\leq}\left(\bar{M}_{\pi_{3}}, \bar{M}_{\pi_{4}}\right) G^{\leq}\left(\bar{M}_{\pi_{1}}, \bar{M}_{\pi_{2}}\right) g\left(\bar{M}_{\pi_{4}}, \bar{M}_{\pi_{1}}\right) g\left(\bar{M}_{\pi_{2}}, \alpha\right) \bar{M}_{\pi_{3}} \\
= & \sum_{\substack{\pi_{1}, \pi_{2}, \pi_{3} \in \Pi(n) \\
\left|\pi_{1}\right|,\left|\pi_{2}\right|,\left|\pi_{3}\right| \leq N}} \delta\left(\pi_{1}, \pi_{3}\right) G \leq\left(\bar{M}_{\pi_{1}}, \bar{M}_{\pi_{2}}\right) g\left(\bar{M}_{\pi_{2}}, \alpha\right) \bar{M}_{\pi_{3}} \\
& =\sum_{\substack{\pi_{1}, \pi_{2} \in \Pi(n) \\
\left|\pi_{1}\right|,\left|\pi_{2}\right| \leq N}} G \leq\left(\bar{M}_{\pi_{1}}, \bar{M}_{\pi_{2}}\right) \bar{M}_{\pi_{1}} g\left(\bar{M}_{\pi_{2}}, \alpha\right) \\
& =\mathcal{P}_{N}(\alpha)
\end{aligned}
$$

where we have used (7.72) to get from the second to third line.

$\mathcal{P}_{N}$ is hermitian. This follows from the symmetry between $\pi$ and $\pi^{\prime}$ in (7.73)

$$
\begin{aligned}
g\left(\alpha, \mathcal{P}_{N}(\beta)\right) & =\sum_{\substack{\pi_{1}, \pi_{2} \in \Pi(n) \\
\left|\pi_{1}\right|,\left|\pi_{2}\right| \leq N}} G^{\leq}\left(\bar{M}_{\pi_{1}}, \bar{M}_{\pi_{2}}\right) g\left(\bar{M}_{\pi_{2}}, \beta\right) g\left(\alpha, \bar{M}_{\pi_{1}}\right) \\
& =g\left(\mathcal{P}_{N}(\alpha), \beta\right)
\end{aligned}
$$

$\mathcal{P}_{N}$ commutes with $\mathcal{P}_{\boldsymbol{H}}$. This relies on some smaller results. We start with

$$
\sigma^{-1} T_{\pi} \sigma=T_{\sigma(\pi)}
$$

where we define $\sigma(\pi)$ as the set partition obtained by substituting $i \rightarrow \sigma(i)$ in the set partition $\pi$. It is useful to recall the fact that $\sigma^{-1} \mu \sigma$ is the permutation obtained by the substitution $i \rightarrow \sigma(i)$ in the cycle decomposition of $\mu$, and therefore

$$
\operatorname{Perms}(\sigma(\pi))=\sigma^{-1} \operatorname{Perms}(\pi) \sigma=\left\{\sigma^{-1} \mu \sigma: \mu \in \operatorname{Perms}(\pi)\right\}
$$


It follows that

$$
\begin{aligned}
\sigma^{-1} T_{\pi} \sigma & =\frac{1}{|\operatorname{Perms}(\pi)|} \sum_{\mu \in \operatorname{Perms}(\pi)} \sigma^{-1} \mu \sigma \\
& =\frac{1}{|\operatorname{Perms}(\sigma(\pi))|} \sum_{\tilde{\mu} \in \operatorname{Perms}(\sigma(\pi))} \tilde{\mu} \\
& =T_{\sigma(\pi)}
\end{aligned}
$$

We also observe that

$$
C_{\pi_{2}}^{\pi_{1}}=C_{\sigma\left(\pi_{2}\right)}^{\sigma\left(\pi_{1}\right)} \quad \widetilde{C}_{\pi_{2}}^{\pi_{1}}=\widetilde{C}_{\sigma\left(\pi_{2}\right)}^{\sigma\left(\pi_{1}\right)}
$$

This is because the incidence relations of the poset of set partitions are unchanged when we go from set partitions of $\{1,2, \cdots, n\}$ to set partitions of $\{\sigma(1), \cdots, \sigma(n)\}$.

It follows from (7.76) and (7.79) that

$$
\begin{aligned}
\sigma^{-1} M_{\pi} \sigma & =\sum_{\pi^{\prime} \in \Pi(n)} \widetilde{C}_{\pi}^{\pi^{\prime}} \sigma^{-1} T_{\pi^{\prime}} \sigma \\
& =\sum_{\pi^{\prime} \in \Pi(n)} \widetilde{C}_{\sigma(\pi)}^{\sigma\left(\pi^{\prime}\right)} T_{\sigma\left(\pi^{\prime}\right)} \\
& =\sum_{\pi^{\prime} \in \Pi(n)} \widetilde{C}_{\sigma(\pi)}^{\pi^{\prime}} T_{\pi^{\prime}} \\
& =M_{\sigma(\pi)}
\end{aligned}
$$

where in going from the 2nd to 3rd line we have reparameterised the sum by $\pi^{\prime} \rightarrow \sigma\left(\pi^{\prime}\right)$, which clearly just permutes the set partitions in $\Pi(n)$ among each other.

It is immediate from (7.6) that

$$
g\left(\sigma \alpha \sigma^{-1}, \sigma \beta \sigma^{-1}\right)=g(\alpha, \beta)
$$

Applying this to $\alpha=M_{\pi}, \beta=M_{\pi^{\prime}}$ and using (7.80), we have

$$
g\left(M_{\sigma(\pi)}, M_{\sigma\left(\pi^{\prime}\right)}\right)=g\left(M_{\pi}, M_{\pi^{\prime}}\right)
$$

We would like to show that $G$ also has this property. To see this, note that $G$ is defined by the property (7.68), so we need to show that the matrix $G\left(M_{\sigma(\pi)}, M_{\sigma\left(\pi^{\prime}\right)}\right)$ satisfies the same relation.

$$
\begin{aligned}
\sum_{\pi^{\prime} \in \Pi(n)} g\left(M_{\pi}, M_{\pi^{\prime}}\right) G\left(M_{\sigma\left(\pi^{\prime}\right)}, M_{\sigma\left(\pi^{\prime \prime}\right)}\right) & =\sum_{\pi^{\prime} \in \Pi(n)} g\left(M_{\pi}, M_{\sigma^{-1}\left(\pi^{\prime}\right)}\right) G\left(M_{\pi^{\prime}}, M_{\sigma\left(\pi^{\prime \prime}\right)}\right) \\
& =\sum_{\pi^{\prime} \in \Pi(n)} g\left(M_{\sigma(\pi)}, M_{\pi^{\prime}}\right) G\left(M_{\pi^{\prime}}, M_{\sigma\left(\pi^{\prime \prime}\right)}\right) \\
& =\delta_{\sigma(\pi) \sigma\left(\pi^{\prime \prime}\right)} \\
& =\delta_{\pi \pi^{\prime \prime}}
\end{aligned}
$$

Therefore

$$
G\left(M_{\sigma(\pi)}, M_{\sigma\left(\pi^{\prime}\right)}\right)=G\left(M_{\pi}, M_{\pi^{\prime}}\right)
$$


Next note that $|\pi|=|\sigma(\pi)|$, so when changing variables from $\pi$ to $\sigma(\pi)$, the restrictions $|\pi|>N$ or $|\pi| \leq N$ are maintained. This means we can repeat the steps in (7.83) but using $G^{>}$or $G^{\leq}$instead. Hence

$$
G^{>}\left(M_{\sigma(\pi)}, M_{\sigma\left(\pi^{\prime}\right)}\right)=G^{>}\left(M_{\pi}, M_{\pi^{\prime}}\right) \quad G^{\leq}\left(M_{\sigma(\pi)}, M_{\sigma\left(\pi^{\prime}\right)}\right)=G^{\leq}\left(M_{\pi}, M_{\pi^{\prime}}\right)
$$

where $\pi, \pi^{\prime}$ satisfy the appropriate constraints on their length for the two operations.

Using the definition (7.70), as well as (7.80), (7.81) and (7.85)

$$
\begin{aligned}
\sigma^{-1} \bar{M}_{\pi} \sigma & =\sigma^{-1} M_{\pi} \sigma-\sum_{\substack{\pi_{1}, \pi_{2} \in \Pi(n) \\
\left|\pi_{1}\right|,\left|\pi_{2}\right|>N}} G^{>}\left(M_{\pi_{1}}, M_{\pi_{2}}\right) g\left(M_{\pi}, M_{\pi_{1}}\right) \sigma^{-1} M_{\pi_{2}} \sigma \\
& =M_{\sigma(\pi)}-\sum_{\substack{\pi_{1}, \pi_{2} \in \Pi(n) \\
\left|\pi_{1}\right|,\left|\pi_{2}\right|>N}} G^{>}\left(M_{\pi_{1}}, M_{\pi_{2}}\right) g\left(M_{\pi}, M_{\pi_{1}}\right) M_{\sigma\left(\pi_{2}\right)} \\
& =M_{\sigma(\pi)}-\sum_{\substack{\pi_{1}, \pi_{2} \in \Pi(n) \\
\left|\pi_{1}\right|,\left|\pi_{2}\right|>N}}^{>>}\left(M_{\pi_{1}}, M_{\sigma^{-1}\left(\pi_{2}\right)}\right) g\left(M_{\pi}, M_{\pi_{1}}\right) M_{\pi_{2}} \\
& =M_{\sigma(\pi)}-\sum_{\substack{\pi_{1}, \pi_{2} \in \Pi(n) \\
\left|\pi_{1}\right|,\left|\pi_{2}\right|>N}}^{>} G^{>}\left(M_{\sigma\left(\pi_{1}\right)}, M_{\pi_{2}}\right) g\left(M_{\pi}, M_{\pi_{1}}\right) M_{\pi_{2}} \\
& =M_{\sigma(\pi)}-\sum_{\substack{\pi_{1}, \pi_{2} \in \Pi(n) \\
\left|\pi_{1}\right|,\left|\pi_{2}\right|>N}}^{>>}\left(M_{\pi_{1}}, M_{\pi_{2}}\right) g\left(M_{\pi}, M_{\sigma^{-1}\left(\pi_{1}\right)}\right) M_{\pi_{2}} \\
& =M_{\sigma(\pi)}-\sum_{\substack{\pi_{1}, \pi_{2} \in \Pi(n) \\
\left|\pi_{1}\right|,\left|\pi_{2}\right|>N}}^{>} G^{>}\left(M_{\pi_{1}}, M_{\pi_{2}}\right) g\left(M_{\sigma(\pi)}, M_{\pi_{1}}\right) M_{\pi_{2}} \\
& =\bar{M}_{\sigma(\pi)} \quad
\end{aligned}
$$

We can now prove that $\mathcal{P}_{N}$ and $\mathcal{P}_{H}$ commute

$$
\begin{aligned}
& \mathcal{P}_{N} P_{H}(\alpha)=\frac{1}{|H|} \sum_{\sigma \in H} \sum_{\substack{\pi_{1}, \pi_{2} \in \Pi(n) \\
\left|\pi_{1}\right|,\left|\pi_{2}\right| \leq N}} G^{\leq}\left(\bar{M}_{\pi_{2}}, \bar{M}_{\pi_{1}}\right) \bar{M}_{\pi_{2}} g\left(\bar{M}_{\pi_{1}}, \sigma \alpha \sigma^{-1}\right) \\
& =\frac{1}{|H|} \sum_{\sigma \in H} \sum_{\substack{\pi_{1}, \pi_{2} \in \Pi(n) \\
\left|\pi_{1}\right|,\left|\pi_{2}\right| \leq N}} G^{\leq}\left(\bar{M}_{\pi_{2}}, \bar{M}_{\pi_{1}}\right) \bar{M}_{\pi_{2}} g\left(\sigma^{-1} \bar{M}_{\pi_{1}} \sigma, \alpha\right) \\
& =\frac{1}{|H|} \sum_{\sigma \in H} \sum_{\substack{\pi_{1}, \pi_{2} \in \Pi(n) \\
\left|\pi_{1}\right|,\left|\pi_{2}\right| \leq N}} G^{\leq}\left(\bar{M}_{\pi_{2}}, \bar{M}_{\pi_{1}}\right) \bar{M}_{\pi_{2}} g\left(\bar{M}_{\sigma\left(\pi_{1}\right)}, \alpha\right) \\
& =\frac{1}{|H|} \sum_{\sigma \in H} \sum_{\substack{\pi_{1}, \pi_{2} \in \Pi(n) \\
\left|\pi_{1}\right|,\left|\pi_{2}\right| \leq N}} G^{\leq}\left(\bar{M}_{\pi_{2}}, \bar{M}_{\sigma^{-1}\left(\pi_{1}\right)}\right) \bar{M}_{\pi_{2}} g\left(\bar{M}_{\pi_{1}}, \alpha\right) \\
& =\frac{1}{|H|} \sum_{\sigma \in H} \sum_{\substack{\pi_{1}, \pi_{2} \in \Pi(n) \\
\left|\pi_{1}\right|,\left|\pi_{2}\right| \leq N}} G^{\leq}\left(\bar{M}_{\sigma\left(\pi_{2}\right)}, \bar{M}_{\pi_{1}}\right) \bar{M}_{\pi_{2}} g\left(\bar{M}_{\pi_{1}}, \alpha\right) \\
& =\frac{1}{|H|} \sum_{\sigma \in H} \sum_{\substack{\pi_{1}, \pi_{2} \in \Pi(n) \\
\left|\pi_{1}\right|,\left|\pi_{2}\right| \leq N}} G^{\leq}\left(\bar{M}_{\pi_{2}}, \bar{M}_{\pi_{1}}\right) \bar{M}_{\sigma^{-1}\left(\pi_{2}\right)} g\left(\bar{M}_{\pi_{1}}, \alpha\right)
\end{aligned}
$$




$$
\begin{aligned}
& =\frac{1}{|H|} \sum_{\sigma \in H} \sum_{\substack{\pi_{1}, \pi_{2} \in \Pi(n) \\
\left|\pi_{1}\right|,\left|\pi_{2}\right| \leq N}} G \leq\left(\bar{M}_{\pi_{2}}, \bar{M}_{\pi_{1}}\right) \sigma \bar{M}_{\pi_{2}} \sigma^{-1} g\left(\bar{M}_{\pi_{1}}, \alpha\right) \\
& =P_{H} \mathcal{P}_{N}(\alpha)
\end{aligned}
$$

We can interpret this in words as follows. Recall that permutations $\sigma$ generate gauge invariant operators via (7.5). Imagine we start with the $n$-flavour gauge invariant operator generated by $\sigma$, and then symmetrise the traces, and map that to symmetrised permutations. This means applying $\mathcal{P}$ then setting

$$
\begin{gathered}
Z_{1}, Z_{2}, \ldots, Z_{n_{1}} \rightarrow X \\
Z_{n_{1}+1}, Z_{n_{1}+2}, \ldots, Z_{n} \rightarrow Y
\end{gathered}
$$

On the other hand, we could specialise the $n$-flavour gauge invariants to 2-flavour gauge invariants before projecting to symmetrised traces. Intuitively, thinking about traces, we don't see any reason for a difference between the two orders of arriving at symmetrised traces of two matrices. So we expect the two projectors to commute. Indeed they do as shown above.

\section{Hidden 2D topology: permutation TFT2 for the counting and corre- lators at weak coupling}

The connection between delta functions on symmetric group algebras and two-dimensional topological field theories (TFT2) is explained in [32]. We will give the delta function formulae and explain the TFT2 defects.

Lemma 8.1. In the problem of gauge invariants of $n$ matrices, each occuring once, the counting of symmetrised traces at large $N$ is given by

$$
\sum_{\alpha \in S_{n}} \delta\left(\mathcal{P}\left(\alpha^{-1}\right) \mathcal{P}(\alpha)\right)
$$

Proof. The symmetrised traces form the image of the hermitian projector $\mathcal{P}$. So the dimension of the space of symmetrised traces is calculated as

$$
\begin{aligned}
\operatorname{Dim}(\operatorname{Im} \mathcal{P}) & =\sum_{\alpha \in S_{n}} g(\alpha, \mathcal{P}(\alpha)) \\
& =\sum_{\alpha \in S_{n}} g(\alpha, \mathcal{P}(\mathcal{P}(\alpha))) \\
& =\sum_{\alpha \in S_{n}} g(\mathcal{P}(\alpha), \mathcal{P}(\alpha)) \\
& =\sum_{\alpha \in S_{n}} \delta\left(\mathcal{P}\left(\alpha^{-1}\right) \mathcal{P}(\alpha)\right)
\end{aligned}
$$


Proposition 8.2. The counting of quarter-BPS operators in the large $N$ limit in the free theory is given by

$$
\sum_{\alpha \in S_{n}} \delta\left(\mathcal{P}_{H}(\alpha) \alpha^{-1}\right)
$$

where $\mathcal{P}_{H}$ is the flavour projector onto two flavours with $H=S_{n_{1}} \times S_{n_{2}}$ as described in $(7.21)$.

Proof. We know that permutations can be used to construct 2-matrix gauge invariants and there is an equivalence up to conjugation by $H$, given in (7.20). Using Burnside's Lemma to count the free field operators, we have

$$
\frac{1}{|H|} \sum_{\gamma \in H} \sum_{\alpha \in S_{n}} \delta\left(\gamma \alpha \gamma^{-1} \alpha^{-1}\right)=\sum_{\alpha \in S_{n}} \delta\left(\mathcal{P}_{H}(\alpha) \alpha^{-1}\right)
$$

This is the free field counting of 2-matrix operators [32].

Proposition 8.3. The counting of 2-matrix symmetrised operators in the $\left(n_{1}, n_{2}\right)$ sector is

$$
\sum_{\alpha \in S_{n}} \delta\left(\mathcal{P}_{H} \mathcal{P}(\alpha) \mathcal{P}\left(\alpha^{-1}\right)\right)
$$

Proof. Both $\mathcal{P}, \mathcal{P}_{H}$ are hermitian with respect to the standard inner product on $\mathbb{C} S_{n}$ and they commute, so they can be simultaneously diagonalised. The dimension of the intersection of their images is equal to the trace of their product

$$
\begin{aligned}
\sum_{\alpha \in S_{n}} g\left(\alpha, \mathcal{P} \mathcal{P}_{H}(\alpha)\right) & =\sum_{\alpha \in S_{n}} g\left(\alpha, \mathcal{P}^{2} \mathcal{P}_{H}(\alpha)\right) \\
& =\sum_{\alpha \in S_{n}} g\left(\mathcal{P}(\alpha), \mathcal{P} \mathcal{P}_{H}(\alpha)\right) \\
& =\sum_{\alpha \in S_{n}} \delta\left(\mathcal{P}\left(\alpha^{-1}\right) \mathcal{P} P_{H}(\alpha)\right) \\
& =\sum_{\alpha \in S_{n}} \delta\left(\mathcal{P}_{H} \mathcal{P}(\alpha) \mathcal{P}\left(\alpha^{-1}\right)\right)
\end{aligned}
$$

Proposition 8.4. The counting formula for the finite $N$ quarter-BPS operators is

$$
\sum_{\alpha \in S_{n}} \delta\left(\mathcal{P}_{H} \mathcal{P}_{N}(\alpha) \mathcal{P}_{N}\left(\alpha^{-1}\right)\right)
$$

Proof. Given that we have proved the projector, hermiticity, and commutativity properties of $\mathcal{P}_{N}$ and $\mathcal{P}_{H}$, we can calculate the dimension of the image of $\mathcal{P}_{N} \mathcal{P}_{H}$ by repeating the steps we had for $\mathcal{P}$ and $\mathcal{P}_{H}$

$$
\begin{aligned}
\operatorname{Dim}\left(\operatorname{Im}\left(\mathcal{P}_{N} \mathcal{P}_{H}\right)\right) & =\sum_{\alpha \in S_{n}} g\left(\alpha, \mathcal{P}_{N} \mathcal{P}_{H}(\alpha)\right) \\
& =\sum_{\alpha \in S_{n}} g\left(\alpha, \mathcal{P}_{N}^{2} \mathcal{P}_{H}(\alpha)\right)
\end{aligned}
$$




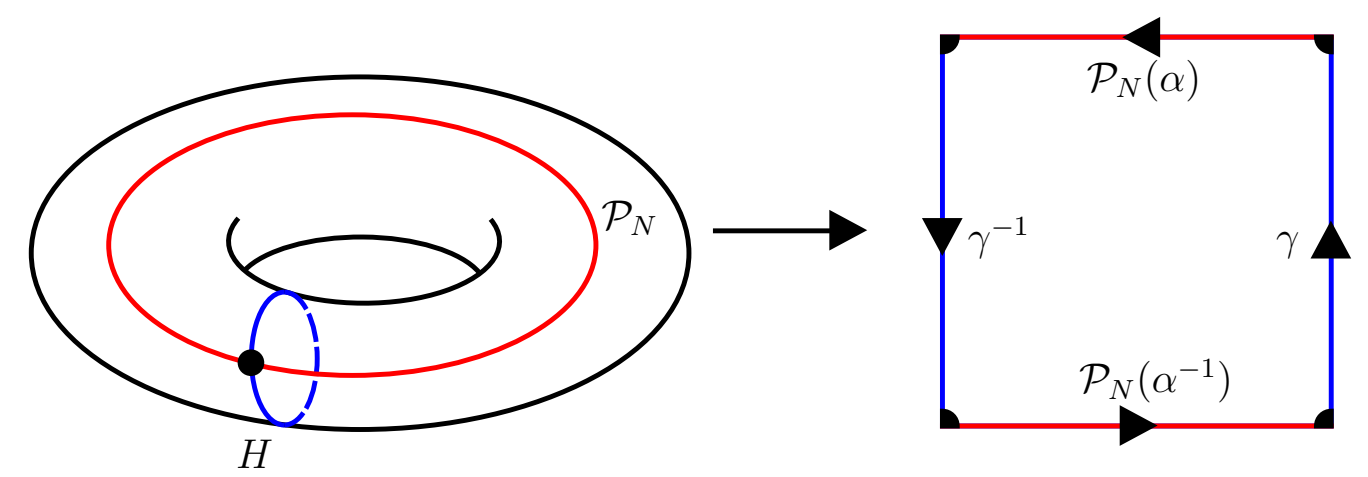

Figure 5. TFT2 partition function for finite $N$ weak coupling BPS counting.

$$
\begin{aligned}
& =\sum_{\alpha \in S_{n}} g\left(\mathcal{P}_{N}(\alpha), \mathcal{P}_{N} \mathcal{P}_{H}(\alpha)\right) \\
& =\sum_{\alpha \in S_{n}} \delta\left(\mathcal{P}_{N}\left(\alpha^{-1}\right) \mathcal{P}_{N} \mathcal{P}_{H}(\alpha)\right) \\
& =\sum_{\alpha \in S_{n}} \delta\left(\mathcal{P}_{H} \mathcal{P}_{N}(\alpha) \mathcal{P}_{N}\left(\alpha^{-1}\right)\right)
\end{aligned}
$$

Proposition 8.5. The finite $N$ two-point function for BPS states can be written as

$$
\begin{aligned}
\left\langle\mathcal{G}_{N} \mathcal{P}_{N} \operatorname{Tr}\left(\alpha_{1} X^{\otimes n_{1}} Y^{\otimes n_{2}}\right), \mathcal{G}_{N} \mathcal{P}_{N} \operatorname{Tr}\left(\alpha_{2} X^{\otimes n_{1}} Y^{\otimes n_{2}}\right)\right\rangle & \\
& =\delta\left(\mathcal{P}_{H} \mathcal{P}_{N}\left(\alpha_{1}\right) \mathcal{P}_{N}\left(\alpha_{2}^{-1}\right) \Omega_{N}^{-1}\right)
\end{aligned}
$$

This follows as in [32]. $\Omega_{N}^{-1} \mathcal{P}_{N}(\alpha)$ span the BPS states as $\alpha$ runs over $\mathbb{C}\left(S_{n}\right)$. The free field inner product is $g_{F F}(\alpha, \beta)=g\left(\alpha, \Omega_{N} \beta\right)$. The step forward in this paper is that we have an explicit construction of $\mathcal{P}_{N}$ using set partitions.

Now we will draw the TFT2 pictures corresponding to these delta function formulae. Figure 5 gives us the counting of weak coupling BPS operators. Figure 6 gives the TFT2 formulation for the 2-point function of quarter BPS operators at weak coupling. The one new ingredient in these TFT2 constructions is the $\mathcal{P}_{N}$-defect which can be associated to a circle. The defect is defined by declaring that it modifies the permutation $\alpha$ associated to that circle in the TFT2 to $\mathcal{P}_{N}(\alpha)$.

\section{Summary and outlook}

We have given a construction of quarter BPS operators in $\mathcal{N}=4$ SYM with $\mathrm{U}(N)$ gauge group, built from two matrices $X, Y$ and annihilated by the 1-loop dilatation operator of the $\mathrm{SU}(2)$ sector. The construction depends on parameters $n, N$ which are arbitrary, with $n$ being the number of $X, Y$ matrices in the operator. The construction, given in sections 6 and 7, produces an orthogonal basis of operators which obeys an SEP-compatibility condition. The labels for the basis operators include a $\mathrm{U}(2)$ Young diagram $\Lambda$ and a $\mathrm{U}(N)$ 


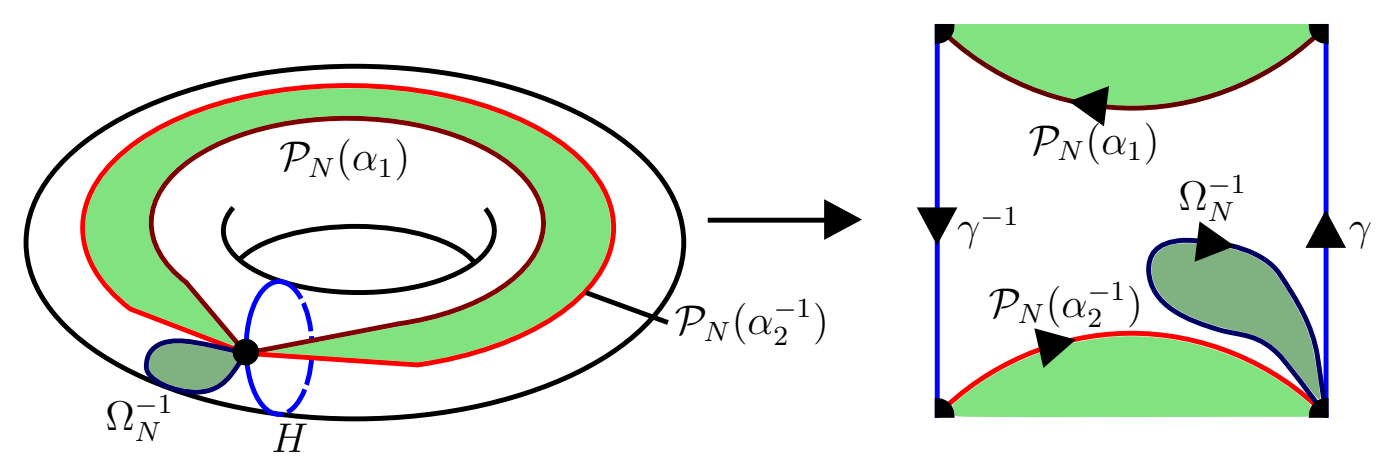

Figure 6. TFT2 partition function for finite $N$ BPS 2-point function.

Young diagram $p$, alongside multiplicity labels. The SEP-compatibility means that finite $N$ effects are captured simply by restricting the length of $p$ (defined as the length of the first column of the Young diagram) to be less than $N$. In section 5 we have given detailed formulae for the dimensions of the multiplicity spaces as a function of $\Lambda, p$.

The understanding of holographic map between the quarter-BPS sector between $\mathcal{N}=4$ SYM and $A d S_{5} \times S^{5}$ is far less well-developed than the half-BPS sector. The Young diagram labels for half-BPS states have provided valuable tools for precision mapping of states between SYM and the dual space-time. In the quarter BPS sector, there is a rich combinatoric structure involving $\Lambda, p$ and the plethysm problem underlying the multiplicities $\mathcal{M}(\Lambda, p)$, which control the structure of states. If will be fascinating to uncover the role of these structures in the dual space-time. Conceretely, reproducing the refined multiplicity formulae for specified $\Lambda, p$ from the weakly coupled gravitational dual, is an interesting problem. It has been shown [42] that three-point functions involving quarterBPS operators, alongside half-BPS operators are not renormalized, while the question of non-renormalization for more general quarter-BPS operators remains open. It will be interesting to compute correlators involving the operators constructed here and compare these with calculations involving giant gravitons and LLM geometries, generalising the successful comparisons in the half-BPS giants/geometries and fluctuations thereof, such as in $[11-14,66]$. A natural generalization of the work presented here is to develop the analogous discussion for $\mathrm{SO}(N) / \mathrm{SP}(N)$ gauge groups, building on earlier work at zero and weak coupling $[67-72,72,73]$.

\section{Acknowledgments}

SR is supported by the STFC consolidated grant ST/P000754/1 "String Theory, Gauge Theory \& Duality" and a Visiting Professorship at the University of the Witwatersrand, funded by a Simons Foundation grant (509116) awarded to the Mandelstam Institute for Theoretical Physics. We are grateful for conversations on the subject of this paper to Matt Buican, Robert de Mello Koch, Joan Simon. 


\section{A $\quad \Lambda=[3,2]$ sector}

In this and the following appendices we give explicit examples of quarter-BPS operators constructed using the algorithm presented in this paper. The code used to generate these examples has been made available on Github [74]. In this code, we take $N$ to be a large indeterminate and proceed down the left hand side of figure and along the bottom of 1 .

This appendix gives the operators in the $\Lambda=[3,2]$ sector with $M_{\Lambda}$ the highest weight state with field content $(3,2)$. Other states in the $\mathrm{U}(2)$ representation can be reached by applying the lowering operator $\mathcal{J}_{-}$.

Throughout this section we will work with $\Lambda=[3,2]$ and $M_{\Lambda}=\frac{1}{\left.\frac{1}{2}\right|_{2} ^{1}} 1$, so we will suppress this index in operator labels.

For each BPS operator, we will first present it as a sum over the free field basis (2.64) and then as a sum over symmetrised traces and commutator traces, for which we use the covariant bases discussed in section 5.2. The covariant symmetrised trace basis is

$$
\begin{aligned}
t_{[3,2]} & =\operatorname{Tr} X^{3} \operatorname{Tr} Y^{2}-2 \operatorname{Tr} X^{2} Y \operatorname{Tr} X Y+\operatorname{Tr} X^{2} \operatorname{Tr} X Y^{2} \\
t_{[3,1,1]} & =\operatorname{Tr} X^{3}(\operatorname{Tr} Y)^{2}-2 \operatorname{Tr} X \operatorname{Tr} X^{2} Y \operatorname{Tr} Y+(\operatorname{Tr} X)^{2} \operatorname{Tr} X Y^{2} \\
t_{[2,2,1]} & =\operatorname{Tr} X \operatorname{Tr} X^{2} \operatorname{Tr} Y^{2}-\operatorname{Tr} X(\operatorname{Tr} X Y)^{2} \\
t_{[2,1,1,1]} & =\operatorname{Tr} X \operatorname{Tr} X^{2}(\operatorname{Tr} Y)^{2}-2(\operatorname{Tr} X)^{2} \operatorname{Tr} X Y \operatorname{Tr} Y+(\operatorname{Tr} X)^{3} \operatorname{Tr} Y^{2}
\end{aligned}
$$

and the covariant commutator trace basis is

$$
\begin{aligned}
c_{[5]} & =\operatorname{Tr} X^{3} Y^{2}-\operatorname{Tr} X^{2} Y X Y=\operatorname{Tr} X^{2}[X, Y] Y \\
c_{[4,1]} & =\operatorname{Tr} X \operatorname{Tr} X^{2} Y^{2}-\operatorname{Tr} X \operatorname{Tr}(X Y)^{2}=\operatorname{Tr} X \operatorname{Tr} X[X, Y] Y
\end{aligned}
$$

For these two bases, the partition label describes the cycle structure of the multi-traces.

The free field operators can be written in terms of symmetrised and commutator traces

$$
\begin{aligned}
& \mathcal{O}_{\text {曰⿴囗十 }}=\frac{1}{6 \sqrt{10}}\left(3 t_{[3,2]}+t_{[3,1,1]}+4 t_{[2,2,1]}+t_{[2,1,1,1]}+6 c_{[5]}+4 c_{[4,1]}\right) \\
& \mathcal{O}_{\boxplus}=\frac{1}{6 \sqrt{2}}\left(t_{[3,1,1]}+t_{[2,2,1]}+t_{[2,1,1,1]}-3 c_{[5]}-2 c_{[4,1]}\right) \\
& \mathcal{O}_{\text {目 }, \text { odd }}=\frac{1}{2 \sqrt{30}}\left(2 t_{[3,2]}-t_{[2,1,1,1]}-4 c_{[4,1]}\right) \\
& \mathcal{O}_{\text {田, even }}=\frac{1}{2 \sqrt{5}}\left(t_{[3,1,1]}-t_{[2,2,1]}+c_{[5]}\right) \\
& \mathcal{O}_{\boxplus}=\frac{1}{6 \sqrt{2}}\left(t_{[3,1,1]}+t_{[2,2,1]}-t_{[2,1,1,1]}-3 c_{[5]}+2 c_{[4,1]}\right) \\
& \mathcal{O}_{\text {甲 }}=\frac{1}{6 \sqrt{10}}\left(-3 t_{[3,2]}+t_{[3,1,1]}+4 t_{[2,2,1]}-t_{[2,1,1,1]}+6 c_{[5]}-4 c_{[4,1]}\right)
\end{aligned}
$$

The odd/even labels for the $R=[3,1,1]$ multiplicity come from the odd/even permutations used to produce the respective traces. All other zero coupling operators are defined uniquely by $\Lambda$ and $R$. 


\section{A.1 BPS operators}

Following the algorithm, the BPS operators in the $\Lambda=[3,2]$ sector are

$$
\begin{aligned}
& S_{[2,1,1,1]}^{\mathrm{BPS}}=\frac{1}{2 \sqrt{15 P_{1}}}\left((N-2)(N-3)\left[2 N \mathcal{O}_{\nabla^{\boxplus}}-\sqrt{5}(N+3) \mathcal{O}_{\boxplus}\right]\right.
\end{aligned}
$$

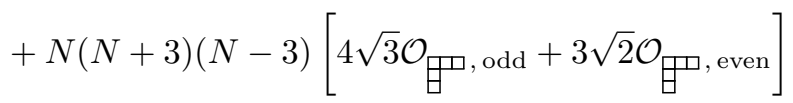

$$
\begin{aligned}
& \left.-5(N+3)(N+2)\left[\sqrt{5}(N-3) \mathcal{O}_{\boxplus}+4 N \mathcal{O}_{\text {甲 }}\right]\right) \\
& =\frac{1}{2 \sqrt{6 P_{1}}}\left[\left(N^{3}+5 N^{2}+2 N-18\right) t_{[2,1,1,1]}-4\left(N^{2}+3 N-3\right)(N+1) t_{[2,2,1]}\right. \\
& -\left(N^{2}+3 N-6\right)(N+2) t_{[3,1,1]}+3 N(N+2)(N+1) t_{[3,2]} \\
& \left.+4(2 N+9)(N+1) c_{[4,1]}-18(N+2)(N+1) c_{[5]}\right] \\
& S_{[2,2,1]}^{\mathrm{BPS}}=\frac{1}{2 \sqrt{3 P_{2}}}\left(-\sqrt{5}(N-1)(N-2)\left[2 N \mathcal{O}_{\square}-\sqrt{5}(N+3) \mathcal{O}_{\boxplus}\right]\right.
\end{aligned}
$$

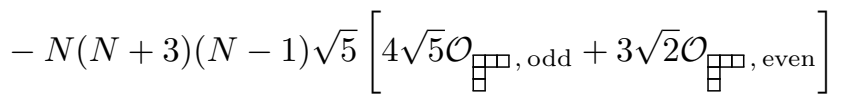

$$
\begin{aligned}
& \left.-\left(15 N^{3}+48 N^{2}+19 N+6\right) \mathcal{O}_{\text {甲 }}+2 \sqrt{5} N(3 N+8) \mathcal{O}_{\text {甲 }}\right) \\
& =\frac{1}{2 \sqrt{6 P_{2}}}\left[\left(5 N^{3}+12 N^{2}-12 N+6\right) t_{[2,1,1,1]}+2(3 N-2)(N-1) t_{[2,2,1]}\right. \\
& -\left(5 N^{3}+12 N^{2}-2 N-4\right) t_{[3,1,1]}-N\left(5 N^{2}+8 N-2\right) t_{[3,2]} \\
& \left.-4(8 N+3) c_{[4,1]}+6\left(5 N^{2}+8 N-2\right) c_{[5]}\right] \\
& S_{[3,1,1]}^{\mathrm{BPS}}=\frac{1}{\sqrt{15 P_{3}}}\left(-(2 N-1)(N-2)\left[2 N \mathcal{O}_{\square}-\sqrt{5}(N+3) \mathcal{O}_{\boxplus}\right]\right. \\
& -\sqrt{3}\left(3 N^{3}+9 N^{2}-5 N-2\right) \mathcal{O}_{\text {甲 }} \text {, odd } \\
& +3 \sqrt{2}\left(3 N^{3}+6 N^{2}-4 N+2\right) \mathcal{O}_{\text {日, }}, \text { even } \\
& \left.-(N+2)(N+1)\left[\sqrt{5} \mathcal{O}_{\boxplus}-2 \mathcal{O}_{\Xi}\right]\right) \\
& =\frac{1}{2 \sqrt{6 P_{3}}}\left[\left(N^{3}+3 N^{2}-5 N+2\right) t_{[2,1,1,1]}-4 N^{2}(N+1) t_{[2,2,1]}\right. \\
& +2\left(2 N^{3}+4 N^{2}-5 N+2\right) t_{[3,1,1]}-2 N^{2}(N+1) t_{[3,2]} \\
& \left.+8(N+1)(N-1) c_{[4,1]}+12 N(N+1) c_{[5]}\right] \\
& S_{[3,2]}^{\mathrm{BPS}}=\frac{1}{2 \sqrt{15 P_{4}}}\left(2\left(5 N^{2}-5 N+2\right) \mathcal{O}_{\boxminus}+\sqrt{5}\left(4 N^{2}+5 N-2\right) \mathcal{O}_{\boxplus}\right. \\
& +4 \sqrt{3}(N-1) \mathcal{O}_{\boxminus} \text {, odd }-3 \sqrt{2} N \mathcal{O}_{\boxminus}, \text { even }
\end{aligned}
$$

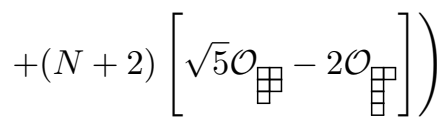




$$
=\frac{\sqrt{6}}{12 \sqrt{P_{4}}}\left[N^{2}\left(t_{[2,1,1,1]}+2 t_{[2,2,1]}+t_{[3,1,1]}+t_{[3,2]}\right)-4(N-1) c_{[4,1]}-6 N c_{[5]}\right]
$$

where the normalisation polynomials are

$$
\begin{aligned}
& P_{1}=10 N^{6}+74 N^{5}+199 N^{4}+252 N^{3}+351 N^{2}+648 N+702 \\
& P_{2}=50 N^{6}+220 N^{5}+192 N^{4}-78 N^{3}+541 N^{2}-156 N+78 \\
& P_{3}=15 N^{6}+50 N^{5}+17 N^{4}-66 N^{3}+115 N^{2}-60 N+20 \\
& P_{4}=3 N^{4}+5 N^{2}-4 N+2
\end{aligned}
$$

In [22], these operators were studied, though in the $\mathrm{SU}(N)$ gauge theory rather than the $\mathrm{U}(N)$ theory. This means all traces whose cycle structure $p \vdash n$ contained one or more $1 \mathrm{~s}$ do not contribute. In the $\Lambda=[3,2]$ sector, they found the single operator

$$
\mathcal{O}=N t_{[3,2]}-6 c_{[5]}
$$

One can check that in each of the expansions above, $t_{[3,2]}$ and $c_{[5]}$ only appear in this ratio. We have found that by expanding the gauge group to $\mathrm{U}(N)$ and allowing traces of a single matrix, there are three additional quarter-BPS operators.

\section{A.2 Norms of BPS operators}

The physical $\mathcal{F}$-weighted norms of the BPS operators are

$$
\begin{aligned}
\left|S_{[2,1,1,1]}^{\mathrm{BPS}}\right|^{2} & =\frac{(N+3)(N+2)(N+1) N^{2}(N-1)(N-2)(N-3) Q_{1}}{P_{1}} \\
\left|S_{[2,2,1]}^{\mathrm{BPS}}\right|^{2} & =\frac{(N+1) N^{2}(N-1)(N-2) Q_{1} Q_{2}}{P_{2}} \\
\left|S_{[3,1,1]}^{\mathrm{BPS}}\right|^{2} & =\frac{(N+2)(N+1) N(N-1)(N-2) Q_{2} Q_{3}}{P_{3}} \\
\left|S_{[3,2]}^{\mathrm{BPS}}\right|^{2} & =\frac{(N+2)(N+1) N^{3}(N-1) Q_{3}}{P_{4}}
\end{aligned}
$$

Where the polynomials in the numerators are

$$
\begin{aligned}
& Q_{1}=10 N^{3}+37 N^{2}+11 N-36 \\
& Q_{2}=5 N^{3}+11 N^{2}-7 N+2 \\
& Q_{3}=3 N^{3}+5 N^{2}-5 N+2
\end{aligned}
$$

We discuss the combination of linear factors and $Q$ polynomials in the numerators in section B.4.

\section{B $\Lambda=[4,2]$ sector}

We give the BPS basis for the $\Lambda=[4,2]$ sector with $M_{\Lambda}$ the highest weight state corresponding to field content $(4,2)$.

Throughout this section we will work with $\Lambda=[4,2]$ and $M_{\Lambda}=\frac{\left|\frac{11}{2 / 2}\right| 11}{\sqrt{2}}$, so we will suppress these in operator labels. 


\section{B.1 Free field covariant basis from traces}

When writing our operators as sums over the free field covariant basis (2.64), we have made a choice about how to span the free field multiplicity space for $R=[4,2],[4,1,1],[3,2,1]$, $[3,1,1,1],[2,2,1,1]$. These choices are:

$$
\begin{aligned}
& \mathcal{O}_{\boxplus, 1}=\frac{\sqrt{10}}{120}\left(-t_{[4,2]}-t_{[4,1,1]}+6 t_{[3,3]}+6 t_{[3,2,1], 1}+6 t_{[3,2,1], 2}-3 t_{[2,2,2]}-3 t_{[2,2,1,1], 1}\right. \\
& \left.+6 c_{[6], 2}+6 c_{[5,1]}-4 c_{[4,2]}+2 c_{[4,1,1]}\right) \\
& \mathcal{O}_{\boxplus, 2}=\frac{\sqrt{10}}{240}\left(-2 t_{[4,1,1]}-6 t_{[3,2,1], 1}-6 t_{[3,1,1,1]}-3 t_{[2,2,1,1], 1}+9 t_{[2,2,1,1], 2}-3 t_{[2,1,1,1,1]}\right. \\
& \left.+24 c_{[6], 1}+12 c_{[5,1]}+12 c_{[4,2]}+4 c_{[4,1,1]}\right) \\
& \mathcal{O}_{\text {日王, } 1}=\frac{\sqrt{3}}{108}\left(-t_{[4,2]}+3 t_{[4,1,1]}+6 t_{[3,3]}+6 t_{[3,2,1], 2}+6 t_{[3,1,1,1]}-3 t_{[2,2,2]}+3 t_{[2,2,1,1], 1}\right. \\
& \left.+6 t_{[2,2,1,1], 2}+12 c_{[6], 1}+6 c_{[6], 2}+6 c_{[5,1]}+2 c_{[4,2]}\right) \\
& \mathcal{O}_{\text {署, } 2}=\frac{\sqrt{15}}{1080}\left(-10 t_{[4,2]}-48 t_{[3,3]}-18 t_{[3,2,1], 1}-12 t_{[3,2,1], 2}+6 t_{[3,1,1,1]}-12 t_{[2,2,2]}\right. \\
& +3 t_{[2,2,1,1], 1}-21 t_{[2,2,1,1], 2}+9 t_{[2,1,1,1,1]} \\
& \left.-24 c_{[6], 1}-48 c_{[6], 2}+60 c_{[5,1]}+20 c_{[4,2]}+60 c_{[4,1,1]}\right) \\
& \mathcal{O}_{\text {甲 }, 1}=\frac{\sqrt{10}}{90}\left(-t_{[4,2]}+6 t_{[3,3]}-3 t_{[3,1,1,1]}+3 t_{[2,2,1,1], 1}-3 t_{[2,2,1,1], 2}-3 c_{[5,1]}+2 c_{[4,2]}\right) \\
& \mathcal{O}_{\boxplus, 2}=\frac{\sqrt{5}}{15}\left(t_{[3,2,1], 2}+t_{[2,2,2]}-c_{[6], 1}+c_{[6], 2}+c_{[4,1,1]}\right) \\
& \mathcal{O}_{\boxplus, 3}=\frac{\sqrt{5}}{90}\left(-t_{[4,1,1]}-6 t_{[3,2,1], 1}-3 t_{[3,2,1], 2}+3 t_{[2,1,1,1,1]}+12 c_{[6], 1}+12 c_{[6], 2}-4 c_{[4,1,1]}\right) \\
& \mathcal{O}_{\text {目, }, 1}=\frac{\sqrt{3}}{108}\left(-t_{[4,2]}-3 t_{[4,1,1]}+6 t_{[3,3]}-6 t_{[3,2,1], 2}+6 t_{[3,1,1,1]}+3 t_{[2,2,2]}+3 t_{[2,2,1,1], 1}\right. \\
& \left.+6 t_{[2,2,1,1], 2}-12 c_{[6], 1}-6 c_{[6], 2}+6 c_{[5,1]}+2 c_{[4,2]}\right) \\
& \mathcal{O}_{\text {目 }, 2}=\frac{\sqrt{15}}{1080}\left(-10 t_{[4,2]}-48 t_{[3,3]}+18 t_{[3,2,1], 1}+12 t_{[3,2,1], 2}+6 t_{[3,1,1,1]}+12 t_{[2,2,2]}\right. \\
& +3 t_{[2,2,1,1], 1}-21 t_{[2,2,1,1], 2}-9 t_{[2,1,1,1,1]} \\
& \left.+24 c_{[6], 1}+48 c_{[6], 2}+60 c_{[5,1]}+20 c_{[4,2]}-60 c_{[4,1,1]}\right) \\
& \mathcal{O}_{\boxplus, 1}=\frac{\sqrt{10}}{120}\left(-t_{[4,2]}+t_{[4,1,1]}+6 t_{[3,3]}-6 t_{[3,2,1], 1}-6 t_{[3,2,1], 2}+3 t_{[2,2,2]}-3 t_{[2,2,1,1], 1}\right. \\
& \left.-6 c_{[6], 2}+6 c_{[5,1]}-4 c_{[4,2]}-2 c_{[4,1,1]}\right) \\
& \mathcal{O}_{\mathbb{B}^{\oplus}, 2}=\frac{\sqrt{10}}{240}\left(2 t_{[4,1,1]}+6 t_{[3,2,1], 1}-6 t_{[3,1,1,1]}-3 t_{[2,2,1,1], 1}+9 t_{[2,2,1,1], 2}+3 t_{[2,1,1,1,1]}\right. \\
& \left.-24 c_{[6], 1}+12 c_{[5,1]}+12 c_{[4,2]}-4 c_{[4,1,1]}\right)
\end{aligned}
$$

The zero coupling operators with $R=[5,1],[3,3],[2,2,2],\left[2,1^{4}\right]$ are defined uniquely (up to a minus sign) by $\Lambda$ and $R$. We use

$$
\begin{aligned}
\mathcal{O}_{\text {Em }}= & -\frac{\sqrt{10}}{720}\left(8 t_{[4,2]}+2 t_{[4,1,1]}+24 t_{[3,3]}+30 t_{[3,2,1], 1}+6 t_{[3,1,1,1]}+12 t_{[2,2,2]}+3 t_{[2,2,1,1], 1}-21 t_{[2,2,1,1], 2}\right. \\
& \left.+3 t_{[2,1,1,1,1]}+72 c_{[6], 1}+24 c_{[6], 2}+60 c_{[5,1]}+20 c_{[4,2]}+20 c_{[4,1,1]}\right)
\end{aligned}
$$




$$
\begin{aligned}
\mathcal{O}_{\text {円 }}= & \frac{\sqrt{10}}{360}\left(t_{[4,2]}+t_{[4,1,1]}-6 t_{[3,3]}-12 t_{[3,2,1], 1}-18 t_{[3,2,1], 2}-6 t_{[3,1,1,1]}-3 t_{[2,2,2]}+6 t_{[2,2,1,1], 1}+3 t_{[2,2,1,1], 2}\right. \\
& \left.-3 t_{[2,1,1,1,1]}+30 c_{[6], 2}+30 c_{[5,1]}-20 c_{[4,2]}+10 c_{[4,1,1]}\right) \\
\mathcal{O}_{\text {田 }}= & \frac{\sqrt{10}}{360}\left(t_{[4,2]}-t_{[4,1,1]}-6 t_{[3,3]}+12 t_{[3,2,1], 1}+18 t_{[3,2,1], 2}-6 t_{[3,1,1,1]}+3 t_{[2,2,2]}+6 t_{[2,2,1,1], 1}+3 t_{[2,2,1,1], 2}\right. \\
& \left.+3 t_{[2,1,1,1,1]}-30 c_{[6], 2}+30 c_{[5,1]}-20 c_{[4,2]}-10 c_{[4,1,1]}\right) \\
\mathcal{O}_{\text {目 }}= & \frac{\sqrt{10}}{720}\left(-8 t_{[4,2]}+2 t_{[4,1,1]}-24 t_{[3,3]}+30 t_{[3,2,1], 1}-6 t_{[3,1,1,1]}+12 t_{[2,2,2]}-3 t_{[2,2,1,1], 1}+21 t_{[2,2,1,1], 2}\right. \\
& \left.+3 t_{[2,1,1,1,1]}+72 c_{[6], 1}+24 c_{[6], 2}-60 c_{[5,1]}-20 c_{[4,2]}+20 c_{[4,1,1]}\right)
\end{aligned}
$$

where the symmetrised trace combinations we use are defined by

$$
\begin{aligned}
t_{[4,2]} & =3 \operatorname{Tr} X^{4} \operatorname{Tr} Y^{2}-6 \operatorname{Tr} X^{3} Y \operatorname{Tr} X Y+2 \operatorname{Tr} X^{2} \operatorname{Tr} X^{2} Y^{2}+\operatorname{Tr} X^{2} \operatorname{Tr}(X Y)^{2} \\
t_{[4,1,1]} & =3 \operatorname{Tr} X^{4}(\operatorname{Tr} Y)^{2}-6 \operatorname{Tr} X \operatorname{Tr} X^{3} Y \operatorname{Tr} Y+2(\operatorname{Tr} X)^{2} \operatorname{Tr} X^{2} Y^{2}+(\operatorname{Tr} X)^{2} \operatorname{Tr}(X Y)^{2} \\
t_{[3,3]} & =\operatorname{Tr} X^{3} \operatorname{Tr} X Y^{2}-\left(\operatorname{Tr} X^{2} Y\right)^{2} \\
t_{[3,2,1], 1} & =\operatorname{Tr} X \operatorname{Tr} X^{3} \operatorname{Tr} Y^{2}-2 \operatorname{Tr} X \operatorname{Tr} X^{2} Y \operatorname{Tr} X Y+\operatorname{Tr} X \operatorname{Tr} X^{2} \operatorname{Tr} X Y^{2} \\
t_{[3,2,1], 2} & =\operatorname{Tr} X^{3} \operatorname{Tr} X Y \operatorname{Tr} Y-\operatorname{Tr} X \operatorname{Tr} X^{3} \operatorname{Tr} Y^{2}-\operatorname{Tr} X^{2} \operatorname{Tr} X^{2} Y \operatorname{Tr} Y+\operatorname{Tr} X \operatorname{Tr} X^{2} Y \operatorname{Tr} X Y \\
t_{[3,1,1,1]} & =\operatorname{Tr} X \operatorname{Tr} X^{3}(\operatorname{Tr} Y)^{2}-2(\operatorname{Tr} X)^{2} \operatorname{Tr} X^{2} Y \operatorname{Tr} Y+(\operatorname{Tr} X)^{3} \operatorname{Tr} X Y^{2} \\
t_{[2,2,2]} & =\left(\operatorname{Tr} X^{2}\right)^{2} \operatorname{Tr} Y^{2}-\operatorname{Tr} X^{2}(\operatorname{Tr} X Y)^{2} \\
t_{[2,2,1,1], 1} & =\left(\operatorname{Tr} X^{2} \operatorname{Tr} Y\right)^{2}-2 \operatorname{Tr} X \operatorname{Tr} X^{2} \operatorname{Tr} X Y \operatorname{Tr} Y+(\operatorname{Tr} X \operatorname{Tr} X Y)^{2} \\
t_{[2,2,1,1], 2} & =(\operatorname{Tr} X \operatorname{Tr} X Y)^{2}-(\operatorname{Tr} X)^{2} \operatorname{Tr} X^{2} \operatorname{Tr} Y^{2} \\
t_{[2,1,1,1,1]} & =(\operatorname{Tr} X)^{2} \operatorname{Tr} X^{2}(\operatorname{Tr} Y)^{2}-2(\operatorname{Tr} X)^{3} \operatorname{Tr} X Y \operatorname{Tr} Y+(\operatorname{Tr} X)^{4} \operatorname{Tr} Y^{2}
\end{aligned}
$$

along with the commutators

$$
\begin{aligned}
c_{[6], 1} & =\operatorname{Tr} X^{4} Y^{2}-\operatorname{Tr} X^{3} Y X Y=\operatorname{Tr} X^{3}[X, Y] Y \\
c_{[6], 2} & =\operatorname{Tr} X^{3} Y X Y-\operatorname{Tr}\left(X^{2} Y\right)^{2}=\operatorname{Tr} X^{2}[X, Y] Y^{2} \\
c_{[5,1]} & =\operatorname{Tr} X \operatorname{Tr} X^{3} Y^{2}-\operatorname{Tr} X \operatorname{Tr} X^{2} Y X Y=\operatorname{Tr} X \operatorname{Tr} X^{2}[X, Y] Y \\
c_{[4,2]} & =\operatorname{Tr} X^{2} \operatorname{Tr} X^{2} Y^{2}-\operatorname{Tr} X^{2} \operatorname{Tr}(X Y)^{2}=\operatorname{Tr} X^{2} \operatorname{Tr} X[X, Y] Y \\
c_{[4,1,1]} & =(\operatorname{Tr} X)^{2} \operatorname{Tr} X^{2} Y^{2}-(\operatorname{Tr} X)^{2} \operatorname{Tr}(X Y)^{2}=(\operatorname{Tr} X)^{2} \operatorname{Tr} X[X, Y] Y
\end{aligned}
$$

These are respectively the covariant symmetrised trace and commutator trace bases for the $\Lambda=[4,2]$ sector with $M_{\Lambda}$ the highest weight state, as discussed in section 5.2.

\section{B.2 Quarter-BPS basis}

We now give the end result of the construction algorithm for quarter-BPS operators in the $\Lambda=[4,2]$ sector. The operators in this section are very lengthy to write out, so in the interests of brevity we only express them as a sum of free field operators. An expression in terms of trace can be found by substituting (B.1)-(B.15). 
For $p=[3,2,1]$ and $[2,2,1,1]$ there are two BPS operators. For these, we have chosen the multiplicity space basis using the alternative orthogonalisation algorithm of section 6.10, beginning with the choice of free field multiplicities in (B.5)-(B.7) and (B.10)(B.11) respectively.

We present the operators starting from the longest partition $p=[2,1,1,1,1]$ and progressing to the shortest, $p=[4,2]$.

$$
\begin{aligned}
& S_{[2,1,1,1,1]}^{\mathrm{BPS}}=\frac{1}{6 \sqrt{3 P_{0}}}\left(( N - 1 ) ( N - 3 ) ( N - 4 ) \left[3 \sqrt{3}(N-2)\left\{N \mathcal{O}_{\text {巴س⿴囗十 }}-(N+4) \mathcal{O}_{\boxplus \square, 2}\right\}\right.\right. \\
& \left.-\sqrt{2} N(N+4)\left\{2 \sqrt{5} \mathcal{O}_{\text {目两, } 1}-11 \mathcal{O}_{\text {目 }, 2}\right\}-2 \sqrt{3}(N+4)(N+3)\left\{\mathcal{O}_{\text {甲, }}, 1+4 \sqrt{2} \mathcal{O}_{\text {甲 }}, 3\right\}\right] \\
& +\sqrt{2} N(N+4)(N+3)(N-1)(N-4)\left[10 \sqrt{5} \mathcal{O}_{\text {目, }, 1}+29 \mathcal{O}_{\text {目, }, 2}\right] \\
& +(N+4)(N+3)(N+2)\left[10 \sqrt{3}(N-3)(N-4) \mathcal{O}_{\text {田 }}\right. \\
& \left.\left.+3 \sqrt{3}(N-1)(N-4)\left\{2 \mathcal{O}_{\mathbb{\boxplus}^{,}}+13 \mathcal{O}_{\text {甲 }}, 2\right\}+65 \sqrt{3} N(N-1) \mathcal{O}_{\text {目 }}\right]\right)
\end{aligned}
$$

where the normalisation polynomial is

$$
\begin{aligned}
P_{0}= & 195 N^{10}+2298 N^{9}+9767 N^{8}+17008 N^{7}+21041 N^{6}+74974 N^{5}+135005 N^{4} \\
& -144704 N^{3}-399936 N^{2}-62976 N+707328
\end{aligned}
$$

For $p=[2,2,1,1]$ there is a two-dimensional multiplicity space. The first operator is

$$
\begin{aligned}
& S_{[2,2,1,1], 1}^{\mathrm{BPS}}=\frac{1}{6 \sqrt{30 P_{1}}}\left(-20 \sqrt{3} N(N+1)(N-2)(N-3) P_{1,1} \mathcal{O}_{\text {巴س }}\right. \\
& +\sqrt{3}(N-3) P_{1,2}\left[3(N+1)(N-2) \mathcal{O}_{\boxplus \boxplus, 1}+5(N+3)(N-2) \mathcal{O}_{\boxplus}-12 \sqrt{2}(N+3)(N+1) \mathcal{O}_{\boxplus}, 2\right] \\
& +2 \sqrt{3}(N+1)(N-3) P_{1,3}\left[3(N-2) \mathcal{O}_{\boxplus, 2}+8 \sqrt{2}(N+3) \mathcal{O}_{\text {甲, }}{ }^{3}\right] \\
& +\sqrt{10} N(N+1) P_{1,4}\left[(N-3) \mathcal{O}_{\text {四, }, 1}-5(N+3) \mathcal{O}_{\text {即, }, 1}\right] \\
& -2 \sqrt{2}(N+1)\left[5 N(N-3) P_{1,5} \mathcal{O}_{\text {四, }}+\sqrt{6}(N+3)(N-3) P_{1,6} \mathcal{O}_{\text {甲, }},+5 N(N+3) P_{1,7} \mathcal{O}_{\text {目, }}\right] \\
& +5 \sqrt{3}(N+3)(N+2)(N+1) P_{1,8}\left[5(N-3) \mathcal{O}_{\text {由 }}+3(N-1) \mathcal{O}_{\mathbb{\boxplus}^{, 1}}\right] \\
& \left.+10 \sqrt{3}(N+3)(N+2)(N+1)\left[3 P_{1,9} \mathcal{O}_{\text {田, }}-26 N P_{1,10} \mathcal{O}_{\text {目 }}\right]\right)
\end{aligned}
$$

where the normalisation and coefficient polynomials are

$$
\begin{aligned}
P_{1}= & 1254825 N^{16}+25236900 N^{15}+212913135 N^{14}+949347864 N^{13} \\
& +2265287922 N^{12}+2296326096 N^{11}-483268806 N^{10}-64991400 N^{9} \\
& +7717590681 N^{8}+4250132076 N^{7}-14563157385 N^{6}-5596987632 N^{5} \\
& +20300164460 N^{4}+5660498272 N^{3}-5514459136 N^{2}+14594125824 N \\
& +12396386304
\end{aligned}
$$




$$
\begin{aligned}
P_{1,1} & =78 N^{4}+180 N^{3}-411 N^{2}-510 N+788 \\
P_{1,2} & =195 N^{5}+1149 N^{4}+687 N^{3}-3927 N^{2}-1552 N+4448 \\
P_{1,3} & =195 N^{5}+1257 N^{4}+801 N^{3}-5871 N^{2}-3656 N+9024 \\
P_{1,4} & =975 N^{5}+6177 N^{4}+3891 N^{3}-27411 N^{2}-16176 N+40544 \\
P_{1,5} & =507 N^{5}+3225 N^{4}+2037 N^{3}-14487 N^{2}-8664 N+21632 \\
P_{1,6} & =195 N^{5}+1041 N^{4}+573 N^{3}-1983 N^{2}+552 N-128 \\
P_{1,7} & =1443 N^{5}+9129 N^{4}+5745 N^{3}-40335 N^{2}-23688 N+59456 \\
P_{1,8} & =117 N^{4}+720 N^{3}+1041 N^{2}+240 N+992 \\
P_{1,9} & =429 N^{5}+2247 N^{4}+1215 N^{3}-3585 N^{2}+2056 N-2112 \\
P_{1,10} & =54 N^{3}+273 N^{2}+120 N-572
\end{aligned}
$$

The second operator is

$$
\begin{aligned}
& S_{[2,2,1,1], 2}^{\mathrm{BPS}}=\frac{1}{3 \sqrt{6 P_{2}}}\left(\sqrt{3}(N-2)(N-3) N P_{2,1} \mathcal{O}_{\text {日皿 }}\right. \\
& -\sqrt{3}(N-3) P_{2,2}\left[3(N+1)(N-2) \mathcal{O}_{\boxplus, 1}+5(N+3)(N-2) \mathcal{O}_{\boxplus}-12 \sqrt{2}(N+3)(N+1) \mathcal{O}_{\boxplus}, 2\right] \\
& +\sqrt{3}(N-3) P_{2,3}\left[3(N-2) \mathcal{O}_{\boxplus \mathbb{m}, 2}+8 \sqrt{2}(N+3) \mathcal{O}_{\mathbb{\boxplus}}, 3\right] \\
& +\sqrt{10} N P_{2,4}\left[(N-3) \mathcal{O}_{\text {目, }}-5(N+3) \mathcal{O}_{\text {目,1 }}\right]-\sqrt{2}(N-3) N P_{2,5} \mathcal{O}_{\text {四, }, 2} \\
& +2 \sqrt{3}(N+3)(N-3) P_{2,6} \mathcal{O}_{\text {甲, }}+\sqrt{2} N(N+3) P_{2,7} \mathcal{O}_{\text {目, }}
\end{aligned}
$$

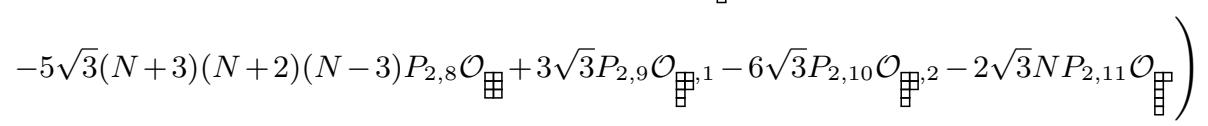

where the normalisation and coefficient polynomials are

$$
\begin{aligned}
P_{2}= & 64575225 N^{16}+1221543180 N^{15}+9292923450 N^{14}+34312809600 N^{13} \\
& +49747071546 N^{12}-49520811024 N^{11}-212528733480 N^{10}+81502221096 N^{9} \\
& +872883407025 N^{8}+609873915684 N^{7}-949480261506 N^{6}-778095650280 N^{5} \\
& +986491220724 N^{4}+591265527264 N^{3}-532623199736 N^{2}-150593123520 N \\
& +181872634752 \\
P_{2,1}= & 135 N^{5}+423 N^{4}+999 N^{3}+1653 N^{2}+1716 N+74 \\
P_{2,2}= & 351 N^{5}+1485 N^{4}-783 N^{3}-3669 N^{2}+5448 N-1832 \\
P_{2,3}= & 189 N^{6}+903 N^{5}-429 N^{4}-4851 N^{3}-1590 N^{2}+98 N-1320 \\
P_{2,4}= & 27 N^{6}-30 N^{5}-1560 N^{4}-5250 N^{3}-4959 N^{2}-3420 N-808 \\
P_{2,5}= & 675 N^{6}+2589 N^{5}-7527 N^{4}-35553 N^{3}-24606 N^{2}-13386 N-7192 \\
P_{2,6}= & 1593 N^{6}+8247 N^{5}+2379 N^{4}-22659 N^{3}+5526 N^{2}+14562 N-8648 \\
P_{2,7}= & 135 N^{6}+3189 N^{5}+23673 N^{4}+69447 N^{3}+74574 N^{2}+55014 N+8968 \\
P_{2,8}= & 2835 N^{5}+17493 N^{4}+21549 N^{3}-19317 N^{2}-10044 N+15464 \\
P_{2,9}= & 10035 N^{8}+100587 N^{7}+320580 N^{6}+201774 N^{5}-613761 N^{4}-529313 N^{3} \\
& +665098 N^{2}+243952 N-359952
\end{aligned}
$$




$$
\begin{aligned}
P_{2,10}= & 1131 N^{7}+10440 N^{6}+29667 N^{5}+13182 N^{4}-54074 N^{3}-45886 N^{2}+22026 N \\
& +24264 \\
P_{2,11}= & 2280 N^{6}+24384 N^{5}+95505 N^{4}+166002 N^{3}+120739 N^{2}+22034 N-11694
\end{aligned}
$$

For $p=[3,1,1,1]$ the operator is

$$
\begin{aligned}
& S_{[3,1,1,1]}^{\mathrm{BPS}}=\frac{1}{18 \sqrt{2 P_{3}}}\left(-3 N(N-2)(N-3) P_{3,1} \mathcal{O}_{\text {घس }}\right. \\
& +6(N-3) P_{3,2}\left[3(N+1)(N-2) \mathcal{O}_{\boxplus \rightarrow, 1}+5(N+3)(N-2) \mathcal{O}_{\boxplus}-12 \sqrt{2}(N+3)(N+1) \mathcal{O}_{\boxplus}, 2\right] \\
& +3(N-3) P_{3,3}\left[3(N-2) \mathcal{O}_{\text {田, } 2}+8 \sqrt{2}(N+3) \mathcal{O}_{\text {甲 }}, 3\right]
\end{aligned}
$$

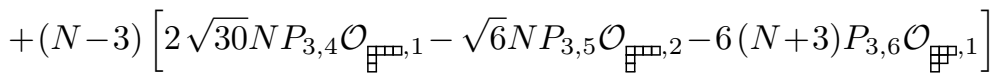

$$
\begin{aligned}
& +2 \sqrt{30} P_{3,7} \mathcal{O}_{\text {目, }, 1}-\sqrt{6} P_{3,8} \mathcal{O}_{\text {目, }} \\
& \left.-3(N+2)\left[10(N+3)(N-3) P_{3,9} \mathcal{O}_{\text {田 }}-6 P_{3,10} \mathcal{O}_{\text {甲, }}-3 P_{3,11} \mathcal{O}_{\text {甲, }}+P_{3,12} \mathcal{O}_{\text {目 }}\right]\right)
\end{aligned}
$$

where the normalisation and coefficient polynomials are

$$
\begin{aligned}
P_{3}= & 93476025 N^{16}+1612393695 N^{15}+11013446394 N^{14}+34526289987 N^{13} \\
& +29660697936 N^{12}-98498965581 N^{11}-203072674968 N^{10}+154945270125 N^{9} \\
& +449766055695 N^{8}-624364696710 N^{7}-1246035300318 N^{6}+1119952316004 N^{5} \\
& +1953728842580 N^{4}-1114329042600 N^{3}-1086753482680 N^{2}+1691309503680 N \\
& +1297828640736 \\
P_{3,1}= & 7155 N^{5}+22752 N^{4}-21231 N^{3}-76512 N^{2}+21066 N+63020 \\
P_{3,2}= & 270 N^{5}+1728 N^{4}+1287 N^{3}-6762 N^{2}-4278 N+9380 \\
P_{3,3}= & 2025 N^{6}+14460 N^{5}+19239 N^{4}-46512 N^{3}-80274 N^{2}+42292 N+71520 \\
P_{3,4}= & 2295 N^{6}+16458 N^{5}+22254 N^{4}-51987 N^{3}-91314 N^{2}+47394 N+80900 \\
P_{3,5}= & 24435 N^{6}+175044 N^{5}+235749 N^{4}-555432 N^{3}-971334 N^{2}+506028 N+861760
\end{aligned}
$$

$$
\begin{aligned}
& P_{3,6}=135 N^{6}+1524 N^{5}+4881 N^{4}+2712 N^{3}-8046 N^{2}-1476 N+3520 \\
& P_{3,7}=18630 N^{8}+168027 N^{7}+436488 N^{6}-22071 N^{5}-1221552 N^{4}-330750 N^{3}
\end{aligned}
$$$$
+1226756 N^{2}-644796 N-1298232
$$

$$
\begin{aligned}
P_{3,8}= & 37260 N^{8}+400629 N^{7}+1309611 N^{6}+387381 N^{5}-4795443 N^{4}-5190456 N^{3} \\
& +4201270 N^{2}+6554016 N+1298232
\end{aligned}
$$

$P_{3,9}=135 N^{5}+609 N^{4}-1257 N^{3}-9444 N^{2}-12438 N-5860$

$P_{3,10}=525 N^{6}+3840 N^{5}+6681 N^{4}-8262 N^{3}-49724 N^{2}-91728 N-41832$

$P_{3,11}=6825 N^{6}+61005 N^{5}+175743 N^{4}+113439 N^{3}-265222 N^{2}-407694 N-160596$

$P_{3,12}=22575 N^{6}+198375 N^{5}+553953 N^{4}+307269 N^{3}-994562 N^{2}-1589994 N-649116$ 
For $p=[2,2,2]$ the operator is

$$
\begin{aligned}
& S_{[2,2,2]}^{\mathrm{BPS}}=\frac{1}{36 \sqrt{P_{4}}}\left(30 \sqrt{2} N(N-2) P_{4,1} \mathcal{O}_{\text {甲四 }}\right.
\end{aligned}
$$

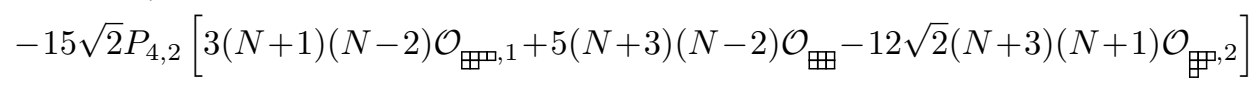

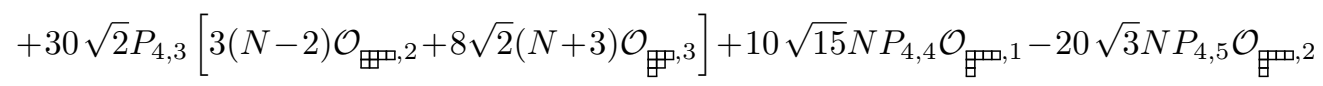

$$
\begin{aligned}
& +60 \sqrt{2}(N+3) P_{4,6} \mathcal{O}_{\text {甲, }}+10 \sqrt{3} N\left[\sqrt{5} P_{4,7} \mathcal{O}_{\text {四, } 1}+2 P_{4,8} \mathcal{O}_{\text {困 }, 2}\right]+3 \sqrt{2} P_{4,9} \mathcal{O}_{\text {田 }} \\
& \left.+45 \sqrt{2} P_{4,10} \mathcal{O}_{\text {甲, }}-90 \sqrt{2} P_{4,11} \mathcal{O}_{\text {田 }}-30 \sqrt{2} N P_{4,12} \mathcal{O}_{\text {目 }}\right)
\end{aligned}
$$

where the normalisation and coefficient polynomials are

$$
\begin{aligned}
P_{4}= & 149226300 N^{14}+2094533640 N^{13}+10660893948 N^{12}+20470965300 N^{11} \\
& -2209082715 N^{10}-23656646682 N^{9}+108969897216 N^{8}+185022077310 N^{7} \\
& -186235972937 N^{6}-216166001512 N^{5}+413959581308 N^{4}+246958572128 N^{3} \\
& -287690109584 N^{2}-143358681600 N+276161485248 \\
P_{4,1}= & 135 N^{5}-18 N^{4}-15 N^{3}-1022 N^{2}-76 N-816 \\
P_{4,2}= & 864 N^{5}+2430 N^{4}-5973 N^{3}-6119 N^{2}+17876 N-12228 \\
P_{4,3}= & 243 N^{6}+924 N^{5}-1152 N^{4}-3670 N^{3}+5307 N^{2}+2256 N-2988 \\
P_{4,4}= & 108 N^{6}+402 N^{5}-1065 N^{4}-2588 N^{3}+9471 N^{2}+3376 N+276 \\
P_{4,5}= & 945 N^{6}+3576 N^{5}-5586 N^{4}-16186 N^{3}+34863 N^{2}+13520 N-8412 \\
P_{4,6}= & 1971 N^{6}+7512 N^{5}-8238 N^{4}-27854 N^{3}+28821 N^{2}+13552 N-27444 \\
P_{4,7}= & 906 N^{5}+7485 N^{4}+18394 N^{3}+9099 N^{2}-14000 N-13164 \\
P_{4,8}= & 285 N^{5}+5955 N^{4}+34507 N^{3}+63369 N^{2}+19738 N+7536 \\
P_{4,9}= & 48060 N^{7}+429834 N^{6}+1227525 N^{5}+919710 N^{4}-762363 N^{3}-208286 N^{2} \\
& +1345500 N+946584 \\
P_{4,10}= & 3702 N^{6}+29949 N^{5}+68544 N^{4}+7491 N^{3}-79610 N^{2}+45780 N+78984 \\
P_{4,11}= & 699 N^{6}+5163 N^{5}+8795 N^{4}-9599 N^{3}-20952 N^{2}+21736 N+26328 \\
P_{4,12}= & 1605 N^{5}+14460 N^{4}+42159 N^{3}+36288 N^{2}-16754 N-19428
\end{aligned}
$$

For $p=[3,2,1]$ there is a two-dimensional multiplicity space. The first operator is

$$
\begin{aligned}
& S_{[3,2,1], 1}^{\mathrm{BPS}}=\frac{1}{45 \sqrt{P_{5}}}\left(30 \sqrt{10}(N-2) N P_{5,1} \mathcal{O}_{\text {巴س }}-6 \sqrt{10}(N-2) P_{5,2}\left[3(N+1) \mathcal{O}_{\text {四, }, 1}+5(N+3) \mathcal{O}_{\text {田 }}\right]\right. \\
& -18 \sqrt{10}(N-2) P_{5,3} \mathcal{O}_{\boxplus, 2}-20 \sqrt{3} N\left[P_{5,4} \mathcal{O}_{\text {田, } 1}-\sqrt{5} P_{5,5} \mathcal{O}_{\text {四, }}\right]-12 \sqrt{10} P_{5,6} \mathcal{O}_{\text {甲, }} \\
& -6 \sqrt{5} P_{5,7} \mathcal{O}_{\boxplus, 2}+3 \sqrt{5} P_{5,8} \mathcal{O}_{\text {甲 }}, 3-20 \sqrt{3} N\left[P_{5,9} \mathcal{O}_{\text {目,1 }}-\sqrt{5} P_{5,10} \mathcal{O}_{\text {目 }, 2}\right] \\
& \left.-6 \sqrt{10}(N+2) P_{5,11}\left[5 \mathcal{O}_{\text {田 }}+3 \mathcal{O}_{\text {田 }}\right]-6 \sqrt{10}(N+2)\left[3 P_{5,12} \mathcal{O}_{\mathbb{\Xi}^{, 2}}-5 N P_{5,13} \mathcal{O}_{\text {目 }}\right]\right)
\end{aligned}
$$


where the normalisation and coefficient polynomials are

$$
\begin{aligned}
P_{5}= & 1329483780 N^{14}+13761404280 N^{13}+47552297508 N^{12}+41944792356 N^{11} \\
& -43156801080 N^{10}+23239162764 N^{9}-47497601127 N^{8}-299164340106 N^{7} \\
& +683116078397 N^{6}+45647911732 N^{5}-883683643044 N^{4}+341394177280 N^{3} \\
& +617090703216 N^{2}-378227252672 N+179121262144 \\
P_{5,1}= & 3708 N^{5}+2172 N^{4}-19509 N^{3}+17427 N^{2}+9416 N-13724 \\
P_{5,2}= & 1656 N^{5}+5619 N^{4}-10194 N^{3}-12393 N^{2}+36194 N-24704 \\
P_{5,3}= & 5076 N^{6}+23490 N^{5}-14985 N^{4}-85957 N^{3}+116006 N^{2}+32240 N-75024 \\
P_{5,4}= & 11808 N^{6}+54255 N^{5}-34545 N^{4}-194501 N^{3}+255813 N^{2}+75970 N-174752 \\
P_{5,5}= & 12492 N^{6}+57498 N^{5}-36627 N^{4}-207175 N^{3}+274254 N^{2}+80120 N-184816 \\
P_{5,6}= & 6462 N^{7}+45960 N^{6}+66285 N^{5}-117979 N^{4}-124298 N^{3}+345680 N^{2} \\
& +16132 N-99960 \\
P_{5,7}= & 16326 N^{7}+99666 N^{6}+144600 N^{5}-54717 N^{4}-52871 N^{3}+89526 N^{2} \\
& +116 N+579336 \\
P_{5,8}= & 165978 N^{7}+1065798 N^{6}+1496280 N^{5}-1235731 N^{4}-1949513 N^{3}+8498 N^{2} \\
& +679228 N+177528 \\
P_{5,9}= & 14061 N^{5}+85773 N^{4}+110389 N^{3}-97565 N^{2}-111014 N+4008 \\
P_{5,10}= & 15126 N^{5}+66642 N^{4}-35467 N^{3}-293389 N^{2}+81536 N+220812 \\
P_{5,11}= & 1137 N^{5}+5721 N^{4}-3097 N^{3}-35915 N^{2}-21618 N-12024 \\
P_{5,12}= & 6462 N^{5}+40026 N^{4}+56743 N^{3}-30825 N^{2}-44698 N+8016 \\
P_{5,13}= & 4332 N^{4}+26304 N^{3}+32807 N^{2}-32861 N-35466
\end{aligned}
$$

The second operator is

$$
\begin{aligned}
& S_{[3,2,1], 2}^{\mathrm{BPS}}=\frac{1}{18 \sqrt{P_{6}}}\left(-3(N-2) N P_{6,1} \mathcal{O}_{\text {四 }}-3(N-2) P_{6,2}\left[3(N+1) \mathcal{O}_{\text {甲 }, 1}+5(N+3) \mathcal{O}_{\text {田 }}\right]\right.
\end{aligned}
$$

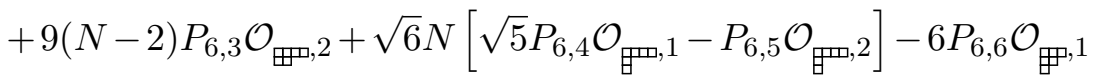

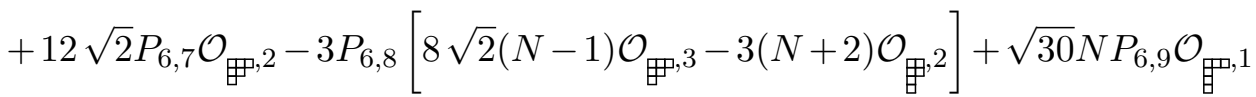

$$
\begin{aligned}
& \left.-\sqrt{6} N P_{6,10} \mathcal{O}_{\text {目 }, 2}+3(N+2) P_{6,11}\left[5 \mathcal{O}_{\text {田 }}+3 \mathcal{O}_{\text {甲 }, 1}\right]-3 N(N+2) P_{6,12} \mathcal{O}_{\text {目 }}\right)
\end{aligned}
$$

where the normalisation and coefficient polynomials are

$$
\begin{aligned}
P_{6}= & 433202580 N^{14}+4164719976 N^{13}+11536183026 N^{12}-111051000 N^{11} \\
& -29053464768 N^{10}+10364014080 N^{9}+31360792437 N^{8}-51088773768 N^{7} \\
& +37140544622 N^{6}-29831349568 N^{5}+55411748788 N^{4}-79360524160 N^{3} \\
& +66216685440 N^{2}-31168716800 N+6758052160
\end{aligned}
$$




$$
\begin{aligned}
P_{6,1}= & 864 N^{5}+7734 N^{4}-29931 N^{3}+34329 N^{2}-2260 N-8780 \\
P_{6,2}= & 5724 N^{5}+7509 N^{4}-30912 N^{3}+37572 N^{2}-28648 N+10144 \\
P_{6,3}= & 4104 N^{6}+12552 N^{5}-15267 N^{4}-24025 N^{3}+50968 N^{2}-18276 N-4944 \\
P_{6,4}= & 2484 N^{6}+11871 N^{5}-7131 N^{4}-54710 N^{3}+93012 N^{2}-18048 N-20032 \\
P_{6,5}= & 22248 N^{6}+85140 N^{5}-74325 N^{4}-290915 N^{3}+524952 N^{2}-127020 N-94960 \\
P_{6,6}= & 54810 N^{7}+273690 N^{6}+107331 N^{5}-533633 N^{4}+371678 N^{3}-35050 N^{2}-241112 N \\
& +130440 \\
P_{6,7}= & 7830 N^{7}+45693 N^{6}+36684 N^{5}-104202 N^{4}-1453 N^{3}+78398 N^{2}-91900 N+46392 \\
P_{6,8}= & 516 N^{5}+5496 N^{4}+12187 N^{3}-4448 N^{2}-12124 N+4416 \\
P_{6,9}= & 3717 N^{5}+23415 N^{4}+29524 N^{3}-26804 N^{2}-15272 N+2208 \\
P_{6,10}= & 16416 N^{5}+68640 N^{4}-93347 N^{3}-401726 N^{2}+197500 N+132600 \\
P_{6,11}= & 2685 N^{5}+12423 N^{4}+5150 N^{3}-17908 N^{2}+8976 N-6624 \\
P_{6,12}= & 6918 N^{4}+41334 N^{3}+46861 N^{2}-49160 N-18420
\end{aligned}
$$

For $p=[4,1,1]$ the operator is

$$
\begin{aligned}
& S_{[4,1,1]}^{\mathrm{BPS}}=\frac{1}{6 \sqrt{P_{7}}}\left((N-2) N P_{7,1} \mathcal{O}_{\text {四 }}-(N-2) P_{7,2}\left[3(N+1) \mathcal{O}_{\text {巴⿴囗十 } 1}+5(N+3) \mathcal{O}_{\text {田 }}\right]\right. \\
& -3(N-2) P_{7,3} \mathcal{O}_{\text {巴止 }, 2}+\sqrt{30} P_{7,4} \mathcal{O}_{\text {四, } 1}+\sqrt{6} P_{7,5} \mathcal{O}_{\text {四, } 2}-12(N+3)\left[P_{7,6} \mathcal{O}_{\text {甲, }, 1}+\sqrt{2} P_{7,7} \mathcal{O}_{\text {甲, }}\right]
\end{aligned}
$$

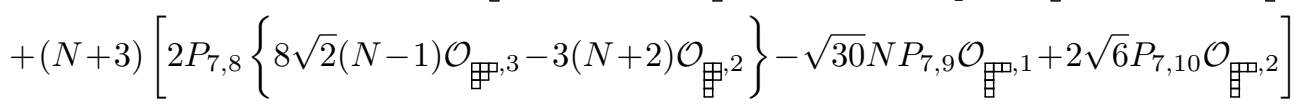

$$
\begin{aligned}
& \left.-(N+3)(N+2) P_{7,11}\left[5 \mathcal{O}_{\mathbb{\boxplus}}+3 \mathcal{O}_{\mathbb{P}^{, 1}}\right]+2(N+3)(N+2) P_{7,12} \mathcal{O}_{\text {目 }}\right)
\end{aligned}
$$

where the normalisation and coefficient polynomials are

$$
\begin{aligned}
P_{7}= & 1691280 N^{14}+14469840 N^{13}+34933194 N^{12}-15345720 N^{11} \\
& -97734483 N^{10}+108829584 N^{9}+94236018 N^{8}-365252412 N^{7} \\
& +332214736 N^{6}+23494544 N^{5}-188670784 N^{4}+59358800 N^{3} \\
& +76067360 N^{2}-55528000 N+47136640 \\
P_{7,1}= & 2052 N^{5}+2592 N^{4}-7293 N^{3}+4232 N^{2}+1320 N-2240 \\
P_{7,2}= & 108 N^{5}+477 N^{4}-348 N^{3}-770 N^{2}+1254 N-772 \\
P_{7,3}= & 612 N^{6}+3210 N^{5}+939 N^{4}-7568 N^{3}+5760 N^{2}+692 N-2472 \\
P_{7,4}= & 1296 N^{7}+6029 N^{6}+1473 N^{5}-11350 N^{4}+9484 N^{3}-4114 N^{2}-3428 N+3440 \\
P_{7,5}= & 648 N^{7}+4186 N^{6}+1473 N^{5}-12472 N^{4}+8280 N^{3}+6672 N^{2}-4760 N-3440 \\
P_{7,6}= & 13 N^{5}-7 N^{4}+84 N^{3}-129 N^{2}+222 N-40
\end{aligned}
$$




$$
\begin{aligned}
P_{7,7} & =15 N^{5}+86 N^{4}-194 N^{3}+278 N^{2}-178 N+284 \\
P_{7,8} & =69 N^{4}+222 N^{3}+112 N^{2}-193 N-62 \\
P_{7,9} & =97 N^{4}+316 N^{3}+62 N^{2}-306 N-164 \\
P_{7,10} & =263 N^{5}+410 N^{4}-1216 N^{3}-953 N^{2}+1060 N+860 \\
P_{7,11} & =15 N^{4}+60 N^{3}-262 N^{2}-146 N-244 \\
P_{7,12} & =222 N^{4}+726 N^{3}+74 N^{2}-725 N-430
\end{aligned}
$$

For $p=[3,3]$ the operator is

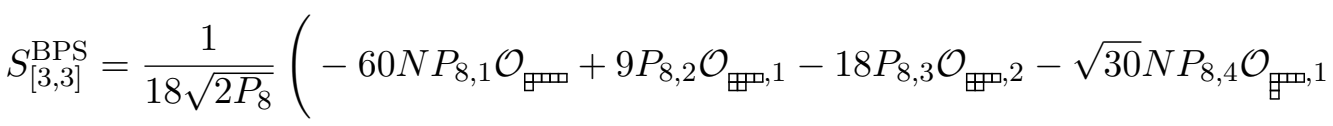

$$
\begin{aligned}
& -10 \sqrt{6} N P_{8,5} \mathcal{O}_{\text {困 }, 2}-3 P_{8,6} \mathcal{O}_{\text {田 }}+12 P_{8,7} \mathcal{O}_{\text {甲 }, 1}-12 \sqrt{2} P_{8,8} \mathcal{O}_{\text {甲 }, 2} \\
& -6 P_{8,9}\left[8 \sqrt{2}(N-1) \mathcal{O}_{\text {甲, }}-3(N+2) \mathcal{O}_{\text {甲田 }}\right]+\sqrt{6} N\left[\sqrt{5} P_{8,10} \mathcal{O}_{\text {目, }}-10 P_{8,11} \mathcal{O}_{\text {目, }}\right] \\
& \left.-3(N+2) P_{8,12}\left[5 \mathcal{O}_{\text {田 }}+3 \mathcal{O}_{\text {田 }}, 1\right]-60 N(N+2) P_{8,13} \mathcal{O}_{\text {目 }}\right)
\end{aligned}
$$

where the normalisation and coefficient polynomials are

$$
\begin{aligned}
P_{8}= & 64152 N^{12}+209952 N^{11}-137241 N^{10}-640440 N^{9}+908640 N^{8} \\
& -322236 N^{7}-116124 N^{6}-675864 N^{5}+2362028 N^{4}-3013280 N^{3} \\
& +2221520 N^{2}-926400 N+177280 \\
P_{8,1}= & 27 N^{5}-54 N^{4}+39 N^{3}+7 N^{2}-36 N+20 \\
P_{8,2}= & 540 N^{6}+765 N^{5}-2364 N^{4}+2098 N^{3}-686 N^{2}-592 N+488 \\
P_{8,3}= & 90 N^{6}+75 N^{5}-198 N^{4}+156 N^{3}-202 N^{2}+216 N-104 \\
P_{8,4}= & 255 N^{4}-78 N^{3}-310 N^{2}+218 N-28 \\
P_{8,5}= & 69 N^{4}-156 N^{3}+52 N^{2}+178 N-164 \\
P_{8,6}= & 1188 N^{6}+3519 N^{5}-2868 N^{4}-5066 N^{3}+9654 N^{2}-8560 N+3000 \\
P_{8,7}= & 87 N^{5}+81 N^{4}-302 N^{3}+396 N^{2}-214 N+60 \\
P_{8,8}= & 99 N^{5}-12 N^{4}-486 N^{3}+706 N^{2}-640 N+168 \\
P_{8,9}= & 21 N^{4}+48 N^{3}-36 N^{2}-2 N-12 \\
P_{8,10}= & 51 N^{4}+138 N^{3}-86 N^{2}-142 N-12 \\
P_{8,11}= & 33 N^{4}+48 N^{3}-164 N^{2}+2 N+156 \\
P_{8,12}= & 33 N^{4}+54 N^{3}-58 N^{2}+134 N-36 \\
P_{8,13}= & 3 N^{3}+9 N^{2}-5 N-14
\end{aligned}
$$


For $p=[4,2]$ the operator is

$$
\begin{aligned}
& S_{[4,2]}^{\mathrm{BPS}}=\frac{1}{6 \sqrt{3 P_{9}}}\left(-\sqrt{2} P_{9,1} \mathcal{O}_{\text {甲四 }}-6 \sqrt{2} P_{9,2} \mathcal{O}_{\text {巴耳, }, 1}-3 \sqrt{2} P_{9,3} \mathcal{O}_{\text {田 }, 2}-4 \sqrt{15} N P_{9,4} \mathcal{O}_{\text {四 }, 1}\right. \\
& -2 \sqrt{3} P_{9,5} \mathcal{O}_{\text {四, } 2}+(N+3)\left[-10 \sqrt{2}(N-1) P_{9,6} \mathcal{O}_{\boxplus}+6 \sqrt{2} P_{9,7}\left\{N \mathcal{O}_{\boxplus, 1}+2 \sqrt{2} \mathcal{O}_{\boxplus, 2}\right\}\right. \\
& -\sqrt{2} P_{9,8}\left\{8 \sqrt{2}(N-1) \mathcal{O}_{\text {甲, }}-3(N+2) \mathcal{O}_{\text {田 }}, 2\right\}+4 \sqrt{15}(N+1)(N-1) N \mathcal{O}_{\text {田,1 }} \\
& \left.\left.-2 \sqrt{3} P_{9,9} \mathcal{O}_{\text {目 }, 2}-\sqrt{2}(N+2)\left\{10 \mathcal{O}_{\text {田 }}+6 \mathcal{O}_{\text {田 }}, 1+P_{9,10} \mathcal{O}_{\text {目 }}\right\}\right]\right)
\end{aligned}
$$

where the normalisation and coefficient polynomials are

$$
\begin{aligned}
P_{9}= & 297 N^{10}+378 N^{8}-1260 N^{7}+390 N^{6}+1080 N^{5}-1256 N^{4}+640 N^{3} \\
& +760 N^{2}-1920 N+1440 \\
P_{9,1}= & 81 N^{5}-129 N^{4}+51 N^{3}+76 N^{2}-130 N+60 \\
P_{9,2}= & 9 N^{5}+3 N^{4}-3 N^{3}-13 N^{2}+13 N-6 \\
P_{9,3}= & \left(9 N^{3}+13 N^{2}-13 N+6\right)\left(3 N^{2}-2\right) \\
P_{9,4}= & 5 N^{3}-2 N^{2}-5 N+3 \\
P_{9,5}= & 29 N^{4}-65 N^{3}+24 N^{2}+70 N-60 \\
P_{9,6}= & 3 N^{2}-3 N+2 \\
P_{9,7}= & N^{2}-2 N+2 \\
P_{9,8}= & 3 N^{2}-2 \\
P_{9,9}= & 11 N^{3}-18 N^{2}-10 N+20 \\
P_{9,10}= & 9 N^{2}-10
\end{aligned}
$$

\section{B.3 Norms of operators with multiplicity}

As explained in section 6.7 , for $\Lambda, p$ with $\mathcal{M}_{\Lambda, p} \geq 1$, the BPS norms of the operators are dependent on the choice of basis for the multiplicity space. In that section, we described a process to extract norm-like functions of $N$ that characterise the multiplicity space and are independent of the choice of basis.

In the $\Lambda=[4,2]$ sector, there are two partitions $p=[2,2,1,1]$ and $[3,2,1]$ with $\mathcal{M}_{\Lambda, p}=2$. For the first of these, we go through the process described in section 6.7 in some detail, while for the second we only give the results.

We begin by renormalising the BPS operators to have norm 1 in the physical inner product as given in (6.72). For $p=[2,2,1,1]$, this replaces $P_{1}$ and $P_{2}$ in the expansions (B.33) and (B.45) with

$$
\begin{aligned}
& \widehat{P}_{1}=3(N+3)(N+2)(N+1)^{2} N^{2}(N-1)(N-2)(N-3) Q_{\text {mult }} Q_{1} \\
& \widehat{P}_{2}=3(N+1) N^{2}(N-1)(N-2)(N-3) Q_{\text {mult }} Q_{2}
\end{aligned}
$$


where $Q_{1}$ and $Q_{2}$ are defined in (B.181) and (B.182) and

$$
\begin{aligned}
Q_{\text {mult }}= & 2145 N^{8}+21570 N^{7}+69156 N^{6}+44856 N^{5}-130747 N^{4}-117106 N^{3}+138802 N^{2} \\
& +53280 N-75456
\end{aligned}
$$

after normalising, the $S_{n}$ inner product matrix can be calculated, and is given by

$$
A_{[2,2,1,1]}=\left(\begin{array}{ll}
\left\langle\widehat{S}_{[2,2,1,1], 1}^{\mathrm{BPS}} \mid \widehat{S}_{[2,2,1,1], 1}^{\mathrm{BPS}}\right\rangle_{S_{n}} & \left\langle\widehat{S}_{[2,2,1,1], 1}^{\mathrm{BPS}} \mid \widehat{S}_{[2,2,1,1], 2}^{\mathrm{BPS}}\right\rangle_{S_{n}} \\
\left\langle\widehat{S}_{[2,2,1,1], 2}^{\mathrm{BPS}} \mid \widehat{S}_{[2,2,1,1], 1}^{\mathrm{BPS}}\right\rangle_{S_{n}} & \left\langle\widehat{S}_{[2,2,1,1], 2}^{\mathrm{BPS}} \mid \widehat{S}_{[2,2,1,1], 2}^{\mathrm{BPS}}\right\rangle_{S_{n}}
\end{array}\right)=\left(\begin{array}{cc}
\frac{A_{1,1}}{\widehat{P}_{1}} & \frac{A_{1,2}}{\sqrt{\widehat{P}_{1} \widehat{P}_{2}}} \\
\frac{A_{1,2}}{\sqrt{\widehat{P}_{1} \widehat{P}_{2}}} & \frac{A_{2,2}}{\widehat{P}_{2}}
\end{array}\right)
$$

where

$$
\begin{aligned}
A_{1,1}= & 1254825 N^{16}+25236900 N^{15}+212913135 N^{14}+949347864 N^{13}+2265287922 N^{12}+2296326096 N^{11} \\
& -483268806 N^{10}-64991400 N^{9}+7717590681 N^{8}+4250132076 N^{7}-14563157385 N^{6} \\
& -5596987632 N^{5}+20300164460 N^{4}+5660498272 N^{3}-5514459136 N^{2}+14594125824 N \\
& +12396386304 \\
A_{1,2}= & \sqrt{5}\left(394875 N^{15}+7400484 N^{14}+57527991 N^{13}+231664914 N^{12}+476892396 N^{11}+249273666 N^{10}\right. \\
& -1301445666 N^{9}-4474130634 N^{8}-7919982621 N^{7}-8401406142 N^{6}-6257132757 N^{5} \\
& \left.-4801800696 N^{4}-1575438250 N^{3}-1395294808 N^{2}-4205573568 N-1295069184\right) \\
A_{2,2}= & 64575225 N^{16}+1221543180 N^{15}+9292923450 N^{14}+34312809600 N^{13}+49747071546 N^{12} \\
& -49520811024 N^{11}-212528733480 N^{10}+81502221096 N^{9}+872883407025 N^{8}+609873915684 N^{7} \\
& -949480261506 N^{6}-778095650280 N^{5}+986491220724 N^{4}+591265527264 N^{3}-532623199736 N^{2} \\
& -150593123520 N+181872634752
\end{aligned}
$$

We now take the trace of $A_{[2,2,1,1]}$, divide by $\mathcal{M}_{\Lambda, p}$, and take the reciprocal. This gives the first $p=[2,2,1,1]$ invariant

$$
\frac{2}{\operatorname{Tr} A_{[2,2,1,1]}}=\frac{2(N+3)(N+2)(N+1)^{2} N^{2}(N-1)(N-2)(N-3) Q_{1} Q_{2}}{D_{1}}
$$

where the denominator is

$$
\begin{aligned}
D_{1}= & 3913650 N^{16}+78795855 N^{15}+656781957 N^{14}+2811679470 N^{13}+5818416030 N^{12}+1501757316 N^{11} \\
& -15672370512 N^{10}-14255947158 N^{9}+42286367112 N^{8}+71992040249 N^{7}-32371301901 N^{6} \\
& -121059621624 N^{5}-22843286488 N^{4}+77152295508 N^{3}+42542435352 N^{2}+5036467584 N \\
& +2255817600
\end{aligned}
$$

We can also consider the trace of $A_{[2,2,1,1]}^{2}$. This leads to the second invariant

$$
\sqrt{\frac{2}{\operatorname{Tr} A_{[2,2,1,1]}^{2}}}=\frac{\sqrt{2}(N+3)(N+2)(N+1)^{2} N^{2}(N-1)(N-2)(N-3) Q_{1} Q_{2}}{\sqrt{D_{2}}}
$$


where the denominator is

$$
\begin{aligned}
D_{2}= & 7658328161250 N^{32}+308379397920750 N^{31}+5678040590961075 N^{30}+62861883407800200 N^{29} \\
& +461553133569402069 N^{28}+2323880655128992368 N^{27}+7893896923770889320 N^{26} \\
& +16200841926037924512 N^{25}+9738474984510581700 N^{24}-43140893567922372492 N^{23} \\
& -100830809456338189482 N^{22}+66300678032545590264 N^{21}+576422366985618028290 N^{20} \\
& +587496624365125252152 N^{19}-1266939757691694906384 N^{18}-3370314414344723267400 N^{17} \\
& -14422779155617085790 N^{16}+8873284172309294711934 N^{15}+9228283693975324117807 N^{14} \\
& -8309143471774592802944 N^{13}-21871661389590847910159 N^{12}-3725069874701998817592 N^{11} \\
& +25451491117140266214976 N^{10}+18757146605106723110568 N^{9}-15395309506022451870416 N^{8} \\
& -22339442519546818907728 N^{7}+3985689055612424950064 N^{6}+16657691069689910952704 N^{5} \\
& +3604888800092578331072 N^{4}-4775351642112978422784 N^{3}-82696688563225374720 N^{2} \\
& +2740871464097166655488 N+1006239182315089379328
\end{aligned}
$$

For $p=[3,2,1]$, the same process produces

$$
\begin{aligned}
\frac{2}{\operatorname{Tr} A_{[3,2,1]}} & =\frac{2(N+2)(N+1) N^{2}(N-1)(N-2) Q_{4} Q_{5}}{E_{1}} \\
\sqrt{\frac{2}{\operatorname{Tr} A_{[3,2,1]}^{2}}} & =\frac{\sqrt{2}(N+2)(N+1) N^{2}(N-1)(N-2) Q_{4} Q_{5}}{\sqrt{E_{2}}}
\end{aligned}
$$

where the denominators are

$$
\begin{aligned}
E_{1}= & 41812200 N^{14}+448198920 N^{13}+1563219648 N^{12}+1093147920 N^{11}-3204936072 N^{10} \\
& -1375066305 N^{9}+4730520504 N^{8}-3314823954 N^{7}+4335640504 N^{6}-6084970 N^{5} \\
& -10209076192 N^{4}+9690911824 N^{3}-2443216896 N^{2}+3777810528 N-538272768
\end{aligned}
$$

$E_{2}=874130034420000 N^{28}+18740182882824000 N^{27}+166345754746996800 N^{26}$

$+753788224097235360 N^{25}+1608498415010610504 N^{24}+181361766700128024 N^{23}$

$-5390210561323512672 N^{22}-4416942361725000252 N^{21}+13381736971853528568 N^{20}$

$+14451638301852715944 N^{19}-26479800963850159935 N^{18}-31944440045187411534 N^{17}$

$+47031196210114852566 N^{16}+73597499966176725312 N^{15}-112834178522277863808 N^{14}$

$-122423268066628273308 N^{13}+309535922049432602720 N^{12}+10889533588200882344 N^{11}$

$-425645164341054775804 N^{10}+196537079346722192144 N^{9}+367648860348492413280 N^{8}$

$-423712842979380230656 N^{7}+67969647225996116864 N^{6}+101859033408413821440 N^{5}$

$-27177241919312392192 N^{4}-38998356711672686592 N^{3}+48696174595572179968 N^{2}$

$-18571148044644937728 N+3609255644969508864$

\section{B.4 Norms of BPS operators}

The physical norms of the BPS operators can be understood as characteristic functions of the pair $\Lambda, p$ and should be reproducible from stringy physics on the other side of the AdS/CFT duality. 
We give the norms for each of the BPS operators in the $\Lambda=[4,2]$ sector. For $p=$ $[2,2,1,1]$ and $[3,2,1]$, we reproduce the invariants derived in the previous subsection in order to compare with other operators.

$$
\begin{aligned}
\left|S_{[2,1,1,1,1]}^{\mathrm{BPS}}\right|^{2} & =\frac{(N+4)(N+3)(N+2)(N+1) N^{2}(N-1)^{2}(N-2)(N-3)(N-4) Q_{1}}{P_{0}} \\
\frac{2}{\operatorname{Tr} A_{[2,2,1,1]}} & =\frac{2(N+3)(N+2)(N+1)^{2} N^{2}(N-1)(N-2)(N-3) Q_{1} Q_{2}}{D_{1}} \\
\sqrt{\frac{2}{\operatorname{Tr} A_{[2,2,1,1]}^{2}}} & =\frac{\sqrt{2}(N+3)(N+2)(N+1)^{2} N^{2}(N-1)(N-2)(N-3) Q_{1} Q_{2}}{\sqrt{D_{2}}} \\
\left|S_{[3,1,1,1]}^{\mathrm{BPS}}\right|^{2} & =\frac{(N+2)(N+1) N(N-1)(N-2)(N-3) Q_{2} Q_{3}}{2 P_{3}} \\
\left|S_{[2,2,2]}^{\mathrm{BPS}}\right|^{2} & =\frac{(N+1) N^{2}(N-1)(N-2) Q_{3} Q_{4}}{P_{4}} \\
\frac{2}{\operatorname{Tr} A_{[3,2,1]}} & =\frac{2(N+2)(N+1) N^{2}(N-1)(N-2) Q_{4} Q_{5}}{E_{1}} \\
\sqrt{\frac{2}{\operatorname{Tr} A_{[3,2,1]}^{2}}} & =\frac{\sqrt{2}(N+2)(N+1) N^{2}(N-1)(N-2) Q_{4} Q_{5}}{\sqrt{E_{2}}} \\
\left|S_{[4,1,1]}^{\mathrm{BPS}}\right|^{2} & =\frac{(N+3)(N+2)(N+1) N(N-1)(N-2) Q_{5} Q_{6}}{P_{7}} \\
\left|S_{[3,3]}^{\mathrm{BPS}}\right|^{2} & =\frac{(N+2)(N+1) N^{2}(N-1) Q_{6} Q_{7}}{P_{8}} \\
\left|S_{[4,2]}^{\mathrm{BPS}}\right|^{2} & =\frac{(N+3)(N+2)(N+1) N^{4}(N-1)\left(3 N^{2}-2\right) Q_{7}}{P_{9}}
\end{aligned}
$$

where the polynomials in the denominator have been defined in previous subsections and the polynomials in the numerator are

$$
\begin{aligned}
Q_{1}= & 195 N^{5}+1149 N^{4}+687 N^{3}-3927 N^{2}-1552 N+4448 \\
Q_{2}= & 10035 N^{8}+94914 N^{7}+264876 N^{6}+17268 N^{5}-819309 N^{4}-487830 N^{3}+780722 N^{2} \\
& +189568 N-432744 \\
Q_{3}= & 18630 N^{8}+160677 N^{7}+371643 N^{6}-204495 N^{5}-1326729 N^{4}-15804 N^{3}+1726178 N^{2} \\
& -442368 N-1298232 \\
Q_{4}= & 8010 N^{7}+56214 N^{6}+79800 N^{5}-132315 N^{4}-158273 N^{3}+296994 N^{2} \\
& +33500 N-171336 \\
Q_{5}= & 2610 N^{7}+12546 N^{6}+3213 N^{5}-25152 N^{4}+20228 N^{3}-5238 N^{2}-8000 N+5160 \\
Q_{6}= & 648 N^{7}+2772 N^{6}+51 N^{5}-5484 N^{4}+5438 N^{3}-2026 N^{2}-2000 N+1720 \\
Q_{7}= & 99 N^{6}+162 N^{5}-324 N^{4}+102 N^{3}+152 N^{2}-260 N+120
\end{aligned}
$$

Comparing these norms with those in sections A.2 and 6.7, we see a general pattern in the numerators. They typically contain a product of linear factors along with (in general) two complicated $Q$ polynomials. These $Q$ polynomials appear in two consecutive norms. 
In (B.158) we saw that the $Q$ polynomials appear in consecutive norms even in the non-physical multiplicity space. This suggests they are an artefact of the orthogonalisation process.

The linear factors are more interesting. Their presence is partially implied by SEPcompatibility, but there are generally more factors than would be sufficient for this purpose. The function $f_{p}$, defined in (2.29), that gives the free field norms, is a product of linear factors, and we can compare this with those found in the numerators of weak coupling BPS norms. In all but two $(p=[2,2,2]$ and $[3,3])$ of the examples we have calculated, the numerators contain $f_{p}$, while some partitions have considerably more factors. It would be interesting to enumerate the linear factors that appear in the numerator for general $\Lambda, p$.

\section{$\mathrm{C} \quad \Lambda=[3,3]$ sector}

The final example we give here is the BPS basis for the $\Lambda=[3,3]$ sector at field content $(3,3)$.

Throughout this section we will work with $\Lambda=[3,3]$ and $M_{\Lambda}=\begin{array}{lll}\frac{1}{1} & 1 & 1 \\ 2 & 2 & 2\end{array}$, so we will suppress this index in operator labels.

For each BPS operator, we will first present it as a sum over the free field basis (2.64) and then as a sum over symmetrised traces and commutator traces, for which we use the covariant bases discussed in section 5.2. The covariant symmetrised trace basis is

$$
\begin{aligned}
t_{[3,2,1]}= & \operatorname{Tr} X^{3} \operatorname{Tr} Y \operatorname{Tr} Y^{2}-2 \operatorname{Tr} X^{2} Y \operatorname{Tr} X Y \operatorname{Tr} Y-\operatorname{Tr} X \operatorname{Tr} X^{2} Y \operatorname{Tr} Y^{2} \\
& +\operatorname{Tr} X^{2} \operatorname{Tr} X Y^{2} \operatorname{Tr} Y+2 \operatorname{Tr} X \operatorname{Tr} X Y \operatorname{Tr} X Y^{2}-\operatorname{Tr} X \operatorname{Tr} X^{2} \operatorname{Tr} Y^{3} \\
t_{[3,1,1,1]}= & \operatorname{Tr} X^{3}(\operatorname{Tr} Y)^{3}-3 \operatorname{Tr} X \operatorname{Tr} X^{2} Y(\operatorname{Tr} Y)^{2}+3(\operatorname{Tr} X)^{2} \operatorname{Tr} X Y^{2} \operatorname{Tr} Y \\
& +(\operatorname{Tr} X)^{3} \operatorname{Tr} Y^{3}
\end{aligned}
$$

and the covariant commutator trace basis is

$$
\begin{aligned}
c_{[6]} & =\operatorname{Tr} X^{2} Y X Y^{2}-\operatorname{Tr} X^{2} Y^{2} X Y=\operatorname{Tr} X^{2} Y[X, Y] Y \\
c_{[5,1]} & =\operatorname{Tr} X^{3} Y^{2} \operatorname{Tr} Y-\operatorname{Tr} X^{2} Y X Y \operatorname{Tr} Y-\operatorname{Tr} X \operatorname{Tr} X^{2} Y^{3}+\operatorname{Tr} X \operatorname{Tr} X Y X Y^{2} \\
& =\operatorname{Tr} X^{2}[X, Y] Y \operatorname{Tr} Y-\operatorname{Tr} X \operatorname{Tr} X[X, Y] Y^{2}
\end{aligned}
$$

For these two bases, the partition label describes the cycle structure of the multi-traces.

The free field operators can be written in terms of symmetrised and commutator traces

$$
\begin{aligned}
O_{\boxminus} & =\frac{\sqrt{3}}{36}\left(3 t_{[3,2,1]}+t_{[3,1,1,1]}+6 c_{[5,1]}\right) \\
O_{\Xi}, \text { even } & =\frac{\sqrt{3}}{18}\left(t_{[3,1,1,1]}-3 c_{[5,1]}\right) \\
O_{\boxplus}, \text { odd } & =-\frac{1}{\sqrt{2}} c_{[6]} \\
O_{\text {目 }} & =\frac{\sqrt{3}}{36}\left(-3 t_{[3,2,1]}+t_{[3,1,1,1]}+6 c_{[5,1]}\right)
\end{aligned}
$$


The odd/even labels for the $R=[3,2,1]$ multiplicity come from the odd/even permutations used to produce the respective traces. All other zero coupling operators are defined uniquely by $\Lambda$ and $R$.

The BPS operators are

$$
\begin{aligned}
& S_{[3,1,1,1]}^{\mathrm{BPS}}=\frac{1}{\sqrt{3 P_{1}}}\left(-N(N-3) O_{\text {目 }}+(N+3)(N-3) O_{\boxplus, \text { even }}+2(N+3) N O_{\text {目 }}\right) \\
& =\frac{1}{12 \sqrt{P_{1}}}\left(-3 N(N+1) t_{[3,2,1]}+\left(N^{2}+3 N-6\right) t_{[3,1,1,1]}+18(N+1) c_{[5,1]}\right) \\
& S_{[3,2,1]}^{\mathrm{BPS}}=\frac{1}{\sqrt{P_{2}}}\left((N-1) O_{\text {四 }}+(N+1) O_{\text {甲 }}, \text { even }-O_{\text {目 }}\right) \\
& =\frac{1}{4 \sqrt{3 P_{2}}}\left(N t_{[3,2,1]}+N t_{[3,1,1,1]}-6 c_{[5,1]}\right)
\end{aligned}
$$

where the normalisation polynomials are

$$
P_{1}=2 N^{4}+6 N^{3}+9 N^{2}+27 \quad P_{2}=2 N^{2}+3
$$

The norms of the BPS operators are

$$
\begin{aligned}
\left|S_{[3,1,1,1]}^{\mathrm{BPS}}\right|^{2} & =\frac{(N+3)(N+2)(N+1) N^{2}(N-1)(N-2)(N-3) Q}{P_{1}} \\
\left|S_{[3,2,1]}^{\mathrm{BPS}}\right|^{2} & =\frac{(N+2)(N+1) N^{2}(N-1)(N-2) Q}{P_{2}}
\end{aligned}
$$

where

$$
Q=2 N^{2}+3 N-3
$$

Open Access. This article is distributed under the terms of the Creative Commons Attribution License (CC-BY 4.0), which permits any use, distribution and reproduction in any medium, provided the original author(s) and source are credited.

\section{References}

[1] J.M. Maldacena, The large $N$ limit of superconformal field theories and supergravity, Adv. Theor. Math. Phys. 2 (1998) 231 [Int. J. Theor. Phys. 38 (1999) 1113] [hep-th/9711200] [INSPIRE].

[2] S.S. Gubser, I.R. Klebanov and A.M. Polyakov, Gauge theory correlators from noncritical string theory, Phys. Lett. B 428 (1998) 105 [hep-th/9802109] [INSPIRE].

[3] E. Witten, Anti-de Sitter space and holography, Adv. Theor. Math. Phys. 2 (1998) 253 [hep-th/9802150] [INSPIRE].

[4] J. McGreevy, L. Susskind and N. Toumbas, Invasion of the giant gravitons from Anti-de Sitter space, JHEP 06 (2000) 008 [hep-th/0003075] [INSPIRE].

[5] M.T. Grisaru, R.C. Myers and O. Tafjord, SUSY and goliath, JHEP 08 (2000) 040 [hep-th/0008015] [INSPIRE].

[6] A. Hashimoto, S. Hirano and N. Itzhaki, Large branes in AdS and their field theory dual, JHEP 08 (2000) 051 [hep-th/0008016] [INSPIRE]. 
[7] H. Lin, O. Lunin and J.M. Maldacena, Bubbling AdS space and 1/2 BPS geometries, JHEP 10 (2004) 025 [hep-th/0409174] [InSPIRE].

[8] V. Balasubramanian, M. Berkooz, A. Naqvi and M.J. Strassler, Giant gravitons in conformal field theory, JHEP 04 (2002) 034 [hep-th/0107119] [INSPIRE].

[9] S. Corley, A. Jevicki and S. Ramgoolam, Exact correlators of giant gravitons from dual $N=4$ SYM theory, Adv. Theor. Math. Phys. 5 (2002) 809 [hep-th/0111222] [INSPIRE].

[10] J.M. Maldacena and A. Strominger, $A d S_{3}$ black holes and a stringy exclusion principle, JHEP 12 (1998) 005 [hep-th/9804085] [INSPIRE].

[11] A. Bissi, C. Kristjansen, D. Young and K. Zoubos, Holographic three-point functions of giant gravitons, JHEP 06 (2011) 085 [arXiv:1103.4079] [INSPIRE].

[12] P. Caputa, R. de Mello Koch and K. Zoubos, Extremal versus Non-Extremal Correlators with Giant Gravitons, JHEP 08 (2012) 143 [arXiv: 1204.4172] [INSPIRE].

[13] H. Lin, Giant gravitons and correlators, JHEP 12 (2012) 011 [arXiv:1209.6624] [INSPIRE].

[14] C. Kristjansen, S. Mori and D. Young, On the Regularization of Extremal Three-point Functions Involving Giant Gravitons, Phys. Lett. B 750 (2015) 379 [arXiv:1507.03965] [INSPIRE].

[15] A. Mikhailov, Giant gravitons from holomorphic surfaces, JHEP 11 (2000) 027 [hep-th/0010206] [INSPIRE].

[16] C.E. Beasley, BPS branes from baryons, JHEP 11 (2002) 015 [hep-th/0207125] [INSPIRE].

[17] I. Biswas, D. Gaiotto, S. Lahiri and S. Minwalla, Supersymmetric states of $N=4$ Yang-Mills from giant gravitons, JHEP 12 (2007) 006 [hep-th/0606087] [INSPIRE].

[18] G. Mandal and N.V. Suryanarayana, Counting 1/8-BPS dual-giants, JHEP 03 (2007) 031 [hep-th/0606088] [INSPIRE].

[19] D. Berenstein, Large N BPS states and emergent quantum gravity, JHEP 01 (2006) 125 [hep-th/0507203] [INSPIRE].

[20] J. Kinney, J.M. Maldacena, S. Minwalla and S. Raju, An index for 4 dimensional super conformal theories, Commun. Math. Phys. 275 (2007) 209 [hep-th/0510251] [INSPIRE].

[21] A.V. Ryzhov, Quarter BPS operators in N=4 SYM, JHEP 11 (2001) 046 [hep-th/0109064] [INSPIRE].

[22] E. D'Hoker, P. Heslop, P. Howe and A.V. Ryzhov, Systematics of quarter BPS operators in $N=4$ SYM, JHEP 04 (2003) 038 [hep-th/0301104] [INSPIRE].

[23] E. D'Hoker and A.V. Ryzhov, Three point functions of quarter BPS operators in $N=4$ SYM, JHEP 02 (2002) 047 [hep-th/0109065] [INSPIRE].

[24] A.V. Ryzhov, Operators in the $d=4, N=4 S Y M$ and the AdS/CFT correspondence, other thesis, (2003) [hep-th/0307169] [INSPIRE].

[25] T.W. Brown, P.J. Heslop and S. Ramgoolam, Diagonal multi-matrix correlators and BPS operators in $N=4$ SYM, JHEP 02 (2008) 030 [arXiv:0711.0176] [INSPIRE].

[26] T.W. Brown, P.J. Heslop and S. Ramgoolam, Diagonal free field matrix correlators, global symmetries and giant gravitons, JHEP 04 (2009) 089 [arXiv:0806.1911] [INSPIRE].

[27] Y. Kimura and S. Ramgoolam, Branes, anti-branes and brauer algebras in gauge-gravity duality, JHEP 11 (2007) 078 [arXiv:0709.2158] [INSPIRE]. 
[28] R. Bhattacharyya, S. Collins and R. de Mello Koch, Exact Multi-Matrix Correlators, JHEP 03 (2008) 044 [arXiv: 0801.2061] [inSPIRE].

[29] R. Bhattacharyya, R. de Mello Koch and M. Stephanou, Exact Multi-Restricted Schur Polynomial Correlators, JHEP 06 (2008) 101 [arXiv:0805.3025] [INSPIRE].

[30] Y. Kimura and S. Ramgoolam, Enhanced symmetries of gauge theory and resolving the spectrum of local operators, Phys. Rev. D 78 (2008) 126003 [arXiv:0807.3696] [InSPIRE].

[31] T.W. Brown, Cut-and-join operators and $N=4$ super Yang-Mills, JHEP 05 (2010) 058 [arXiv: 1002.2099] [INSPIRE].

[32] J. Pasukonis and S. Ramgoolam, From counting to construction of BPS states in $N=4$ SYM, JHEP 02 (2011) 078 [arXiv: 1010.1683] [INSPIRE].

[33] J. Pasukonis and S. Ramgoolam, Quantum states to brane geometries via fuzzy moduli spaces of giant gravitons, JHEP 04 (2012) 077 [arXiv:1201.5588] [INSPIRE].

[34] Y. Kimura, Quarter BPS classified by Brauer algebra, JHEP 05 (2010) 103 [arXiv: 1002.2424] [INSPIRE].

[35] F. Vaccarino, Linear representations, symmetric products and the commuting scheme, J. Algebra 317 (2007) 634.

[36] M. Domokos Vector Invariants of a Class of Pseudo-Reflection Groups and Multisymmetric Syzygies, arXiv:0706.2154.

[37] C. Procesi, Invariants of commuting matrices, arXiv:1501.05190.

[38] I.G. Macdonald Symmetric functions and Hall polynomials, Oxford Mathematical Monographs. Oxford University Press (1995).

[39] B.U. Eden, P.S. Howe, E. Sokatchev and P.C. West, Extremal and next-to-extremal $n$ point correlators in four-dimensional SCFT, Phys. Lett. B 494 (2000) 141 [hep-th/0004102] [INSPIRE].

[40] F.A. Dolan and H. Osborn, On short and semi-short representations for four-dimensional superconformal symmetry, Annals Phys. 307 (2003) 41 [hep-th/0209056] [INSPIRE].

[41] S. Collins, Restricted Schur Polynomials and Finite N Counting, Phys. Rev. D 79 (2009) 026002 [arXiv: 0810.4217] [INSPIRE].

[42] M. Baggio, J. de Boer and K. Papadodimas, A non-renormalization theorem for chiral primary 3-point functions, JHEP 07 (2012) 137 [arXiv:1203.1036] [INSPIRE].

[43] N. Beisert, C. Kristjansen, J. Plefka, G.W. Semenoff and M. Staudacher, BMN correlators and operator mixing in $N=4$ superYang-Mills theory, Nucl. Phys. B 650 (2003) 125 [hep-th/0208178] [INSPIRE].

[44] N. Beisert, C. Kristjansen and M. Staudacher, The dilatation operator of conformal $N=4$ superYang-Mills theory, Nucl. Phys. B 664 (2003) 131 [hep-th/0303060] [INSPIRE].

[45] V. Balasubramanian, B. Czech, K. Larjo and J. Simon, Integrability versus information loss: A simple example, JHEP 11 (2006) 001 [hep-th/0602263] [INSPIRE].

[46] K. Skenderis and M. Taylor, Anatomy of bubbling solutions, JHEP 09 (2007) 019 [arXiv: 0706. 0216] [INSPIRE].

[47] D. Berenstein, A toy model for the AdS/CFT correspondence, JHEP 07 (2004) 018 [hep-th/0403110] [INSPIRE]. 
[48] Y. Takayama and A. Tsuchiya, Complex matrix model and fermion phase space for bubbling AdS geometries, JHEP 10 (2005) 004 [hep-th/0507070] [INSPIRE].

[49] A. Donos, A description of $1 / 4$ BPS configurations in minimal type IIB SUGRA, Phys. Rev. $D 75$ (2007) 025010 [hep-th/0606199] [INSPIRE].

[50] B. Chen et al., Bubbling AdS and droplet descriptions of BPS geometries in IIB supergravity, JHEP 10 (2007) 003 [arXiv: 0704.2233] [INSPIRE].

[51] O. Lunin, Brane webs and 1/4-BPS geometries, JHEP 09 (2008) 028 [arXiv:0802.0735] [INSPIRE].

[52] H. Bruus and K. Flensberg, Many-body quantum theory in condensed matter physics, online textbook https://cds.cern.ch/record/1186248/files/0198566336_TOC.pdf.

[53] A. Jevicki and B. Sakita, The Quantum Collective Field Method and Its Application to the Planar Limit, Nucl. Phys. B 165 (1980) 511 [inSPIRE].

[54] S.R. Das and A. Jevicki, String Field Theory and Physical Interpretation of D $=1$ Strings, Mod. Phys. Lett. A 5 (1990) 1639 [InSPIRE].

[55] H. Awata, Y. Matsuo, S. Odake and J. Shiraishi, Collective field theory, Calogero-Sutherland model and generalized matrix models, Phys. Lett. B 347 (1995) 49 [hep-th/9411053] [INSPIRE].

[56] F. Vaccarino, The ring of multisymmetric functions, Annales Inst. Fourier 55 (2005) 717.

[57] R.P. Stanley, Enumerative Combinatorics: Volume 1, second edition, Cambridge studies in Advanced Mathematics, (2012).

[58] R.C. King, Young Tableaux, Schur Functions and SU(2) Plethysms, J. Phys. A 18 (1985) 2429.

[59] R. Goodman and N.R. Wallach, Symmetry, Representations, and Invariants, Graduate Texts in Mathematics, Springer, (2009).

[60] D. Berenstein, Negative specific heat from non-planar interactions and small black holes in AdS/CFT, JHEP 10 (2019) 001 [arXiv:1810.07267] [INSPIRE].

[61] D.E. Berenstein, J.M. Maldacena and H.S. Nastase, Strings in flat space and pp waves from $N=4$ superYang-Mills, JHEP 04 (2002) 013 [hep-th/0202021] [INSPIRE].

[62] R. de Mello Koch, M. Dessein, D. Giataganas and C. Mathwin, Giant Graviton Oscillators, JHEP 10 (2011) 009 [arXiv:1108.2761] [INSPIRE].

[63] R. de Mello Koch and S. Ramgoolam, A double coset ansatz for integrability in AdS/CFT, JHEP 06 (2012) 083 [arXiv: 1204.2153] [INSPIRE].

[64] R. de Mello Koch and L. Nkumane, From Gauss Graphs to Giants, JHEP 02 (2018) 005 [arXiv: 1710.09063] [INSPIRE].

[65] P. Mattioli and S. Ramgoolam, Permutation Centralizer Algebras and Multi-Matrix Invariants, Phys. Rev. D 93 (2016) 065040 [arXiv:1601.06086] [INSPIRE].

[66] R. de Mello Koch, M. Kim and H.J.R. Zyl, Integrable Subsectors from Holography, JHEP 05 (2018) 198 [arXiv: 1802.01367] [INSPIRE].

[67] O. Aharony, Y.E. Antebi, M. Berkooz and R. Fishman, 'Holey sheets': Pfaffians and subdeterminants as D-brane operators in large $N$ gauge theories, JHEP 12 (2002) 069 [hep-th/0211152] [INSPIRE]. 
[68] P. Caputa, R. de Mello Koch and P. Diaz, A basis for large operators in $N=4 S Y M$ with orthogonal gauge group, JHEP 03 (2013) 041 [arXiv:1301.1560] [INSPIRE].

[69] P. Caputa, R. de Mello Koch and P. Diaz, Operators, Correlators and Free Fermions for $\mathrm{SO}(N)$ and $S p(N)$, JHEP 06 (2013) 018 [arXiv:1303.7252] [INSPIRE].

[70] G. Kemp, $\mathrm{SO}(N)$ restricted Schur polynomials, J. Math. Phys. 56 (2015) 022302 [arXiv: 1405.7017] [INSPIRE].

[71] G. Kemp, Restricted Schurs and correlators for $\mathrm{SO}(N)$ and $\mathrm{Sp}(N)$, JHEP 08 (2014) 137 [arXiv: 1406.3854] [INSPIRE].

[72] Y. Kimura, S. Ramgoolam and R. Suzuki, Flavour singlets in gauge theory as Permutations, JHEP 12 (2016) 142 [arXiv:1608.03188] [INSPIRE].

[73] C. Lewis-Brown and S. Ramgoolam, BPS operators in $\mathcal{N}=4 \mathrm{SO}(N)$ super Yang-Mills theory: plethysms, dominoes and words, JHEP 11 (2018) 035 [arXiv: 1804.11090] [INSPIRE].

[74] C. Lewis-Brown, Multi-symmetric functions, Source code, (2020), www.github.com/ChrisL-B/Multi-symmetric-Functions. 